\title{
The molecular anatomy of synaptic vesicle recycling at the hair cell ribbon synapse
}

\author{
Dissertation \\ For the award of the degree \\ "Doctor rerum naturalium" \\ of the Georg-August-Universität Göttingen \\ within the doctoral program Molecular Physiology of the Brain \\ of the Georg-August University School of Science (GAUSS) \\ submitted by \\ Katharina Natalia Richter \\ from Berlin, Germany
}

Göttingen 2019 



\section{Members of the Thesis Advisory Committee}

Prof. Dr. Silvio O. Rizzoli

Department of Neuro- and Sensory Physiology, University of Göttingen Medical Center, Göttingen, Germany

Prof. Dr. Tomas Pieler

Department of Developmental Biochemistry, University of Göttingen Medical Center, Göttingen, Germany

Prof Dr. Carolin Wichmann

Molecular Architecture of Synapses Group Center for Biostructural Imaging of Neurodegeneration (BIN), Institute of Auditory Neuroscience and Inner Ear Lab, University of Göttingen Medical Center, Göttingen, Germany

\section{Members of the Examination Board}

Referee: Prof. Dr. Silvio O. Rizzoli

Department of Neuro- and Sensory Physiology, University of Göttingen Medical Center, Göttingen, Germany

$2^{\text {nd }}$ Referee: Prof. Dr. Tomas Pieler Department of Developmental Biochemistry, University of Göttingen Medical Center, Göttingen, Germany

Prof Dr. Carolin Wichmann

Molecular Architecture of Synapses Group Center for Biostructural Imaging of Neurodegeneration (BIN), Institute of Auditory Neuroscience and Inner Ear Lab, University of Göttingen Medical Center, Göttingen, Germany

\section{Further members of the Examination Board}

Prof. Dr. Nils Brose

Department of Molecular Neurobiology, Max Planck Institute of Experimental Medicine, Göttingen, Germany

Prof. Dr. Ralf Heinrich

Department of Cellular Neurobiology, Schwann-Schleiden-Forschungszentrum, Göttingen, Germany

Prof. Dr. Michael Thumm

Department of Cellular Biochemistry, University of Göttingen Medical Center, Göttingen, Germany

Date of oral examination: 15.08.2019 



\section{Affidativ}

I hereby declare that I prepared this dissertation independently, and with no other sources and aids than quoted.

Katharina Natalia Richter 



\section{Table of Contents}

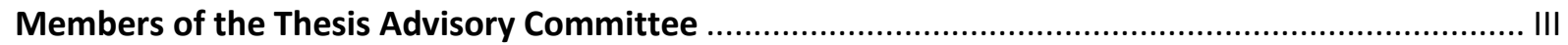

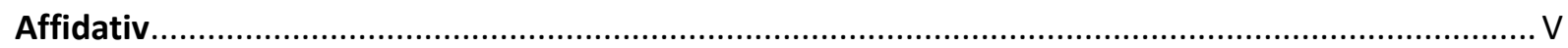

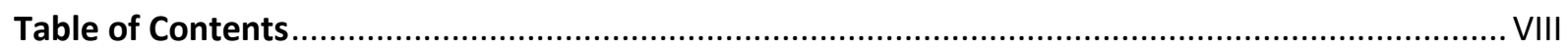

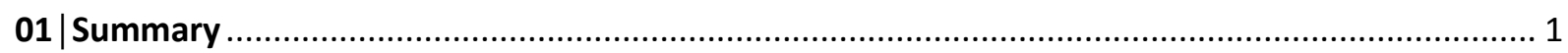

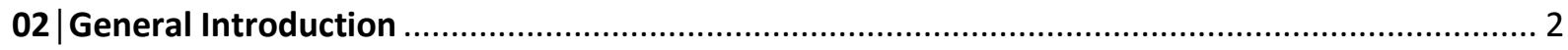

2.1 Structure and function of the auditory system and the inner hair cell ................................... 2

2.2 Structure and developmental changes of the inner hair cell ribbon synapse .......................... 4

2.3 The process of synaptic transmission at the IHC ribbon synapse and its molecular

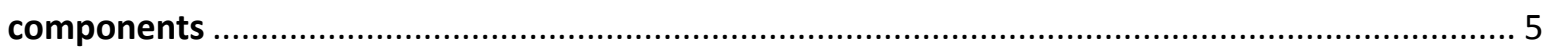

2.4. The need for improvements in existing techniques for the investigation of synaptic proteins

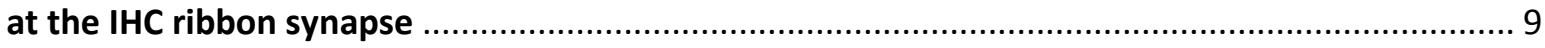

2.5 Improvement of the fixation method for super resolution imaging studies ......................... 10

2.6 CosiQuant: an imaging-based technique for the estimation of protein copy numbers .......... 13

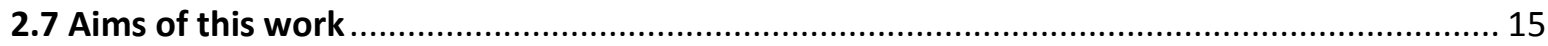

03 | Glyoxal as an alternative fixative to formaldehyde in immunostaining and super-resolution

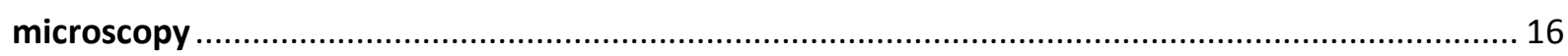

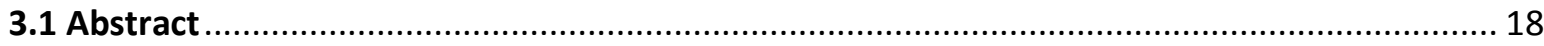

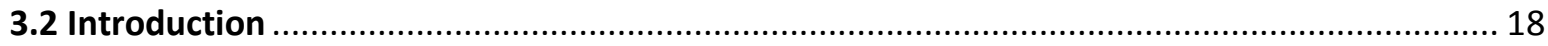

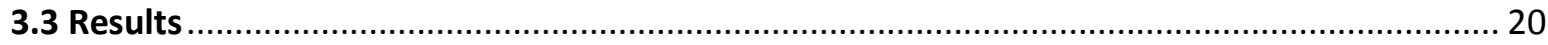

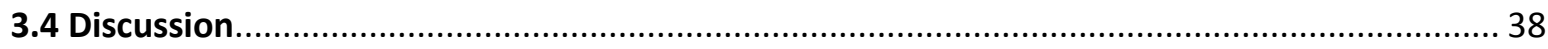

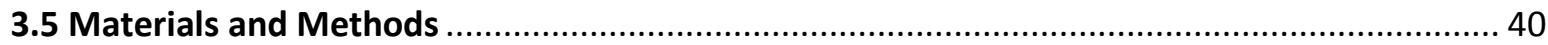

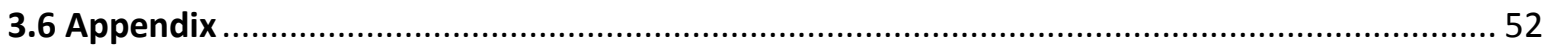

04 |Comparative synaptosome imaging: a semi-quantitative method to obtain ............................... 84

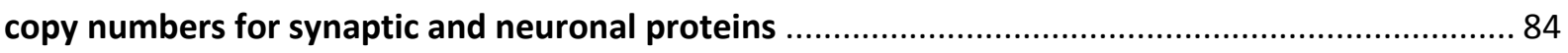

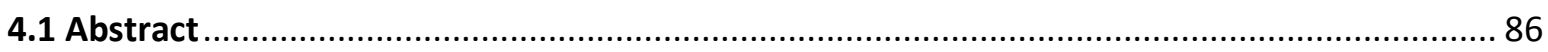

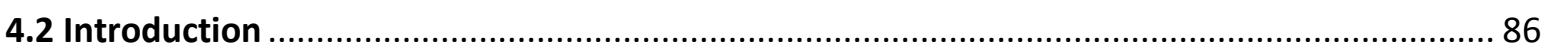

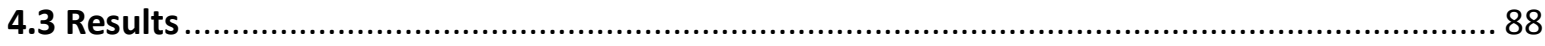

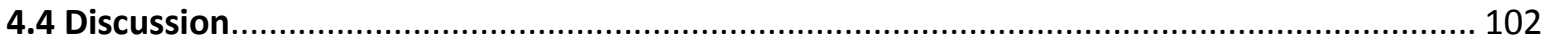

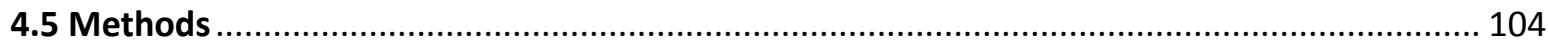

$05 \mid$ The molecular Anatomy of synaptic vesicle recycling at the inner hair cell ribbon synapse .... 109

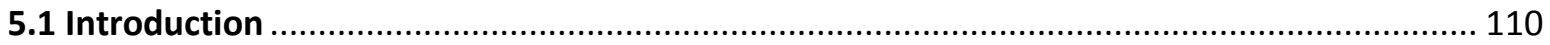

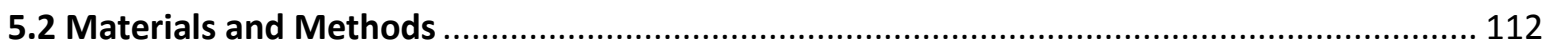

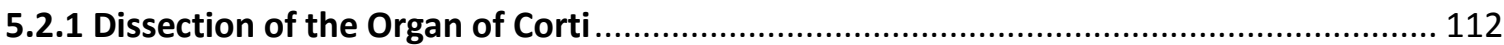


5.2.4 Imaging.

5.2.5 Analysis

5.3 Results

5.3.1 Antibody screening for the immunostaining of synaptic proteins in IHCs

5.3.2 Super-resolution imaging of synaptic proteins for the precise localization within the ribbon synapse

5.3.3 Protein copy number estimation using a comparative imaging approach (CosiQuant) 128

5.3.4 Incorporation of protein copy numbers and spatial organization into a 3-dimensional model

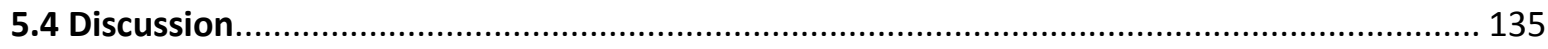

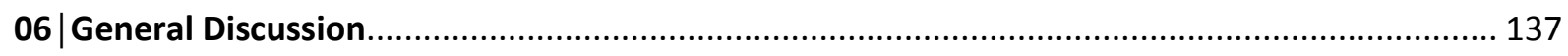

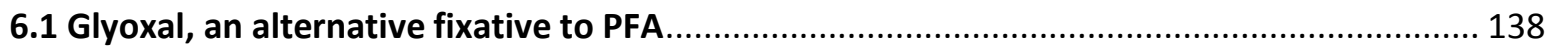

6.2 CosiQuant, a novel method to estimate protein copy numbers ..................................... 143

6.3 Investigation of proteins involved in the synaptic vesicle recycling process at the IHC ribbon

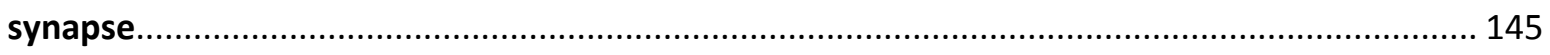

6.4. Relating protein copy numbers to synaptic vesicle release kinetics .............................. 151

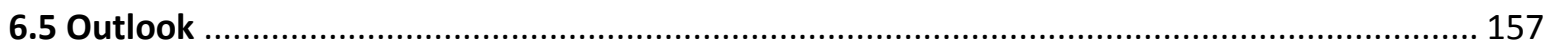

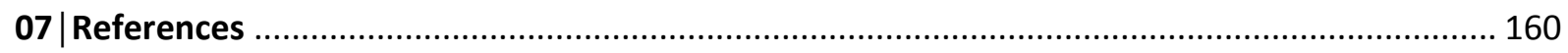

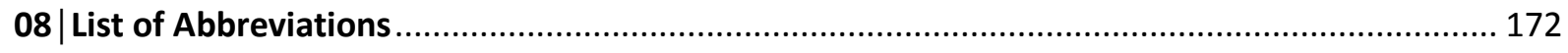

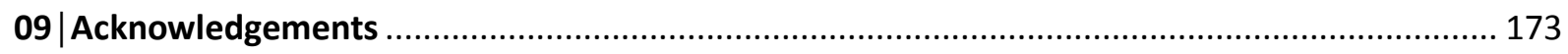

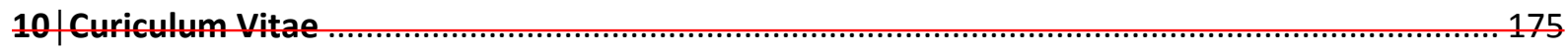




\section{$01 \mid$ Summary}

The inner hair cell (IHC) ribbon synapse of the auditory system is a highly specialized synapse, adapted to very fast transmission rates and maintenance of synaptic vesicle release over long periods of time without showing substantial fatigue. These properties make the IHC ribbon synapse an interesting target to study synaptic vesicle exo- and endocytosis, since there is a need for very efficient synaptic vesicle recycling, in order to ensure vesicle replenishment during prolonged exocytosis, with high release rates. Current knowledge about the function of these synapses mainly stems from electrophysiological measurements, focusing on release kinetics, $\mathrm{Ca}^{2+}$ channel distribution and vesicle pool sizes. However, a detailed picture of the molecular organization of the IHC ribbon synapse, and especially of the proteins involved in the synaptic vesicle recycling process, is still missing. This is due to major difficulties in studying synaptic proteins in the IHC using fluorescence imaging techniques, since immunostaining protocols using commercially-available antibodies often provide insufficient staining quality for IHCs. A better understanding of how the IHC ribbon synapse is organized would facilitate research on functional processes by relating its structure to its function. Therefore, in this work I set out to improve the methods for the investigation of IHC ribbon synapses by immunofluorescence microscopy. I have established glyoxal as an alternative fixative to PFA. Furthermore, I have developed a method (CosiQuant) to estimate protein copy numbers using a comparative imaging approach. CosiQuant is based on the comparison of immunostaining signals between a sample of interest and biochemically-characterized synaptosomes with known protein copy numbers. This method is particularly useful for the investigation of protein amounts in samples that are difficult to analyze with common biochemical techniques, like mass spectrometry, due to problems in sample purification. Finally, I was able to implement both methods to determine the precise localization and estimate the copy numbers of proteins involved in the synaptic vesicle recycling process in IHC ribbon synapses. Glyoxal fixation improved the preservation of a variety of different targets, and the quality of the subsequent immunostainings. This enabled me to image proteins that have been difficult to visualize in the IHC ribbon synapse in the past. Furthermore, using CosiQuant, I could provide first estimates for the copy number of proteins involved in vesicle recycling at the ribbon synapse of IHCs. Combining these data, I was able to generate a preliminary model of the IHC ribbon synapse, containing information about the spatial organization and the abundancy of 19 synaptic proteins, which might be involved in the synaptic vesicle recycling process. Based on this model, I was able to draw assumptions about the functional importance of the investigated proteins. The protein copy number estimates suggested the proteins that might be rate-limiting in the synaptic vesicle recycling process, while the precise protein localization provided information about where exactly exo- and endocytosis take place. Future studies will provide additional information about synaptic proteins, and will thereby increase the accuracy of the model. 


\section{General Introduction}

The first investigations of synapses date back to the late $19^{\text {th }} /$ early $20^{\text {th }}$ century, when the theory of nerve endings was postulated and the term 'synapse' was introduced by Ramon $Y$ Cajal and Charles Scott Sherrington, respectively. As the synapse represents the fundamental basis of neuronal and brain activity, the research interest in synapses and the process of synaptic transmission has grown over the centuries and investigations of functional and structural aspects are still ongoing. The fact that many neurological diseases are connected to misfunctions in synapses makes this topic highly important even in modern research. Probably the most intensly studied synapse is the chemical synapse of the hippocampal neuron. Synaptic transmission in those synapses involves the exocytosis of synaptic vesicles, which releases neurotransmitter into the synaptic cleft where it can be bound by receptors in the postsynaptic membrane. Components of the synaptice vesicle (proteins and membrane lipids), which have fused with the presynaptic plasma membrane are subsequently endocytosed to form new vesicles, a process also referred to as synaptic vesicle recycling (for a detailed review see Südhof, 2004; Haucke et al, 2011; Rizzoli, 2014). The details of this process (i.e. individual steps the synaptic vesicle is going through and the identity of the proteins involved in those steps) are well known for the conventional synapse of the hippocampal neuron. However, this is not the case for all types of synapses. In a very special form of the chemical synapse for example, the ribbon synapse of the auditory system, the details of synaptic vesicle recycling are still largely unknown or at least heavily debated. The overall principle of synaptic vesicle exocytosis and subsequent endocytosis are the same as in the conventional synapse, but due to its specialized role in the transmission of sound, the auditory ribbon synapse has developed distinct differences in order to adapt to this role (Safieddine et al, 2012; Wichmann \& Moser, 2015; Pangršič \& Vogl, 2018).

In the following subsections I would like to briefly introduce the auditory system with its sensory hair cells and the specialized ribbon synapse. Further, I will give an overview of the current knowledge about the process of synaptic vesicle recycling in these synapses and describe improvements I have established to overcome limitations of investigating this process in ribbon synapses.

\subsection{Structure and function of the auditory system and the inner hair cell}

The mammalian auditory system can be divided into the outer ear, the middle ear and the inner ear, which contains the vestibular organ (responsible for balance and orientation) and the cochlea. The cochlea consists of multiple turns of 3 ducts (scala vestibuli, scala media and scala tympani), of which one (the scala media) is harboring the Organ of Corti (Figure $1 \mathrm{~A})$. From the base to the apex of these turns, perception of high to low sound frequencies are represented, respectively (tonotopic map (Rubel, 1984; Mann \& Kelley, 2011)). The Organ of Corti is the place where the mechanical stimulus of a soundwave (caught by the outer ear and travelling through the middle ear) is transduced into an electrochemical signal by the sensory cells of the organ, the hair cells. There are two types of sensory cells in the 
Organ of Corti, the inner hair cells (IHCs) and the outer hair cells (OHCs). The IHCs are responsible for the mechanoelectrical transduction (MET) of the sound signal and the transmission of this signal to afferent neurons projecting to the brain, whereas OHCs function as amplifiers through electromechanical feedback mechanisms (Cooper \& Guinan, 2006; detailed review in Fettiplace \& Hackney, 2006; Fettiplace, 2017). One row of IHCs and three rows of OHCs are surrounded by supporting cells in the Organ or Corti (Figure 1B). The so called hair bundles of both cell types, which consist of multiple rows of stereocilia at the apical cell pole (Furness \& Hackney, 2001), are in contact with the tectorial membrane (Figure 1B). The pressure wave of a sound stimulus is converted at the middle ear into vibrations of the basilar membrane, on which sits the Organ of Corti. This vibration deflects the hair bundles of the inner and outer hair cells against the tectorial membrane and results in the opening of cation channels (mechanoeclectrical transduction channels), thus depolarizing the cells. This stimulation is driven by the electrochemical gradient between the hair cells and the endolymph of the scala media (high $\mathrm{K}^{+}$concentration (Wangemann, 2006)), which surrounds the stereocilia. In OHCs this electrical stimulation results in motile activity, which in turn amplifies the vibration of the basilar membrane and hair bundle deflection of IHCs (Cooper \& Guinan, 2006). In IHCs, depolarization results in the formation of a graded receptor potential, which travels to the base of the cell, where specialized synapses are formed with type I ganglion neurons (Fuchs \& Glowatzki, 2015), the IHC ribbon synapses (Figure 1C). Here, glutamate is exocytosed in a $\mathrm{Ca}^{2+}$-dependent manner, mediated by the voltage-gated $\mathrm{Ca}^{2+}$ channels close to the synapses (Moser et al, 2006). This is driven by the high $\mathrm{Ca}^{2+}$ concentration in the perilymph of the scala tympani, surrounding the base of the IHC (Wangemann, 2006). At the ribbon synapse, the receptor potential of the IHC is converted to frequency modulated action potentials in the afferent auditory neuron with very high temporal precision. IHC ribbon synapses are innervated by afferent neurons in a ratio of 1:1 (Liberman, 1980) and each IHC forms $6-20$ synapses, depending on the tonotopic position along the cochlea turns (Meyer et al, 2009). OHCs on the other hand are innervated by type II ganglion neurons and receive cholinergic input from medial olivochochlear fibers. In addition, lateral olivocochlear fibers synapse with dendrites of afferent neurons (Eybalin, 1993; Guinan, 2010) 
A

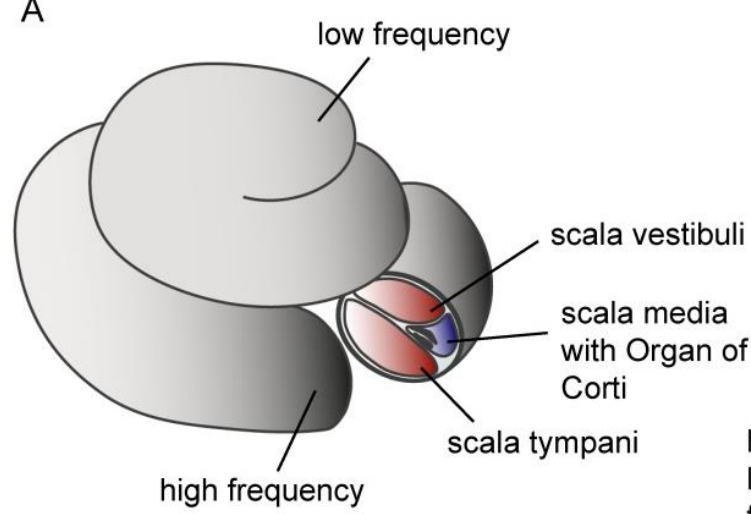

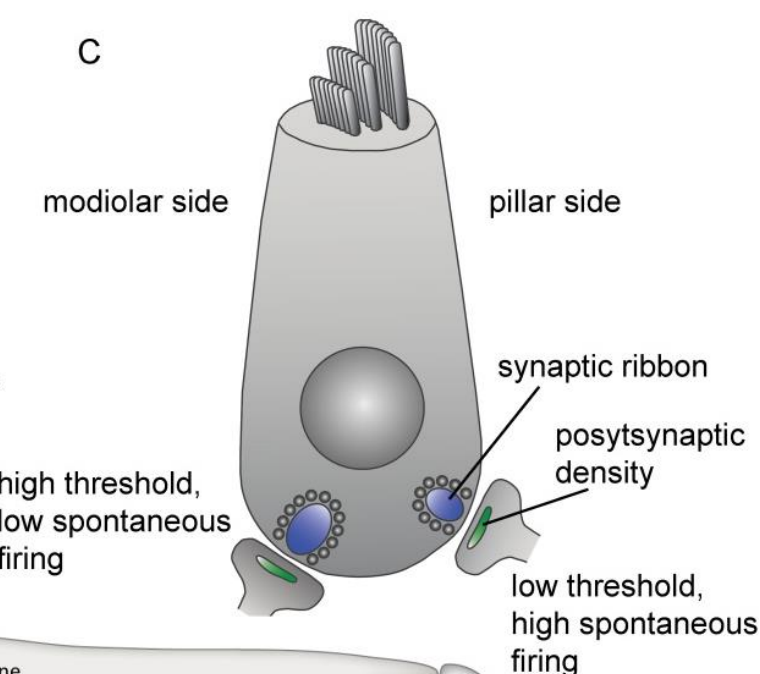
firing

B

B Hair bundle

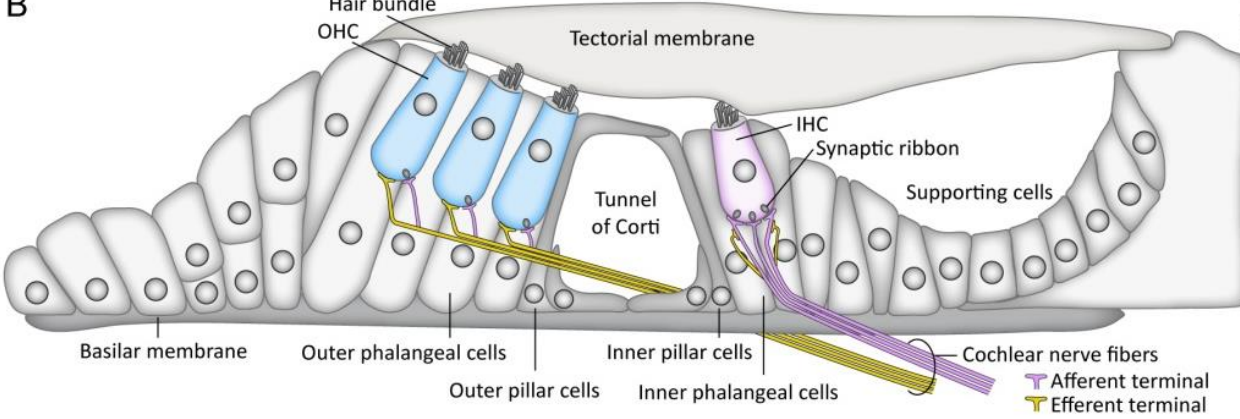

Figure 1 Schematic representation of the cochlea, Organ of Corti and inner hair cell.

A) The cochlea of the inner ear consists of the three ducts scala vestibuli, scala tympani and scala media, which are organized in multiple turns. Along these turns the perception of low to high frequency stimuli is represented from apex to base (tonotopy). The scala media contains the Organ of Corti. B) The Organ of Corti contains three rows of outer hair cells (blue) and one row of inner hair cells (pink), surrounded by supporting cells. The hair bundles of IHCs and OHCs are in contact with the tectorial membrane, which leads to deflection of the hair bundles upon stimulation, which is mediated by vibrations of the basilar membrane. OHCs are innervated by afferent and efferent neurons, whereas IHCs are only in direct contact with afferent ganglion neurons. Efferent innervation can be found in close vicinity onto afferent neurons. Reprinted from the PhD Thesis of Natalia Hasel Revelo Nuncira, published 2015 in the library of the Georg-August University of Göttingen (http://hdl.handle.net/11858/00-1735-0000-0022-5FDB-C) under the creative commons license (https://creativecommons.org/licenses/by-nc-nd/3.0/). C) IHCs form ribbon synapses with type I spiral ganglion neurons. The size of the ribbon (blue) varies between synapses on the modiolar side of the IHC and the pillar side. Ribbons of the modiolar side are larger and form synapses with ganglion neurons exhibiting high firing threshold and low spontaneous firing patterns. Ribbons on the pillar side are smaller and synapse to afferent neurons with lower thresholds and higher spontaneous firing rates.

\subsection{Structure and developmental changes of the inner hair cell ribbon synapse}

The transmission of sound stimuli at the IHC ribbon synapse has to work with a very high temporal precision in order to accurately code stimulus traits. This is especially important e.g. for the recognition of speech cues or the localization of sound sources, which relies on the discrimination of temporal differences of a stimulus arriving at both ears. At the same time, transmission at the IHC synapse needs to be sustained over a long time, as a response to ongoing stimulation lasting up to seconds (Moser \& Beutner, 2000; Schnee et al, 2011). This in turn calls for synapses, which are specialized in a very efficient vesicle recycling 
mechanism, in order to maintain high transmission rates without fatigue, due to depletion of synaptic vesicles. This makes the investigation of the $\mathrm{IHC}$ ribbon synapse so fascinating and gave rise to many studies concerning the structure of the IHC ribbon synapse, in order to relate structure to function (reviewed in Safieddine et al, 2012; Wichmann \& Moser, 2015; Lenzi \& von Gersdorff, 2001; Rutherford \& Pangršič, 2012).

From these past decades of research, a lot has been revealed about the ribbon synapse. The name refers to an electron dense structure at the active zone of these synapses, the ribbon (Smith \& Sjöstrand, 1961; Figure 1C). It consists mainly of the protein ribeye and retains synaptic vesicles close to the active zone (Schmitz et al, 2000; Kantardzhieva et al, 2012; Becker et al, 2018). In adult mammals, the ribbon of IHCs is a protein complex about $200 \mathrm{~nm}$ (Matthews \& Fuchs, 2010) in size with an oval shape. Although size and shape do vary, depending on the location of the hair cell along the cochlea as well as the location of the ribbon within the hair cell (Figure 1C). Ribbons localized on the modiolar side of the IHC are usually larger than ribbons sitting on the pillar side of the hair cell (Liberman et al, 2011). Afferent innervation does also differ in this context. The modiolar side of the IHC builds synapses with spiral ganglion neurons exhibiting low spontaneous firing rates and high firing thresholds, whereas the pillar side forms synapses with ganglion neurons showing low firing thresholds and thus high spontaneous firing rates (Liberman, 1980; Figure 1C).

Heterogeneity in ribbon size, shape and innervation is also found in different developmental stages of the IHC. Prior to the onset of hearing, which is around postnatal day 12 in mice, ribbons are found to be mostly round and smaller than in adult mice (Safieddine et al, 2012; Michanski et al, 2019). Also ribbons were observed first as floating structures, which during development attach to the active zone by two rootlets and finally upon hearing onset attach via one anchor (Sobkowicz et al, 1986; Safieddine et al, 2012; Michanski et al, 2019). The development of ribbons from various small, floating structures to a few oval shaped complexes attached to the active zone has been postulated to be a result of fusion events between ribbons during development (Michanski et al, 2019). Furthermore, a reduction in $\mathrm{Ca}^{2+}$ channels in the plasma membrane of IHCs is seen upon hearing onset (Michanski et al, 2019; Wong et al, 2014), which coincides with a change of activity pattern of the IHC from spontaneous action potentials to graded receptor potentials and with the loss of direct efferent innervation of IHCs (Safieddine et al, 2012; Johnson et al, 2011). Interestingly, OHCs were also found to form ribbon synapses with type I spiral ganglion neurons in early developmental stages (PO - P6). Nevertheless, these contacts disappear with the onset of hearing and $\mathrm{OHCs}$ are solely innervated by type II ganglion neurites (Sobkowicz et al, 1986; Huang et al, 2012), leaving IHCs to be the only cells forming ribbon synapses in the Organ of Corti.

\subsection{The process of synaptic transmission at the IHC ribbon synapse and its molecular components}

In IHCS, the ribbon tethers a monolayer of synaptic vesicles close to the active zone and thereby organizes vesicle populations at the ribbon synapse (Lenzi et al, 1999; Chakrabarti et al, 2018). A subset of these ribbon-associated vesicles are found in close proximity to the 
active zone membrane and are thought to represent docked vesicles (Figure 2B), which have been interpreted as the readily releasable pool (RRP) of synaptic vesicles (analogous to conventional synapses; Moser \& Beutner, 2000). These are the first vesicles to be released upon short stimulation and can be quantified by membrane capacitance measurements (Moser \& Beutner, 2000). The ribbon has been reported to tether $100-200$ vesicles (Khimich et al, 2005), of which about 14 are also docked to the active zone and therefore comprise the RRP (Moser \& Beutner, 2000; Liberman, 1980; Saito, 1980). This first component of exocytosis shows a very fast depletion within a few milliseconds. With ongoing stimulation, exocytosis of a pool of slower releasing vesicles takes place, which can be maintained over seconds of stimulation, with nearly constant rates (Nouvian et al, 2006; Moser \& Beutner, 2000). The fact that the ribbon synapse of IHCs is capable to do so, indicates the presence of an efficient vesicle replenishment mechanism in order to compensate the depletion of the vesicle pool that is competent to be exocytosed. Vesicle resupply indeed has been shown to be fast with 1.9 vesicles/ms (Griesinger et al, 2005). In cone photoreceptors, the presence of the ribbon itself has been proposed to play a role in the efficiency of sustained vesicle exocytosis by preventing the depletion of vesicles in the first place (Jackman et al, 2009). However, the exact function(s) of the ribbon is not clear yet, since it has also been hypothesized to be important for vesicle priming (preparing the vesicle for fusion; Snellman et al, 2011) and support in synchronous release of multiple vesicles at one active zone (Graydon et al, 2011). A more detailed knowledge of the proteins present at the ribbon synapse would probably provide a better understanding of its functional processes, including the synaptic vesicle recycling process.

As mentioned before in conventional synapses the molecular components of the synaptic vesicle recycling process are well understood (Figure $2 \mathrm{~A}$ ). The synaptic vesicle exocytosis process in neurons is $\mathrm{Ca}^{2+}$-dependent and starts with the opening of voltage-gated $\mathrm{Ca}^{2+}$ channels in the presynaptic terminal upon stimulation (Südhof, 2004). The local $\mathrm{Ca}^{2+}$ concentration at the active zone is increased and $\mathrm{Ca}^{2+}$ is bound by synaptotagmin 1 , a very well-studied $\mathrm{Ca}^{2+}$ sensor, which mediates the fusion of docked and primed synaptic vesicles by interacting with the plasma membrane and the SNARE (soluble N-ethylmaleimidesensitive-factor attachment receptor) complex (Rizzoli, 2014). The vesicle docking process involves the interaction of the vesicle associated molecule Rab3 and Rab3-interacting molecules (RIMs) (Haucke et al, 2011), to bring the vesicle close to the active zone. The scaffolding proteins of the active zone, bassoon and piccolo, are probably also involved in the docking process (Rizzoli, 2014; Hallermann et al, 2010). Priming of the synaptic vesicle involves making the vesicle competent for fusion by interaction of the vesicle SNARE molecule (synaptobrevin 2 or VAMP2) with the plasma membrane associated SNARE molecules (syntaxin 1 and SNAP25), which form a partially coiled complex (Rizzoli, 2014). Other proteins, like complexin, Munc13 and Munc18, help in the priming process by stabilizing the complex (Jahn \& Fasshauer, 2012; Ma et al, 2013). Fusion of the vesicle membrane and the plasma membrane is achieved by the complete formation of the coiled coil structure between the SNARE molecules. This results in the collapse of the vesicle into the plasma membrane and neurotransmitter release (Rizzoli, 2014; Südhof, 2004) into the 
synaptic cleft, where they can be bound by receptors in the postsynaptic membrane.

In IHC ribbon synapses, it is thought that the principle of vesicle fusion is the same, although details about individual steps and the identity of proteins involved in those steps are mostly unclear (Figure 2B). Only a few components of the synaptic vesicle exocytosis machinery are well understood. The process is known to be $\mathrm{Ca}^{2+}$ dependent, like in conventional synapses, but the IHC ribbon synapses mainly express Cav1.3 L-type $\mathrm{Ca}^{2+}$ channels, instead of $\mathrm{N}$-type $\mathrm{Ca}^{2+}$ channels in conventional synapses (Brandt et al, 2003; Zhang et al, 1999; Robertson \& Paki, 2002; Dou et al, 2004; Rodriguez-Contreras \& Yamoah, 2001). Often, it is not clear, whether the process of synaptic vesicle exocytosis in hair cell ribbon synapses involve the same proteins as in conventional synapses. A few proteins have been shown to be identical or at least similar, like the scaffolding proteins bassoon, which anchors the ribbon to the active zone (Dick et al, 2001, 2003; Khimich et al, 2005) and piccolo, which is expressed as a shorter isoform piccolino in IHC ribbon synapses (Regus-Leidig et al, 2013; Dick et al, 2001). Some of the differences in protein composition between the ribbon synapse and the conventional synapse are well studied, like e.g. the expression of ribeye as major component of the ribbon (Schmitz et al, 2000; Kantardzhieva et al, 2012; Becker et al, 2018) or the expression of otoferlin, which has been studied extensively and has been reported to compensate for the lack of synaptotagmin 1 as a $\mathrm{Ca}^{2+}$ sensor in IHC ribbon synapses (Roux et al, 2006; Yasunaga et al, 2000; Pangršič et al, 2010; Takago et al, 2018; Michalski et al, 2017; Safieddine \& Wenthold, 1999; Uthaiah \& Hudspeth, 2010; Wenthold et al, 2002). It is also known that synaptophysin and synapsin are not expressed in the IHC ribbon synapse (GilLoyzaga \& Pujol, 1988; Safieddine \& Wenthold, 1997; Mandell et al, 1990). Furthermore, synaptic vesicles of the IHC contain the glutamate transporter vGlut3 instead of vGlut1/2, like in conventional synapses (Seal et al, 2008). Nevertheless, many other exocytosis proteins remain not sufficiently studied or controversially discussed. One of the best known examples for this is the discussion about the presence or absence of conventional neuronal exocytosis SNARE proteins (VAMP2, syntaxin 1 and SNAP25) in the IHC synapse. On the one hand some studies report the expression of these SNAREs and on the other hand some studies state that these proteins cannot be detected at the IHC ribbon synapse (Nouvian et al, 2011; Safieddine \& Wenthold, 1999; Uthaiah \& Hudspeth, 2010; Wenthold et al, 2002). Furthermore, it is unclear which proteins functionally compensate for the lack of those proteins that have been shown to not be expressed at the ribbon synapse, like synaptophysin and synapsin.

Components of the synaptic vesicle endocytosis process seem to be more conserved between conventional synapses and IHC ribbon synapses. In conventional synapses, recycling of the vesicle components (proteins and membrane lipids) that have been released into the plasma membrane is mainly achieved by clathrin-mediated endocytosis (Figure 2A; Südhof, 2004; Haucke et al, 2011; Rizzoli, 2014). Synaptic vesicle proteins in the plasma membrane are recognized by adaptor proteins like AP2 and AP180, which in turn recruit the clathrin coat molecules, clathrin light chain and clathrin heavy chain (Diril et al, 2006; Koo et al, 2011; Haucke et al, 2011). Membrane curvature is induced by proteins like amphiphysin 
and endophilin (McMahon \& Gallop, 2005), which also recruit the GTPase dynamin (Slepnev $\&$ De Camilli, 2000). The coated vesicle is finally pinched off the plasma membrane by the help of the GTPase activity of dynamin (Hinshaw, 2000; Faelber et al, 2012). The subsequent shedding of the clathrin coat is mediated by the proteins HSC70 and auxilin (Rizzoli, 2014). The newly retrieved synaptic vesicle is then either directly refilled with neurotransmitter (mediated by the proton pump vATPase) or first targeted by endosomal sorting (discussed in Rizzoli, 2014 and Südhof, 2004).

Commonly known key proteins of the synaptic vesicle endocytosis process, like clathrin, dynamin, AP2 and amphiphysin, have been shown to be present at the IHC ribbon synapse as well (Neef et al, 2014; Jung et al, 2015). This indicates that clathrin-mediated endocytosis is at least part of the synaptic vesicle recycling in ribbon synapses. Nevertheless, the entirety of proteins involved in that process is not identified yet and it is still under discussion whether clathrin-independent endocytosis plays a role in synaptic vesicle recycling and whether the synaptic vesicle undergoes an endosomal sorting process.

In summary, some specific details about the synaptic vesicle recycling process at the IHC ribbon synapse are well understood, whereas other details have not been subject to much investigation or have been studied intensively, but are still not entirely understood.

A

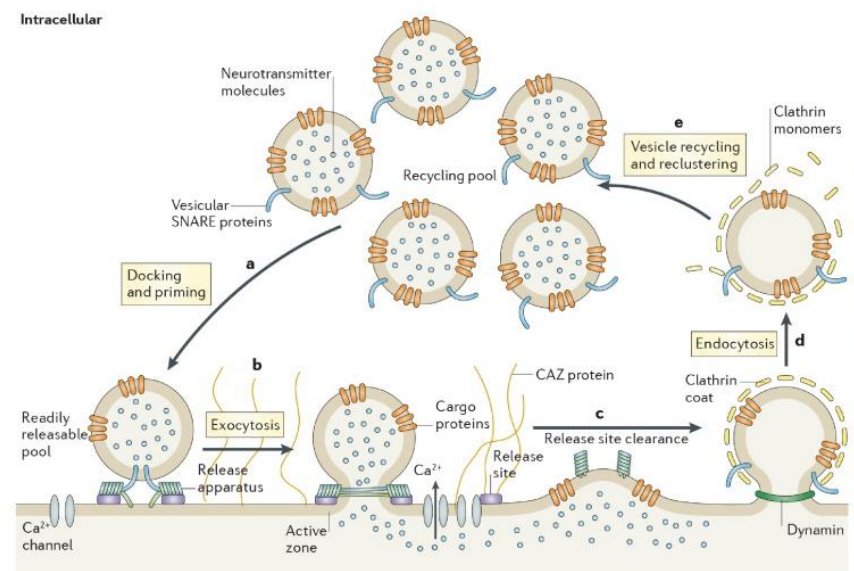

B

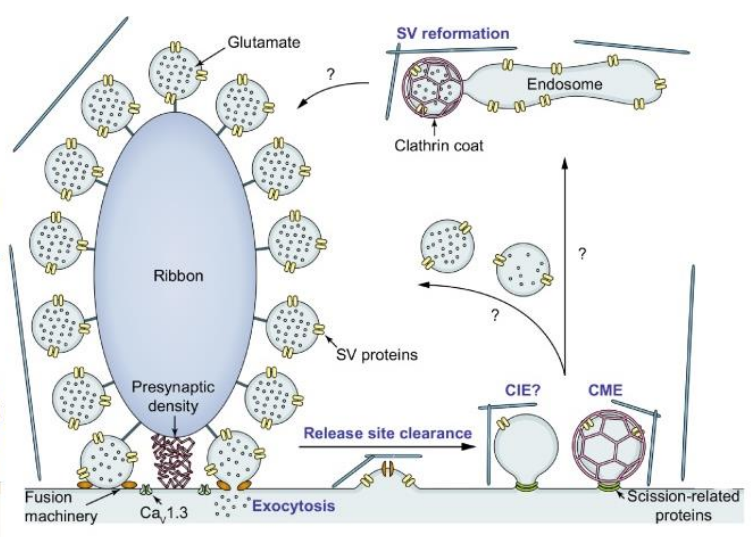

Figure 2 The synaptic vesicle recycling process in conventional synapses and the IHC ribbon synapse.

A) Steps and molecular components of the synaptic vesicle recycling process in conventional synapses, like the synapse of hippocampal neurons, are well understood. The synaptic vesicle passes through a docking and priming procedure, which involves key proteins like the SNARE proteins syntaxin1, SNAP25 and VAMP2. Upon stimulation, the resulting $\mathrm{Ca}^{2+}$ influx is sensed by synaptotagmin and vesicle fusion is initiated, which is mediated by the SNARE complex. Neurotransmitters are released and the vesicle collapses into the plasma membrane for full fusion. Synaptic vesicle components are recycled by clathrin-mediated endocytosis, involving proteins like AP2, AP180, endophilin, amphiphysin and dynamin. After uncoating, the synaptic vesicle is either refilled with neurotransmitter or first undergoes an endosomal sorting process. Reprinted by permission from Springer Nature: Springer Nature; Nature Reviews Neuroscience; 'Protein scaffolds in the coupling of synaptic exocytosis and endocytosis' by Volker Haucke, Erwin Neher, Stephan J. Sigrist (C) 2011 Macmillan Publishers Limited. All rights reserved B) In the ribbon synapse of IHCs, a few details of the recycling process are well investigated, like the tethering of synaptic vesicles to the ribbon and therefore close to the active zone and the distribution of $\mathrm{Ca}^{2+}$ channels. Nevertheless, 
many open questions still exist, mainly concerning protein compositions and exact functions of individual proteins. Adapted by permission from Wiley: FEBS Letters; 'Balancing presynaptic release and endocytic membrane retrieval at hair cell ribbon synapses' by Tina Pangršič and Christian Vogl; (C) 2018 Federation of European Biochemical Societies.

\subsection{The need for improvements in existing techniques for the investigation of synaptic proteins at the IHC ribbon synapse}

One cause for the insufficient knowledge of proteins involved in the synaptic vesicle recycling process at the $\mathrm{IHC}$ ribbon synapse is the difficulty in investigating that process. Biochemical methods usually used to identify and quantify proteins, like mass spectrometry (MS) or co-immunoprecipitation, face major problems with contamination from other synapses, due to the lack of sufficient purification protocols for ribbon synapses. Moreover, these techniques lack spatial resolution and discrimination of single synapses, due to the need for pooling of all synapses from one or multiple samples in order to provide a high enough sample quantity. Since IHC numbers per Organ of Corti are limited, they do not provide enough material to run reliable analyses in MS. On the other hand, investigations based on imaging techniques, like fluorescence microscopy or electron microscopy, provide excellent spatial resolution, but often face problems of sub-optimal sample preparation (resulting in artifacts) and labelling strategies. Thus, improvements in existing sample preparation and labelling efficiency can provide an opportunity to investigate expression, abundance and localization of proteins in IHCS in more detail. I have achieved such an improvement by establishing an alternative fixation procedure, which helps to enhance immunostainings of cells and tissues. Furthermore, I have utilized this improvement in sample preparations for the estimation of protein copy numbers, by a technique based on immunofluorescence imaging (further described below). These improvements can be especially useful to confirm the presence or absence of specific proteins at the hair cell ribbon synapse. An estimation of protein abundance can provide insight into whether a protein might be abundant enough to contribute to a specific functional process, like exo- or endocytosis. Precise knowledge about where a certain protein is located might also indicate in which functional process the protein is involved or where a certain process might take place. In the case of the synaptic vesicle recycling process in IHCs, this can be useful to understand whether endocytosis of recycling vesicles is achieved directly at the ribbon and close to the active zone or at farther distance after release site clearance. Ideally, such localization studies of specific proteins of interest (POI) can be combined with markers for specific functional processes, to be able to assign a POI to a certain process. This has been done for decades in conventional synapses, e.g. to investigate the location of synaptic vesicle proteins in the plasma membrane upon exo- and endocytosis (Hoopmann et al, 2010; Opazo et al, 2010; Hua et al, 2011; Truckenbrodt et al, 2018b). Here, many specific markers are available and labeling as well as imaging techniques can be applied easily. The application of these combined techniques in IHCs however is still challenging. 


\subsection{Improvement of the fixation method for super resolution imaging studies}

The most commonly used fixation technique implemented for samples investigated by immunohisto- or cytochemistry based imaging studies, is chemical fixation with a $4 \%$ formaldehyde solution (PFA). Chemical fixation by PFA is based on the crosslinking of amine containing molecules (like proteins with their peptide-bonds and amine containing amino acid side chains) via the formation of covalent bonds. The usage of formaldehyde as a fixative for cells and tissues has been a method, which provided sufficient preservation of samples for decades, until the development of imaging techniques with substentially increased resolution (Hell \& Wichmann, 1994; Eggeling et al, 2015) highlighted the need for improvement. The increase of imaging resolution made artifacts visable, which were caused by improper fixation. These fixation artifacts include incomplete sample preservation, morphological changes, interference with epitope recognition for immunolabeling, mislocalization of target proteins and slow and incomplete fixation kinetics and have been reported numerous times in the past (Melan, 1994; Schnell et al, 2012; Tanaka et al, 2010). Schnell and collegues for example showed that formaldehyde fixation and subsequent immunostaining in comparison to GFP-labeling and live imaging can cause extensive mislocalization of the target protein and that fixation interferes with the localization of epitopes (Schnell et al, 2012). Another study implementing single molecule tracking after fixation, impressively showed that even after 30 or 90 min of PFA fixation, a substential amount of membrane proteins still shows lateral mobility (Tanaka et al, 2010). Artificial clustering of target proteins is another important issue for immunolabeling experiments, which is highlighted by the emergence of super resolution microscopy. While certain labeling patterns in epifluorescence or confocal microscopy might not seem out of the ordinary, in super resolution imaging one can often observe "dotty" patterns, which can be a result of antibody clusters rather than specific staining signals (Opazo et al, 2010; Maidorn et al, 2016). This might be caused by insufficient fixation and clustering of target proteins induced by polyclonal antibodies.

Due to these problems with PFA fixation, other fixatives have been used as alternatives in the past. Among these are e.g. picric acid, methanol and glutaraldehyde. While picric acid fixes proteins by coagulation (formation of salts), methanol fixation (and alcohol based fixation in general) is achieved by denaturating proteins and therefore precipitating them. Both techniques are used only for the fixation of specific samples, such as glycogen and microtubules, respectively, due to major limitations. Picric acid has been reported to hydrolyse nucleic acids, cause sample shrinkage and is overall a serious safety issue, since it is highly toxic and explosive. Methanol is know to extract membrane lipids and thus cannot be used for the fixation of membrane assosciated proteins; it additionally increases the risk of washing out target proteins from cells during the staining process (Carson, 2007; Eltoum et al, 2001; Drury \& Wallington, 1980; Hopwood, 1985; Latendresse et al, 2002). Glutaraldehyde, like formaldehyde, fixes proteins via chemical crosslinking, but in a more efficient way (Tanaka et al, 2010; Smith \& Reese, 1980), probably via a higher level of crosslinking, due to more groups availabe for chemical reaction. Nevertheless, glutaraldehyde exhibits a major drawback as well: the reduction of antigenecity of the fixed 
sample. This poses a substential problem for the use of those samples in immunostainings, since most epitopes cannot be labeled anymore. This is probably due to denaturation and reduced penetration of the sample by antibodies (Farr \& Nakane, 1981).

During our search for a fixative that does not exhibit the problems mentioned above and at the same time still allows efficient immunolabeling, we tested the molecule glyoxal. Glyoxal is the smallest di-aldehyde possible and therefore most likely fixes proteins under the same principle of chemical crosslinking like formaldehyde does (Figure 3). However, it has been reported to work faster and more effectively, when used with an accelerator like ethanol and that cross-linking capacity can be controlled via the $\mathrm{pH}$ of the glyoxal solution (Dapson, 2007).
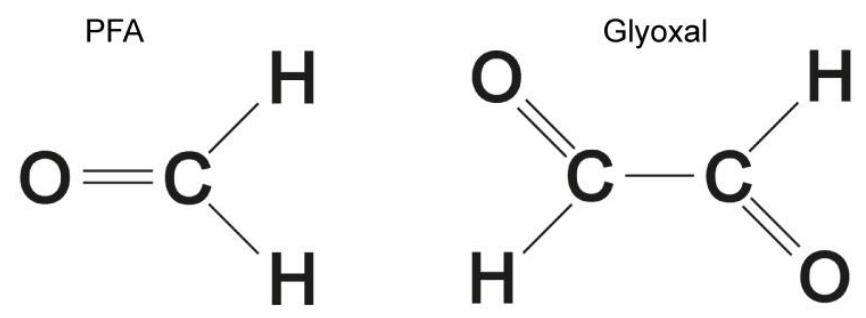

Figure 3 Structure of the molecules formaldehyde and glyoxal.

Formaldehyde (PFA) consists of a single aldehyde group and glyoxal is the smallest di-aldehyde, solely consitsing of two aldehyde groups. Both molecules can be used as fixatives.

Glyoxal has already been described to function as an alternative fixative to formalin for histological preparations in 1943 (Wicks \& Suntzeff, 1943). It was reported to pose less health risks and in a comparison of a $2 \%$ glyoxal solution with a $4 \%$ formalin solution it was found to perform well for the fixation of small histology samples. Especially in histology there is a great need for an alternative fixative to formalin, which is highly toxic and irritating to nose and eyes. Moreover, immunohistological studies often depend on antigen retrieval after fixation with formalin, due to alterations in the tissue. Therefore, various different glyoxal based fixatives have been tested over the years in order to find a suitable replacement for formalin (Wicks \& Suntzeff, 1943; Umlas \& Tulecke, 2004; Sabatini et al, 1963; Paavilainen et al, 2010; Titford \& Horenstein, 2005). Among these tested fixatives were also commercially available glyoxal solutions, like Glyo-Fixx (Thermo Fisher \#10249908), which has been available for years, but is not commonly used in the scientific community. All studies, testing commercially available or self-made glyoxal solutions, provided rather heterogeneous results. While Wicks and Suntzeff reported glyoxal to be favourable to formalin for the fixation of small tissues, Umlas and Tulecke described glyoxal to be inferior to formalin in fixation and immunohistochemical stainings of breast speciments. In contrast to formalin, glyoxal fixation showed increased calcium solubility, which hindered the detcetion of microcalcification. Furthermore, glyoxal fixation did not alleviate the need for antigen retrieval for the detection of estrogen receptors in these samples (Umlas \& Tulecke, 2004). Yet another study compared glyoxal with other fixatives, like glutaraldehyde and formaldehyde, in enzyme histochemistry and electron microscopy experiments (Sabatini et al, 1963). Preservation in electron microscopy was found to be moderate to good for glyoxal in comparison to best preservation achieved with 
glutaraldehyde (which is still commonly used for EM preparations). Furthermore, enzyme activity was maintained in a moderate amount after glyoxal fixation, which was not the case for most of the other tested compounds. This highlights the often observed trade-off between optimal preservation and maintenance of the functionally active structure of the fixed proteins. A quite extensive test of different fixatives (among these Glyo-Fixx) for immunodetection in tissue samples, cell preperations and protein lysates found glyoxal based fixation comparable to formalin for immunohistochemistry, but found it to retain proteins poorly for Western Blot analysis (Paavilainen et al, 2010). In another study, testing Glyo-Fixx and other formalin alternatives, fixation of histological samples was rated according to different histological parameters. Glyo-Fixx seemed to achieve good overall morphological preservation and was rated high for some parameters, like nuclear detail, but was not found to be performing as well as formalin (Titford \& Horenstein, 2005). Overall, glyoxal fixation was described at least as an acceptable fixative in almost all studies, but details about the quality of fixation were reported very differently. It is important to notice that the tested glyoxal solutions in all studies vary in composition and concentration. GlyoFixx for example was described to additionally contain ethanol, methanol and 2-propanol (Titford \& Horenstein, 2005), whereas the glyoxal used in Wicks \& Suntzeff, 1943 was diluted in water and only contained additional calcium carbonate. These differences in the composition of the glyoxal solutions might explain the different outcomes reported in past years, since optimal glyoxal fixation has been described to depend on specific parameters, like the addition of ethanol as accelerator, the concentration of glyoxal and the $\mathrm{pH}$ of the solution (Dapson, 2007). Despite the comparison of glyoxal fixation to formalin fixation in histology, glyoxal has not been investigated much for other applications. Once glyoxal has been used as fixative to detect hormones in the rat brain, using immunofluorescence experiments (Swaab et al, 1975), but other than that its use for fluorescence imaging samples is rare.

In order to investigate whether glyoxal fixation can improve fixation of samples used in modern imaging techniques, we tested fixation with a $3 \% \mathrm{v} / \mathrm{v}$ glyoxal solution, containing $\sim 20 \%$ ethanol as accelerator, less than $1 \%$ acetic acid and a $\mathrm{pH}$ between 4 and 5 . We included a variety of different samples, ranging from cultured cell preparations, over tissue samples to protein lysates, and different staining and imaging techniques in our comparisons of glyoxal fixation to standard 4\% PFA fixation. Live imaging of cultured cells during fixation with either glyoxal or PFA in the presence of propidium iodide or the membrane dye FM 143 (both cannot penetrate living cells), showed that penetration of cell membranes was much faster for glyoxal than for PFA, leading to faster and easier access for the fixative to the target structures. This faster penetration of cells is most likely due to the additional ethanol in the glyoxal solution, since addition of a similar amount of ethanol to PFA has the same effect for membrane penetration. Nevertheless, preservation of cell and organelle morphology was improved for glyoxal in comparison to PFA fixation and was not dependent on ethanol addition. We were able to show this by live cell imaging of the morphology of cultured cells in brightfield during fixation and by fluorescence imaging of labeled mitochondria markers during fixation. In vitro fixation of rat brain lysates and subsequent 
Western Blot analysis indicated that glyoxal crosslinks proteins more extensively than PFA, similar to the crosslinking seen by glutaraldehyde. This has let to the concern that, similar to observations made after glutaraldehyde fixation, glyoxal fixation might reduce the antigenecity of the target proteins, so that immunofluorescent labeling is hampered. These concerns proved to be wrong, since we found glyoxal fixation to even improve the immunostaining with subsequent super-resolution imaging of several targets in hippocampal neuron cultures. These observations were confirmed in multiple other laboratories, testing glyoxal fixation in comparison to PFA on various different samples and staining/imaging techniques. Fixation and immunostaining of samples like cultured cells, neurons, sepia fin tissue, mouse Organ of Corti and mouse olfactory system showed an improvement in immunolabeling intensity for a variety of targets tested. 51 out of 82 targets were stained better after glyoxal fixation and only 19 were stained worse than after PFA fixation. These results from different scientific groups allover the world highlights that glyoxal fixation can be useful as an alternative to PFA fixation especially for samples and targets, which have been difficult to fix and immunostain in the past.

\subsection{CosiQuant: an imaging-based technique for the estimation of protein copy numbers}

Knowing the copy number of a certain protein in a cell or cellular compartment enables us to make assumptions about its function and importance in a process. Knowledge about the abundance of each protein involved in a certain process for example identifies potential limiting factors and can help to understand the process and its kinetics.

Classical methods to determine such protein copy numbers are mostly based on biochemical techniques, like Western Blot or MS. All these methods are based on the comparison with a standard of known quantity. In Quantitative Western Blotting, samples that need to be analyzed are run on an SDS PAGE (sodium dodecyl sulfate polyacrylamide gel electrophoresis) and the intensities of the resulting bands are compared to the intensity of the band obtained from the standard. Here, this standard comprises the recombinant and purified version of the investigated protein, which is run on the same SDS PAGE in known amounts (Jahn et al, 1984; Wilhelm et al, 2014). The quantification of proteins in methods like iBAQ (intensity-based absolute quantification (Schwanhäusser et al, 2011)) or AQUA (absolute quantification approach, (Gerber et al, 2003)) are based on MS, i.e. the separation and identification of proteins or peptides by their mass to charge ratio. The AQUA technique uses a synthetized and isotopically labeled peptide from the protein of interest as standard. This isotopically labeled peptide in known amounts is analyzed with the target sample in MS and thus protein numbers can be determined by comparison of the measured abundance of the protein of interest with the standard peptide (Gerber et al, 2003). iBAQ is a label-free method that uses peptides that have been already quantified as internal standard. The intensities of all MS peaks resulting from one protein are summed up and divided by the number of theoretically observable peaks from that protein. Comparison with the standard of known amounts in a linear regression enables the user to calculate absolute protein copy numbers (Schwanhäusser et al, 2011; Lu et al, 2007; Malmström et al, 2009; Wilhelm et al, 2014). These techniques have been used successfully in the past for protein and mRNA 
quantification, but as described in subsection 2.4, those biochemical methods only provide averages over a high amount of sample cells that need to be pooled. Thus resolution and differentiation on a cellular level cannot be provided. Furthermore, MS-based techniques like $\mathrm{iBAQ}$ and AQUA proved to be quite variable, which leads to the necessity of multiple replicates, which in turn needs a high amount of target sample. Quantitative Western Blotting is more precise, but not as easy to implement for a larger number of proteins of interest, since a synthetized and purified version of each protein is needed. These limitations make the application of those biochemical methods for protein quantification difficult for samples that cannot be obtained in large amounts and/or cannot be purified enough, like the ribbon synapse of the IHC.

Therefore, we have established a technique to estimate protein copy numbers based on an imaging approach. We termed this method CosiQuant (comparative synaptosome imaging for semi-quantitative copy numbers) and it relies on the comparison of immunostaining signals obtained from biochemically characterized synaptosome preparations and a sample of interest. Synaptosomes are synaptic boutons purified from brain samples via Ficoll gradient centrifugation (Rizzoli et al, 2006; Nicholls \& Sihra, 1986). These synaptosomes, obtained from rat brain samples, have been intensively characterized in the past (Rizzoli et al, 2006; Wilhelm et al, 2014). Using quantitative Western Blotting and iBAQ, Wilhelm and colleagues determined protein copy numbers for over 1000 synaptic proteins (Wilhelm et al, 2014). Furthermore, they characterized the overall morphology of synaptosomes by electron microscopy and protein location by stimulated emission depletion microscopy (STED). Due to their extensive work on the imaging of proteins in synaptosomes and hippocampal neuron cultures, they also provide an optimized staining protocol for synaptosome proteins, including a list of validated antibodies (Wilhelm et al, 2014). The principle of CosiQuant for the estimation of protein copy numbers is based on this work. Synaptosomes and a sample of interest are immunostained and imaged in parallel for a target protein. The resulting fluorescent signal intensities can then be compared between the two samples. The protein copy numbers of the target protein in the sample of interest can be calculated based on the known copy numbers of the protein in synaptosomes and the staining intensity ratio between synaptosomes and sample of interest. For a proof of principle, we applied this technique to an already quite well-known sample, the cultured hippocampal neuron. Synaptosomes and hippocampal neuron cultures were immunostained for 10 synaptic proteins of interest and 2 marker proteins for the identification of synapses. A semiautomatic analysis in Matlab provided the signal intensities of the stained POIs per synapse/synaptosome, permitting the calculation of protein copy numbers in hippocampal neuron synapses. The resulting estimates for the protein copy numbers reflect the amount of synaptic vesicles in synaptosomes and hippocampal neuron synapses and the amount of the synaptic vesicle marker synaptophysin as one of the investigated proteins. Therefore, CosiQuant represents a method that can be used to estimate the copy numbers of proteins in samples, which are otherwise difficult or even impossible to quantify. We made it easier to apply this method in other laboratories, by providing average numbers of antibodies binding to synaptosome samples as a substitute to the relative staining intensities compared 
above. This way other laboratories can use the CosiQuant technique without having to stain the exact same synaptosome samples, which have been used to obtain the protein copy numbers in the first place. Instead, they can determine the average number of antibodies per stained structure in their sample of interest and compare these numbers with the ones we provided for synaptosomes.

\subsection{Aims of this work}

The overall aim of this work was to study the synaptic vesicle recycling process in IHC ribbon synapses, including a detailed investigation of the proteins involved in that process by determining their spatial organization and abundancy. This cannot be done easily in these cells, due to difficulties in biochemical analyses and efficient immunostaining procedures. Therefore, I needed to optimize existing staining and imaging techniques, which would allow me to investigate IHC ribbon synapses with super-resolution microscopy and provide an estimate for proteins involved in the synaptic vesicle recycling process. I achieved to do so by establishing glyoxal as an alternative fixative to PFA, providing better immunostaining quality for a variety of targets and by establishing an imaging-based technique to estimate protein copy numbers in samples that cannot be investigated by standard biochemical methods. I have implemented these improvements in my study of the IHC ribbon synapse and determined the location and copy number of 19 synaptic proteins potentially involved in the synaptic vesicle recycling process. This information is summarized in a preliminary model of the spatial organization and abundance of these proteins within the ribbon synapse. This model needs to be further refined, but the information about the structural organization of synaptic proteins in the ribbon synapse can already be used to make assumptions about the functional importance of the investigated proteins, like the identification of potential bottlenecks in the process of exo-and endocytosis of synaptic vesicles. Future analyses can add to this model and increase its accuracy over time. 


\title{
03 Glyoxal as an alternative fixative to formaldehyde in immunostaining and super-resolution microscopy
}

Katharina N. Richter ${ }^{+}$, Natalia H. Revelo ${ }^{+}$, Katharina J. Seitz, Martin S. Helm, Deblina Sarkar, Rebecca S. Saleeb, Elisa D’Este, Jessica Eberle, Eva Wagner, Christian Vogl, Diana F. Lazaro, Frank Richter, Javier Coy-Vegara, Giovanna Coceano, Edward S. Boyden, Rory R. Duncan, Stefan W. Hell, Marcel A. Lauterbach, Stephan E. Lehnart, Tobias Moser, Tiago F. Outeiro, Peter Rehling, Blanche Schwappach, Ilaria Testa, Bolek Zapiec, Silvio O. Rizzoli

\author{
${ }^{+}$These authors contributed equally to the work \\ EMBO J 2018 Jan 4; 37(1):139-159 \\ http://emboj.embopress.org/content/37/1/139.long \\ doi: 10.15252/embj.201695709
}

Author contribution of Katharina N. Richter:

- Design (together with Silvio O. Rizzoli) and performance of the experiments shown in the following figures: Figure $1 \mathrm{~A}$ and $\mathrm{B}$, Figure 2, Figure $3 \mathrm{~A}$ (together with Natalia $\mathrm{H}$. Revelo) and $B$ (together with Verena V. Klüver), Figure 4 (together with Martin S. Helm), Figure S1A and B, Figure S2, Figure S3, Figure S4, Figure S5, Figure S6, Figure S7A and B, Figure S8, Figure S9, Figure S10, Figure S11, Figure S12 (together with Martin S. Helm), Figure S13 (together with Martin S. Helm), Figure S14 (together with Martin S. Helm), Figure S15 (together with Natalia H. Revelo and Martin S. Helm), Figure S17, Figure S18, Appendix Table 1

- Preparation of the manuscript together with Silvio O. Rizzoli (and comments from all other authors)

This article has been reproduced with permission from EMBO Press/Wiley-VCH Verlag GmbH \& Co.

$\mathrm{KGaA}$ under open access copyright and licensing regulations

(https://creativecommons.org/licenses/by/4.0/legalcode). 


\section{Glyoxal as an alternative fixative to formaldehyde in immunostaining and super-resolution microscopy.}

Katharina N. Richter ${ }^{1,2,+}$, Natalia H. Revelo ${ }^{1, \S,+}$, Katharina J. Seitz ${ }^{1,3}$, Martin S. Helm $^{1,3}$, Deblina Sarkar ${ }^{4}$, Rebecca S. Saleeb ${ }^{5}$, Elisa D'Este ${ }^{6}$, Jessica Eberle ${ }^{7}$, Eva Wagner ${ }^{8,9}$, Christian Vog ${ }^{10,11}$, Diana F. Lazaro ${ }^{12,13}$, Frank Richter ${ }^{3,14}$, Javier CoyVegara $^{15}$, Giovanna Coceano ${ }^{16}$, Edward S. Boyden ${ }^{17}$, Rory R. Duncan ${ }^{5}$, Stefan W. Hell $^{6}$, Marcel A. Lauterbach ${ }^{7}$, Stephan E. Lehnart ${ }^{8,9}$, Tobias Moser ${ }^{10,11}$, Tiago F. Outeiro $^{12,13}$, Peter Rehling ${ }^{14,18}$, Blanche Schwappach ${ }^{15}$, Ilaria Testa ${ }^{16}$, Bolek Zapiec $^{19}$, Silvio O. Rizzoli ${ }^{1,2, *}$

${ }^{1}$ Department of Neuro- and Sensory Physiology, University of Göttingen Medical Center, Göttingen, Germany

${ }^{2}$ Cluster of Excellence Nanoscale Microscopy and Molecular Physiology of the Brain, Göttingen, Germany

${ }^{3}$ International Max Planck Research School Molecular Biology, Göttingen, Germany

${ }^{4}$ MIT Media Lab

${ }^{5}$ Edinburgh Super-Resolution Imaging Consortium, Institute of Biological Chemistry, Biophysics, and Bioengineering, Heriot-Watt University, Edinburgh, UK

${ }^{6}$ Department of NanoBiophotonics, Max-Planck-Institute for Biophysical Chemistry, Göttingen, Germany

7 Department of Neural Systems, Max-Planck-Institute for Brain Research, Frankfurt am Main, Germany

${ }^{8}$ Heart Research Center Göttingen, Department of Cardiology \& Pulmonology, University Medical Center Göttingen, Göttingen, Germany

${ }^{9}$ German Center for Cardiovascular Research (DZHK) Site Göttingen

10 Institute for Auditory Neuroscience and InnerEarLab, University Medical Center Göttingen, Göttingen, Germany

11 Max-Planck-Institute for Experimental Medicine, Auditory Neuroscience Group, Göttingen, Germany

${ }^{12}$ Department of Experimental Neurodegeneration, Center for Nanoscale Microscopy and Molecular Physiology of the Brain, Center for Biostructural Imaging of Neurodegeneration, University Medical Center Göttingen, Göttingen, Germany

${ }^{13}$ Max-Planck-Institute for Experimental Medicine, Göttingen, Germany

${ }^{14}$ Department of Cellular Biochemistry, University Medical Center Göttingen, Göttingen, Germany

${ }^{15}$ Department of Molecular Biology, University Medical Center Göttingen, Göttingen, Germany

${ }^{16}$ Department of Applied Physics and Science for Life Laboratory, KTH Royal Institute of Technology, Stockholm, Sweden

17 Departments of Brain and Cognitive Science and Biological Engineering, MIT Media Lab and McGovern Institute, Cambridge, MA, USA

${ }^{18}$ Max-Planck-Institute for Biophysical Chemistry, Göttingen, Germany

${ }^{19}$ Max Planck Research Unit for Neurogenetics, Frankfurt am Main, Germany

${ }^{\S}$ Present address: Department of Tumor Immunology, Radboud Institute for Molecular Life Sciences, Radboud University Medical Center, Nijmegen, the Netherlands

+ These authors contributed equally to this work

*Corresponding author. Tel: +49 551 395911; E-mail: srizzol@gwdg.de 


\subsection{Abstract}

Paraformaldehyde (PFA) is the most commonly used fixative for immunostaining of cells, but has been associated with various problems, ranging from loss of antigenicity to changes in morphology during fixation. We show here that the small dialdehyde glyoxal can successfully replace PFA. Despite being less toxic than PFA, and, as most aldehydes, likely usable as a fixative, glyoxal has not yet been systematically tried in modern fluorescence microscopy. Here we tested and optimized glyoxal fixation, and surprisingly found it to be more efficient than PFA-based protocols. Glyoxal acted faster than PFA, cross-linked proteins more effectively, and improved the preservation of cellular morphology. We validated glyoxal fixation in multiple laboratories against different PFA-based protocols, and confirmed that it enabled better immunostainings for a majority of the targets. Our data therefore support that glyoxal can be a valuable alternative to PFA for immunostaining.

\subsection{Introduction}

The $4 \%$ paraformaldehyde (PFA) solution has been a standard fixative for immunostaining and fluorescence microscopy, for several decades. Nevertheless, the literature contains numerous reports that PFA causes morphological changes, loss of epitopes, or mislocalization of target proteins, and that it fixes the samples slowly and incompletely (see for example Schnell et al, 2012; Tanaka et al, 2010; Melan, 1994). Many other fixatives have been introduced to alleviate these problems. Among them, glutaraldehyde is probably the most commonly used, since it fixes the samples faster and more completely than PFA (Smith \& Reese, 1980). Mixtures of PFA and glutaraldehyde result in accurate fixation, and reduce the lateral mobility of molecules (Tanaka et al, 2010), presumably by increasing the level of protein cross-linking. However, this fixative mixture also reduces the efficiency of immunostainings, by blocking the antibody access to epitopes, or by causing particular epitopes to unfold (Farr \& Nakane, 1981). Alcohol-based fixation, such as treatments with ice-cold methanol (Tanaka et al, 2010), results in stable fixation for a sub-population of cellular structures (such as microtubules), but leads to poor morphology preservation, and to a loss of membranes and cytosolic proteins. Overall, the improvements in fixation induced by glutaraldehyde or methanol do not compensate for their shortcomings, thus in most cases leaving PFA as the current fixative of choice.

A superior alternative to PFA is needed, especially since artifacts that were negligible in conventional microscopy are now rendered visible by the recent progress in super-resolution microscopy (nanoscopy) (Eggeling et al, 2015). To find a fixative that maintains high quality immunostainings while alleviating PFA problems, we have tested several compounds. We searched for commercially available molecules, which could be readily used by the imaging community. These included different combinations of PFA and glutaraldehyde, picric acid (Hopwood, 1985), and di-imido-esters (Woodruff \& Rasmussen, 1979) which, however, were 
not better than PFA in immunostaining experiments. We have also investigated different aldehydes. We avoided highly toxic compounds such as acrolein, which would not be easy to use in biology laboratories, and we also avoided large aldehydes (more than 4-5 carbon atoms), whose fixative properties are expected to mimic those of glutaraldehyde. The small dialdehyde glyoxal fits these two criteria, since it has a low toxicity (as already noted in the 1940's, (Wicks \& Suntzeff, 1943), and contains only 2 carbon atoms. Glyoxal is used, at low concentrations, in glycation and metabolism studies (Boucher et al, 2015), which ensures that it is commercially available. It can be used as a fixative, and has even been once described, in 1963, to provide better morphology preservation to formaldehyde (Sabatini et al, 1963). It is almost unknown in fluorescence experiments. We were able to find one publication, from 1975 (Swaab et al, 1975), in which glyoxal was used in immunofluorescence on brain samples, albeit followed by sample freezing, and by procedures that are not compatible with modern, high-quality microscopy. We could also find a few publications on histological stains using glyoxal (for example Umlas \& Tulecke, 2004; Paavilainen et al, 2010), which further encouraged us to test this compound.

We tested glyoxal thoroughly, in preparations ranging from cell-free cytosol to tissues, and by methods spanning from SDS-PAGE to electron microscopy and super-resolution fluorescence microscopy. We found that glyoxal penetrated cells far more rapidly than PFA, and cross-linked proteins and nucleic acids more strongly, leading to a more accurate preservation of cellular morphology. Despite the stronger fixation, glyoxal did not cause a reduction of antibody binding to the samples. On the contrary, the resulting images were typically brighter than those obtained after PFA fixation. The initial optimization work was performed in one laboratory (Rizzoli, University Medical Center Göttingen, Germany), and the results were independently tested in 11 additional laboratories/teams: Boyden (MIT Media Lab and McGovern Institute, Massachusetts, United States), Duncan (Heriot-Watt University, Edinburgh, UK), Hell (Max Planck Institute for Biophysical Chemistry, Göttingen, Germany), Lauterbach (Max Planck Institute for Brain Research, Frankfurt am Main, Germany), Lehnart (University Medical Center Göttingen, Germany), Moser (University Medical Center Göttingen, Germany), Outeiro (University Medical Center Göttingen, Germany), Rehling (University Medical Center Göttingen, Germany), Schwappach (University Medical Center Göttingen, Germany), Testa (KTH Royal Institute of Technology, Stockholm, Sweden), and Zapiec (Max Planck Research Unit for Neurogenetics, Frankfurt am Main, Germany). We conclude that the immunostainings performed after glyoxal fixation were superior for the majority of the samples and targets, with only a minority ( $10 \%)$ of the targets being less well preserved and/or revealed. 


\subsection{Results}

Glyoxal preserves the cellular morphology more accurately than PFA, and fixes proteins and RNAs more strongly

To determine the optimal conditions of glyoxal fixation, we tested its action at different $\mathrm{pH}$ values (Appendix Table 1). We found that glyoxal requires an acidic $\mathrm{pH}$, roughly between 4 and 5 , despite one previous study that suggests that it may also fix samples at a neutral $\mathrm{pH}$ (Sabatini et al, 1963). In addition, we found that the morphology of the samples was much improved upon addition of a low-to-medium concentration of alcohol (ethanol, 10-20\%), which may act as an accelerator in the fixation reactions. Removing the ethanol, or adjusting the $\mathrm{pH}$ above or below the 4-5 range, resulted in poor sample morphology (Appendix Table 1). $\mathrm{pH}$ values of 4 or 5 provided similar results for most of our experiments (results obtained at $\mathrm{pH} 5$ are shown in all figures, unless noted otherwise), and provided better morphology preservation for cultured neurons than PFA. We tested PFA at various $\mathrm{pH}$ values $(4,5$, and 7$)$, with or without ethanol, at room temperature or at $37^{\circ} \mathrm{C}$ (Appendix Table 1), without finding a condition where the morphology of the PFA-fixed samples consistently bettered that of glyoxal-fixed samples.

We then proceeded to compare PFA and glyoxal fixation quantitatively. We first tested the speed with which these fixative solutions penetrate the cell membrane, by monitoring the fluorescence of propidium iodide, a fluorogenic probe that binds nucleic acids, and cannot enter living cells (Davey \& Kell, 1996). PFA fixation allowed propidium iodide entry into cultured cells only after $\sim 40$ minutes, while glyoxal was substantially faster (Fig. 1A). The same was observed using the membrane-impermeant styryl dye FM 1-43 (Betz et al, 1992): glyoxal fixation enabled significant FM 1-43 penetration within 1-2 minutes (Fig. 1B). The difference in membrane penetration is probably due to the ethanol present in the glyoxal fixative, since the addition of ethanol to the PFA solution enhances its penetration into cells in a similar fashion (Appendix Fig. S1), albeit it did not improve immunostainings with PFA (Appendix Fig. S1; we would like to point out that low $\mathrm{pH}$ values, 4 and 5, also failed to improve PFA immunostainings, as shown in the same Appendix figure). In the same experiments, FM 1-43 addition enabled us to visualize endocytotic events that took place during PFA fixation. Such events could be observed in every fixed cell (Fig. 1B), and indicated that the cells were still active during PFA fixation, from the point of view of membrane trafficking. No such events could be detected during glyoxal fixation. 
A
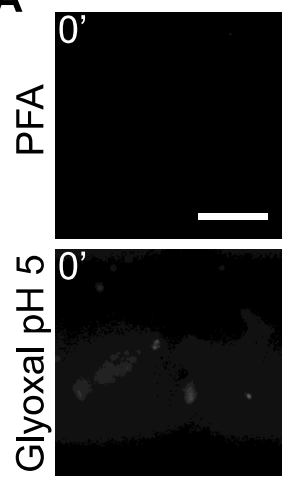

B
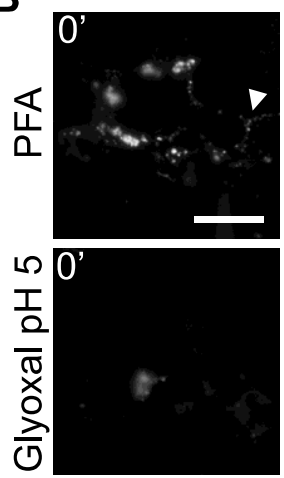
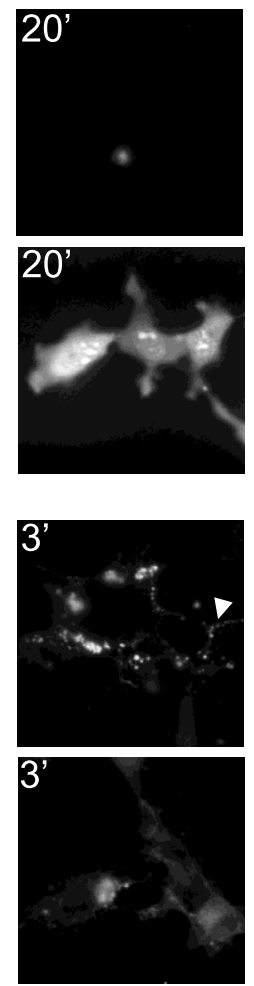
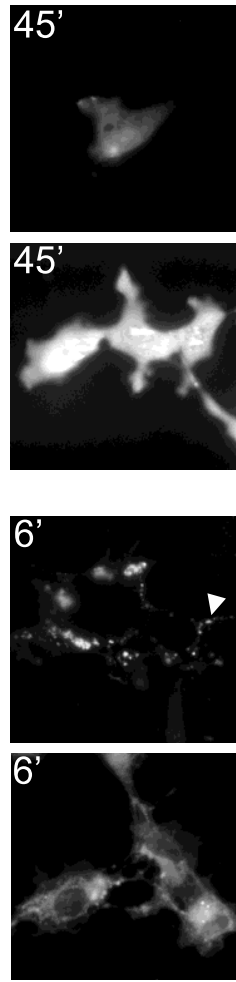
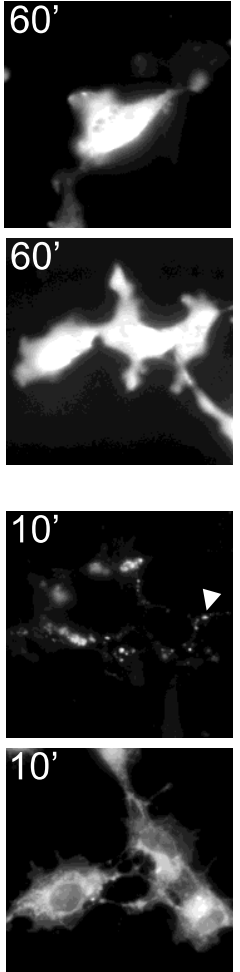
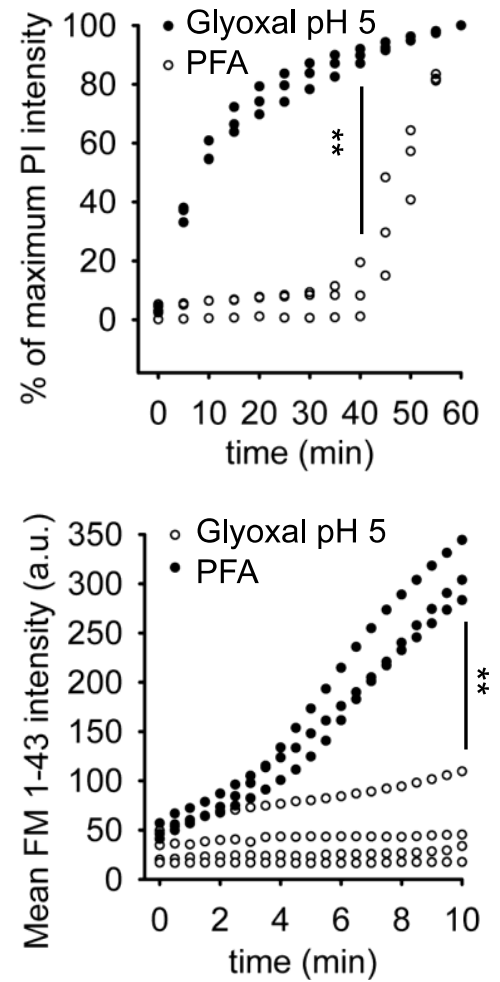

Figure 1 Comparison of cell penetration by PFA and glyoxal.

A Speed of propidium iodide (PI) penetration into fibroblasts during 60 min of fixation with either $4 \%$ PFA or $3 \%$ glyoxal. N = 3 independent experiments. Glyoxal fixation enables PI to penetrate far more rapidly into the cells.

B Speed of FM 1-43 penetration in similar experiments. The arrowhead points to one example of ongoing endocytosis during PFA fixation. $\mathrm{N}=3-4$ independent experiments. The general pattern of FM 1-43 entry was similar to that of propidium iodide. Only the first 10 minutes are shown, to enable an optimal observation of the kinetics of the first stages of FM 1-43 entry. The results parallel those obtained with PI: faster penetration during glyoxal fixation. Scale bar $=40 \mu \mathrm{m},{ }^{* *} p<0.01$

The hypothesis that cells were still partially active during PFA fixation, and less so in glyoxal fixation, was also confirmed by other experiments. First, we tested whether transferrin, which is readily endocytosed by a clathrin-mediated pathway, through the involvement of the transferrin receptor, is internalized during fixation. We applied fluorescently-conjugated transferrin onto cells during fixation with glyoxal or PFA (Appendix Fig. S2). We found that it was mainly fixed onto the plasma membrane by glyoxal, but that it was present both in the cells and on the membrane during PFA fixation (Appendix Fig. S2). Second, we tested whether the acidic lumen of the lysosome was maintained after fixation, by applying the probe LysoTracker (Appendix Fig. S3). Substantial LysoTracker labeling was observed after PFA fixation, but not after glyoxal fixation. Both of these experiments, therefore, indicate that glyoxal fixation stops cellular functions more efficiently than PFA.

The higher speed of membrane penetration seen with glyoxal was coupled to a better preservation of the general cell morphology, as observed by imaging cells during fixation (Fig. 2). PFA fixation was associated with the formation of membrane blebs and vacuoles, 
with organelle movement, and with a general change in the cell morphology (Fig. 2). Glyoxal fixation appeared to modify the cell morphology far less. This impression was confirmed by calculating the correlation coefficient between the initial cell images and images acquired at 5-minute intervals during fixation (Fig. 2). To obtain a similar view at the level of single organelles, we imaged the movement of endosomes labeled with fluorescently-conjugated transferrin or cholera toxin. As for the general cell morphology, glyoxal reduced the organelle movement more than PFA (Appendix Fig. S4).
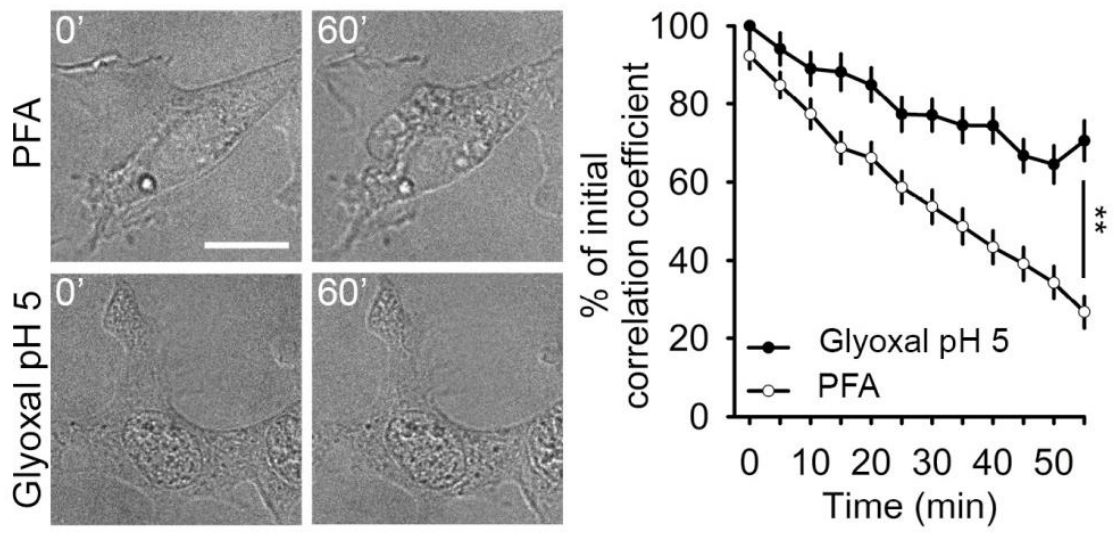

Figure 2 A comparison of morphological changes taking place during fixation with PFA or glyoxal. The changes were visualized by DIC images taken at 5 minute intervals during fixation. The graph shows the correlation of each image to the first frame. $\mathrm{N}=50$ (PFA) and 54 (glyoxal) cellular regions analyzed, from 3 independent experiments. The higher correlation value indicates that glyoxal preserves the initial cell morphology with higher accuracy than PFA. Scale bar $=20 \mu \mathrm{m}, * * p<0.01$ (two-sided Student's t-test)

We also monitored the morphology of mitochondria, which are known to become fragile during fixation. We visualized mitochondria in living cells, by tagging them with a GFP-linked reporter (TOMM70, Appendix Fig. S5), and imaged them again after fixation. Glyoxal preserved mitochondria at least as well as PFA. Moreover, ethanol addition to the PFA solutions worsened the preservation of mitochondria morphology, which suggests that ethanol does not improve PFA fixation, although it enhances its membrane penetration (Appendix Fig. S5). To test this issue further, we analyzed the correlation between the preand post-fixation images for fluorescent protein chimeras of a mitochondria reporter (TOMM70), a Golgi apparatus reporter (GalNacT2), a plasma membrane reporter (SNAP25), a cytoskeleton reporter (tubulin), and a vesicular reporter (synaptophysin). The correlations were similar among the two fixatives for TOMM70, GalNacT2, tubulin, and SNAP25. However, the pre- and post-fixation correlations in glyoxal fixed samples were higher for synaptophysin (Appendix Fig. S6), which marks the most mobile elements we investigated in this experiment (vesicles).

We then tested the protein cross-linking capacity of the different fixatives, by monitoring the proportion of the proteins that remained unfixed. We incubated brain cytosol samples with different fixatives for 60 minutes, and followed this by running the samples on 
polyacrylamide gels (Fig. 3A, Appendix Fig. S7). PFA, with or without ethanol addition, left $\sim 40 \%$ of the proteins unaffected (unfixed). Glyoxal (both $\mathrm{pH} 4$ and 5 ) reduced this unfixed pool to $\sim 20 \%$. Shorter fixation times reduced the amount of fixed proteins for all fixation conditions (Appendix Fig. S7). Glyoxal, both at $\mathrm{pH} 4$ or pH 5, fixed more proteins than PFA, PFA and ethanol or PFA at low pH, at all time points (Appendix Fig. S7).

The stronger fixation by glyoxal also applied for RNA molecules, albeit only at $\mathrm{pH} 4$, as observed by staining cells with propidium iodide after fixation (Fig. 3B). To test whether the glyoxal-fixed RNA molecules could still be detected by specific labeling, we performed fluorescence in situ hybridization (FISH) for a target that is often used as a standard in such experiments, glyceraldehyde 3-phosphate dehydrogenase (GAPDH). As for the propidium iodide staining, the GAPDH signal intensity was significantly raised by glyoxal at $\mathrm{pH} 4$ (Fig. 3B).
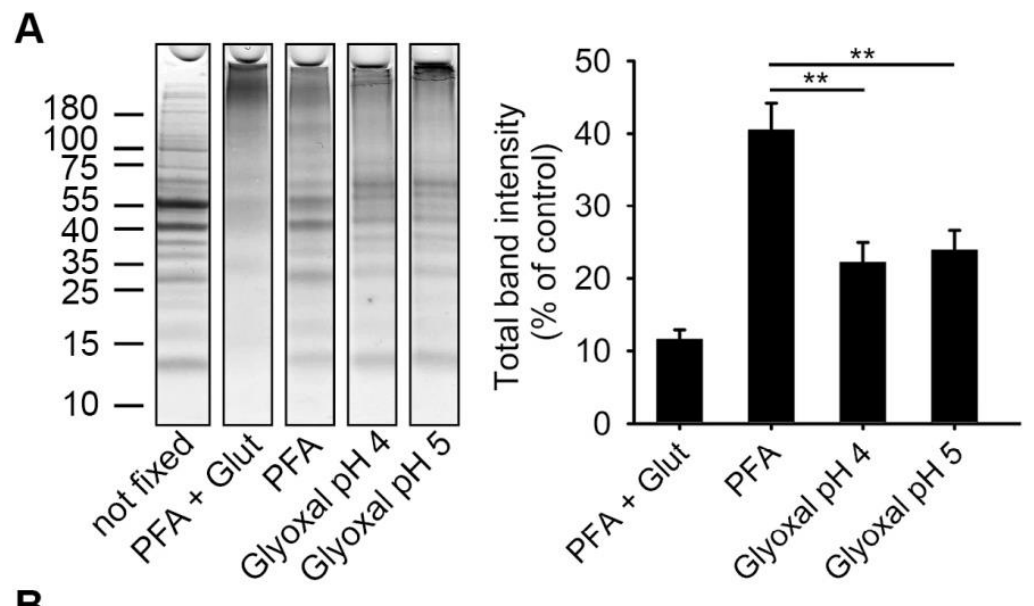

B

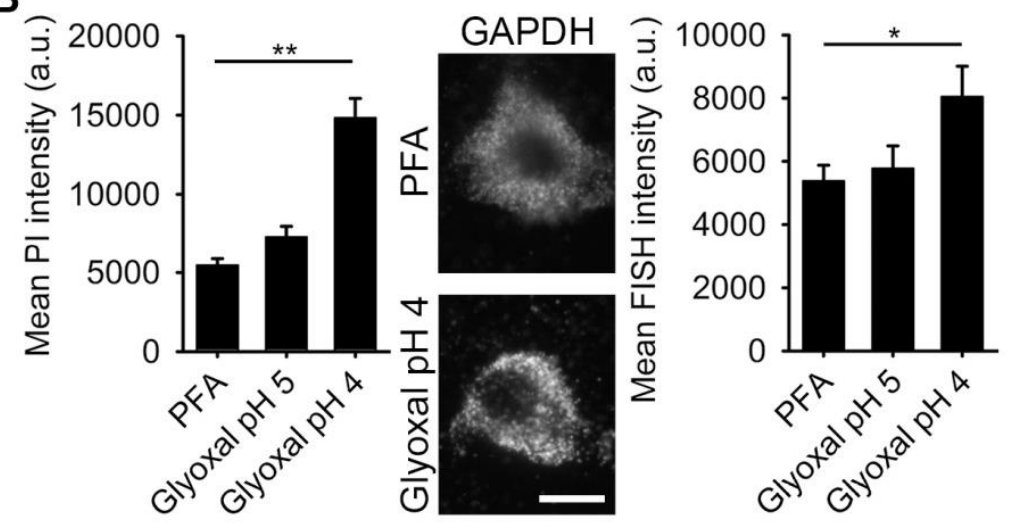

Figure 3 Comparison of protein and RNA fixation by PFA and glyoxal.

A SDS-PAGE gel showing rat brain cytoplasm incubated for 60 min with different fixatives. The graph shows the summed intensity of the bands in each lane. Fixed proteins either no longer run into the gel or form only smears. To compare the efficiency of fixation, the bands that survive fixation were summed and were expressed as \% of an unfixed control. The intensity of PFA-fixed samples was significantly higher than that of glyoxal-fixed samples ( $N=5$ independent experiments; one-way ANOVA with post hoc Tukey test). Glut $=0.2 \%$ glutaraldehyde.

B Staining of nucleic acids after fixation. The propidium iodide signal in fibroblasts was significantly higher for samples fixed with glyoxal $\mathrm{pH} 4(\mathrm{~N}=6-8)$. To test whether the fixed nucleic acids were still available for specific detection, we performed FISH for GAPDH in cultured neurons, using a standard protocol provided by the company Affymetrix. The fluorescence signal of the samples fixed with 
glyoxal ( $\mathrm{pH} 4$ ) was significantly higher than for PFA-fixed samples ( $\mathrm{N}=5-6$; two-sided Student's ttest). Scale bar $=10 \mu \mathrm{m}, * p<0.05, * * p<0.01$

To test whether similar effects apply also to lipids, we immunostained cultured cells for phosphatidylinositol-(4,5)- $\mathrm{P}_{2}\left(\mathrm{PIP}_{2}\right)$. The intensity of the immunostaining was substantially higher after glyoxal fixation (Appendix Fig. S8).

The stronger fixation induced by glyoxal could be a concern for experiments relying on enzymatic tags, such as the SNAP tag (Xue et al, 2015). Strong fixation may damage the enzyme, which would result in limited labeling. To test this, we expressed proteins coupled to the SNAP tag in cultured cells, fixed them with PFA or glyoxal, and then incubated them with a fluorophore that is bound by the SNAP tag, which couples to it covalently. The intensity of glyoxal-fixed samples was significantly higher than that of PFA-fixed samples (Appendix Fig. S9).

Glyoxal provides higher-quality STED images in immunostaining than PFA

Having verified that glyoxal is a faster and more effective fixative than PFA, we proceeded to investigate its efficiency in immunostaining. We expressed fluorescent protein chimeras of reporters for mitochondria, the Golgi apparatus, the plasma membrane, the cytoskeleton and vesicles, fixed the cells with PFA or glyoxal, and immunostained them. The immunostaining intensity of all of these structures, defined by the fluorescent protein signals, was significantly higher after glyoxal fixation (Appendix Fig. S10). To also test this without the expression of fluorescently tagged proteins, we immunostained cells that had been incubated with fluorescently labeled transferrin (Appendix Fig. S11). The transferrin is in this case present in endosomes, which should co-localize with endosomal markers such as EEA1. The co-localization was substantially higher after glyoxal fixation (Appendix Fig. S11).

We then focused on cultured neurons, which have been a standard preparation for nanoscopy (Willig et al, 2006; Xu et al, 2013), and found that the resulting images were often brighter (Fig. 4). We analyzed the intensity of the STED images, in terms of signal over background (Fig. 4), and determined that glyoxal indeed provided a higher signal for the large majority of the neuronal targets we investigated. 

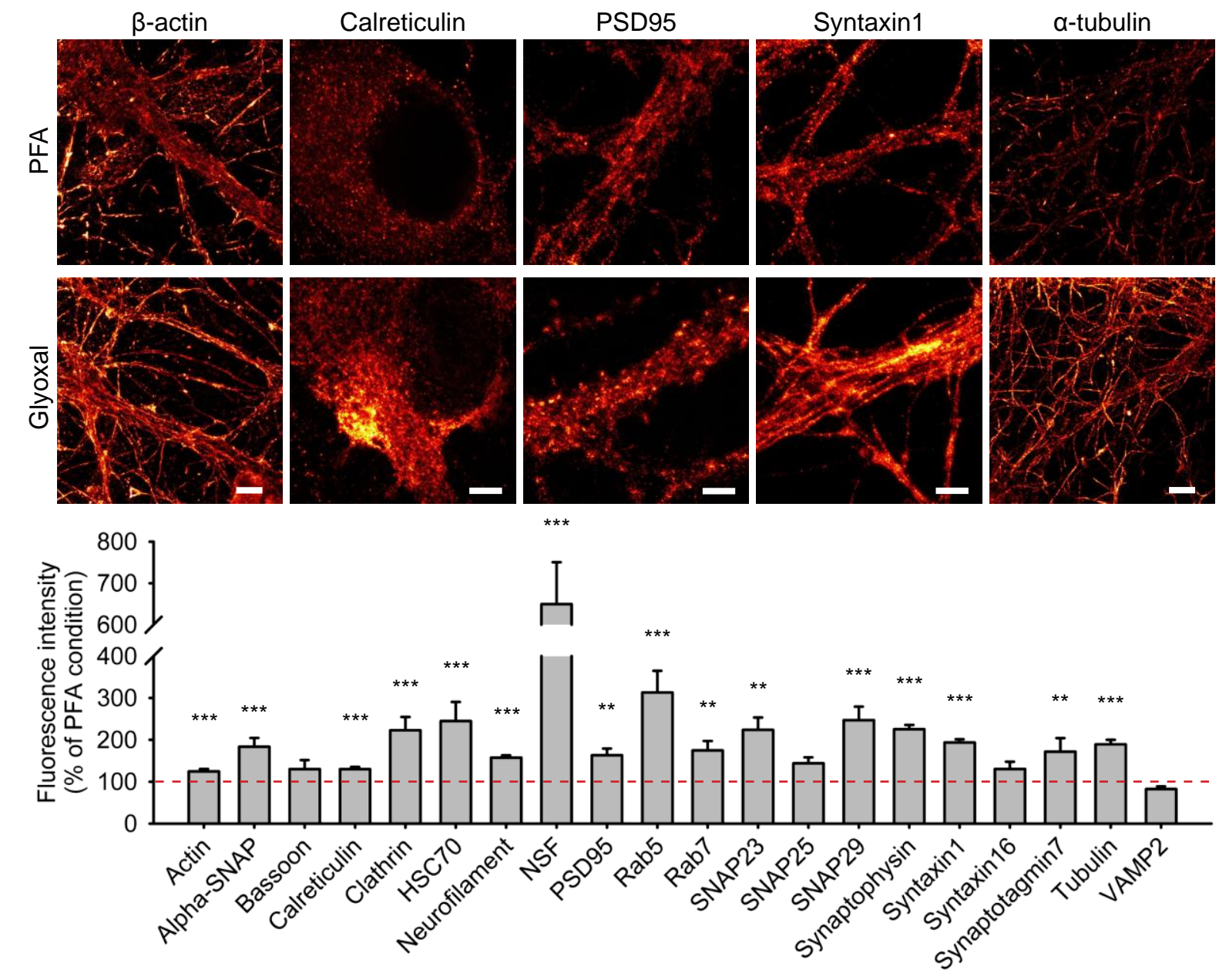

$* * *$

Figure 4 STED imaging of primary hippocampal neurons fixed with either PFA or glyoxal.

Strong differences in labeling patterns can be observed. The images are brighter and less "spotty" for the glyoxal-fixed samples. Structures such as filaments or organelles are more easily detected. Quantification of the fluorescence signal (fold over background) shows that 16 out of 20 stainings are significantly brighter in glyoxal fixed samples compared to the PFA fixed samples. $N=35-132$ objects. Scale bar $=6 \mu \mathrm{m}$ for $\beta$-actin and $\alpha$-tubulin, and $3 \mu \mathrm{m}$ for the other proteins. ${ }^{* *} p<0.01, * * *$ $\mathrm{p}<0.001$ (two-sided Student' t-test for PSD95, Wilcoxon rank-sum test for all other proteins).

When investigated by STED microscopy, the many images of PFA-fixed cells appeared dominated by isolated, uniformly distributed spots, which presumably represent antibody clusters (Opazo et al, 2012; Lang \& Rizzoli, 2010). The immunostaining signals appeared to be grouped in less uniform, more organelle-like structures after glyoxal fixation. To quantify this impression, an experienced user counted the number of organelle-like structures per $\mu \mathrm{m}^{2}$, in a blind fashion, for 20 targets immunostained in neurons. This provided a quantitative (albeit user-driven) measurement of the accuracy of the stainings (Appendix Fig. $\mathrm{S} 12)$. This analysis suggested that the immunostainings performed after glyoxal fixation more readily allow the identification of organelles, for the majority of the targets. This impression was confirmed by several additional analyses (see Appendix Fig. S13 and S14). 
A possible cause for the appearance of isolated, uniformly distributed spots in the PFA-fixed samples is the loss of some of the unfixed soluble molecules after PFA fixation, through diffusion into the extracellular space. As indicated in Fig. 3, 40\% of the proteins remained unfixed, and could therefore diffuse from the samples. To further test this hypothesis, we analyzed hippocampal neurons in electron microscopy, after fixation with PFA or glyoxal (Appendix Fig. S15). The cytosol appeared clearer (less electron-dense) in the PFA-fixed samples. In contrast, the glyoxal-fixed samples had a more electron-dense cytosol, which rendered them similar, at least superficially, to samples prepared by high-pressure freezing (Appendix Fig. S15).

We concluded, so far, that glyoxal appeared to be more efficient than PFA in several ways, such as speed and morphology preservation, which rendered it a better fixative for immunostaining and nanoscopy. Albeit we focused so far mostly on cell cultures, we also tested glyoxal in tissue preparations, where it enabled us to perform accurate immunostainings, in both Drosophila and mouse (Appendix Fig. S16, S17, and S18). We did not observe any difficulties in the antibody penetration in such tissues, in contrast to fixation by, for example, glutaraldehyde (as discussed in the Introduction).

\section{Glyoxal provides higher-quality images in immunostaining for many different laboratories}

The glyoxal fixation procedure established above was then tested in 11 different laboratories, in four countries (Germany, Sweden, UK, USA). We present the results in alphabetical order.

The Boyden laboratory (MIT Media Lab and McGovern Institute, Departments of Brain and Cognitive Science and Biological Engineering, Cambridge, Massachusetts, United States) tested Nucleoporin160 in conventional immunostainings of cell cultures, and found that fixation with glyoxal at $\mathrm{pH} 4$ resulted in brighter images than those obtained with PFA fixation. The samples exhibited similar morphology (Fig. 5). 

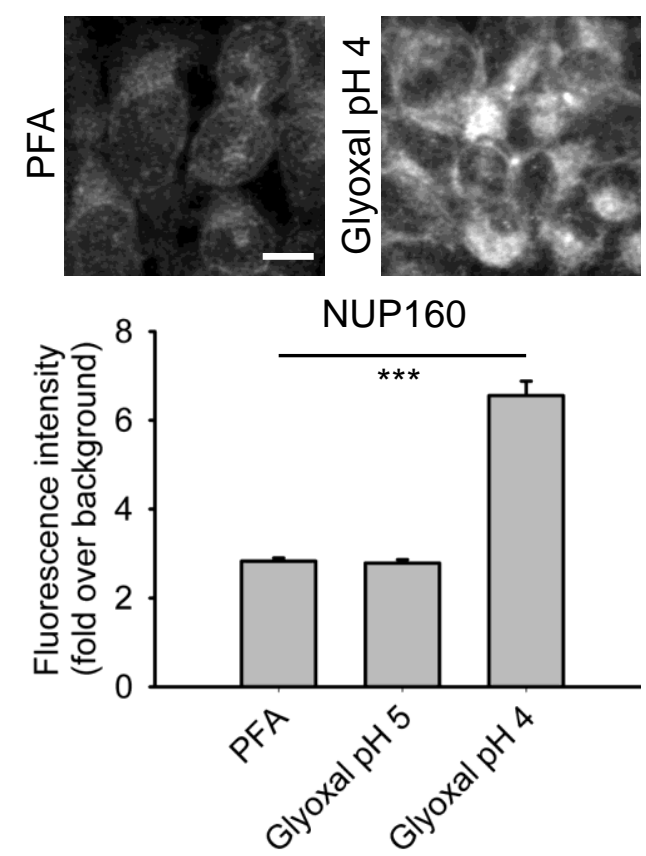

Figure 5 Comparison of immunostaining NUP160 after fixation with either PFA or glyoxal.

HeLa cells were stained for the nucleoporin complex protein NUP160 after fixation with either PFA, glyoxal pH 4 or glyoxal pH 5. Fluorescence intensities (fold over background) were compared and are shown in the graph. The quantification of fluorescence signals shows that glyoxal pH 4 fixation allows for significantly brighter stainings. $\mathrm{N}=73-156$ cells per condition analyzed. Scale bar $=10 \mu \mathrm{m} .{ }^{* * *} \mathrm{p}$ $<0.001$ (Wilcoxon rank-sum test).

The Duncan laboratory (Edinburgh Super-Resolution Imaging Consortium, Institute of Biological Chemistry, Biophysics, and Bioengineering, Heriot-Watt University, Edinburgh, UK.) also used conventional immunostainings of cultured cells (AtT20 cells), and analyzed the SNARE proteins syntaxin1 and SNAP25 and the autophagy marker LC3B. The immunostainings of the two SNARE proteins were brighter after glyoxal fixation (Fig. 6), whereas LC3B staining was brighter after PFA fixation. The morphology of the cells appeared similar for the two fixatives. 

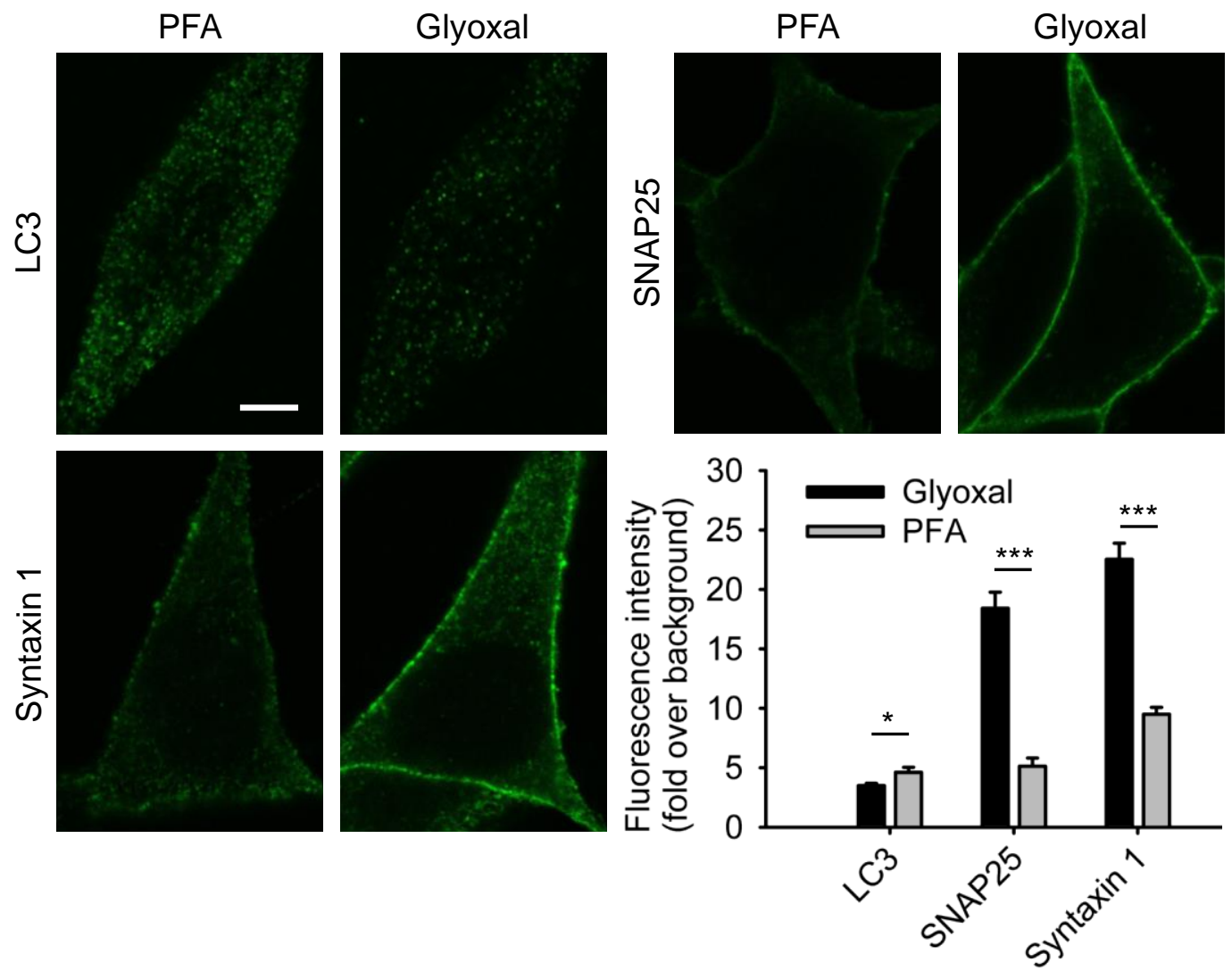

Figure 6 Comparison of immunostained AtT20 cells after fixation with either PFA or glyoxal.

AtT20 cells stained for the SNARE proteins syntaxin1 and SNAP25 and the autophagy marker LC3B, were compared with regard to the fluorescence intensity (fold over background) of the stainings. Quantification of the intensity shows that glyoxal fixation allows for significantly brighter stainings of the membrane SNARE proteins. LC3B staining is brighter in PFA fixed cells. $\mathrm{N}=9-20$ cells per condition. Scale bar $=5 \mu \mathrm{m} . * \mathrm{p}<0.05, * * * \mathrm{p}<0.001$ (two-sided Student's t-test).

The Hell laboratory (Department of NanoBiophotonics, Max Planck Institute for Biophysical Chemistry, Göttingen, Germany) used 3D STED microscopy to analyze the organization of several cytoskeletal proteins and of two membrane channels in axons and in growth cones of rat hippocampal cultured neurons (Fig. 7). Actin was labeled using phalloidin, while all other proteins were labeled by immunostaining with previously published antibodies. Phalloidin stainings were similar after PFA or glyoxal fixation in axons (Fig. 7A), but glyoxal revealed fine structures in growth cones that were not visible even after strong fixation with PFA and glutaraldehyde (Fig. 7D). Neurofilaments were brighter after glyoxal fixation (Fig. 7C), while another cytoskeletal element, Ankyrin G, was brighter for PFA fixations (Fig. 7A). The cytoskeletal protein $\beta$ II-spectrin was equally well stained in PFA or glyoxal fixation (Fig. 7C); the same was observed for voltage-gated sodium channels (Fig. 7B). The stainings for voltage-gated potassium channels were somewhat more regular for glyoxal fixation (Fig. 7D). Finally, the staining of tubulin was marginally less bright for glyoxal fixation when compared with a protocol optimized for tubulin stainings (fixation with PFA and glutaraldehyde) and dynamic microtubles in the growth cones were better preserved with that fixation (Fig. 7D). 
A

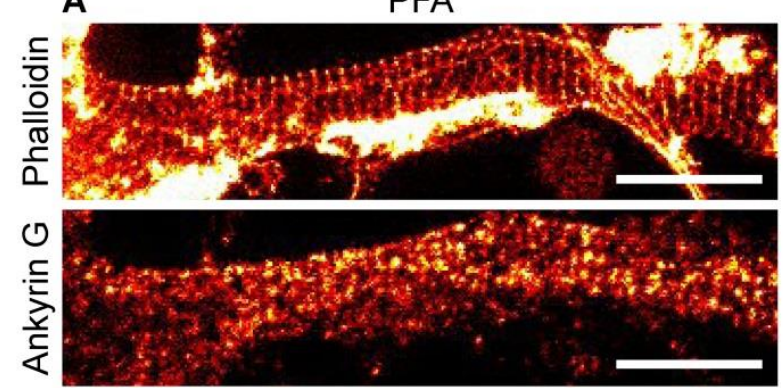

B
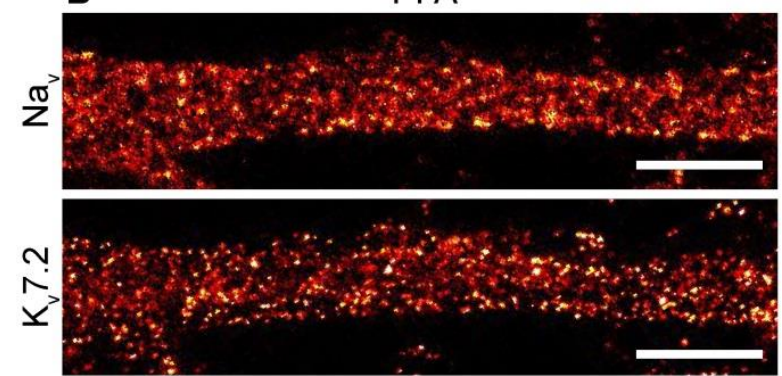

C
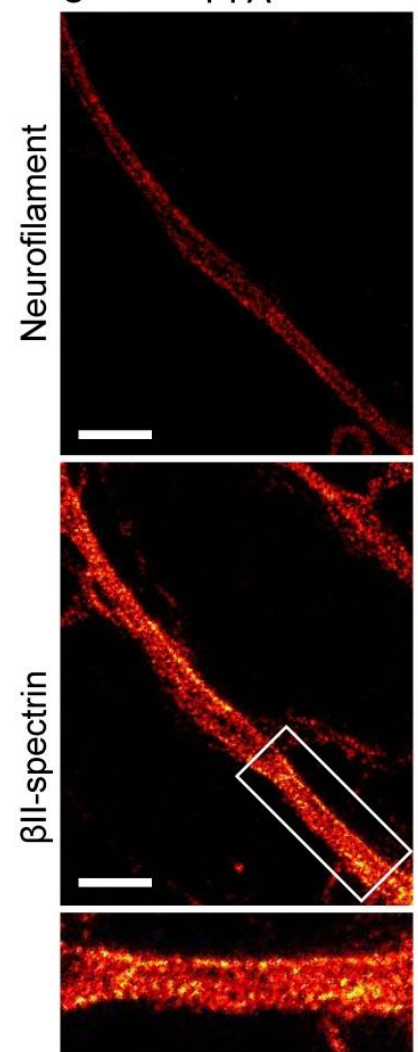

Glyoxal
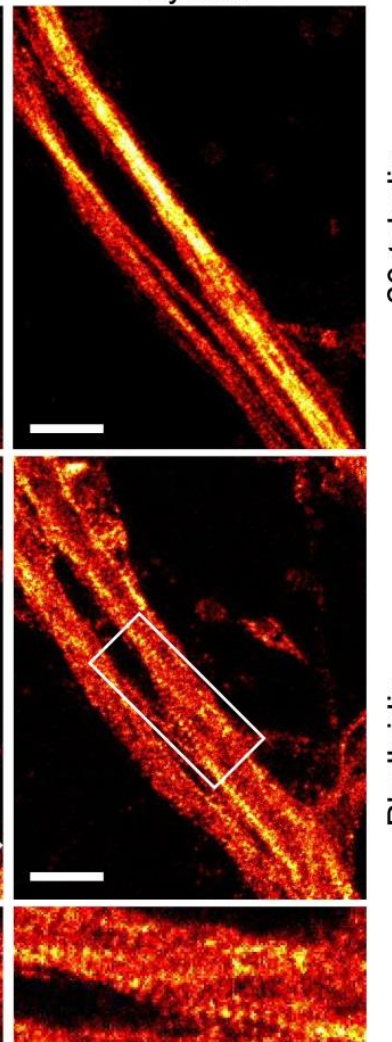
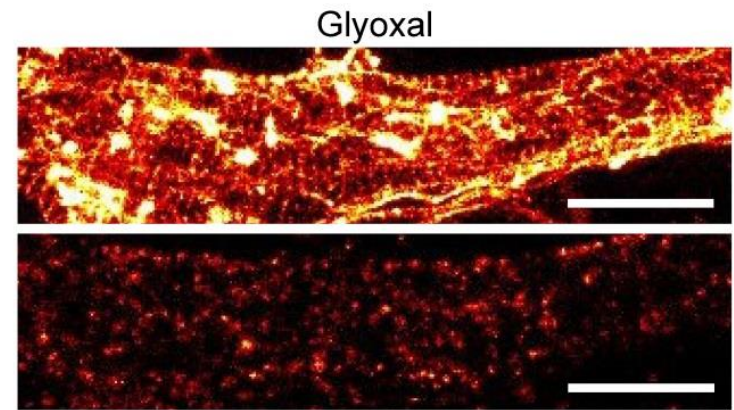

Glyoxal
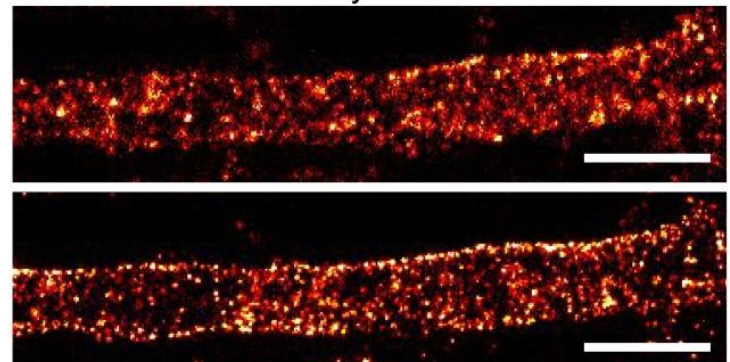

D PFA + Glut
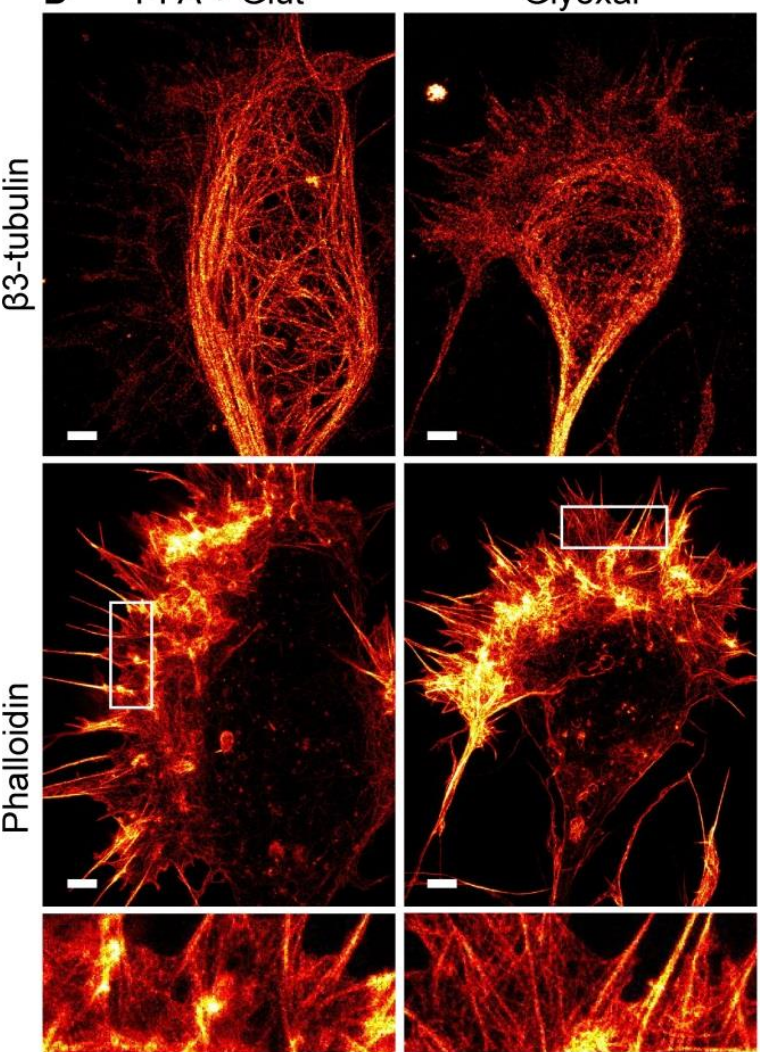

Figure 7 Comparison of immunostained primary hippocampal neurons in STED resolution.

A Primary hippocampal neurons were stained for Actin and Ankyrin G. A comparison between PFAand glyoxal-fixed samples shows that actin staining with phalloidin works as least as well in both, showing the prominent actin rings. Ankyrin $\mathrm{G}$ staining is brighter in PFA-fixed cells.

B Primary hippocampal neurons were stained for pan- $\mathrm{Na}_{\mathrm{v}}$ and $\mathrm{K}_{\mathrm{v}} 7.2$. Both stainings seem to work at least as well for glyoxal fixed neurons as for PFA fixed neurons. Staining of K-channels shows a slightly more regular pattern in glyoxal fixed neurons.

$C$ Primary hippocampal neurons were stained for Neurofilament $L$ and betall spectrin. While the spectrin staining seems to be equally well in both fixation conditions, neurofilament staining is brighter in glyoxal fixed cells. 
D Growth cones of hippocampal neurons were stained for actin and $\beta I I I-t u b u l i n$ after either glyoxal or PFA + glutaraldehyde fixation. The latter is a standard fixation used for the co-labeling of tubulin and actin, and is a stronger fixation than normal PFA fixation, which is incompatible with many organelle immunostainings (unlike glyoxal fixation). The filopodia and lamellipodia of the growth cones seem to be well stained for the samples fixed with glyoxal, whereas the samples fixed with PFA and glutaraldehyde seem to have lost some of the finer actin structures. Tubulin seems to be a bit better stained in samples fixed with PFA and glutaraldehyde. Scale bars $=1 \mu \mathrm{m}$.

The Lauterbach team (Max Planck Institute for Brain Research, Frankfurt am Main, Germany) focused on sepia fin immunostainings, testing the organization of FMRFamide in axons. PFA fixation resulted in poorer morphology, with fragmented axons, while glyoxal fixation revealed axons that appeared physiologically normal (Figure 8).

\section{Sepia Fin FMRFamide}

PFA

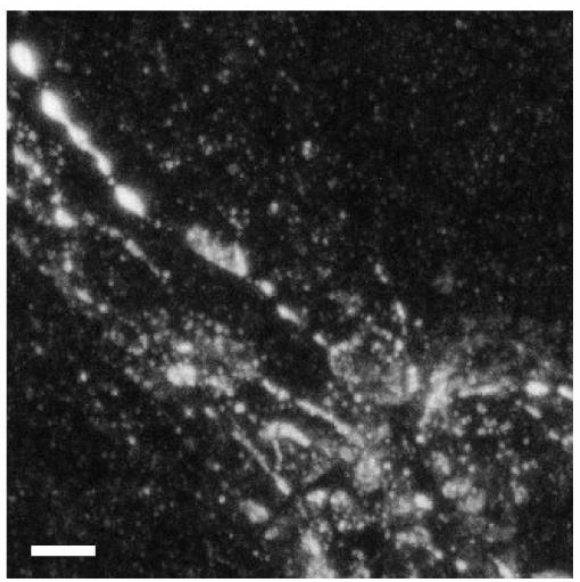

Glyoxal

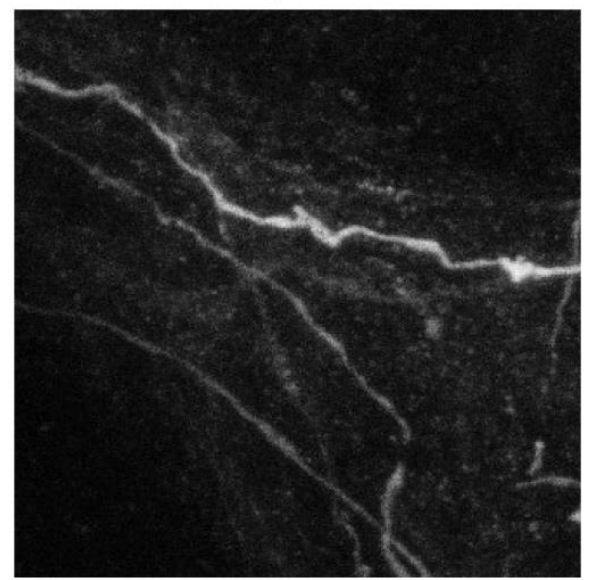

Figure $\mathbf{8}$ Comparison of immunostained sepia fin after fixation with either PFA or glyoxal.

Sepia fin was fixed with the respective fixative and stained for the neuropeptide FMRFamide. A clear change in morphology can be observed between samples fixed with PFA and samples fixed with glyoxal. The former appear broken and swollen, while the glyoxal-fixed ones appear complete. The effect is presumably due to the different speed of penetration into tissue and/or fixation. Scale bar = $5 \mu \mathrm{m}$.

The Lehnart laboratory (Heart Research Center, Department of Cardiology \& Pulmonology, University Medical Center Göttingen, Germany) analyzed the trafficking and signal domain scaffolding protein caveolin-3 and the ryanodine receptor (of the sarcoendoplasmic reticulum) in ventricular myocytes isolated from mouse hearts, using STED microscopy. The immunostaining intensity of both of these proteins was significantly higher for glyoxal fixation (Fig. 9). Of note, the morphology remained similar, as determined by 3D STED microscopy. 

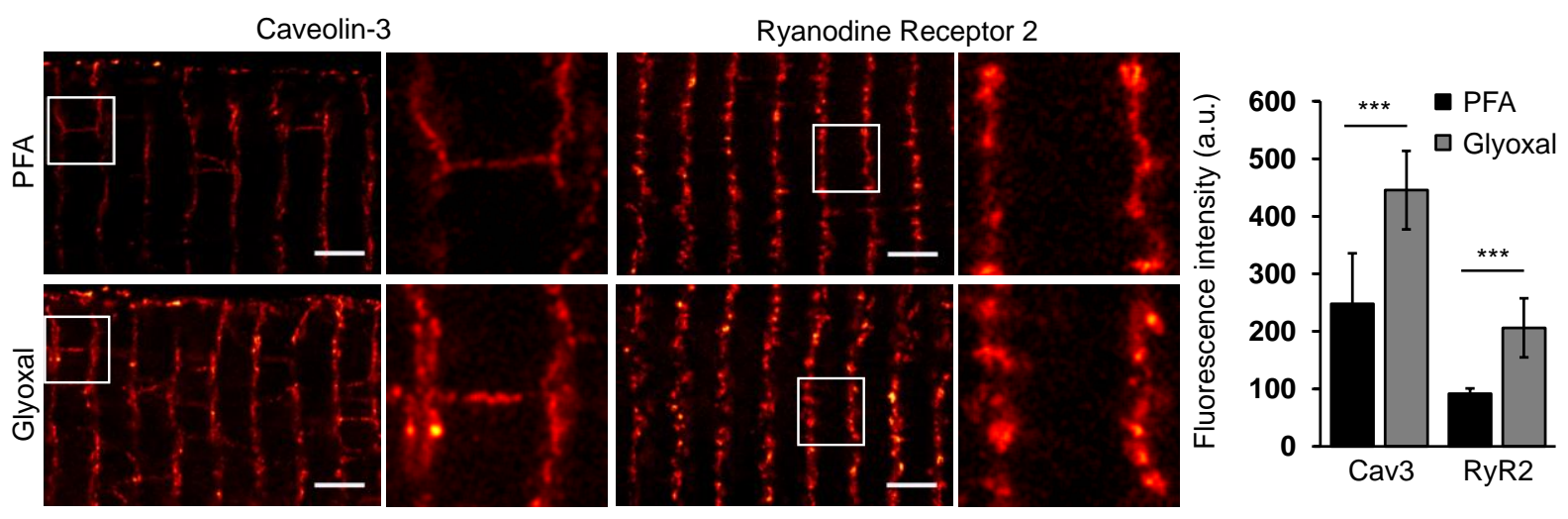

Figure 9 Comparison of immunostained ventricular myocytes fixed 10 min with either PFA or glyoxal.

Freshly isolated murine ventricular myocytes were either fixed with 4\% PFA or 3\% glyoxal and immunostained for caveolin-3 or ryanodine receptor type 2. Quantification of the fluorescence intensity of the stainings show significantly brighter stainings for glyoxal fixed myocytes. The graph shows mean values and error bars represent standard deviations. $N=10$ (RyR2) and 12 (Cav3) myocytes per condition. Scale bar $=2 \mu \mathrm{m} .{ }^{* * *} \mathrm{p}<0.001$ (Wilcoxon rank-sum test).

The Moser laboratory (Institute for Auditory Neuroscience and InnerEarLab, University Medical Center Göttingen; Auditory Neuroscience Group, Max-Planck-Institute for Experimental Medicine Göttingen, Germany) analyzed several proteins in the synapses formed between mouse cochlear inner hair cells and afferent spiral ganglion neurons (Fig. 10). No substantial differences were found for the active zone protein PSD95, for the trafficking protein otoferlin, and for the presynaptic voltage-gated calcium channel (CaV1.3). The signal intensities were substantially higher after glyoxal fixation for the presynaptic active zone protein $\mathrm{CtBP} 2$, the calcium buffer protein calretinin, and the postsynaptic active zone protein Homer 1. For none of the tested proteins was the signal found to be poorer in glyoxal in this preparation.
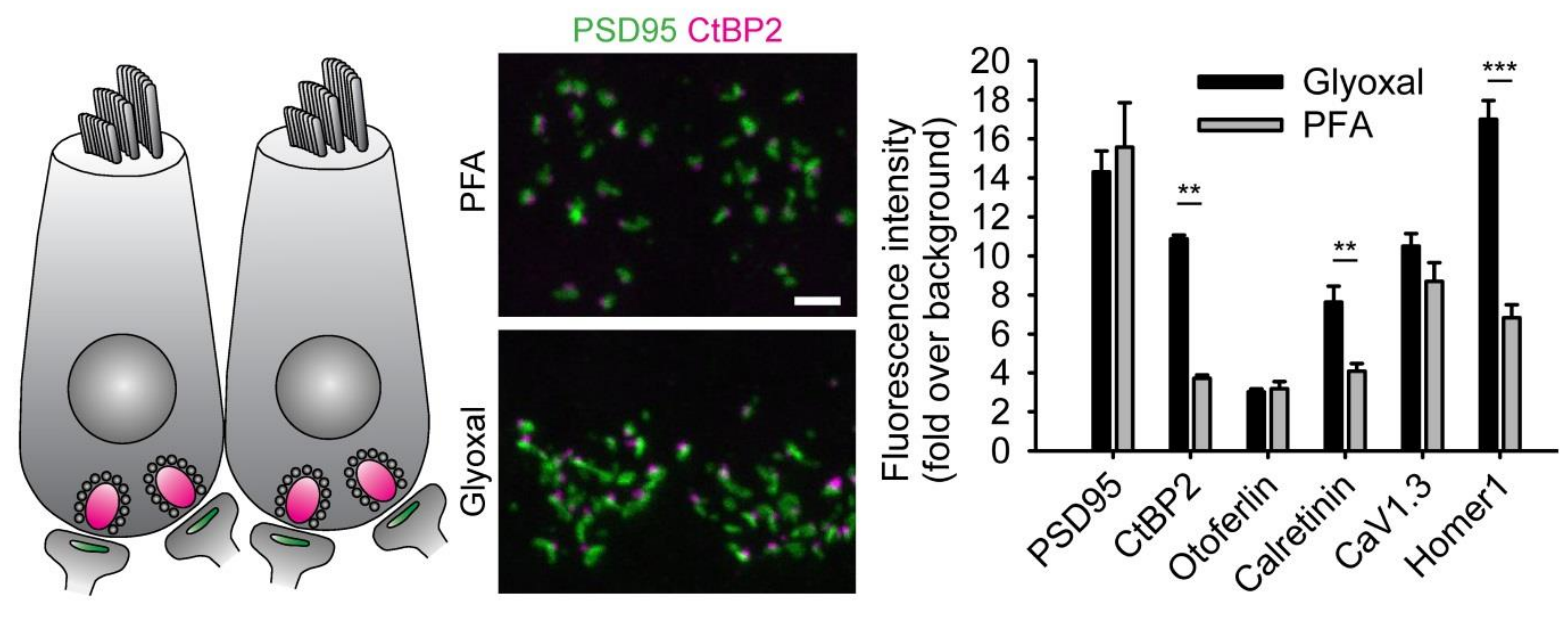

Figure 10 Comparison of immunostained mouse inner hair cells after fixation with either PFA or glyoxal.

Acutely dissected organs of corti were fixed in the respective fixative and immunostained for inner hair cell proteins and synaptic proteins. The quantification of fluorescence intensity for each staining shows a significant increase in signal to noise ratio for three target proteins (CtBP2, Calretinin and Homer1) fixed with glyoxal. None of the stained proteins shows a significant decrease in fluorescence 
after glyoxal fixation. Representative images show maximum intensity projections from z-stacks of inner hair cell ribbon synapses. $\mathrm{N}=5$ independent stainings from two animals per condition (PSD95, CtBP2, Otoferlin, Calretinin) and $10-15$ images per condition (CaV1.3 and Homer1). Scale bar = 2 $\mu \mathrm{m} .{ }^{* *} \mathrm{p}<0.01,{ }^{* * *} \mathrm{p}<0.001$ (two-sided Student's t-test for CtBP2, otoferlin, calretinin and Homer1, Wilcoxon rank-sum test for PSD95 and CaV1.3).

The Outeiro laboratory (Department of Experimental Neurodegeneration, Center for Nanoscale Microscopy and Molecular Physiology of the Brain, University Medical Center Göttingen; Max Planck Institute for Experimental Medicine, Göttingen, Germany) investigated the cytoskeletal protein vimentin, along with alpha-synuclein, a soluble protein whose propensity to aggregate is a potential cause for Parkinson's Disease (Fig. 11). Vimentin immunostainings were $\sim 20 \%$ brighter after PFA fixation. A stronger phenotype was observed for alpha-synuclein immunostainings, which were 2-fold brighter after glyoxal fixation. The Outeiro laboratory also tested a fluorescence tag, mOrange2, in the two fixation conditions, and found that $\sim 20 \%$ more fluorescence could be detected after glyoxal fixation.

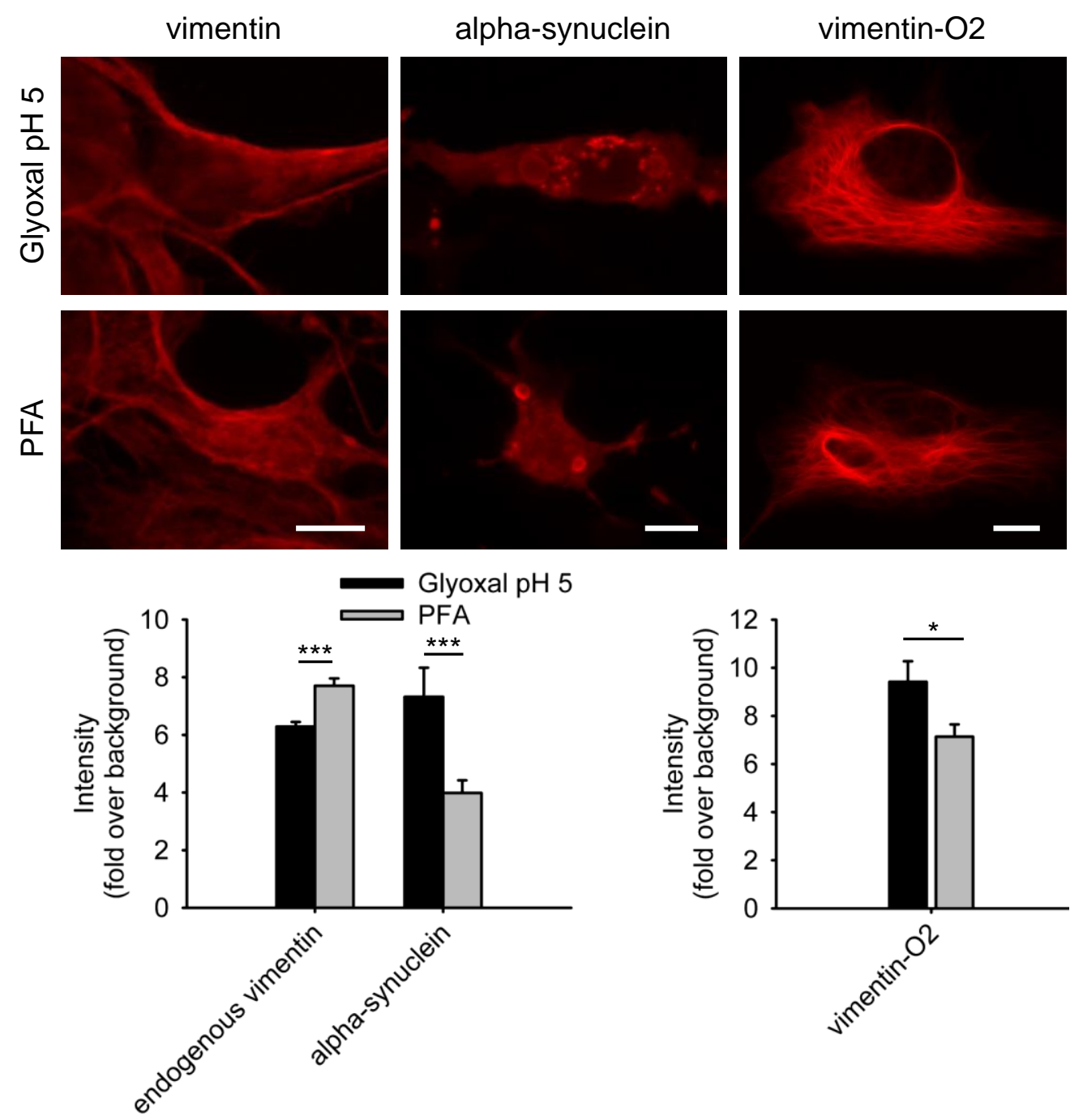

Figure 11 Comparison of stained vimentin and $\alpha$-synuclein in human neuroglioma cells after fixation with either PFA or glyoxal. 
Cells were fixed with PFA or glyoxal for 10 minutes, and were stained for endogenous vimentin, or for expressed alpha-synuclein. A quantification of the staining intensities indicates that glyoxal fixation allows for significantly brighter stainings for alpha-synuclein, but that PFA was superior for endogenous vimentin (leftmost graph). The fluorescence intensity of vimentin expressed with a mOrange2 tag was also analyzed after fixation with PFA or with glyoxal; the latter allowed more mOrange2 fluorescence to be detected (rightmost graph). $\mathrm{N}=29-81$ cell regions per condition. ${ }^{*} \mathrm{p}$ $<0.05,{ }^{* * *} \mathrm{p}<0.001$ (Wilcoxon rank-sum test). Scale bar: $10 \mu \mathrm{m}$.

The Rehling laboratory (Department of Cellular Biochemistry, University Medical Center Göttingen; Max Planck Institute for Biophysical Chemistry, Göttingen, Germany) analyzed several mitochondrial proteins in cell cultures, using conventional immunostainings, and found that the signals were better, albeit within the same range, for PFA fixations of TIM23, COA6, and NDUFA9. For ATP5B and for mitotracker stainings the situation was inversed, with glyoxal fixations providing substantially improved images (Fig. 12).

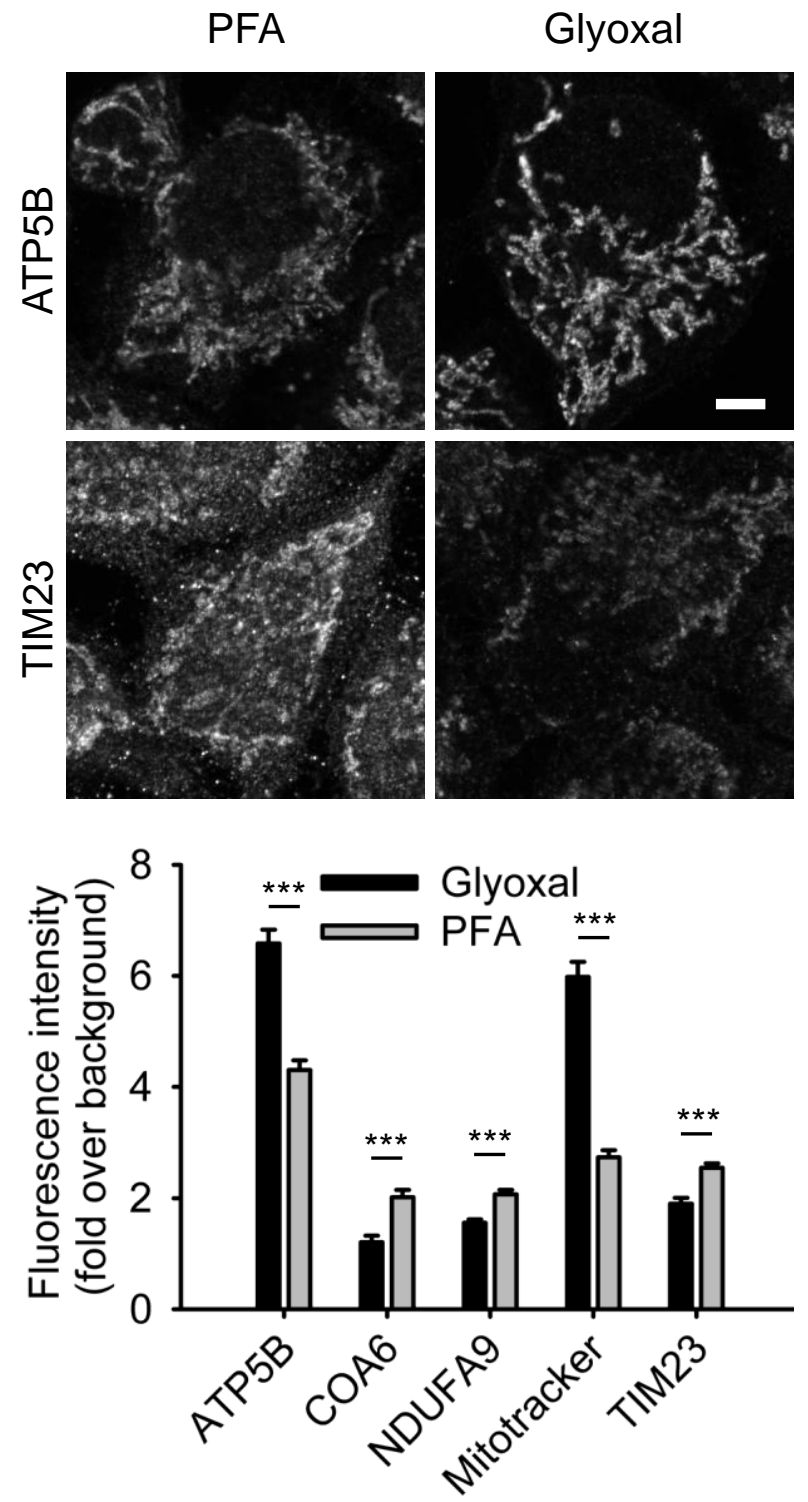

Figure 12 Comparison of immunostainings for mitochondrial proteins after fixation with either PFA or glyoxal. 
Cells were stained with MitoTracker Orange prior to fixation with the respective fixative and immunostained for the mitochondrial proteins ATP5B, COA6, NDUFA9 and TIM23. Quantification of the staining intensity shows a significant increase of fluorescence (signal over background) for 2 markers (ATP5B and Mitotracker) after fixation with glyoxal, whereas for the remaining 3 proteins immunostainings seem to be more efficient after fixation with PFA, albeit the differences are small. $\mathrm{N}$ $=18-128$ cells per condition. Scale bar $=5 \mu \mathrm{m} .{ }^{* * *} p<0.001$ (two-sided Student's t-test for ATP5B and NDUFA9, Wilcoxon rank-sum test for all other proteins).

The Schwappach laboratory (Department of Molecular Biology, University Medical Center Göttingen, Germany) also relied on cell cultures to analyze a large palette of proteins involved in several processes, from nuclear organization to mitochondria and to the secretory pathway. Seven proteins were similar for PFA and glyoxal fixations (with marginally dimmer staining for Sec61b, an endoplasmic reticulum protein, after glyoxal fixation). The immunostainings for the lysosomal marker LAMP1 and for ATPB, a component of the mitochondrial ATP synthase, were far poorer after glyoxal fixation than after PFA fixation. In contrast, eight target proteins provided brighter immunostainings after glyoxal fixation, with the largest differences seen for the Golgi marker GM130, and for the SNARE syntaxin 5 (Fig. 13).
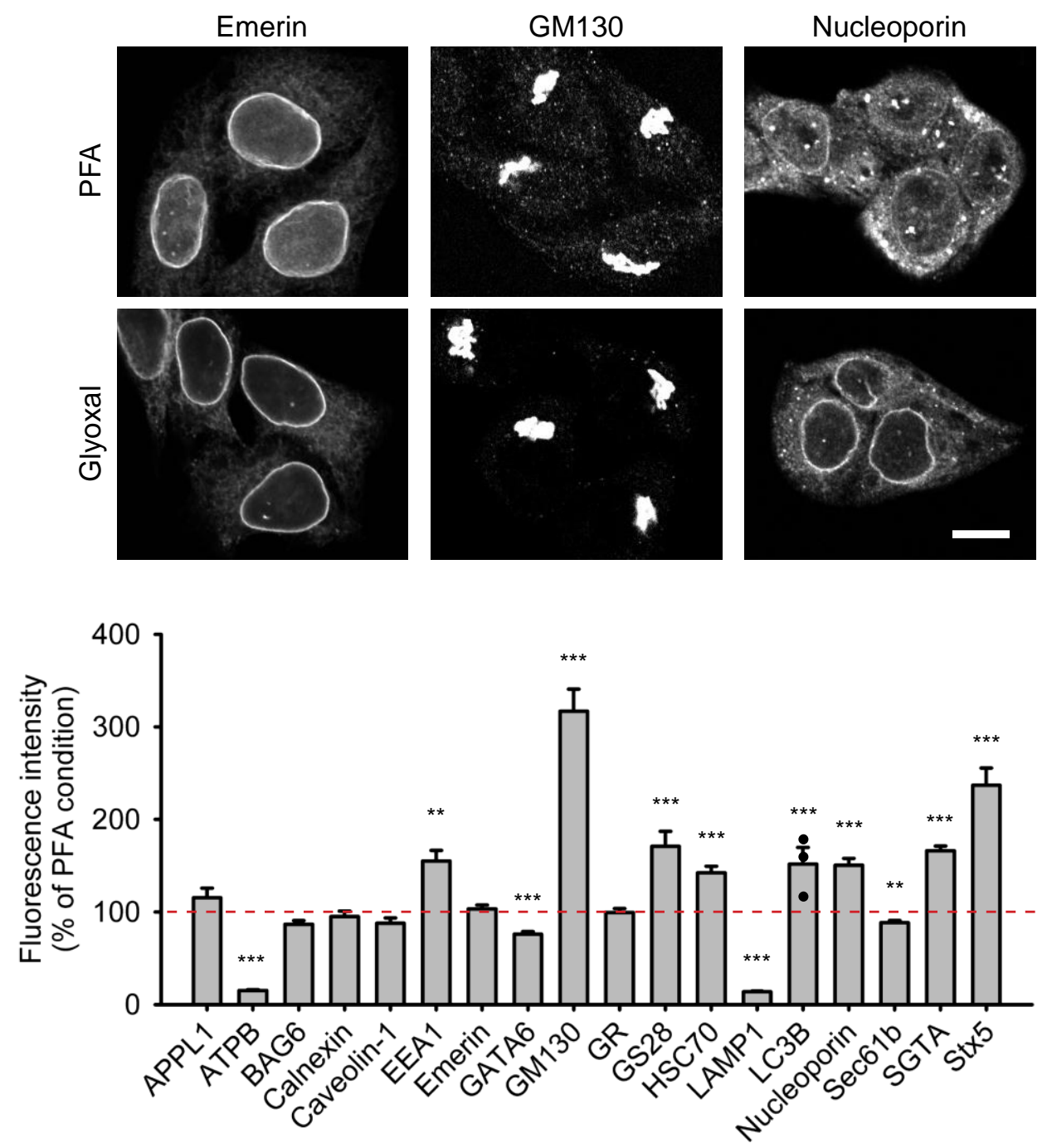

Figure 13 Comparison of immunostained HeLa cells after fixation with either PFA or glyoxal. 
The fluorescence intensity of a variety of proteins was compared between cells fixed with PFA or glyoxal. Quantification is shown as percentage of signal derived from PFA fixed cells. 8 out of the 18 target proteins which were stained show significantly brighter signal when fixed with glyoxal. Only 4 proteins show significantly reduced staining intensity. For LC3B the intensity of less than 5 cells were quantified, therefore single data points were plotted in addition to the bars. $\mathrm{N}=3-44$ cells per condition. Scale bar $=10 \mu \mathrm{m} .{ }^{* *} \mathrm{p}<0.01,{ }^{* * *} \mathrm{p}<0.001$ (Wilcoxon rank-sum test for APPL1, ATPB, BAG6, caveolin-1, GATA6 and HSC70, two-sided Student's t-test for all other proteins).

The Testa (Department of Applied Physics, KTH Royal Institute of Technology, Stockholm, Sweden) laboratory analyzed, using confocal microscopy, a membrane marker (the $\mathrm{Na}+/ \mathrm{K}+-$ ATPase), a mitochondria marker (Tom20), and an endoplasmic reticulum marker (overexpressed EGFP coupled to a KDEL signal) in primary cultured neurons or in cultured cells (Fig. 14). The $\mathrm{Na}+/ \mathrm{K}+-\mathrm{ATPase}$ was revealed correctly as a membrane protein only after glyoxal fixation, while it was found mostly in the nucleus after PFA fixation. Tom20 stainings were similar for the two fixations. EGFP-KDEL stainings had a poorer morphology after PFA fixation, with this protein apparently having spilled over from the endoplasmic reticulum during fixation in a quarter of all analyzed cells.

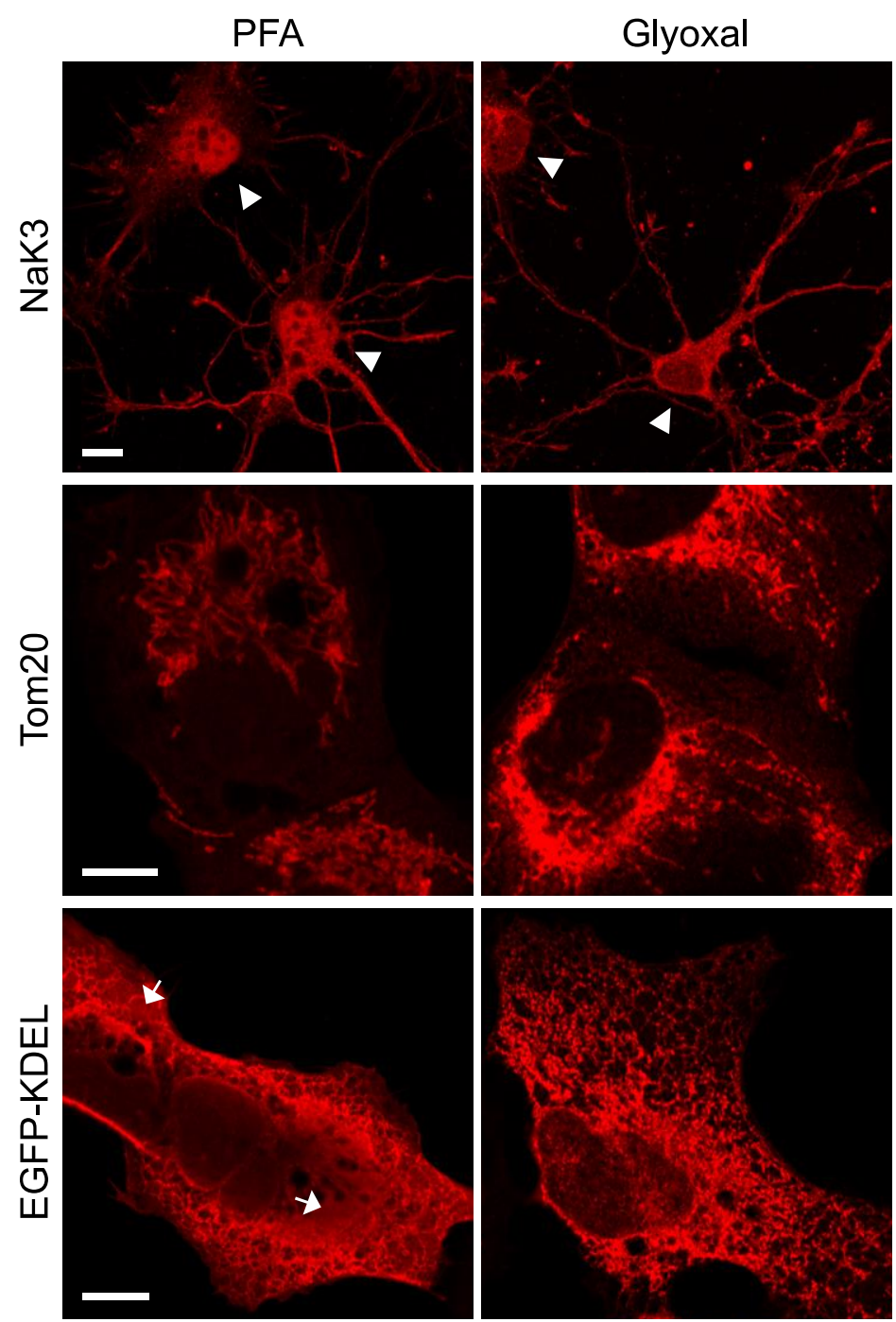

Figure 14 Comparison of immunostained U2OS cells and primary hippocampal neurons after either PFA or glyoxal fixation. 
Immunostaining of the $\mathrm{Na} / \mathrm{K}$ ATPase in primary hippocampal neurons shows a different distribution of the protein between PFA fixed and glyoxal fixed samples. While in PFA-fixed neurons the antibody falsely stains the nucleus as well as the cytoplasm ( $100 \%$ of the 82 cells we analyzed), in glyoxal-fixed neurons the nucleus is devoid of signal, and the membrane appears to be correctly labeled (arrowheads; $100 \%$ of the 60 cells we analyzed). U2OS cells were immunostained for mitochondria (Tom20) and ER (EGFP-KDEL). The fixation and/or staining of mitochondria seems to be comparable in glyoxal and in PFA fixed cells. The staining of the ER shows an improved signal to noise ratio. The signal appears de-localized from the ER for multiple PFA-stained cells ( $25 \%$ of 36 analyzed cells, see arrows), while this is rare for the glyoxal-stained cells (3.4\% of 58 analyzed cells). Scale bar $=10 \mu \mathrm{m}$.

Finally, the Zapiec group (Max Planck Research Unit for Neurogenetics, Frankfurt am Main, Germany) analyzed different proteins in the mouse olfactory epithelium and bulb, and found substantially stronger immunostainings after glyoxal fixation for the olfactory marker protein (OMP; Fig. 15A-B), for neuropilin-1 and neuropilin-2 (Fig. 15C-D), and for the vesicular glutamate transporter 2 (Fig. 15E-F). The same was observed for $\beta 3$-Tubulin immunostainings (Fig. 15A-B and E-F). 
4\% PFA in PBS

$3 \%$ glyoxal pH 4

mouse olfactory epithelium
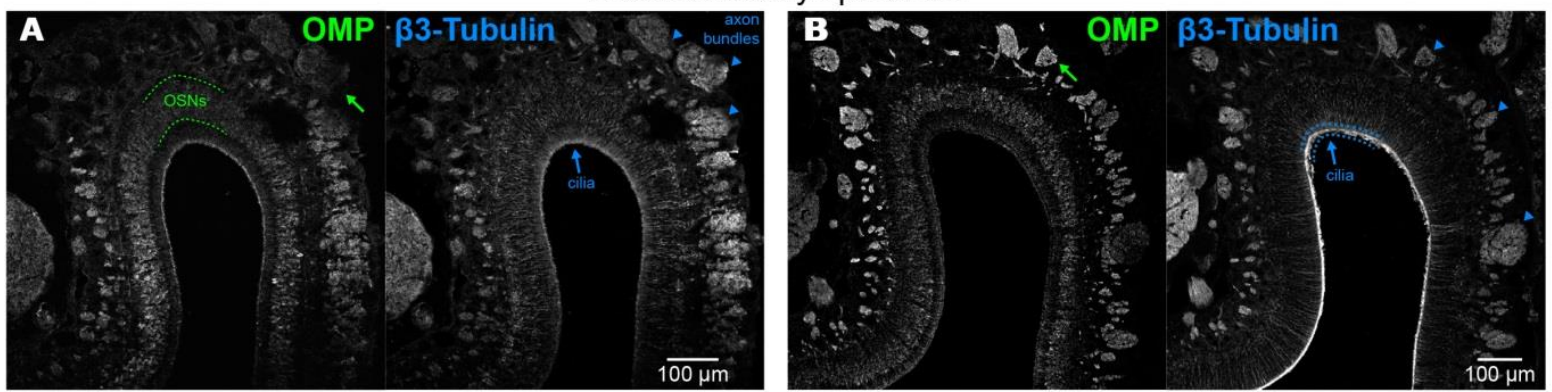

OSN axon bundles
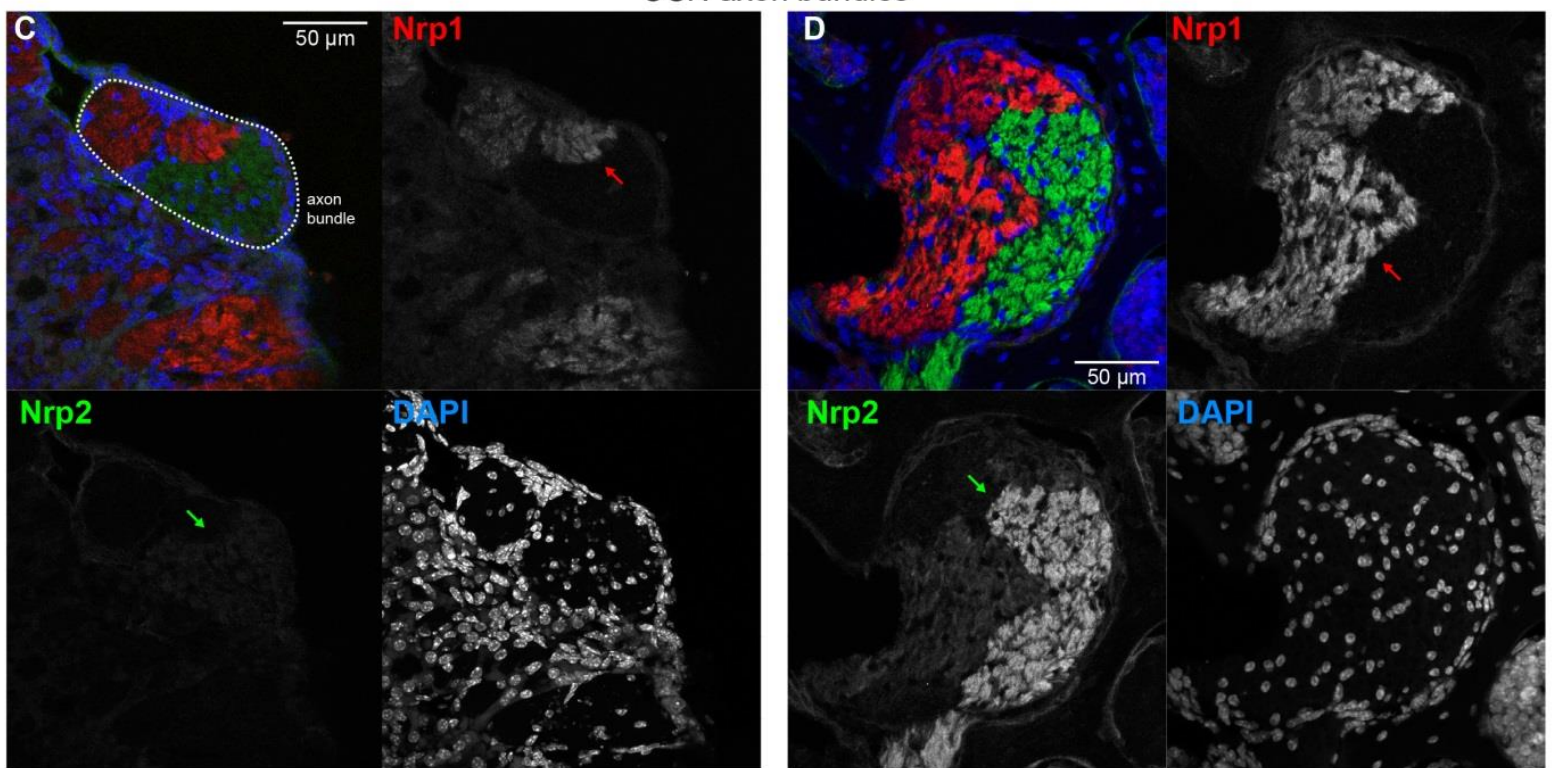

mouse olfactory bulb
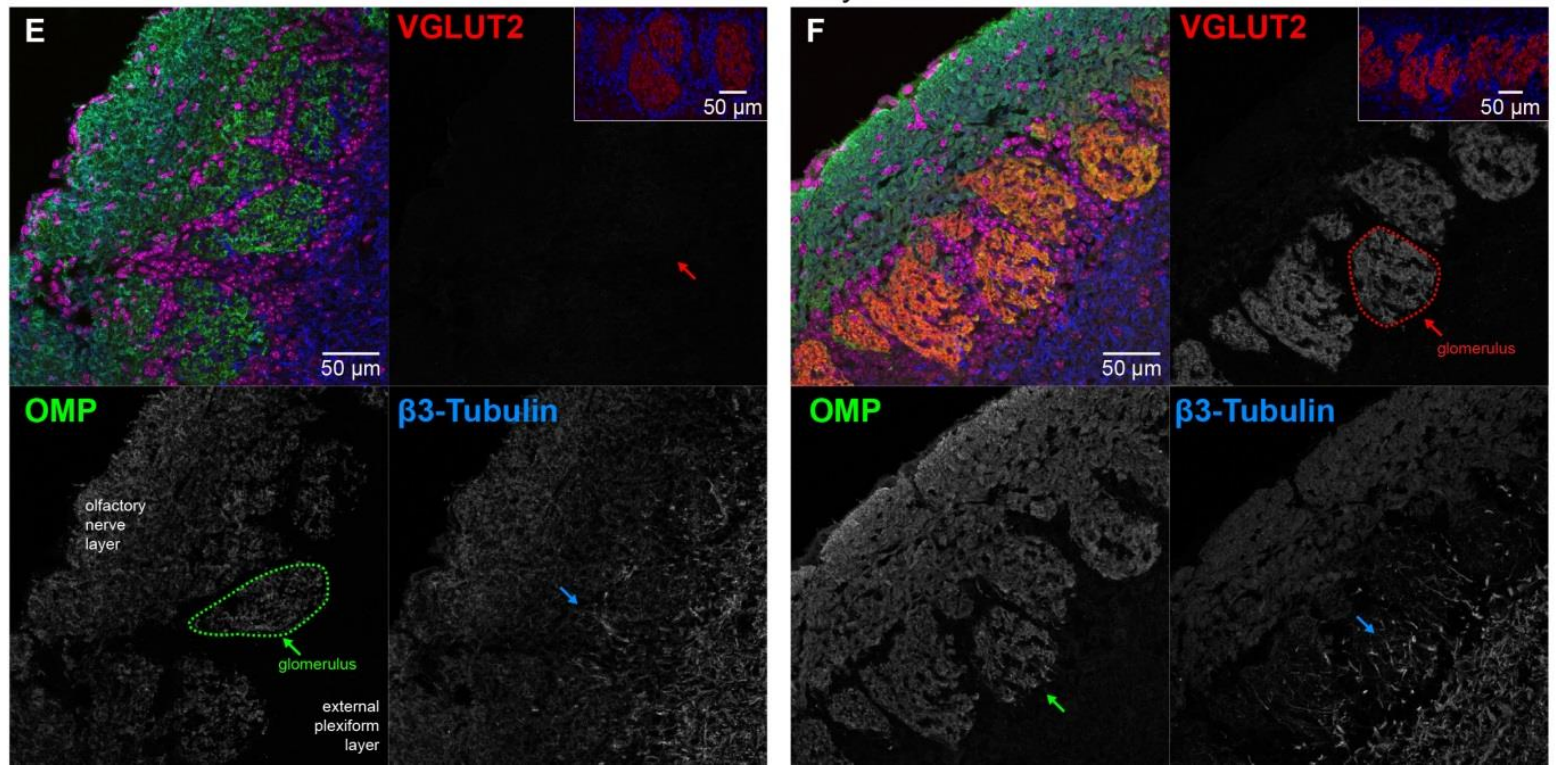

Figure 15 Comparison of mouse tissue staining following either PFA or glyoxal fixation.

A - B Confocal images showing staining of Olfactory Marker Protein (OMP) and $\beta 3$-tubulin along the dorsal aspect of the mouse olfactory epithelium. While sections from both types of fixative show OMP signal in the olfactory sensory neuron somata, their dendrites, and axons, the axon bundles (green arrow) located above the olfactory epithelium exemplify the clear signal-to-noise ratio benefits of glyoxal fixation versus that of the PFA-fixative. Immunostaining with the $\beta 3$-tubulin 
antibody stains the dendrites and axons (blue arrowheads) in both PFA and glyoxal-fixed tissue, but strong staining of the cilia (blue arrows) can only be observed in the glyoxal-fixed sections (B).

C - D Confocal images depicting bundles of axons belonging to olfactory sensory neurons on the path toward the olfactory bulb. Identities of the axons are in part defined by the neuropilin-1 (Nrp1) and neuropilin-2 (Nrp2) expression levels, visualized here with antibodies raised against the two proteins. While complementary expression of the two molecules can be seen in the PFA-fixed sections (C), the glyoxal-fixed sections (D) exhibit profoundly improved signal-to-noise ratios for Nrp-1 (red arrows), and in the case of $\mathrm{Nrp}-2$, also the segmentation of the axon bundle into varying levels of Nrp-2 (green arrows).

E - F Confocal images of olfactory sensory neuron axons coalescing into glomeruli where they synapse with dendrites of olfactory bulb neurons. The axons of olfactory sensory neurons can be readily visualized with OMP staining (green) in the superficial olfactory nerve layer and terminating in glomeruli located below (green arrows). While sections fixed with either PFA or glyoxal display adequate staining levels, the signal distribution of the PFA-fixed tissue appears more irregular (E), seemingly lacking the neurofilimentary morphology that appears preserved in the glyoxal-fixed sections (F). The glomeruli themselves are neuropil structures comprised primarily of olfactory sensory neurons forming synapses with dendrites of mitral/tufted cells as well as dendrites of periglomerular neurons. Immunostaining with Vesicular Glutamate Transporter 2 (VGLUT2) allows visualization of these structures and is easily seen in the glyoxal-fixed section (F), while it appears the antigen was masked by PFA fixation as no signal above background can be seen in the PFA-fixed panel (red arrows in E). Note that a different polyclonal antibody for VGLUT2 from the same provider does provide signal with PFA, albeit weaker versus Glyoxal (inset). Staining with $\beta 3$-tubulin touts the benefits of glyoxal both due to the signal improvements in the case of the mild staining in the axons of the olfactory nerve layer that is only visible in the glyoxal-fixed tissue, but also in preserving tissue morphology as demonstrated by the dendritic processes inside glomeruli (blue arrows) and in the external plexiform layer located below the glomeruli.

\subsection{Discussion}

We conclude that glyoxal fixation appears to be more efficient than PFA for many laboratories, in several countries. An overview of the results, indicating the different cellular targets analyzed, is presented in Fig. 16. Overall, 51 targets were better stained after glyoxal fixation, 12 targets were stained worse and 19 targets were stained equally well, which implies that glyoxal fixation seems to be generally superior to PFA. The strongest difference is seen for membrane proteins and for proteins of the Golgi apparatus. The organelle for which glyoxal is least successful is the mitochondrion. 


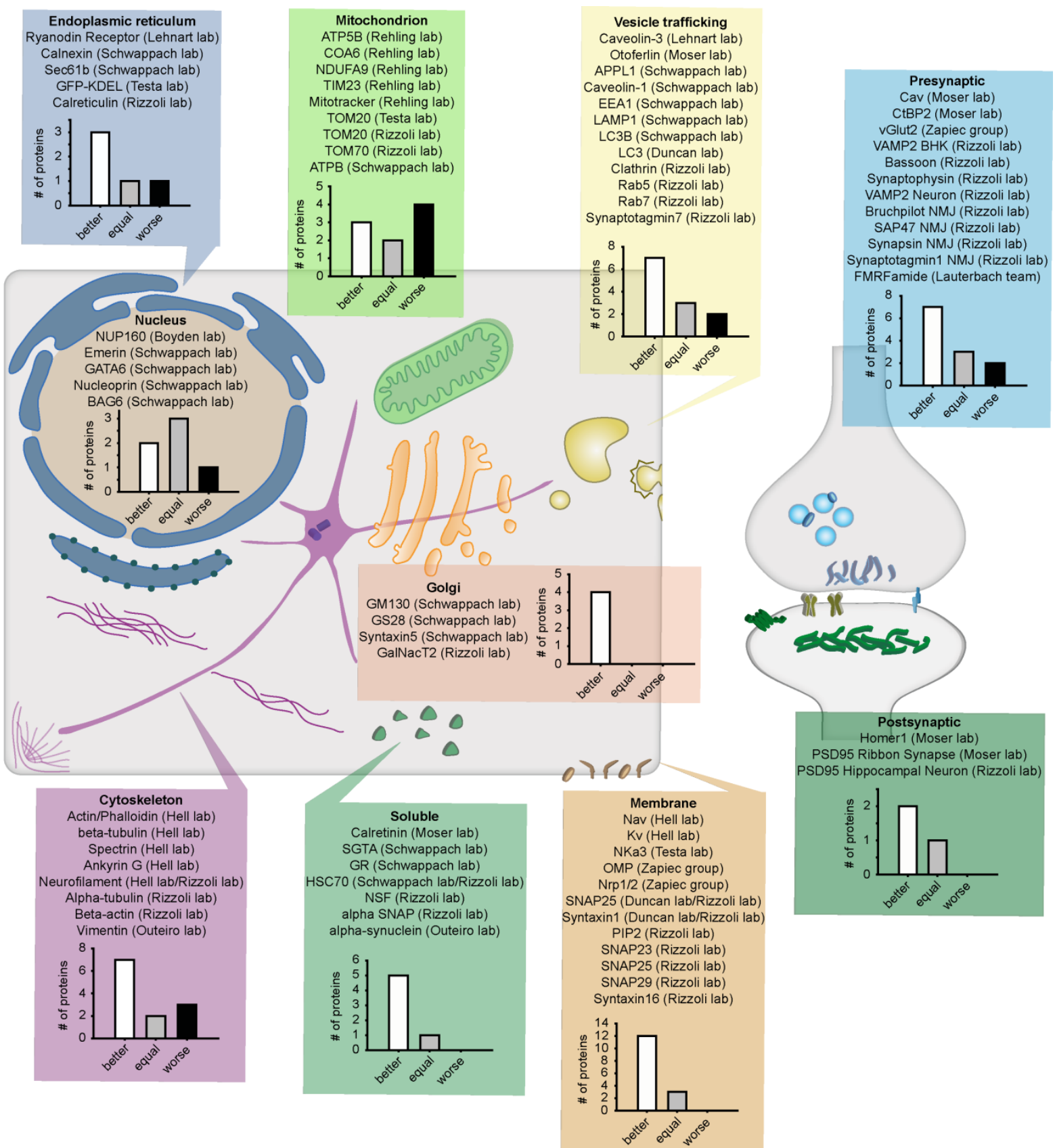

Figure 16 Overview of the results obtained from all immunostainings, in all of the laboratories testing glyoxal.

Various cellular targets, ranging from the nucleus to synapses of hippocampal neurons, were tested after fixation with either PFA or glyoxal by us and 11 additional laboratories. Overall, 51 targets were better stained after glyoxal fixation than after PFA fixation, 12 targets were stained worse, and 19 targets were equally well stained.

In principle, glyoxal could be combined with other fixatives, including glutaraldehyde, for an even stronger fixation. However, the behavior of aldehyde fixatives is exceedingly complex, leading to many side reactions (Migneault et al, 2004), which renders such an experiment difficult to reproduce. In a few trials, glutaraldehyde addition to glyoxal solutions actually caused poor morphology preservation, indicating that this may not be an optimal solution. Post-fixation with glutaraldehyde, for stronger and longer-lasting sample preservation, is 
nevertheless possible, as we observed in the electron microscopy experiments (Appendix Fig. S15; see also the respective Methods section).

Since glyoxal is substantially less harmful by inhalation than PFA (Wicks \& Suntzeff, 1943), we suggest that it should replace PFA for many applications. Comparative testing will still be needed for every antibody before settling on a fixation procedure. Nonetheless, we found that glyoxal typically provides immunostainings of better quality than PFA. In the few cases in which PFA provided brighter images, the glyoxal images were nevertheless still usable, revealing structures that appeared biologically accurate, with the clear exception of the lysosome marker LAMP1 and of the mitochondrial ATP synthase (Fig. 13). The opposite situation, however, was far more often encountered, especially for the membrane proteins such as the $\mathrm{Na}+/ \mathrm{K}+-$ ATPase (Fig. 14), the SNAREs SNAP25 and Syntaxin1 (Fig. 6), or multiple proteins of the mouse olfactory epithelium (Fig. 15)

While an extensive discussion of why this may be the case is beyond the purpose of this work, and would require an in-depth analysis of the fixation chemistry of both PFA and glyoxal, it is probable that the appearance of uniformly distributed small spots in PFA-fixed samples (Fig. 4 and Appendix Discussion) is due to insufficient cross-linking of proteins. The appearance of such spots has been a concern since the initial applications of superresolution microscopy (see for example Lang \& Rizzoli, 2010, which mostly revealed structures of $~ 70-100 \mathrm{~nm}$ in size. The fact that PFA only fixes about $60 \%$ of the proteins (Fig. 3) implies that a large fraction of the proteins is still mobile, can change its distribution during immunostaining, and may be even lost from the samples. We assume that the faster and stronger fixation induced by glyoxal (Fig. 1,2) plays a central role in improving the quality of the immunostainings, by maintaining the proteins in their organelle locations.

We conclude that this feature, the stronger and more accurate fixation, makes glyoxal a good candidate for the fixative of choice in immunostainings. In our opinion, glyoxal should still be preferred even for targets for which the two fixatives work equally well, because PFA presents substantially more health hazards than glyoxal during normal, routine laboratory work (Wicks \& Suntzeff, 1943).

\subsection{Materials and Methods}

\section{Paraformaldehyde (PFA) and glyoxal preparation}

For all experiments a $4 \% \mathrm{w} / \mathrm{v}$ paraformaldehyde (Sigma Aldrich \#P6148) solution and a $3 \%$ $\mathrm{v} / \mathrm{v}$ glyoxal (Sigma Aldrich \#128465) solution were used. Paraformaldehyde was dissolved in

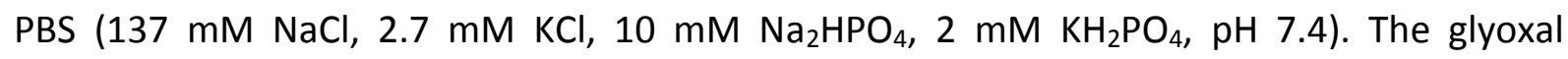
solution was prepared according to the following protocol: 
For $\sim 4 \mathrm{ml}$ glyoxal solution mix:

\begin{tabular}{|c|c|}
\hline- & $2.835 \mathrm{ml} \mathrm{dd} \mathrm{H}_{2} \mathrm{O}$ \\
\hline- & $0.789 \mathrm{ml}$ ethanol (absolute, for analysis) \\
\hline - & $0.313 \mathrm{ml}$ glyoxal (40 \% stock solution from Sigma Aldrich, \#128465) \\
\hline & $0.03 \mathrm{ml}$ acetic acid \\
\hline
\end{tabular}

Vortex the solution and bring the $\mathrm{pH}$ to 4 or 5 by adding drops of $1 \mathrm{M} \mathrm{NaOH}$ until respective $\mathrm{pH}$ is reached. Check $\mathrm{pH}$ with $\mathrm{pH}$ indicator paper. The solution should be kept cool and used within a few days, otherwise glyoxal might precipitate. If the stock solution shows precipitation, glyoxal can be redissolved by heating the solution to $\sim 50{ }^{\circ} \mathrm{C}$ (see also information provided by Sigma Aldrich).

Results obtained with glyoxal at $\mathrm{pH} 5$ are shown in all figures, unless noted otherwise (Appendix Fig. S2 shows data obtained from glyoxal $\mathrm{pH} 4$ ). For several control experiments (as noted in the figure legends), the same amount of ethanol was added to the PFA solution. The fixatives for the SDS-PAGE experiments (Fig. 3a, Appendix Fig. S7) were prepared so that the final amount of PFA and glyoxal (mixed with the cytosol samples) were $4 \%$ and $3 \%$, respectively. As a control for the SDS-PAGE experiments, $0.2 \%$ glutaraldehyde (AppliChem \#A3166) was added to a $4 \%$ PFA solution, as noted in the respective figure legend.

\section{Propidium iodide and FM 1-43 imaging}

Measuring cell penetration by the fixative (Fig. 1a, 1b; Appendix Fig. S1) was done using the dyes propidium iodide (Sigma Aldrich \#P4170) and FM 1-43 (Biotium \#70020). COS-7 fibroblast cells (obtained from the Leibniz Institute DSMZ - German Collection of Microorganisms and Cell Culture), plated on Poly-L-lysine (PLL)-coated coverslips and cultured under standard conditions, were washed briefly in pre-warmed cOS-7 cell Ringer (130 mM NaCl, $4 \mathrm{mM} \mathrm{KCl}, 5 \mathrm{mM} \mathrm{CaCl}$, 1 mM MgCl , 48 mM glucose, $10 \mathrm{mM} \mathrm{HEPES,} \mathrm{pH} \mathrm{7.4).}$ Afterwards the respective fixatives were added to the cells, containing either propidium iodide $(5 \mu \mathrm{M})$ or FM 1-43 (1.5 $\mu \mathrm{M})$. The cells were imaged for $60 \mathrm{~min}$ or $10 \mathrm{~min}$, respectively, using an inverted epifluorescence microscope (Nikon Eclipse Ti-E), as described in the Imaging section, below.

To determine the intensity of the propidium iodide stainings (Fig. 3b), COS-7 cells were fixed in the appropriate fixative for $30 \mathrm{~min}$ on ice, and for another $30 \mathrm{~min}$ at room temperature, followed by 20 min of quenching in $100 \mathrm{mM} \mathrm{NH}_{4} \mathrm{Cl}$ and $100 \mathrm{mM}$ glycine. After washing in PBS for $5 \mathrm{~min}$, the cells were incubated in $5 \mu \mathrm{M}$ propidium iodide in PBS, for $10 \mathrm{~min}$ at room temperature. After a $15 \mathrm{~min}$ wash-off in PBS, the cells were imaged using the same microscope as in the previous paragraph.

For the optimization of glyoxal fixation (Appendix Table 1) cultured primary hippocampal neurons were fixed for $30 \mathrm{~min}$ on ice and another $30 \mathrm{~min}$ at room temperature in the respective fixative, followed by $10 \mathrm{~min}$ quenching in $100 \mathrm{mM} \mathrm{NH}_{4} \mathrm{Cl}$. The neurons were washed 2 times briefly in PBS and imaged in a 1.5 $\mu \mathrm{M}$ FM 1-43 solution using an Olympus IX71 inverted epifluorescence microscope described below in the imaging section. 


\section{Fluorescence in situ hybridization (FISH)}

FISH (Fig. 3b) was performed using the QuantiGene ${ }^{\circledR}$ ViewRNA ISH Cell Assay kit (Affymetrix \#QVC0001), according to the protocol provided by Affymetrix. In short, cultured rat hippocampal neurons were fixed in one of the tested fixatives for $10 \mathrm{~min}$ on ice and for another $20 \mathrm{~min}$ at room temperature. After a washing step, the cells were incubated in the provided detergent solution, followed by probe hybridization for 3 hours at $40{ }^{\circ} \mathrm{C}$ (using standard probes for GAPDH, provided with the kit by the manufacturer). Afterwards the samples were washed in the provided wash buffer and signal amplification was done by incubating the samples in pre-amplifier and amplifier solution for $30 \mathrm{~min}$ each at $40^{\circ} \mathrm{C}$. Label hybridization was done as well for $30 \mathrm{~min}$ at $40{ }^{\circ} \mathrm{C}$ using $\mathrm{Cy} 5$ as dye. After washing in wash buffer and PBS, the samples were embedded in Mowiol and imaged using an inverted Nikon Eclispe Ti-E epifluorescence microscope.

\section{Transferrin, LysoTracker ${ }^{\circledR}$ and cholera toxin uptake assay}

Live imaging of transferrin (coupled to Alexa Fluor 594, Thermo Fisher \#T133433) and cholera toxin subunit B (coupled to Alexa Fluor 555, Thermo Fisher \#C34776) uptake during fixation (Appendix Fig. S4) was done in COS-7 and HeLa (obtained from the Leibniz Institute DSMZ - German Collection of Microorganisms and Cell Culture) cells. The cells, plated on PLL-coated coverslips, were incubated in $25 \mu \mathrm{g} / \mathrm{ml}$ transferrin or $1 \mu \mathrm{g} / \mathrm{ml}$ cholera toxin at $37^{\circ} \mathrm{C}$ for $10 \mathrm{~min}$. Afterwards, the cells were washed in pre-warmed COS-7 cell Ringer and were imaged. A concentrated solution of each fixative was added to the Ringer, so that the final concentration of fixative was $4 \%$ for PFA and $3 \%$ for glyoxal. The cells were imaged during the first $10 \mathrm{~min}$ of fixation using the inverted Nikon Eclispe Ti-E epifluorescence microscope.

The imaging of transferrin and LysoTracker uptake at different time points during fixation (Appendix Fig. S2 and S3) was done in HeLa and COS-7 cells. The cells were incubated in the respective fixative for $3 \mathrm{~min}, 5 \mathrm{~min}, 10 \mathrm{~min}, 15 \mathrm{~min}$ and $20 \mathrm{~min}$ at $37^{\circ} \mathrm{C}$ prior to the addition of $25 \mu \mathrm{g} / \mathrm{ml}$ transferrin Alexa594 or $50 \mathrm{nM}$ LysoTracker Red DND-99 (Thermo Fisher \#L7528). Each sample was incubated in the fixative and transferrin/LysoTracker for 20 more min. The cells were then washed with PBS and embedded in Mowiol. The samples were imaged with a confocal TCS SP5 microscope (Leica).

\section{Lipofectamine transfection of COS-7 cells, HeLa cells and BHK cells}

For the imaging of preservation of various GFP-tagged proteins and structures (Appendix Fig. S5 and S6), COS-7 fibroblasts or HeLa cells were transfected with a TOMM70 construct from S. cerevisiae, which was amplified by PCR and cloned into a pEGFP-N1 plasmid (Clonetech), as well as an EGFP-N1- $\alpha$-tubulin construct, a nEGFP-N1-SNAP25 construct, a mCherry-pCS2+GalNacT2 (which was a kind gift of Elena Taverna, MPI of Molecular Cell Biology and Genetics, Wieland Huttner group) construct and a mOrange2-N1-synaptopHluorin construct. The chimeric mOr2-SypHy indicator was created by substituting the superecliptic GFP from the original SypHy (Granseth et al, 2006) construct (purchased from Addgene, Cambridge, MA, USA) with the $\mathrm{pH}$-sensitive mOrange2 fluorescent protein (purchased from Addgene). 
One hour prior to transfection the cells were incubated in antibiotic-free medium. Lipofectamine $^{\circledR} 2000$ (Thermo Fisher \#11668) and the DNA (0.5 $\mu \mathrm{g}$ or $1 \mu \mathrm{g}$ per $18 \mathrm{~mm}$ cover slip) were incubated in OptiMEM (Thermo Fisher \#31985047) for $20 \mathrm{~min}$ and were subsequently added to the cells. The medium was changed back to normal culturing medium (DMEM containing fetal calf serum, glutamine, penicillin and streptomycin) the next day, and cells were imaged using an inverted Nikon Eclispe Ti-E epifluorescence microscope. The cells were imaged in COS-7 cell Ringer before fixation, and were imaged again after incubation in the different fixatives for $60 \mathrm{~min}$.

For transfection with the GFP-tagged target protein VAMP2 (Appendix Fig. S10), the following construct was used: pEGFP-N1-VAMP2 (backbone plasmid was purchased from Addgene). 2.5 hours prior to transfection the cells (BHK fibroblasts) were incubated in antibiotic-free medium. $1 \mu \mathrm{g}$ of DNA per $18 \mathrm{~mm}$ cover slip and Lipofectamine ${ }^{\circledR} 2000$ were incubated for $20 \mathrm{~min}$ in OptiMEM and afterwards added to the medium. Cells were incubated in the mixture overnight, and were immunostained the following day after transfection.

For SNAP-tag labeling (Appendix Fig. S9) HeLa cells were transfected with the following constructs: cytoplasmatic SNAP-tag (pSNAPf, purchased from New England Biolabs), $\alpha-$ synuclein-SNAP-tag, VAMP2-SNAP-tag, and transferrin receptor-SNAP-tag. The SNAP-tag fused to either the $\mathrm{N}$ - or the C-terminal of VAMP2 were created by PCR amplification of VAMP2 (Vreja et al, 2015) and insertion into the SNAP-tag plasmid by Gibson assembly (Gibson et al, 2009). The transferrin receptor (Opazo et al, 2012) and $\alpha$-synuclein (Lázaro et al, 2014) were amplified by PCR and inserted into the SNAP-tag plasmid by Gibson assembly. $1 \mu \mathrm{g}$ of DNA per coverslip was incubated for 20 min with Lipofectamine ${ }^{\circledR} 2000$ and $100 \mu \mathrm{g}$ of the mixture in OptiMEM was added to each coverslip. Cells were incubated overnight and labeling was done the following day, as described in the next section.

\section{SNAP-tag labeling}

Transfected HeLa cells were washed briefly in medium and then fixed with either PFA or glyoxal $\mathrm{pH} 5$ for $30 \mathrm{~min}$ on ice and another $30 \mathrm{~min}$ at room temperature. The cells were labeled with 0.3 $\mathrm{MM}$ SNAP-Cell TMR-Star (New England BioLabs \#S9105S) for $30 \mathrm{~min}$, and afterwards washed with PBS for $10 \mathrm{~min}$. TMR fluorescence was imaged at the Olympus IX71 inverted epifluorescence microscope.

\section{Immunocytochemistry of cultured primary hippocampal neurons}

Rat primary hippocampal neuron cultures (Fig. 4 and Appendix Fig. S12-14) were prepared as described before (Beaudoin et al, 2012; Opazo et al, 2010) and were cultured either under standard conditions, or in Banker arrangements, locally separated from the astrocyte feeder layer (as described in (Kaech \& Banker, 2006). The neurons, plated on Poly-L-lysine coated cover slips, were fixed in PFA (pH 7, pH 4/5 or with Et-OH) or glyoxal for 60 min and were subsequently quenched for $30 \mathrm{~min}$ in $100 \mathrm{mM} \mathrm{NH}_{4} \mathrm{Cl}$. The $\mathrm{pH}$ of the glyoxal solution used for fixation is presented in Table 1. For each antibody, we used the $\mathrm{pH}$ that provided a brighter immunostaining. Permeabilization and background epitope blocking were achieved by 
incubating the neurons for $15 \mathrm{~min}$ in blocking solution, containing $2.5 \%$ BSA and $0.1 \%$ Triton X-100 in PBS. The samples were incubated with primary antibodies diluted in blocking solution, for $60 \mathrm{~min}$ at room temperature. Table 2 presents the antibodies and their dilutions from $1 \mathrm{mg} / \mathrm{ml}$ stocks. After washing another $15 \mathrm{~min}$ in blocking solution, secondary antibodies were applied for $60 \mathrm{~min}$, at room temperature. Subsequent washing in high salt PBS (500 mM NaCl) and PBS was followed by embedding in Mowiol. The samples were imaged with a STED TCS SP5 microscope (Leica).

Table 1: The $\mathrm{pH}$ of glyoxal solution used for fixation of neuronal samples

\begin{tabular}{|c|c|}
\hline staining & $\mathbf{p H}$ \\
\hline \hline$\alpha / \beta$ SNAP & 4 \\
\hline$\alpha$-tubulin & 5 \\
\hline$\beta$-actin & 5 \\
\hline Bassoon & 4 \\
\hline Calreticulin & 5 \\
\hline Clathrin LC & 4 \\
\hline HSC70 & 4 \\
\hline Neurofilament L & 4 \\
\hline NSF & 4 \\
\hline PSD95 & 4 \\
\hline Rab5 & 4 \\
\hline Rab7 & 4 \\
\hline SNAP23 & 4 \\
\hline SNAP25 & 4 \\
\hline SNAP29 & 4 \\
\hline Syntaxin 1 & 5 \\
\hline Syntaxin 16 & 4 \\
\hline Synaptophysin & 5 \\
\hline Synaptotagmin 7 & 4 \\
\hline VAMP2 & 5 \\
\hline
\end{tabular}


Table 2: Antibodies used for the immunostaining of neuronal proteins.

\begin{tabular}{|c|c|c|c|}
\hline target protein & species & company & dilution \\
\hline \multicolumn{4}{|l|}{ primary antibodies } \\
\hline${ }^{*} \alpha / \beta$ SNAP & mouse & Reinhard Jahn & $1: 100$ \\
\hline$\alpha$-tubulin & rabbit & SySy (\#302203) & $1: 4000$ \\
\hline$\beta$-actin & mouse & Sigma Aldrich (A1978) & $1: 300$ \\
\hline Bassoon & mouse & Enzo Lifescience (\#SAP7F407) & $1: 100$ \\
\hline Calreticulin & rabbit & Cell Signaling (\#12238) & $1: 100$ \\
\hline Clathrin LC & mouse & SySy (\#113011) & $1: 1000$ \\
\hline HSC70 & mouse & Santa Cruz (\#sc-7298) & $1: 100$ \\
\hline Neurofilament L & rabbit & SySy (\#171002) & $1: 500$ \\
\hline NSF & rabbit & SySy (\#123002) & $1: 500$ \\
\hline PSD95 & mouse & Neuromap (\#75-028 (K28/43)) & $1: 200$ \\
\hline *Rab5 & mouse & Reinhard Jahn & $1: 100$ \\
\hline Rab7 & rabbit & Cell Signaling (\#9367) & $1: 100$ \\
\hline SNAP23 & rabbit & SySy (\#111202) & $1: 100$ \\
\hline SNAP25 & mouse & SySy (\#111002) & $1: 500$ \\
\hline SNAP29 & rabbit & SySy (\#111302) & $1: 500$ \\
\hline Syntaxin1 & mouse & SySy (\#110011) & $1: 300$ \\
\hline Syntaxin16 & rabbit & SySy (\#110162) & $1: 100$ \\
\hline *Synaptophysin & rabbit & Reinhard Jahn (G96) & $1: 1500$ \\
\hline Synaptotagmin7 & rabbit & SySy (\#105173) & $1: 100$ \\
\hline VAMP2 & mouse & SySy(\#104211) & $1: 1500$ \\
\hline \multicolumn{4}{|l|}{ secondary antibodies } \\
\hline anti-mouse IgG (Atto647N) & goat & Sigma Aldrich (\#50185) & $1: 150$ \\
\hline anti-rabbit IgG (Atto647N) & goat & Rockland (\#611-156-003) & $1: 500$ \\
\hline
\end{tabular}

Indicated antibodies $\left({ }^{*}\right)$ were kind gifts of Prof. Dr. Reinhard Jahn, Max Planck Institute for Biophysical Chemistry, Göttingen, Germany.

\section{Immunocytochemistry of HeLa and COS-7 cells}

HeLa cells that took up transferrin Alexa546 (see uptake assay described above) were immunostained for endosomes (EEA1; Appendix Fig. S11). The cells were fixed in the respective fixative for $30 \mathrm{~min}$ on ice and another $30 \mathrm{~min}$ at room temperature. Afterwards, they were quenched with $100 \mathrm{mM} \mathrm{NH}_{4} \mathrm{Cl}$ for $20 \mathrm{~min}$. Permeabilization and blocking was done for $15 \mathrm{~min}$ in $2.5 \% \mathrm{BSA}$ and $0.1 \%$ Triton X-100 in PBS. Subsequently, the cells were incubated in the primary antibody rabbit anti-EEA1 (Synaptic Systems \#237002), diluted 1:100 for $60 \mathrm{~min}$. After washing in blocking/permeabilization solution for $15 \mathrm{~min}$, the cells were incubated with the secondary antibodies for $60 \mathrm{~min}$. A donkey anti-rabbit antibody coupled to Atto647N (Rockland, diluted 1:500) were used. Subsequent washing in high salt PBS and normal PBS was followed by embedding in Mowiol, and the cells were imaged at the confocal TCS SP5 microscope (Leica).

Immunostaining of overexpressed GFP-tagged proteins (Appendix Fig. S10; see transfection described earlier) was done like descried above. Following primary antibodies were used: mouse anti-TOMM20 (Sigma Aldrich \#WH0009804M1), diluted 1:200, rabbit anti- $\alpha$-tubulin 
(Synaptic Systems \#302203), diluted 1:1000, mouse anti-VAMP2 (Synaptic Systems \#104211), diluted 1:200, mouse anti-TGN38 (BD Biosceince \#610898), diluted 1:100, mouse anti-SNAP25 (Synaptic Systems \# 111011), diluted 1:500.

Immunostainig of Phosphatidyl-Inositol-4,5-bisphosphat $\left(\mathrm{PIP}_{2}\right)$ was done as described above (Appendix Fig. S8). The primary antibody mouse anti-PIP 2 (Abcam \#ab11039), diluted 1:50, was used. As secondary antibody a donkey anti-mouse coupled to Cy2 was used in the dilution 1:100. The cells were imaged with the Olympus IX71 inverted epifluorescence microscope.

\section{Immunohistochemistry of Drosophila 3rd instar larvae neuromuscular junctions}

Drosophila melanogaster $3^{\text {rd }}$ instar larvae (Appendix Fig. S16) were dissected in standard Drosophila medium as described before (Jan \& Jan, 1976). The larvae were fixed for $30 \mathrm{~min}$ on ice, and for another $30 \mathrm{~min}$ at room temperature, followed by $30 \mathrm{~min}$ of quenching in 100 $\mathrm{mM} \mathrm{NH}_{4} \mathrm{Cl}$. Permeabilization and blocking were performed for $30 \mathrm{~min}$ in PBS containing 2.5 $\%$ BSA and $0.5 \%$ Triton X-100. Incubation in primary antibodies was done for 60 min at room temperature. The following antibodies were used: mouse anti-synaptotagmin 1 (3H2 2D7), diluted 1:50, mouse anti-synapsin (3C11), diluted 1:20, mouse anti-syntaxin (8C3), diluted 1:50, mouse anti-SAP47 (nc46), diluted 1:100, and mouse anti-bruchpilot (nc82), diluted 1:50. All antibodies were purchased from the Developmental Studies Hybridoma Bank at the University of lowa (DSHB). After 30 min of washing in the blocking solution (0.5 \% Triton X$100)$, the samples were incubated in a Cy3-labeled goat anti-mouse antibody (1:100, Dianova \#715-165-150) for $60 \mathrm{~min}$ at room temperature. Subsequently, larvae were washed in high salt PBS and PBS and embedded in Mowiol. The samples were then imaged using an Olympus inverted epifluorescence microscope.

\section{Immunohistochemistry of mouse inner hair cells}

Organs of Corti (Appendix Fig. S17) were dissected from P14 - P18 wild type mice in ice-cold HBSS (5.36 mM KCl, $141.7 \mathrm{mM} \mathrm{NaCl}, 10 \mathrm{mM}$ HEPES, $34 \mathrm{mM}$ L-glutamine, $6.9 \mathrm{mM}$ D-glucose, $1 \mathrm{mM} \mathrm{MgCl}_{2}, 0.5 \mathrm{mM} \mathrm{MgSO}_{4}, \mathrm{pH}$ 7.4). The inner hair cells were stimulated by incubating the tissue for $3 \mathrm{~min}$ in HBSS with high potassium $(65.36 \mathrm{mM} \mathrm{KCl})$ at $37^{\circ} \mathrm{C}$. Afterwards, the organs were fixed for $30 \mathrm{~min}$ on ice, and for another $30 \mathrm{~min}$ at room temperature. The subsequent quenching was performed for $30 \mathrm{~min}$ in $100 \mathrm{mM} \mathrm{NH}_{4} \mathrm{Cl}$ and $100 \mathrm{mM}$ glycine. The organs were then permeabilized and blocked for 30 min with PBS containing $0.5 \%$ Triton X-100 and $2.5 \%$ BSA. The primary antibodies mouse anti-otoferlin (Abcam \#ab53233), diluted 1:350, and rabbit anti-ribeye (Synaptic Systems \#192003), diluted 1:1500, were applied for $60 \mathrm{~min}$. After $30 \mathrm{~min}$ of washing, the organs were incubated in secondary antibodies for $60 \mathrm{~min}$. Atto647-labeled goat anti-mouse (1:250, Sigma Aldrich \#50185) and the Cy2-labeled goat anti-rabbit (1:100, Dianova \#111-225-144) secondary antibodies were used. Washing in high salt PBS and PBS was followed by embedding in melamine, as described previously (Revelo et al, 2014). Organs were then cut into $200 \mathrm{~nm}$ thin-sections using a Leica EM UC6 ultramicrotome. The sections were embedded in Mowiol and were imaged using a STED TCS SP5 microscope (Leica). 


\section{Immunohistochemistry of mouse levator auris longus neuromuscular junctions}

The levator auris longus muscle (Appendix Fig. S18) was dissected from adult mice in ice-cold mouse Ringer ( $5 \mathrm{mM} \mathrm{KCl}, 154 \mathrm{mM} \mathrm{NaCl}, 5 \mathrm{mM}$ HEPES, $11 \mathrm{mM}$ D-glucose, $1 \mathrm{mM} \mathrm{MgCl}$, $2 \mathrm{mM}$ $\mathrm{CaCl}_{2}, \mathrm{pH}$ 7.3). Prior to fixation, the acetylcholine receptors were stained by incubating the muscles in a 1:150 dilution of tetramethylrhodamine-labeled bungarotoxin (Sigma Aldrich \#T0195) for $15 \mathrm{~min}$. After washing the tissue for $15 \mathrm{~min}$ in mouse Ringer, it was fixed for 30 min on ice and another $30 \mathrm{~min}$ at room temperature. Quenching was performed in $100 \mathrm{mM}$ $\mathrm{NH}_{4} \mathrm{Cl}$ and $100 \mathrm{mM}$ glycine. The tissue was then permeabilized and blocked by incubating in PBS containing $0.5 \%$ Triton X-100 and $2.5 \%$ BSA for 30 min. Primary antibodies were applied for $60 \mathrm{~min}$. The following antibodies were used: mouse anti-bassoon (Enzo Lifescience \#SAP7F407), diluted 1:100, and rabbit anti-piccolo (Synaptic Systems \#142003), diluted 1:150. After $30 \mathrm{~min}$ of washing, secondary antibodies were applied for $60 \mathrm{~min}$ (Atto647labeled goat anti-mouse, Sigma Aldrich \#50185, diluted 1:150, and Cy2-labeled goat antirabbit, Dianova \#111-225-144, diluted 1:100). After 20 more min of washing in the blocking solution, $30 \mathrm{~min}$ in high salt PBS, and $20 \mathrm{~min}$ in PBS, the samples were embedded in 2,2'Thiodiethanol as described previously (Revelo \& Rizzoli, 2015) (TDE, Sigma Aldrich \#166782). The neuromuscular junctions were imaged using a STED TCS SP5 microscope (Leica).

\section{Imaging with an inverted epifluorescence Nikon Eclipse Ti-E microscope}

Experiments from Figure 1, 2, 3b, Appendix Figure S4, S5 and S6 were imaged using the Nikon inverted epifluorescence microscope. The microscope was equipped with an HBO 100W lamp and an IXON X3897 Andor Camera. For all samples a 60X Plan apochromat oil immersion objective (NA 1.4) was used (from Nikon). The filter sets and time course (if applicable) used for imaging are shown in Table 3. Images were obtained using the image acquisition software NiS-Elements AR (Nikon).

Table 3: Filter sets and time courses used for the Nikon Eclipse Ti-E microscope

\begin{tabular}{|c|c|c|c|c|}
\hline Figure panel & excitation filter & emission filter & dicroic mirror & time course \\
\hline \hline $1 a$ & Cy3: $545 / 25 \mathrm{~nm}$ & $605 / 70 \mathrm{~nm}$ & $565 \mathrm{~nm}$ & $\begin{array}{c}60 \mathrm{~min} \text {, every 5 } \\
\text { min }\end{array}$ \\
\hline $1 \mathrm{~b}$ & EGFP: $470 / 40 \mathrm{~nm}$ & $525 / 50 \mathrm{~nm}$ & $495 \mathrm{~nm}$ & $\begin{array}{c}10 \text { min, every 30 } \\
\text { seconds }\end{array}$ \\
\hline 2 & DIC & DIC & DIC & $\begin{array}{c}60 \text { min, every 5 } \\
\text { min }\end{array}$ \\
\hline $3 b$ & Cy3: $545 / 25$ & $605 / 70 \mathrm{~nm}$ & $565 \mathrm{~nm}$ & - \\
\hline $3 b$ & Cy5: $620 / 60 \mathrm{~nm}$ & $700 / 75 \mathrm{~nm}$ & $660 \mathrm{~nm}$ & - \\
\hline Appendix Fig. S5 & EGFP: $470 / 40 \mathrm{~nm}$ & $525 / 50 \mathrm{~nm}$ & $495 \mathrm{~nm}$ & - \\
\hline $\begin{array}{c}\text { Appendix Fig. S6 } \\
\text { (additional GFP) }\end{array}$ & EGFP: $470 / 40 \mathrm{~nm}$ & $525 / 50 \mathrm{~nm}$ & $495 \mathrm{~nm}$ & $\begin{array}{c}565 \mathrm{~nm} \\
\text { Appendix Fig. S4 }\end{array}$ \\
\hline & $\begin{array}{c}\text { Cy3: } 545 / 25 \mathrm{~nm} \\
\text { (cholera toxin) } \\
\text { (transferrin) }\end{array}$ & $\begin{array}{c}605 / 70 \mathrm{~nm} \\
\text { (cholera toxin) } \\
624 / 40 \mathrm{~nm} \\
\text { (transferrin) }\end{array}$ & $\begin{array}{c}\text { (cholera toxin) } \\
593 \mathrm{~nm} \\
\text { (transferrin) }\end{array}$ & $\begin{array}{c}10 \mathrm{~min} \text {, every 60 } \\
\text { seconds }\end{array}$ \\
\hline
\end{tabular}




\section{Imaging with a STED/confocal TCS SP5 microscope (Leica)}

The immunostained rat hippocampal neurons (Fig. 4, Appendix Fig. S12, S13, S14), mouse inner hair cells (Appendix Fig. S17) and neuromuscular junctions (Appendix Fig. S18), as well as the transferrin and LysoTracker uptake (Appendix Fig. S2, S3 and S11) and the immunostained GFP-tagged proteins (Appendix Fig. S10) were imaged using a pulsed STED microscope, built on the basis of the TCS SP5 confocal microscope (Leica). The microscope was equipped with a pulsed diode laser (18 mW, $80 \mathrm{MHz}, 640 \mathrm{~nm}$ emission, PicoQuant) for excitation of the STED dye, and with a pulsed infrared Titanium:Sapphire (Ti:Sa) tunable laser (1W, 80 MHz, 720 - 1000 nm, Mai Tai Broadband; Spectra-Physics) for depletion set at a wavelength of $750 \mathrm{~nm}$. For confocal imaging an Argon laser (488 nm) and HeNe laser lines (543 nm, $594 \mathrm{~nm}, 633 \mathrm{~nm}$ ) were used for excitation. Detection was achieved by ultrasensitive avalanche photodiodes and high sensitivity, low noise PMTs (Leica). All samples were imaged using a 100X HCX PL APO oil immersion STED objective (NA 1.4). Images were acquired using the Leica LAS AF imaging software, with a pixel size of $20 \times 20 \mathrm{~nm}, 30 \times 30 \mathrm{~nm}$ or $60 \times 60 \mathrm{~nm}$ and a scanning speed of $1000 \mathrm{~Hz}$.

\section{Imaging with an inverted epifluorescence Olympus IX 71 microscope}

The Drosophila larvae neuromuscular junctions (Appendix Fig. S16), the transfected and immunostained BHK (obtained from the Max-Planck-Institute for biophysical chemistry Göttingen, Reinhard Jahn) cells (VAMP2 expression in Appendix Fig. S10), the FM 1-43 stained neurons (Appendix Table 1), the COS-7 cells, stained for $\mathrm{PIP}_{2}$ (Appendix Fig. S8), and the SNAP-tag labeled HeLa cells (Appendix Fig. S9) were imaged using an Olympus IX 71 epifluorescence microscope, equipped with a $100 \mathrm{~W}$ mercury lamp and a F-View II CCD camera (Soft Imaging Systems $\mathrm{GmbH}$ ). The Drosophila NMJs and $\mathrm{PIP}_{2}$ stained COS cells were imaged using a 100X TIRFM oil immersion objective (NA 1.45), from Olympus. The BHK cells and the SNAP-tag labeled HeLa cells were imaged using the 40X UPlan FLN air objective (NA 0.75) from Olympus. The hippocampal neurons were imaged using a 60X UPlanApo oil immersion objective (NA 1.35) from Olympus. Filter sets used for imaging can be found in Table 4. Image acquisition was performed using the Olympus Cell^P software.

Table 4: Filter sets used for the Olympus IX 71 epifluorescence microscope

\begin{tabular}{|c|c|c|}
\hline filter & excitation & emission \\
\hline \hline FITC & 494 & 518 \\
\hline RFP & 561 & 585 \\
\hline Cy5 & 625 & 670 \\
\hline
\end{tabular}

\section{SDS-PAGE of fixed rat brain cytoplasm}

Rat brain cytosol (Fig. 3a and Appendix Fig. S7) was prepared by homogenization of adult rat brains using a Teflon glass homogenizer in 320 mM sucrose, 5 mM HEPES, pH 7.4 (adjusted with $\mathrm{NaOH}$ ). This was followed by a 2-step centrifugation, first in an SS34 rotor (Sorvall) for 12 minutes at $14,400 \times$ g, to pellet large tissue fragments, and then in a TLA100.3 rotor 
(Beckman) for 60 minutes at $264,000 \times \mathrm{g}$ to pellet all remaining cellular fragments. All centrifugation steps were performed at $4^{\circ} \mathrm{C}$. The fixatives were prepared so that the final amount of fixative in the solution with the cytosol was $4 \%$ PFA (pH 7, $\mathrm{pH} 4$ and 5) and $3 \%$ glyoxal. The samples were fixed for 15, 30, 45 or $60 \mathrm{~min}$ at room temperature (or $10 \mathrm{~min}$ at $37{ }^{\circ} \mathrm{C}$ for one of the PFA fixation controls). As control samples, cytosol was also fixed with PFA plus $0.2 \%$ glutaraldehyde and PFA plus $20 \%$ ethanol. After fixation samples were prepared for running on SDS polyacrylamide gels by adding $2 \mathrm{X}$ Laemmli sample buffer (Laemmli, 1970) and heating for $5 \mathrm{~min}$ to $95^{\circ} \mathrm{C} .10 \%$ polyacrylamide gels were prepared as described previously (Brunelle \& Green, 2014). $25 \mu$ of each sample and a non-fixed brain cytosol sample was run on the gels. The gels were stained in coomassie brilliant blue overnight and were destained for $2-3$ hours in $50 \%$ methanol, $40 \% \mathrm{H}_{2} \mathrm{O}, 10 \%$ acetic acid the following day. The stained gels were scanned and analyzed.

\section{Electron microscopy}

For electron microscopy of chemically fixed cells (Appendix Fig. S15), primary hippocampal neurons were fixed for $20 \mathrm{~min}$ on ice, and for another $20 \mathrm{~min}$ at room temperature, followed by quenching for $30 \mathrm{~min}$ in $100 \mathrm{mM} \mathrm{NH}_{4} \mathrm{Cl}$ and $100 \mathrm{mM}$ glycine. The neurons were then postfixed with $2.5 \%$ glutaraldehyde for $60 \mathrm{~min}$ at room temperature. Another $20 \mathrm{~min}$ of quenching in $\mathrm{NH}_{4} \mathrm{Cl}$ and glycine were followed by 60 min of incubation in $1 \%$ osmium tetroxide. Afterwards, the neurons were washed in filtered PBS for $15 \mathrm{~min}$, and were dehydrated with a series of ethanol dilutions. Subsequently, the cells were embedded in Epon resin by first incubating them for 3 hours in a 1:1 mixture of ethanol and resin, and then incubating in pure resin for 48 hours at $60^{\circ} \mathrm{C}$. The samples were cut into $80-100 \mathrm{~nm}$ sections using a LeicaEM UC6 ultramicrotome, and were mounted on copper 50-mesh grids (Plano GmbH \#2405C) or Formvar-coated copper slot grids (Plano GmbH \#G2500C). The thinsections were labeled with $1 \%$ uranyl acetate for $10 \mathrm{~min}$ and were afterwards washed for several minutes in $d_{d d H_{2}} \mathrm{O}$. The samples were imaged using a JEOL JEM1011 electron microscope (JEOL $\mathrm{GmbH}$ ), with a magnification of 10000 X.

For electron microscopy of high-pressure frozen samples (Appendix Fig. S15), primary hippocampal neurons were frozen using a Leica HPM100 high pressure freezer, using PBS with $20 \%$ Polyvinylpyrrolidone as filler solution. The samples were freeze-substituted as described before (McDonald \& Webb, 2011). Postfixation was done in a mixture of $1 \%$ glutaraldehyde, $1 \% \mathrm{OsO}_{4}$ and $1 \% \mathrm{H}_{2} \mathrm{O}$ (modified after Jiménez et al, 2006) prior to embedding in Epon via an Epon dilution series (McDonald \& Webb, 2011). The samples were cut into ultrathin sections (60 nm), stained in $1 \%$ uranyl acetate, and imaged with a Zeiss transmission electron microscope.

\section{Data analysis}

All data analyses were performed automatically or semi-automatically using Matlab (The Mathworks Inc.), with exception of the analysis from Appendix Fig. S12. Analyses in Fig. 1a, 1b, 3b, and in Appendix Fig. S1, S2, S3, S8, S9 and S16 were performed using custom-written Matlab routines that measure the average fluorescence intensity in manually selected 
regions. For Fig. 1a, 1b and Appendix Fig. S1 and S16, the regions were selected manually. For Fig. 3b, a Matlab routine was used to separate cells from each other, using the watershed transform, and to thus determine the cellular regions of interest.

The fluorescence signals of the GFP and of the immunostainings in Appendix Fig. S10 were measured by a Matlab automatic routine that first identified the GFP signals, by applying a threshold to remove background signals, and then measured the intensity of the immunostaininigs in the GFP-positive regions of interest. For all analyses of the signal intensity in terms of "signal over background", signal- and background-containing regions of interest were manually determined, before dividing the average intensity in the former by the average intensity in the latter.

The analysis of the DIC images in Fig. 2 was performed using a Matlab routine that calculated the correlation coefficients of circular regions of interest ( $\sim 500 \mathrm{~nm}$ in diameter), selected manually in the first image, to every other image taken throughout the 60 min of imaging. $\mathrm{A}$ similar analysis was performed for the fluorescent images from Appendix Fig. S4, using circular regions of interest centered on particular organelles, selected by the user. Again, the same analysis was performed for the GFP images, before and after fixation, from Appendix Fig. S6, and for the images of transferrin-labeled and immunostained cells (Appendix Fig. S11). The SDS-PAGE gels in Fig. 3a and Appendix Fig. S7 were analyzed by measuring the overall band intensity that is left after fixation compared to the non-fixed sample. The entire length of the lanes was measured, and the intensity was summed over all bands. To avoid the smear induced by fixed molecules, which is especially evident in glutaraldehyde fixation, the signal along the lanes was first subjected to a high-pass filter.

The efficiency of preserving mitochondria during fixation (Appendix Fig. S5) was analyzed by measuring the lengths of mitochondria before and after fixation. Regions of interest containing mitochondria were manually selected and the mitochondria were detected by a thresholding procedure. The mitochondria length was then determined automatically.

For the analysis of the electron microscopy images (Appendix Fig. S15), synaptic vesicles were selected manually, and line scans were applied to each vesicle.

For the analysis of the immunostained proteins in hippocampal neurons (Appendix Fig. S12 14), structures that appeared to be of organellar organization were identified and counted manually. This analysis was done blinded, randomizing both the order and the nature of the images. The number of objects was counted per immunostained $\mu \mathrm{m}^{2}$, in order to take into account, the different amounts of neuronal structures per image.

To analyze the structure of the observed objects, 100 typical objects were selected by an experienced observer, again in a blind fashion. The objects were clicked on, to select the center of the area. Square regions of interest, of several $\mu \mathrm{m}$ in width, were automatically generated, centered on the selected objects, and were preserved for further analysis. After all objects were selected, the regions of interest were overlaid, and each was rotated in turn (in $5^{\circ}$ increments, using both the real image and a mirrored image), until the best possible alignment to the other regions of interest was obtained. Only the area within $1 \mu \mathrm{m}$ from the region of interest center was used in measuring the alignments, to restrict the alignment 
analysis to the selected object, and not to other objects that may have been present in the regions of interest. The strength of the alignment was verified by calculating the Pearson's correlation coefficient at every angle. Once a best fit was found (with the maximal Pearson's coefficient), all images were summed, and the average object was thus obtained (shown in Appendix Figs. S13 and S14). Line scans, obtained by drawing horizontal lines through the individual typical objects (after rotation) are shown in the graphs in these figures (in the form of mean \pm SEM of all 100 line scans through the 100 typical objects).

\section{Statistics}

Typically, measurements were performed over multiple cells and experiments. For experiments studying multiple cells, such as neuronal immunostainings, we typically used at least 10 individual neurons in each analysis. For experiments involving single cells (such as time series obtained on one cell), we performed at least 3 independent experiments. For biochemical experiments, multiple experiments were performed (2-7). The sample numbers were increased if substantial variation was noted in the initial experiments. All graphs depicted here were generated using Sigma Plot (Systat Software, Inc). All bar graphs show mean values and all error bars represent the standard error of the mean (SEM), calculated in Sigma Plot (except for the quantification of cardiomyocyte stainings in Fig.9, which represents mean values with standard deviation values). For statistical analyses in Fig. $3 a$ and Appendix Fig. S16 (multiple comparisons), a one-way ANOVA with a post-hoc Tukey test was performed. For all other statistical analyses, the two-sided student's t-test (unpaired) or Wilcoxon rank sum test was applied to the data using the in-built function in Excel or using Matlab. For Figure 1 and Appendix Figs. S4 and S5 the number of independent experiments tested $(\mathrm{N})$ was below 5 . The t-test was chosen, assuming that the results come from a normal distribution. The justification for this assumption is that the variation between experiments is solely driven by experimenter (pipetting) errors, which are considered to be normally distributed. For larger data sets we used the Jarque-Bera test to verify the normal distribution of the data points. If the Jarque-Bera tests indicated normal distributions, we used t-tests for verifying differences between the samples. If one or both of the distributions were different from the normal distribution, according to the Jarque-Bera tests, we used a two-sample Wilcoxon rank sum test to verify differences between the samples.

For display purposes images were adjusted in brightness and contrast using ImageJ (Wayne Rasband, US National Institutes of Health). If intensities were compared, image adjustments in brightness and contrast were equally applied to all conditions.

\section{Animals}

P14 to P18 and adult wild type mice (Mus musculus) from the substrain C57BI/6J were obtained from the University Medical Center Göttingen. Newborn wild type Wistar rats (Rattus norvegicus) for the preparation of primary hippocampal neuron cultures were obtained from the University Medical Center Göttingen as well. Drosophila melanogaster of the Canton S strain were maintained in the laboratory, using conventional methods. 
All animals were handled according to the specifications of the University of Göttingen and of the local authority, the State of Lower Saxony (Landesamt für Verbraucherschutz, LAVES, Braunschweig, Germany).

Methods of collaborating labs can be found in the Appendix.

\subsection{Appendix}

\section{Appendix for "Glyoxal as an alternative fixative to formaldehyde in immunostaining and super-resolution microscopy"}

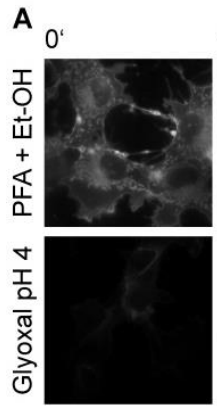

10
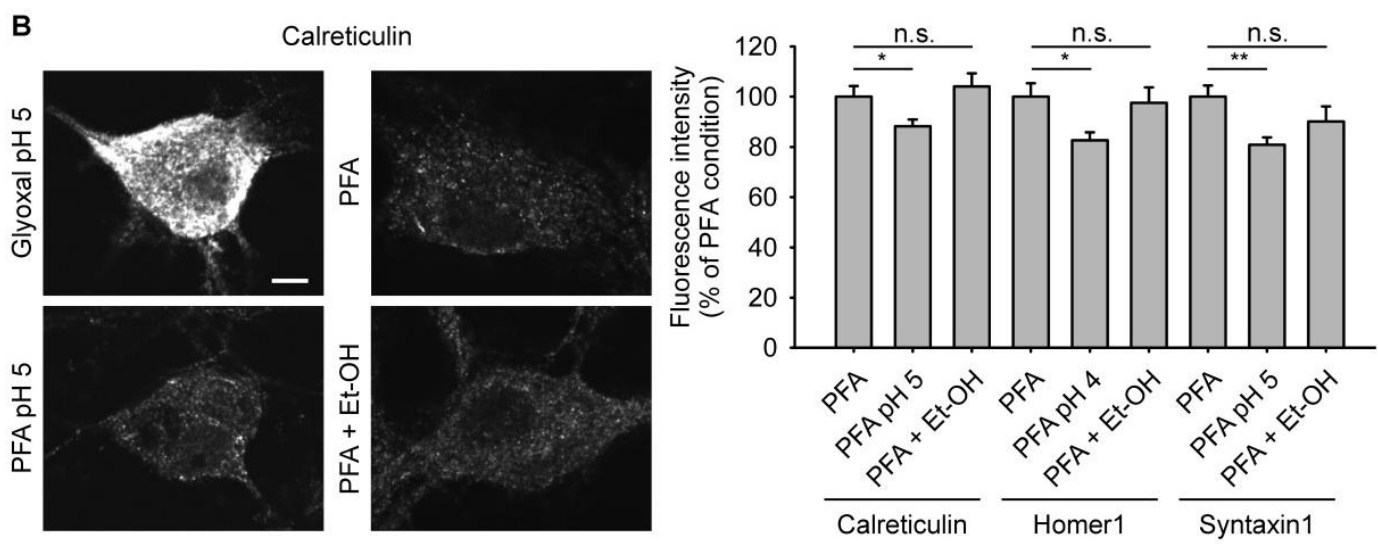

Appendix Figure S1 Addition of ethanol increases the speed of membrane penetration, but neither ethanol nor low $\mathrm{pH}$ improves immunostainings.

A The images show the entry of FM 1-43 into fibroblasts during the first 10 min of fixation. In addition to fixation with PFA and glyoxal pH 5 (as shown in Fig. 1b), we tested fixation with glyoxal pH 4 , and with PFA + ethanol. In the graphs either individual data points or mean values with SEM are plotted. $\mathrm{N}=2$ independent experiments for glyoxal $\mathrm{pH} 4$ and for PFA + ethanol. Scale bar $=20 \mu \mathrm{m}$.

$\mathrm{B}$ For testing the influence of EtOH and low pH on fixation with PFA and immunostaining, primary hippocampal neurons were fixed either with conventional PFA buffers (no ethanol, pH 7), with PFA containing the same amount of EtOH as our glyoxal-based fixatives, or with PFA at low pH. Immunostainings for calreticulin, homer 1 and syntaxin 1 show that neither the addition of EtOH, nor the low $\mathrm{pH}$ increase the quality of immunostainings. The quantification of fluorescence intensity even shows a reduced signal for samples fixed with PFA at low $\mathrm{pH} . \mathrm{N}=9-15$ cell regions per condition (calreticulin, syntaxin 1), focusing on large areas that contained the widely diffused signal for these two proteins. For homer 1 we analyzed smaller regions containing synapses; $68-80$ cell regions per condition. Scale bar $=5 \mu \mathrm{m} .{ }^{*} \mathrm{p}<0.05, * * p<0.01$. 

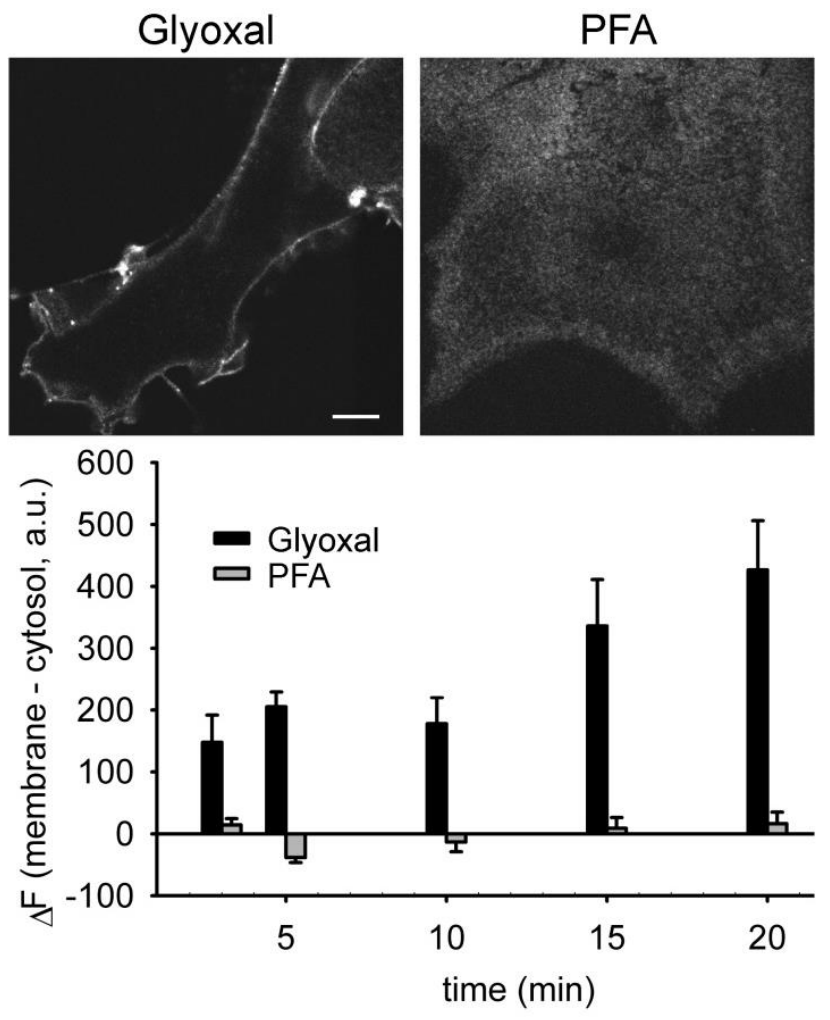

Appendix Figure S2 Fixation of Alexa Fluor 594-coupled transferrin, applied during fixation.

COS-7 cells were fixed at $37{ }^{\circ} \mathrm{C}$ for $3,5,10,15$ and $20 \mathrm{~min}$, before transferrin-Alexa Fluor 594 was added, and the cells were incubated in the fixative and transferrin for another $20 \mathrm{~min}$. The fluorescence intensity was measured in the cytosol of the cells and on the plasma membrane. The graph shows the fluorescence intensity at the membrane, from which the fluorescence intensity in the cytosol was subtracted. This analysis reveals that transferrin is fixed on the plasma membrane by glyoxal, whereas PFA-fixed cells show transferrin both on the plasma membrane and in the cytosol. $\mathrm{N}$ $=8-24$ cellular ROIs per condition. Scale bar $=5 \mu \mathrm{m}$. 


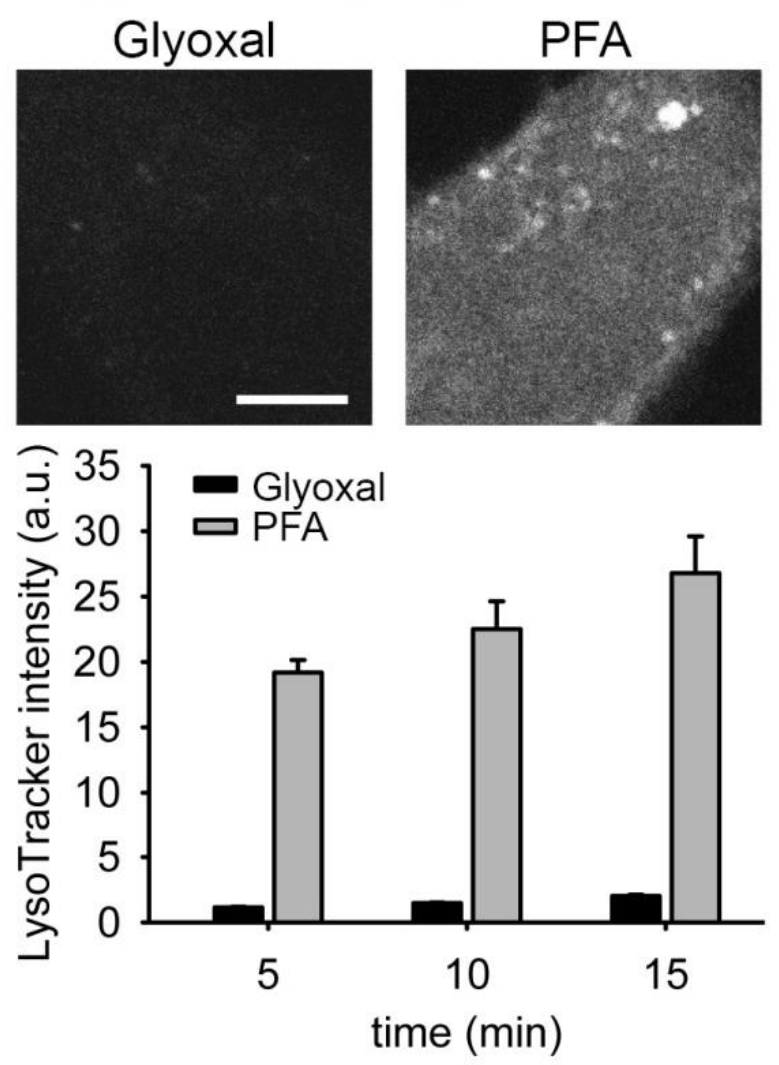

Appendix Figure S3 Maintenance of the acidic lumen of lysosomes after fixation with PFA or glyoxal for different durations.

HeLa cells were fixed at $37^{\circ} \mathrm{C}$ for 5,10 and 15 min before LysoTracker was added, and the cells were incubated in the fixative and LysoTracker for another $20 \mathrm{~min}$. The measured LysoTracker intensity reveals the maintenance of the acidic lumen of lysosomes in PFA-fixed cells, whereas in glyoxal-fixed cells the acidic compartments are not detected. $N=9-21$ cells per condition. Scale bar $=5 \mu \mathrm{m}$. 
Transferrin uptake Glyoxal
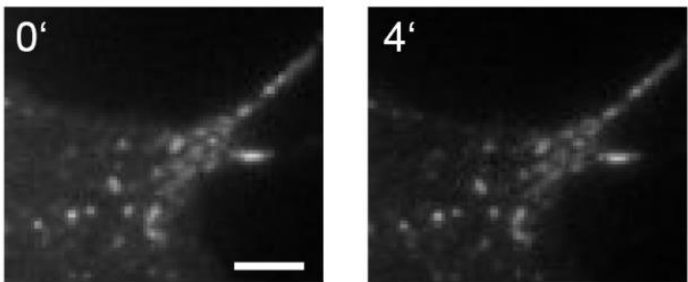

Transferrin uptake

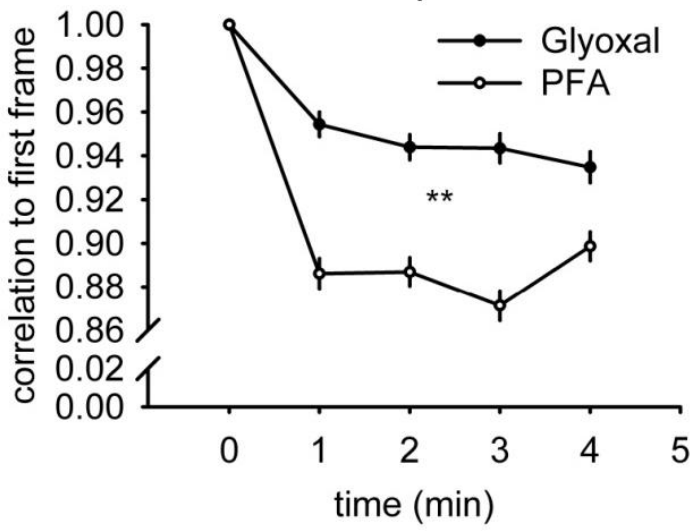

Transferrin uptake PFA
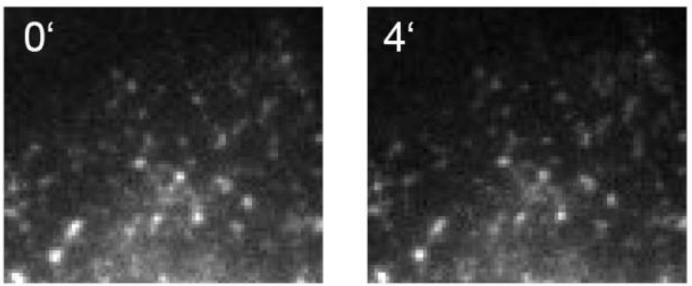

Cholera toxin uptake

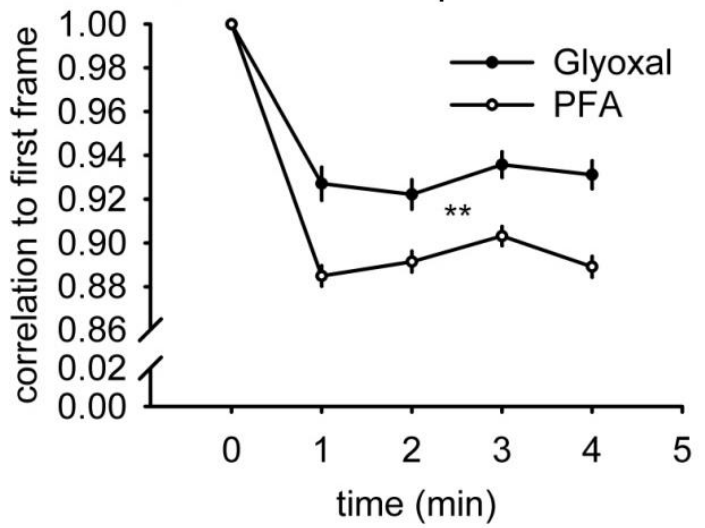

Appendix Figure S4 Transferrin and cholera toxin uptake during fixation.

COS-7 and HeLa cells were incubated with transferrin-Alexa Fluor594 or cholera toxin subunit B-Alexa Fluor555 for $10 \mathrm{~min}$ at $37{ }^{\circ} \mathrm{C}$, and were imaged during the first few min after the addition of the fixative, during which time it is likely that active cellular movements still take place. Representative images are shown from the transferrin uptake experiment. The graphs show the correlation to the first frame of each image taken throughout the first minutes of fixation. The high correlation coefficients show that both fixatives allow little endosome movement, though glyoxal seems to act slightly faster than PFA. $\mathrm{N}=49-325$ cellular regions from $3-4$ independent experiments. Scale bar $=5$ $\mu \mathrm{m} .{ }^{* *} \mathrm{p}<0.01$ 

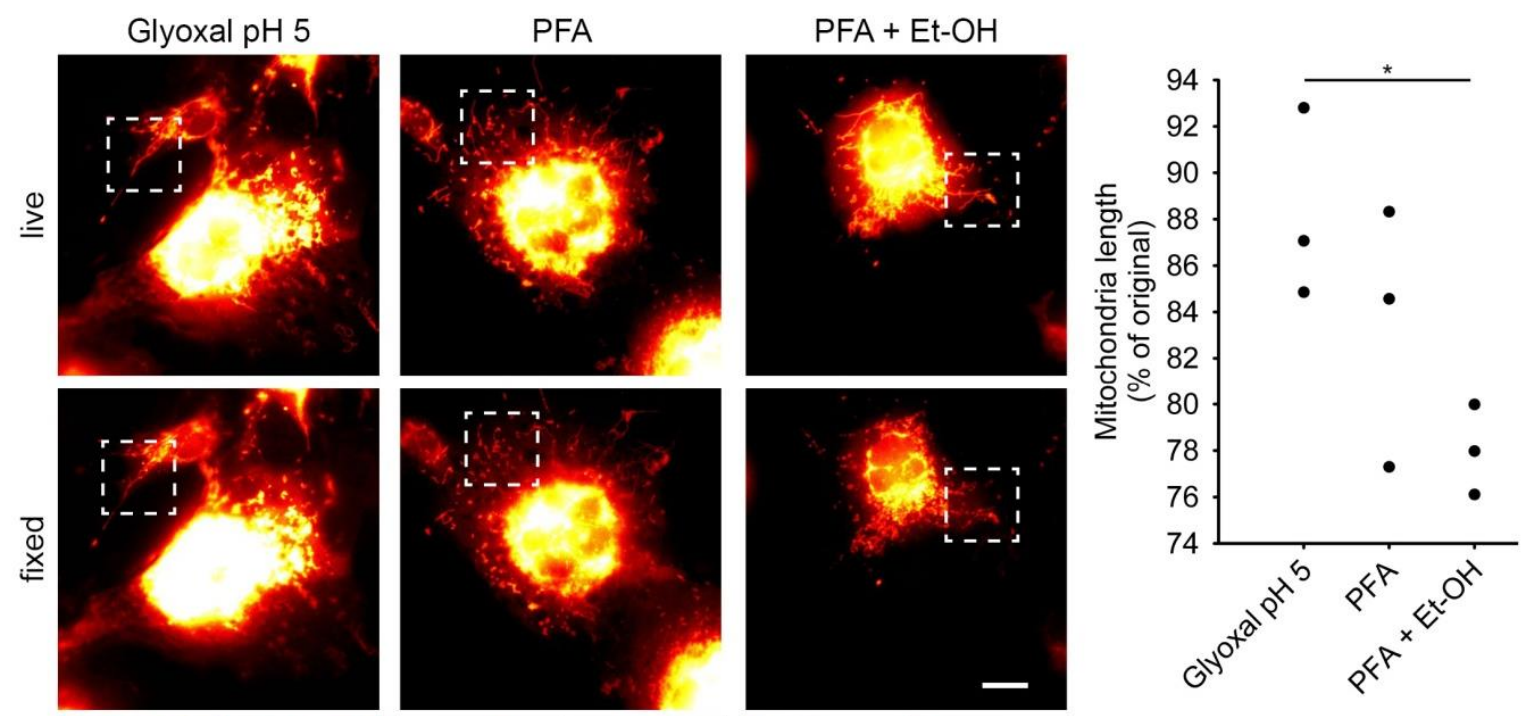

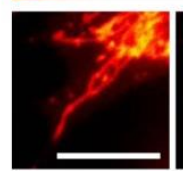

live

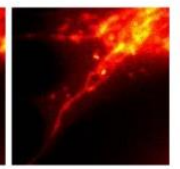

fixed

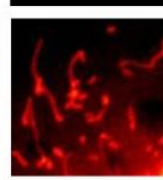

live

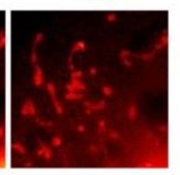

fixed

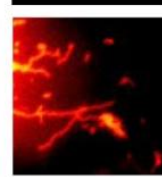

live

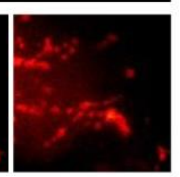

fixed

\section{Appendix Figure S5 Preservation of mitochondria after $\mathbf{6 0}$ min of fixation.}

Fibroblasts were transfected with the mitochondrial marker TOMM70, linked to GFP, and were imaged before and after fixation. The graph indicates the decrease in mitochondria size, caused by fragmentation during fixation (visible in the zoom-ins at the bottom of the panels). The mitochondria preservation was not significantly different between PFA and glyoxal, but was significantly different between glyoxal and PFA with added ethanol. This suggests that the ethanol addition to PFA does not increase its fixation accuracy. $\mathrm{N}=3$ experiments. Scale bar $=20 \mu \mathrm{m} .{ }^{*} \mathrm{p}<0.05$. 

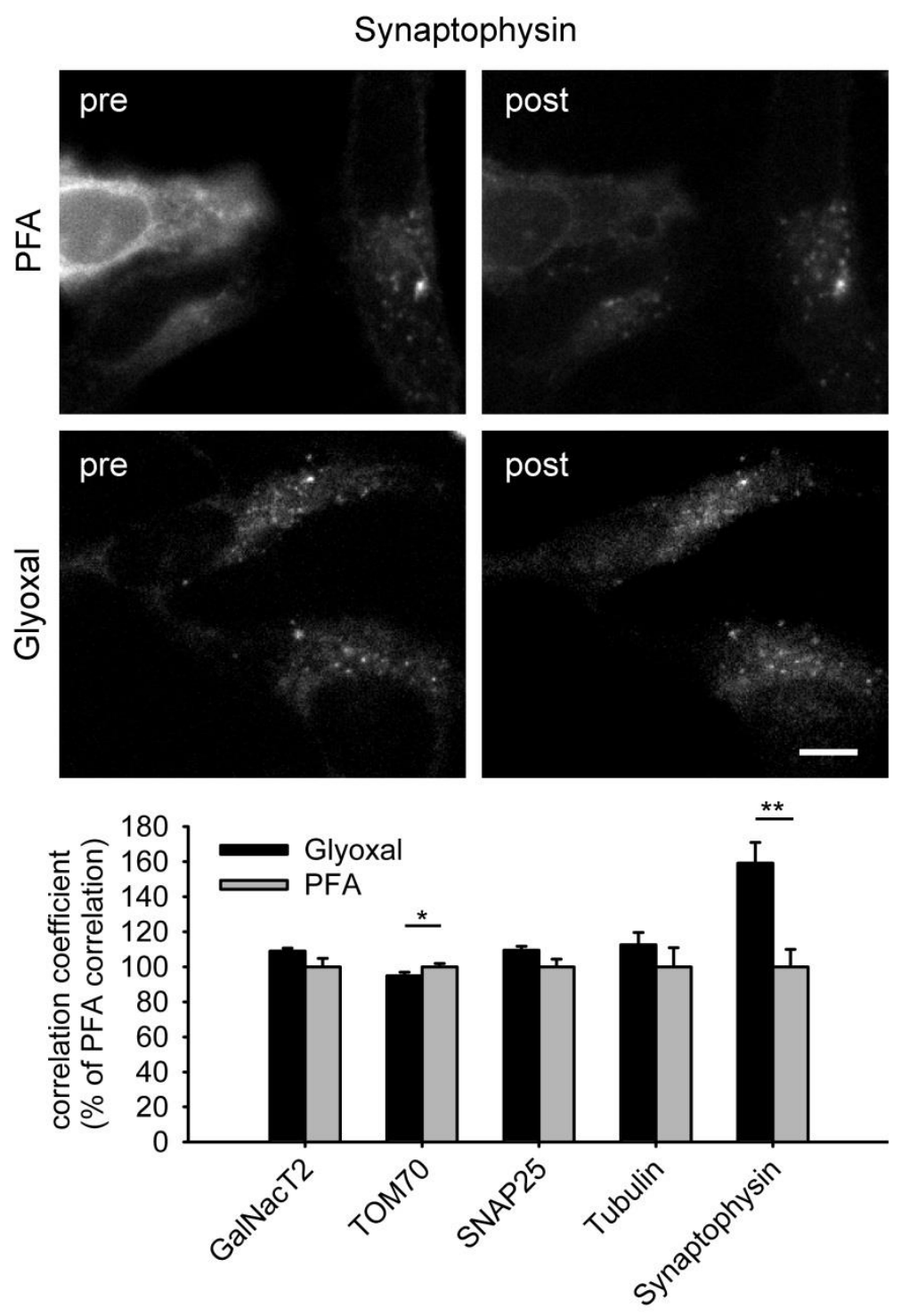

Appendix Figure S6 Preservation of cellular structures by PFA and glyoxal fixation.

Various GFP-tagged proteins were expressed in COS-7 and HeLa cells. The preservation of structures/organelles was assessed by measuring the correlation between the GFP signals prior to, and after 60 min of fixation with either PFA or glyoxal. The graph shows the correlation of the GFP signals pre and post fixation. $N=13-51$ cellular ROls per condition. Scale bar $=10 \mu \mathrm{m} .{ }^{*} p<0.05, * * p$ $<0.01$. 


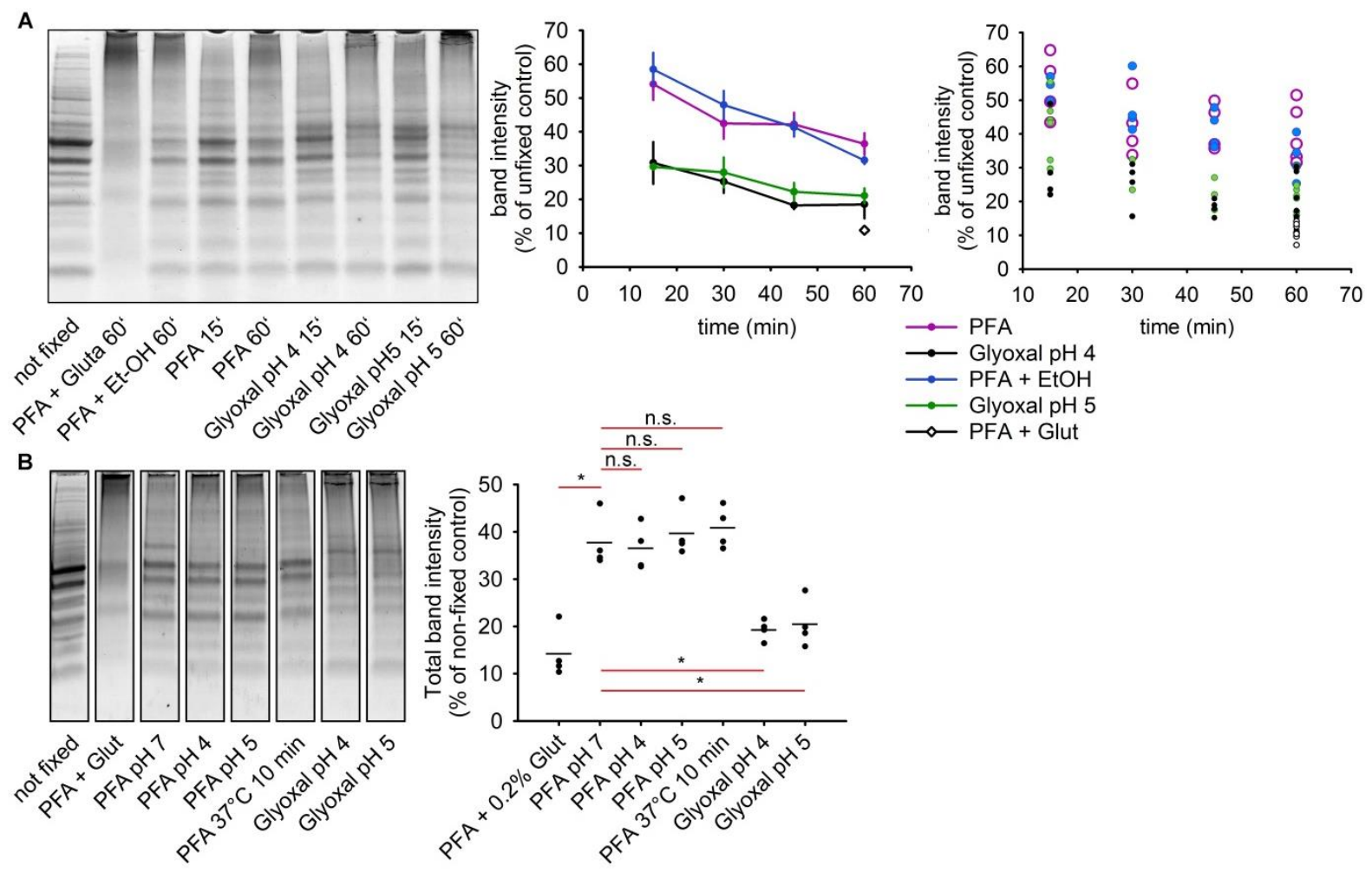

Appendix Figure S7 Comparison of glyoxal fixation with various alternatives of PFA fixation.

A Full polyacrylamide gel from figure $3 \mathrm{~A}$. In addition to fixation for 60 minutes with PFA, PFA and glutaraldehyde, glyoxal pH 4, and glyoxal $\mathrm{pH} 5$, which were shown in figure 3 , samples were also fixed for 15, 30 and 45 minutes (gels with fixation duration of 30 and $45 \mathrm{~min}$ are not shown here). We also fixed samples with PFA + ethanol. The graph shows the remaining intensities of all bands, for all tested time points (either individual data points or mean values with SEM are plotted). This indicates that fixation for shorter durations results in lower fixation strength. Additionally, fixation with PFA + ethanol does not increase the efficiency of fixation, in comparison with PFA alone. $N=$ between 2 and 7 experiments per condition.

$B$ Testing the efficiency of PFA fixation under different alternative conditions $\left(\mathrm{pH} 4, \mathrm{pH} 5\right.$ or at $\left.37^{\circ} \mathrm{C}\right)$ in the SDS PAGE based approach (see also figure 3). A quantification of total band intensity left after fixation shows that neither low $\mathrm{pH}$, nor higher temperature increase fixation efficiency significantly, when compared to PFA pH 7 fixation. Glyoxal fixation, which was tested in parallel to these experiments in the same gels, reduced the total band intensity significantly, when compared to these PFA conditions, as in all other SDS PAGE experiments we tested. $\mathrm{N}=4$ gels in 4 independent experiments. $* p<0.05$ 


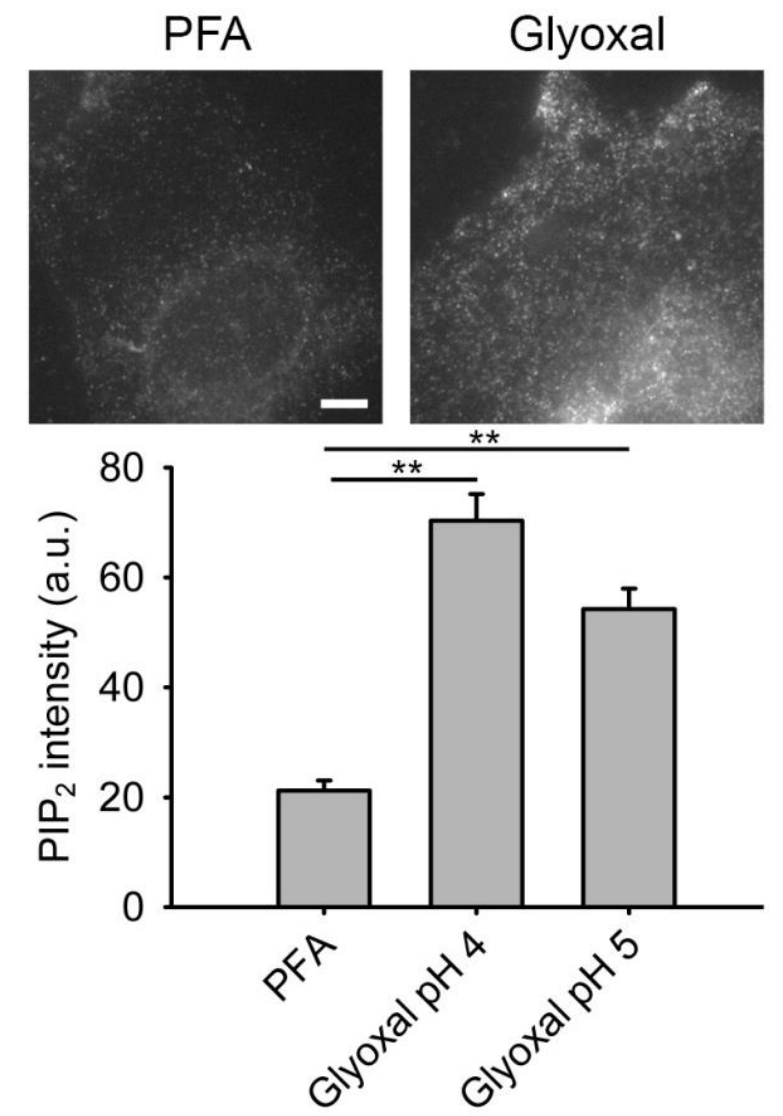

Appendix Figure S8 Fixation of lipids by PFA and glyoxal.

PFA and glyoxal-fixed COS-7 cells were immunostained for Phosphatidylinositol-4,5-bisphosphat $\left(\mathrm{PIP}_{2}\right)$, and the fluorescence intensity was measured. Cells that were fixed with glyoxal exhibited significantly brighter stainings than PFA-fixed cells. $N=18-35$ cells. Scale bar $=5 \mu \mathrm{m}$. ${ }^{* *} p<0.01$. 


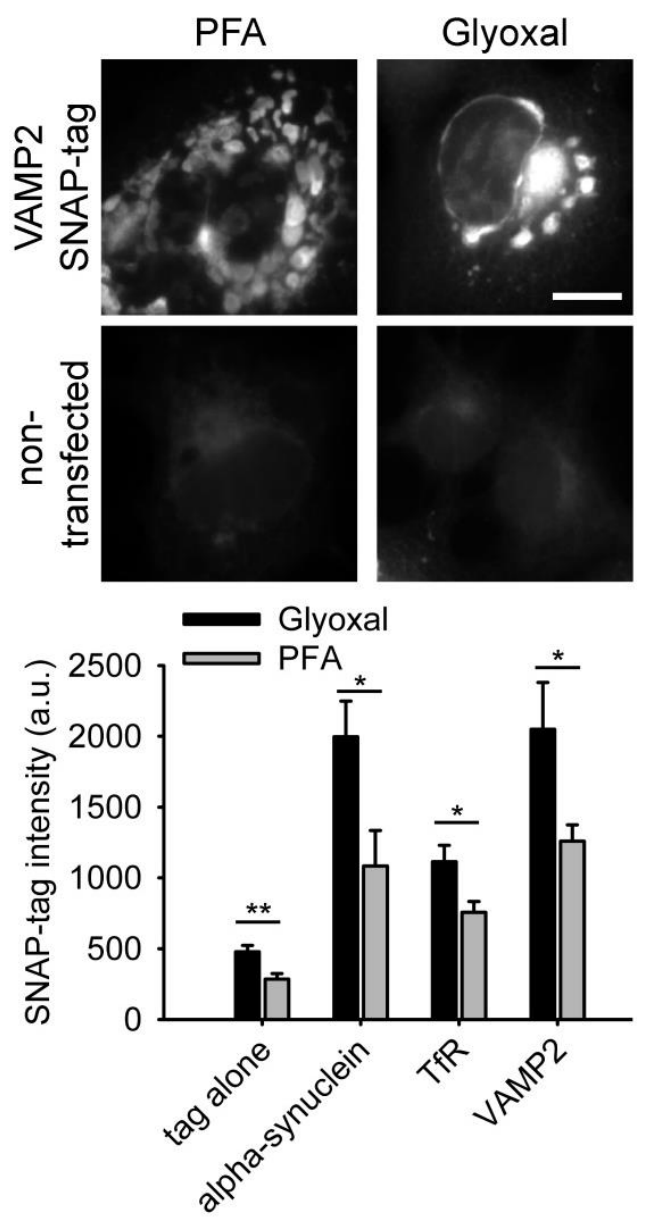

Appendix Figure S9 Preservation of enzymatic activity after PFA and glyoxal fixation.

HeLa cells were transfected with the enzymatic labeling tag SNAP-tag, either alone or linked to three different cellular proteins. After fixation the maintenance of the enzymatic activity was tested by applying a fluorophore that is covalently linked to the tag by its own enzymatic reaction. All tested SNAP-tag variants show significantly higher labeling for glyoxal-fixed cells compared to PFA-fixed cells. Non-transfected cells show no substantial fluorescence. $N=10-14$ images analyzed per condition (each containing multiple cells). Scale bar $=2 \mu \mathrm{m} .{ }^{*} p<0.05, * * p<0.01$. 

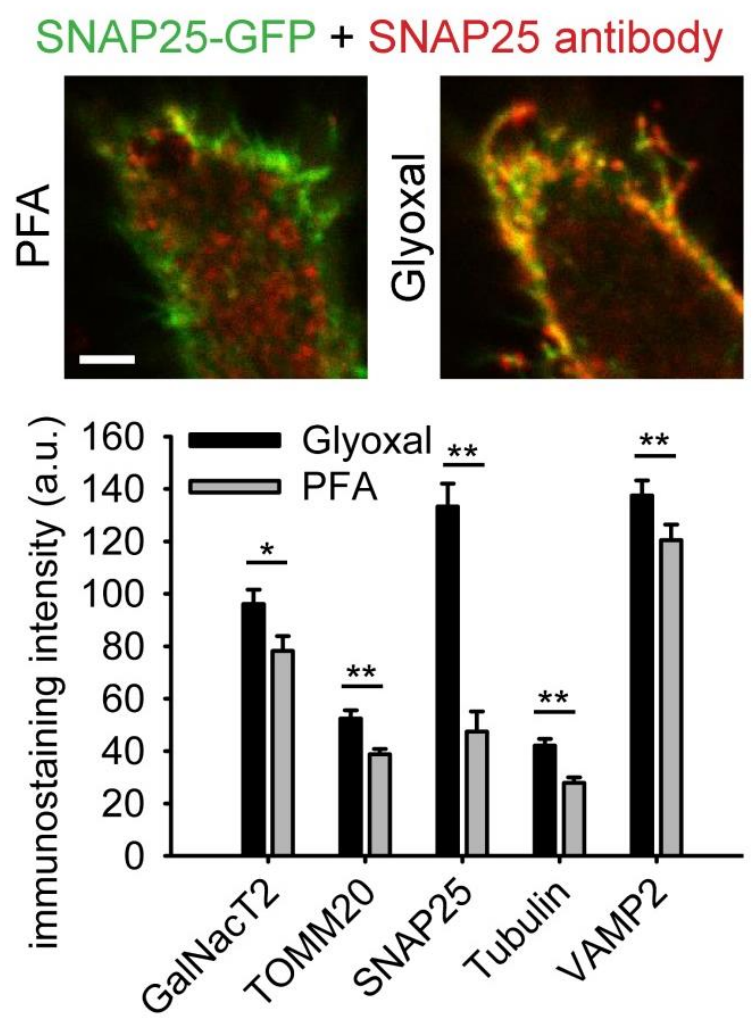

Appendix Figure S10 Immunostaining efficiency after PFA and glyoxal fixation.

COS-7, HeLa or BHK cells overexpressing different GFP-tagged proteins were fixed and immunostained, and the fluorescence intensity of the antibody staining was measured. Immunostaining intensities for all targets were significantly higher in glyoxal-fixed cells than in PFAfixed cells. $N=5-330$ cellular ROIs per condition. Scale bar $=2 \mu \mathrm{m} .{ }^{*} p<0.05,{ }^{* *} p<0.01$. 

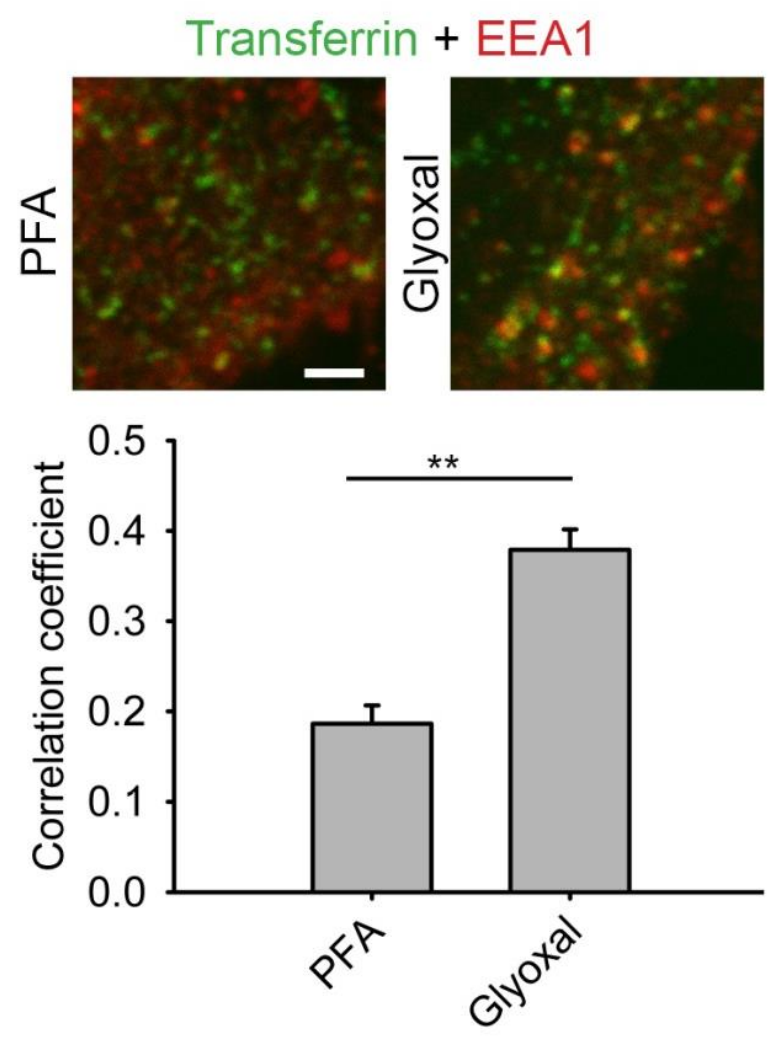

Appendix Figure S11 Preservation and antibody recognition of cellular targets fixed by PFA and glyoxal.

HeLa cells that took up fluorescently-labeled transferrin were immunoassayed for the endosomal marker EEA1. The correlation of transferrin, which should be taken up into endosomes, to the immunoassayed EEA1 is significantly higher for glyoxal-fixed cells than for PFA-fixed cells. $N=15$ (PFA) and 29 (glyoxal) ROIs analyzed. Scale bar $=2 \mu \mathrm{m} .{ }^{* *} \mathrm{p}<0.01$. 


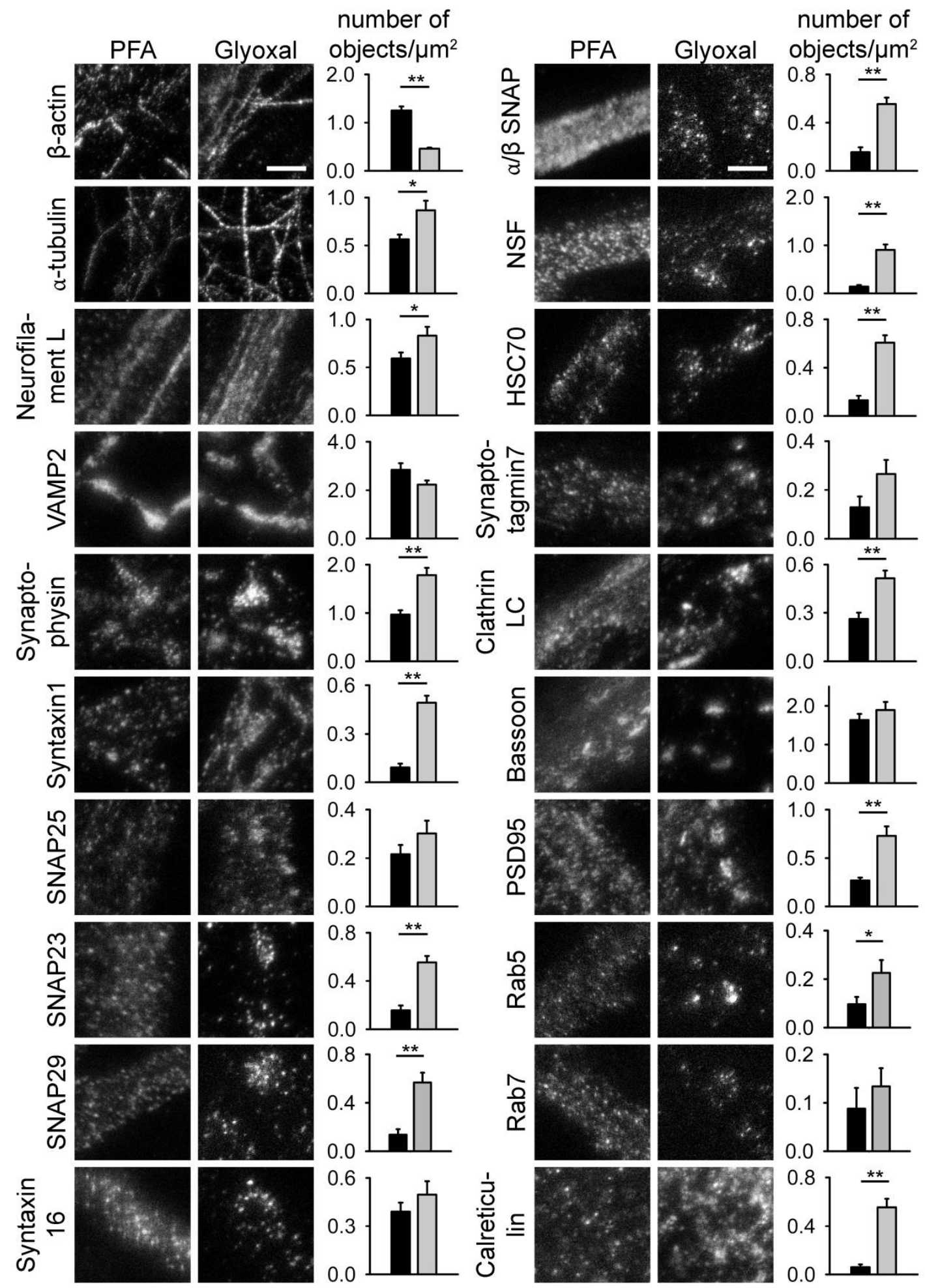

Appendix Figure S12 Super-resolution imaging of 20 immunostained proteins in cultured neurons after fixation with PFA or glyoxal.

Typical images of stained hippocampal neurons are shown, after glyoxal or PFA fixation. The bar graphs indicate the number of organelle-like structures that an experienced user could detect, per stained surface area (black bars $=$ PFA, grey bars $=$ glyoxal). Significantly more objects were identified in glyoxal than in PFA fixed samples for the following proteins: $\alpha$-tubulin, neurofilament $L$, synaptophysin, syntaxin 1 , SNAP23, SNAP29, $\alpha / \beta$ SNAP, NSF, HSC70, clathrin light chain, PSD95, rab5 and calreticulin; $N=7-15$. For $\beta$-actin, significantly more structures were found in PFA-fixed cells, since the actin filaments appear more fragmented after this treatment, which increases the number 
of observed strands (see analysis in Appendix Figure S13). Scale bar $=2.5 \mu \mathrm{m}$ (for $\beta$-actin and $\alpha$ tubulin) and $1 \mu \mathrm{m}$ (for the rest). ${ }^{*} \mathrm{p}<0.05,{ }^{* *} \mathrm{p}<0.01$. All experiments were analyzed in a blind fashion (see Methods for details).

In detail, we analyzed several types of proteins. We started with immunostainings for $\beta$-actin, $\alpha$ tubulin, and neurofilament L, which are expected to form linear (filamentous) structures. Significantly more microtubules and neurofilaments could be detected after glyoxal fixation. In contrast, the number of actin filaments was significantly higher after PFA fixation, due to their fragmentation (analyzed in Appendix Fig. S13). We then analyzed two markers of synaptic vesicles, VAMP2 and synaptophysin. The vesicles form clusters in synaptic boutons. The same number of boutons could be observed in VAMP2 immunostainings, for both glyoxal and PFA fixation. For synaptophysin, however, significantly more boutons could be identified after glyoxal fixation. We next focused on five SNARE proteins, which are present in the synaptic plasma membrane (syntaxin 1, SNAP25), in other sites of the neuronal plasma membrane (SNAP23, SNAP29), or in endosomes (syntaxin 16). SNARE proteins form domains in the membranes (see for example (Bethani et al, 2007; Lang \& Rizzoli, 2010). We observed far more domains of syntaxin 1 after glyoxal fixation. Similar, albeit less pronounced, effects were observed for SNAP23 and SNAP29. No significant differences were observed for syntaxin 16 and SNAP25. Several soluble and/or organelle-attached proteins also provided more detailed (less uniform) images after glyoxal fixation, in which significantly more organelle-like structures could be detected ( $\alpha / \beta$-SNAP, NSF, Hsc70, clathrin, Rab5, and Rab7). The number of observed organelle-like objects were lower in PFA samples for several targets that are anchored to the membrane or to other cellular structures, including syntaxin 1, as mentioned above, and also the ER marker calreticulin and the active zone protein PSD95. Some such targets were detected equally well after both PFA or glyoxal fixation, including synaptotagmin 7 , which is associated to vesicles, or the active zone component Bassoon. 

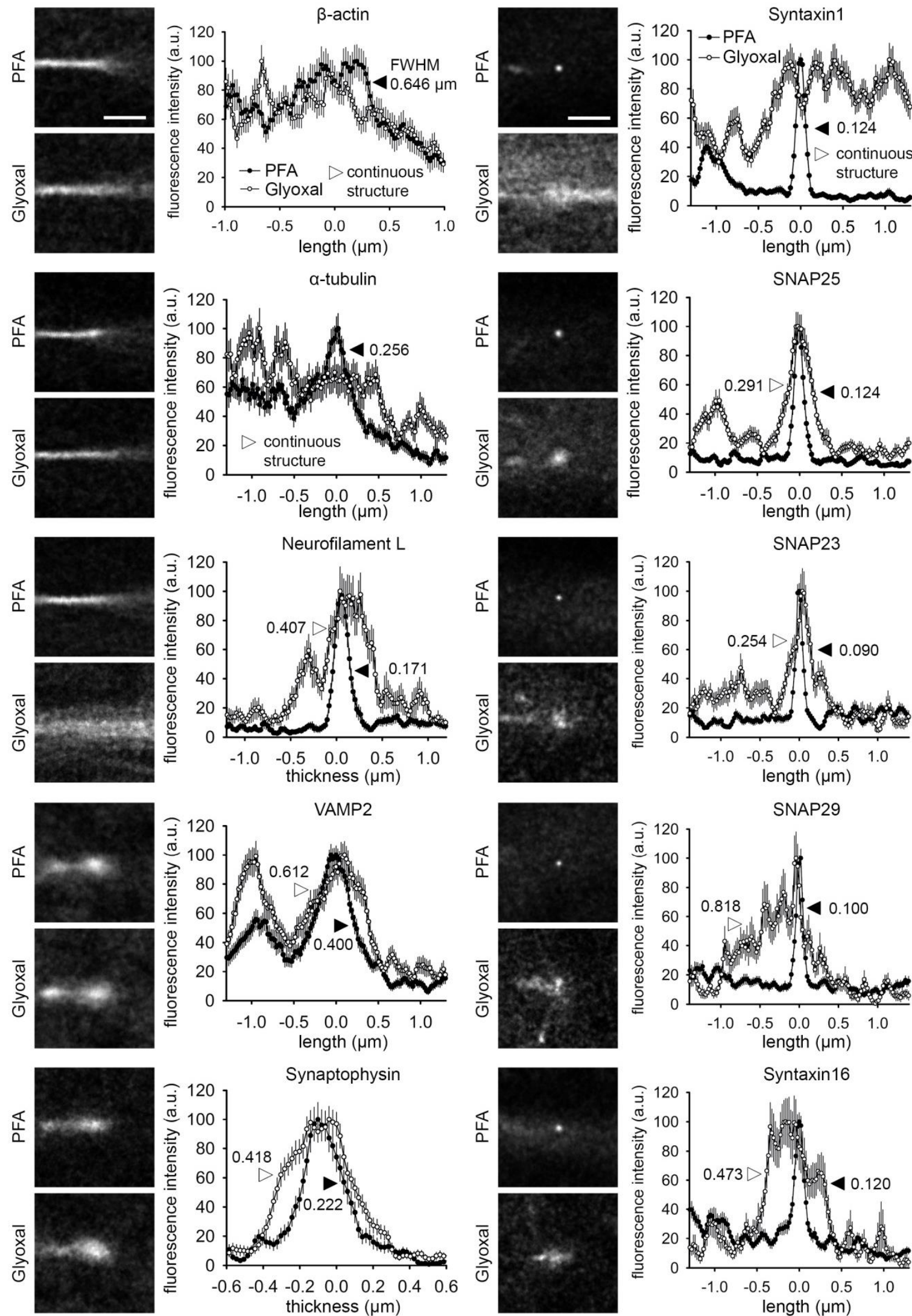

Appendix Figure S13 Ten pairs of average objects (structures) from neurons immunostained after PFA or glyoxal fixation.

To further analyze the difference between the glyoxal- and PFA-fixed samples, we created average objects, or average target structures, by overlaying 100 typical objects for every staining from Appendix Fig. S12 (again in a blind fashion). The objects were all centered, and were rotated to 
create a maximal alignment. This procedure is used in super-resolution microscopy to reveal aspects of the average object that may be missed by the visual investigation of only single objects (see for example (Löschberger et al, 2012; Revelo et al, 2014; Wilhelm et al, 2014). The typical objects were aligned and averaged, and the resulting averages are shown in the images in Appendix Fig. S13 and S14, in the same order as in the two columns of Appendix Fig. S12. 10 target proteins are included in each of these two figures. Vertical or horizontal line scans indicate the length of the objects, with the exception of neurofilament $L$, and synaptophysin, for which the thickness of the objects is indicated. The arrowheads (black $=$ PFA, white $=$ glyoxal) and the numbers indicate the size of the objects, in the form of the full width at half maximum (FWHM) of the linescans (in $\mu \mathrm{m}$ ). For some graphs the FWHM could not be measured, since there was no prominent single peak present, meaning that the objects consisted of continuous structures. This is noted on the respective graphs, as in the case of syntaxin 1 , which is an abundant and widely distributed protein, which is expected to be present in large neuronal areas. The error bars show the SEMs of the line scans $(n=100$ objects). Scale bar $=1 \mu \mathrm{m}$.

A simple overall conclusion is that the average object after PFA fixation is represented by a spot of $\sim 70$ to $120 \mathrm{~nm}$ in diameter for 14 of the proteins: syntaxin 1, SNAP25, SNAP23, SNAP29, syntaxin 16, $\alpha / \beta-S N A P, N S F$, HSC70, synaptotagmin 7, clathrin, PSD95, Rab5, Rab7, and calreticulin. Such small objects overlap in size with spots created by clusters composed of one primary antibody and the secondary antibodies that bind it (in our hands, at the resolution of the STED microscope used here (Opazo et al, 2010); 2-3 secondary antibodies bind each primary in our experiments (Opazo et al, 2010). For all of these proteins larger average objects were found after glyoxal fixation, which are too large to be merely single antibody spots. For the remaining 6 target proteins ( $\beta$-actin, $\alpha$-tubulin, neurofilament L, VAMP2, synaptophysin, and Bassoon), PFA revealed objects that were similar to those observed in the glyoxal-fixed samples. Actin filaments and microtubuli, however, were substantially shorter after PFA fixation, which implies that they became fragmented during fixation. Neurofilaments were far thinner after PFA fixation than after glyoxal fixation. Synaptic boutons were revealed by VAMP2 or synaptophysin antibodies after both PFA and glyoxal fixation, as mentioned in the previous section, but they were shorter and/or thinner after PFA fixation, suggesting that fewer epitopes were revealed. Finally, active zones were recognizable in the staining for the marker Bassoon, for both PFA and glyoxal, but they appeared larger after glyoxal fixation (Appendix Fig. S14). 

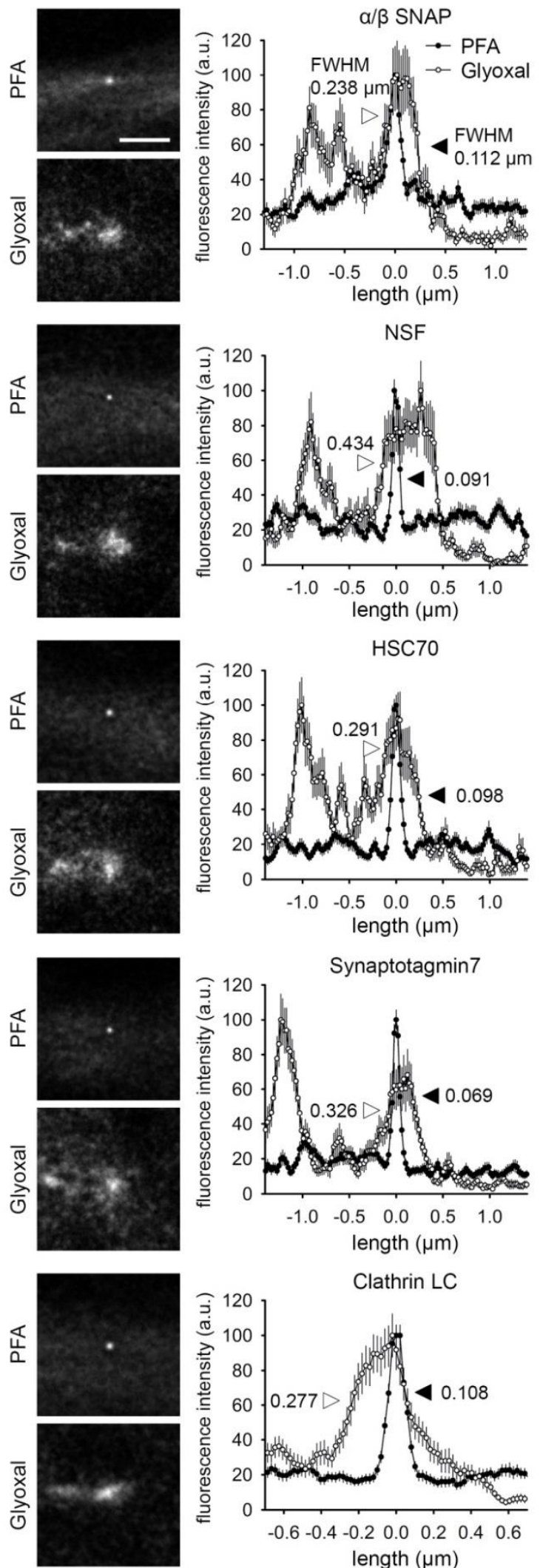
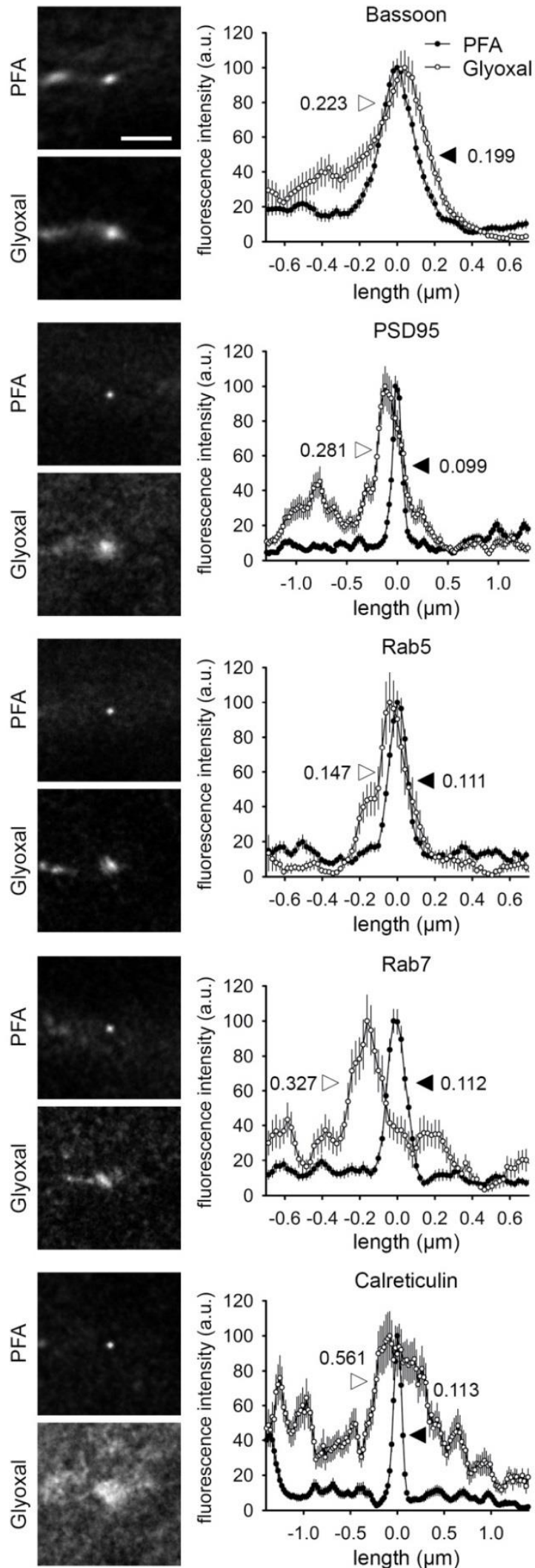

Appendix Figure S14 Ten additional pairs of averaged objects from neurons immunostained after PFA or glyoxal fixation (continuation of Appendix Figure S13).

The analysis was performed exactly as in Appendix Figure S13. Arrowheads (black = PFA, white = glyoxal) and numbers indicate the FWHM of the linescans (in $\mu \mathrm{m}$ ), as in Appendix Figure S13. Scale bar $=1 \mu \mathrm{m}$. 
PFA
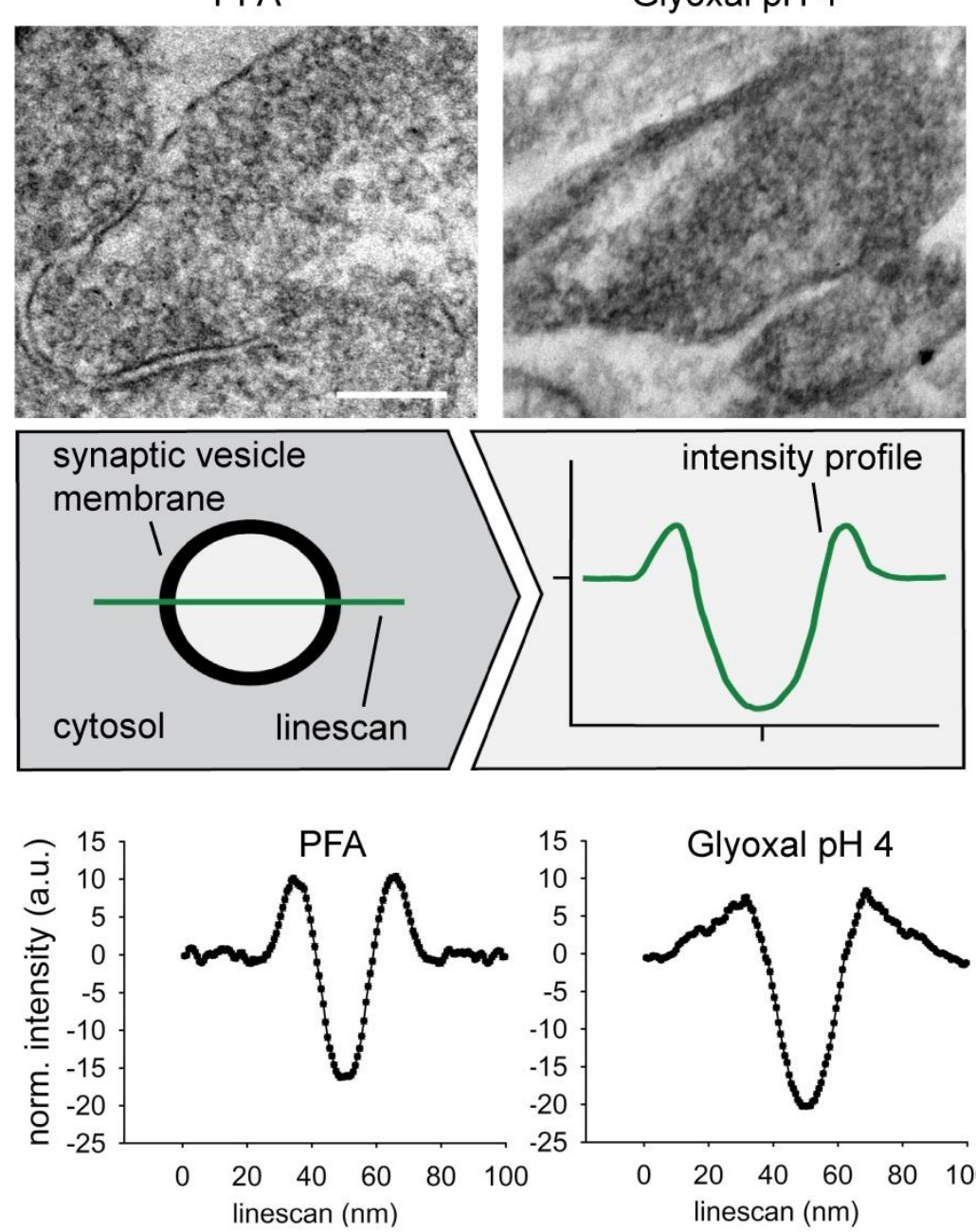

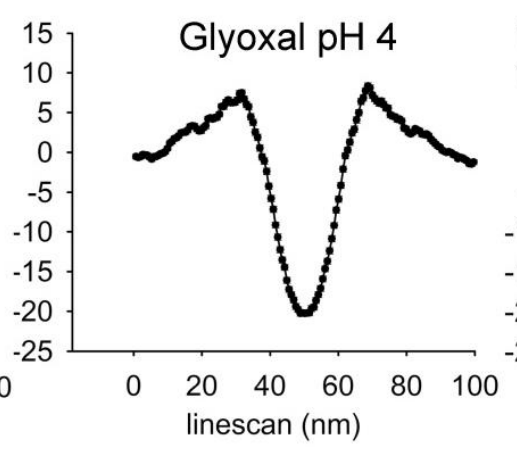

High pressure freezing
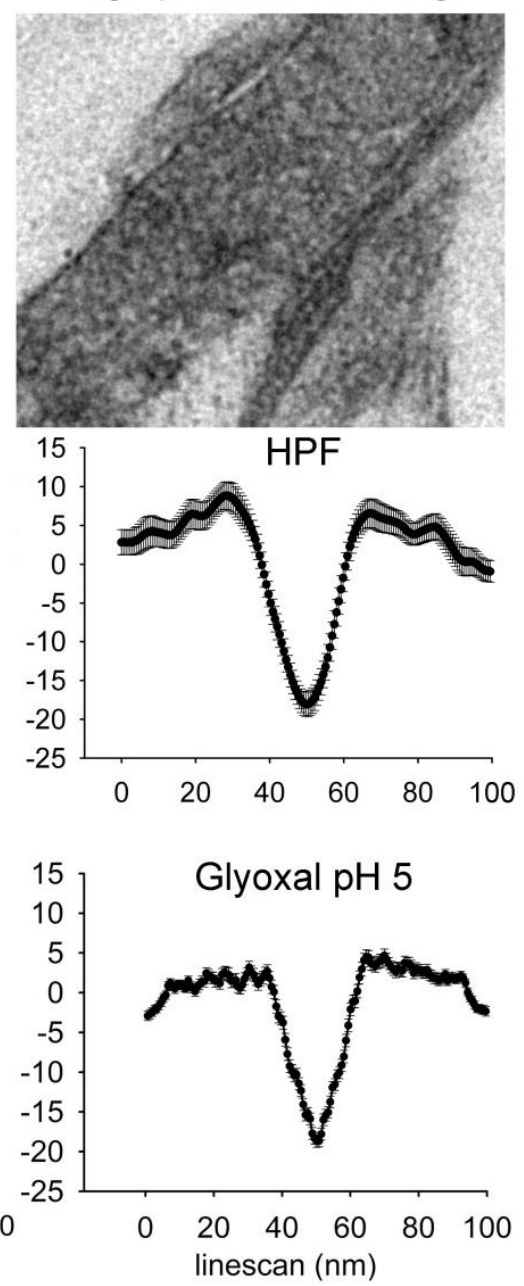

Appendix Figure S15 Electron microscopy of PFA- or glyoxal-fixed hippocampal neurons.

Representative images of synaptic boutons fixed with PFA or glyoxal, in cultured hippocampal neurons, are shown. For comparison, a representative image of cultured hippocampal neurons fixed via high-pressure freezing (HPF) and freeze-substitution is shown in the right panel. The graphs indicate average line scans performed through synaptic vesicles of the fixed neurons (see cartoon), \pm SEM (note that the SEMs are almost as small as the graph dots for the chemical fixed samples). The intensity was normalized to the background intensity of the images, outside of the vesicles. The line scan has a minimum in the center, corresponding to the center of the synaptic vesicle lumen, which is devoid of proteins. The vesicle membrane is clearly visible, and is significantly more electron-dense than the baseline outside the vesicles in the PFA-fixed samples, suggesting that the cytosol contains fewer proteins than the membrane of the vesicles in this condition. This effect is far less pronounced in glyoxal-fixed samples and the HPF-fixed sample, indicating that more cytosolic proteins are retained around the vesicles. This effect also explains the general lack of contrast in the glyoxal-fixed sample, in which vesicles are more difficult to detect. Superficially, images of glyoxal-fixed boutons resemble boutons fixed by high-pressure freezing more than they resemble PFA-fixed boutons: the demarcation of the vesicles is more difficult, due to the large amount of cytosolic proteins that are present around and between the vesicles. Scale bar $=200 \mathrm{~nm} . \mathrm{N}=1100-4900$ vesicles, from 2-4 independent experiments for each chemical fixation procedure and 782 vesicles for the HPF-fixation. 


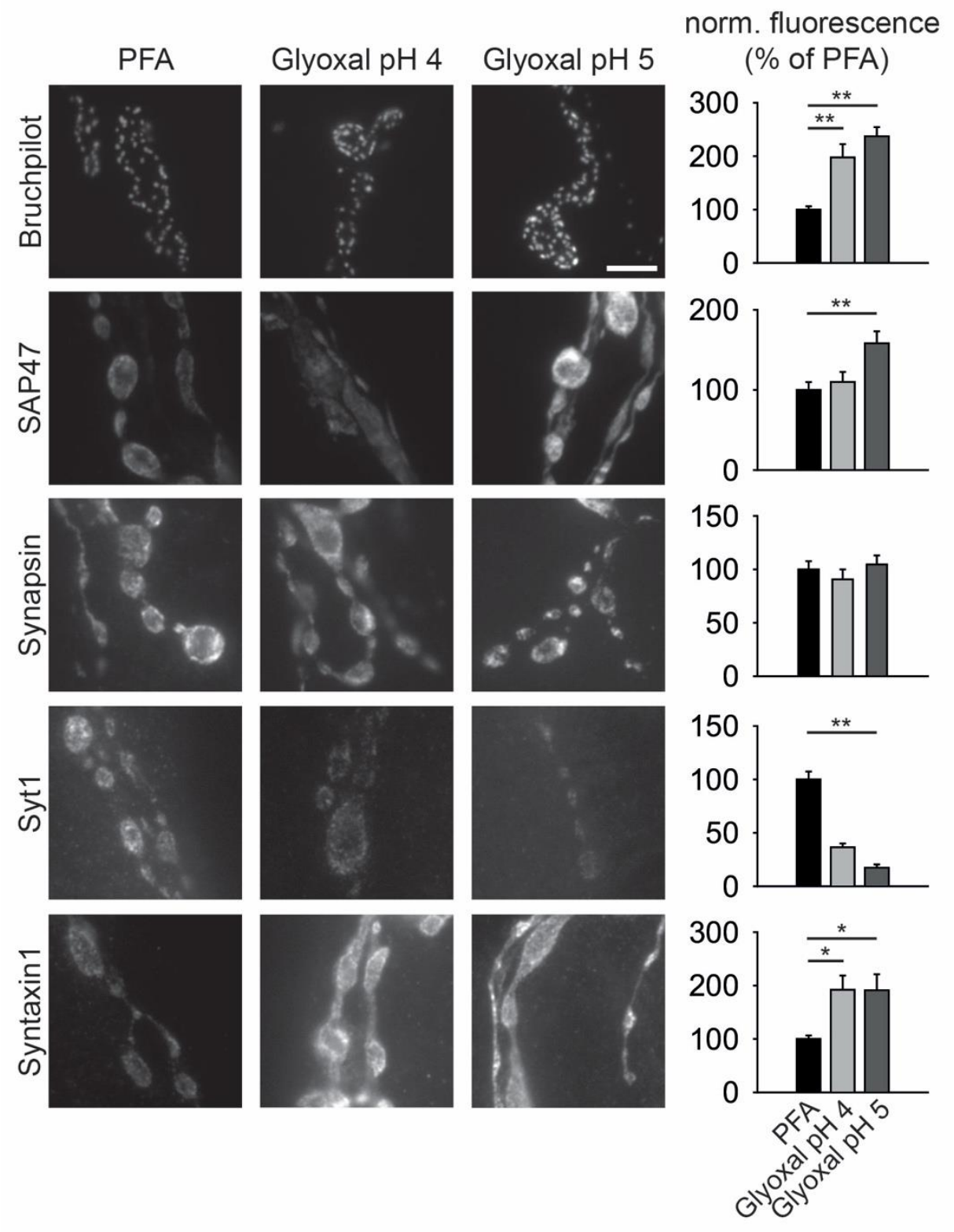

Appendix Figure S16 Glyoxal fixation in tissue from Drosophila larvae.

Fluorescence images of Drosophila neuromuscular junctions, immunostained for various neuronal proteins, are shown. The graphs indicate the average fluorescence intensity, corrected for background. Glyoxal $\mathrm{pH} 4$ and $\mathrm{pH} 5$ fixations provide significantly higher fluorescence signals, in comparison to PFA, for bruchpilot and syntaxin 1. Glyoxal pH 5 provides a significantly higher signal for SAP47. PFA fixation gives a significantly higher signal for synaptotagmin 1 (Syt1), in comparison to the glyoxal fixation. $\mathrm{N}=$ between 15 and 20 images analyzed per protein and fixation condition, in 2 independent experiments. Scale bar $=5 \mu \mathrm{m} .{ }^{*} p<0.05,{ }^{* *} p<0.01$. 

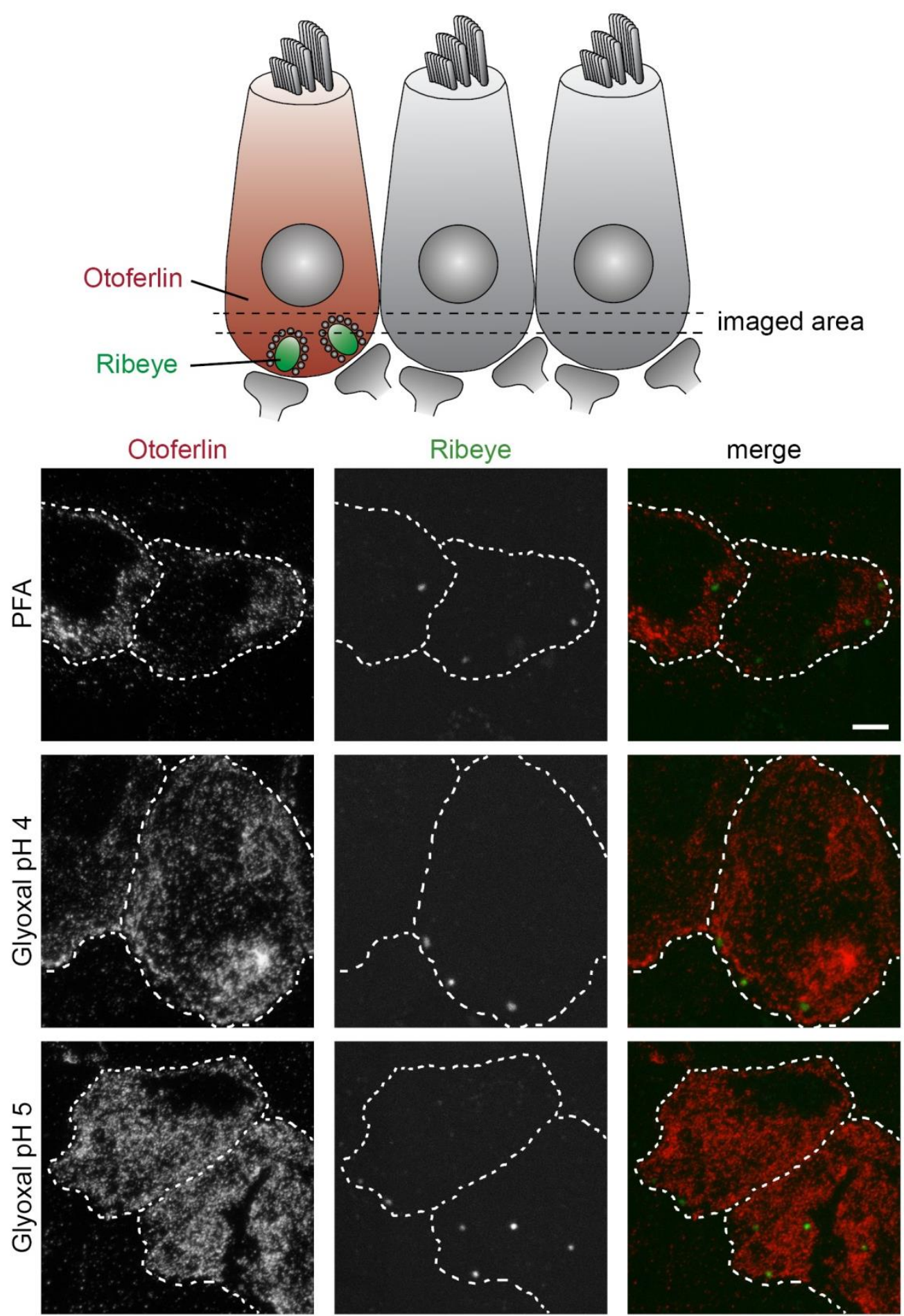

Appendix Figure S17 Glyoxal fixation in mammalian tissue: mouse inner hair cells.

Inner hair cells of the auditory system (Revelo et al, 2014) were immunostainined for the calcium sensor protein otoferlin (Atto647N, red), and for the ribbon synapse protein ribeye (Cy2, green). The overall morphology and antigenicity is preserved in glyoxal-fixed samples, at least as well as in PFAfixed samples. Scale bar $=2 \mu \mathrm{m}$. 

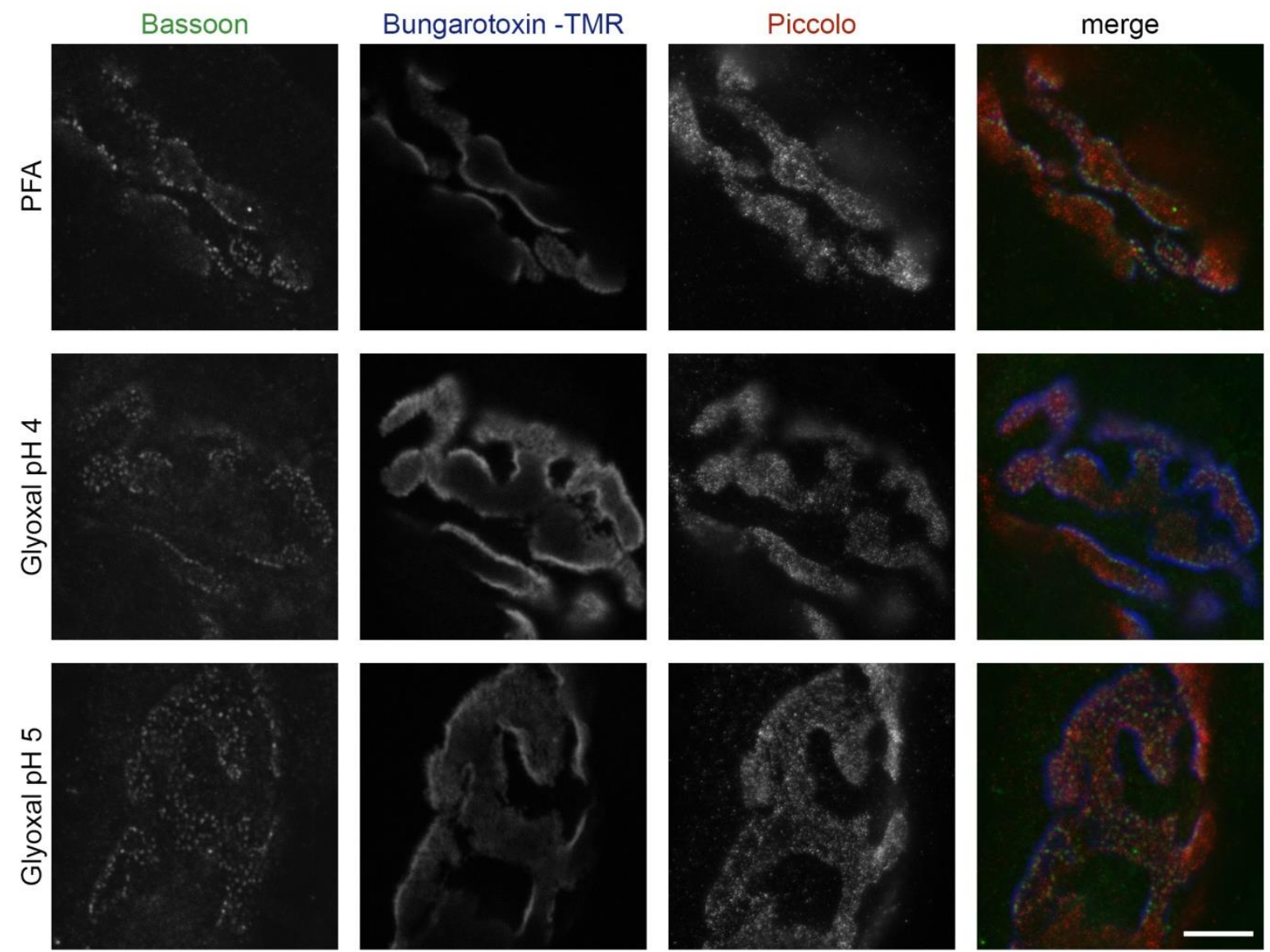

Appendix Figure S18 Glyoxal fixation in mammalian tissue: mouse neuromuscular junctions.

Synapses of the levator auris longus muscle were immunostained for bassoon (Cy2, green) and piccolo (Atto647N, red). Additionally, acetylcholine receptors were stained with tetramethylrhodamine-labeled bungarotoxin (blue). As in Supplementary Fig. 10, the overall morphology and antigenicity is preserved in glyoxal-fixed samples. Scale bar $=5 \mu \mathrm{m}$.

Appendix Table 1: Qualitative analysis of different fixation conditions. Hippocampal neurons were fixed for 1 hour with the fixatives indicated in the first column, and were then imaged in presence of $1.5 \mu \mathrm{M}$ FM 1-43, which reveals all membranes. Scale bar, $5 \mu \mathrm{m}$. + and - indicate the quality of the fixation regarding possible fragmentation and swelling

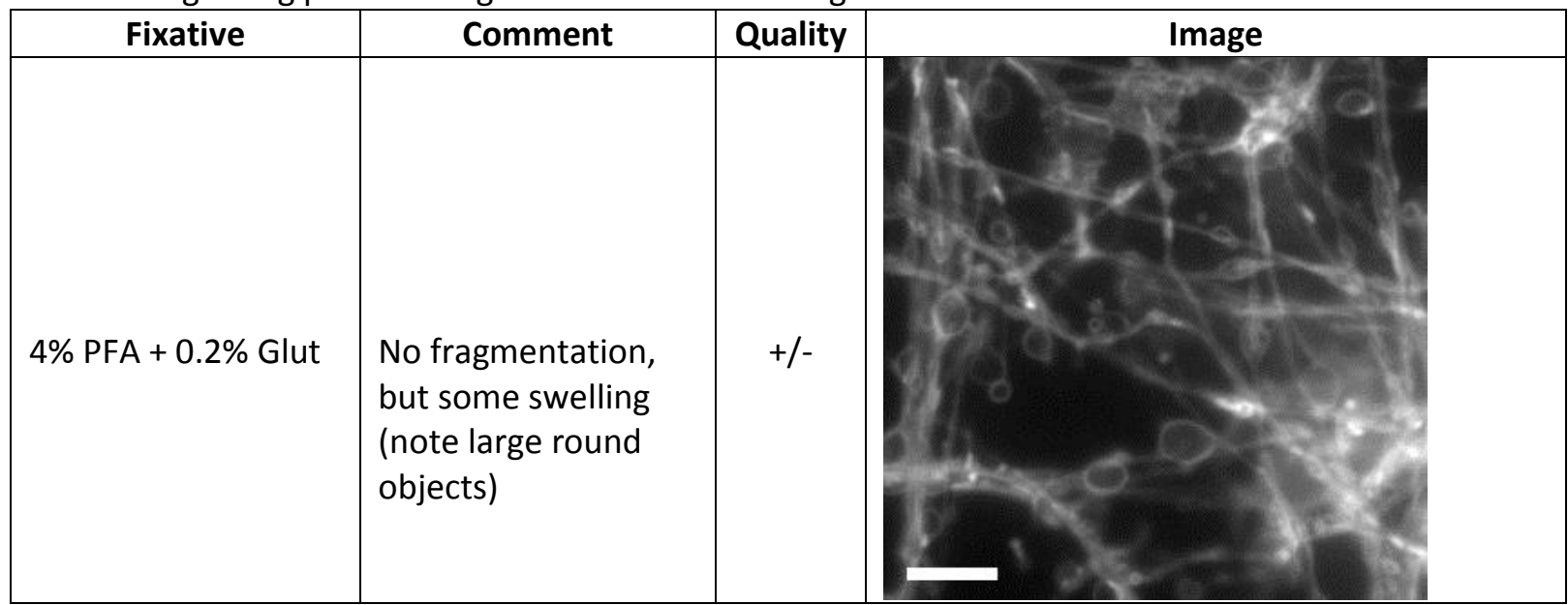




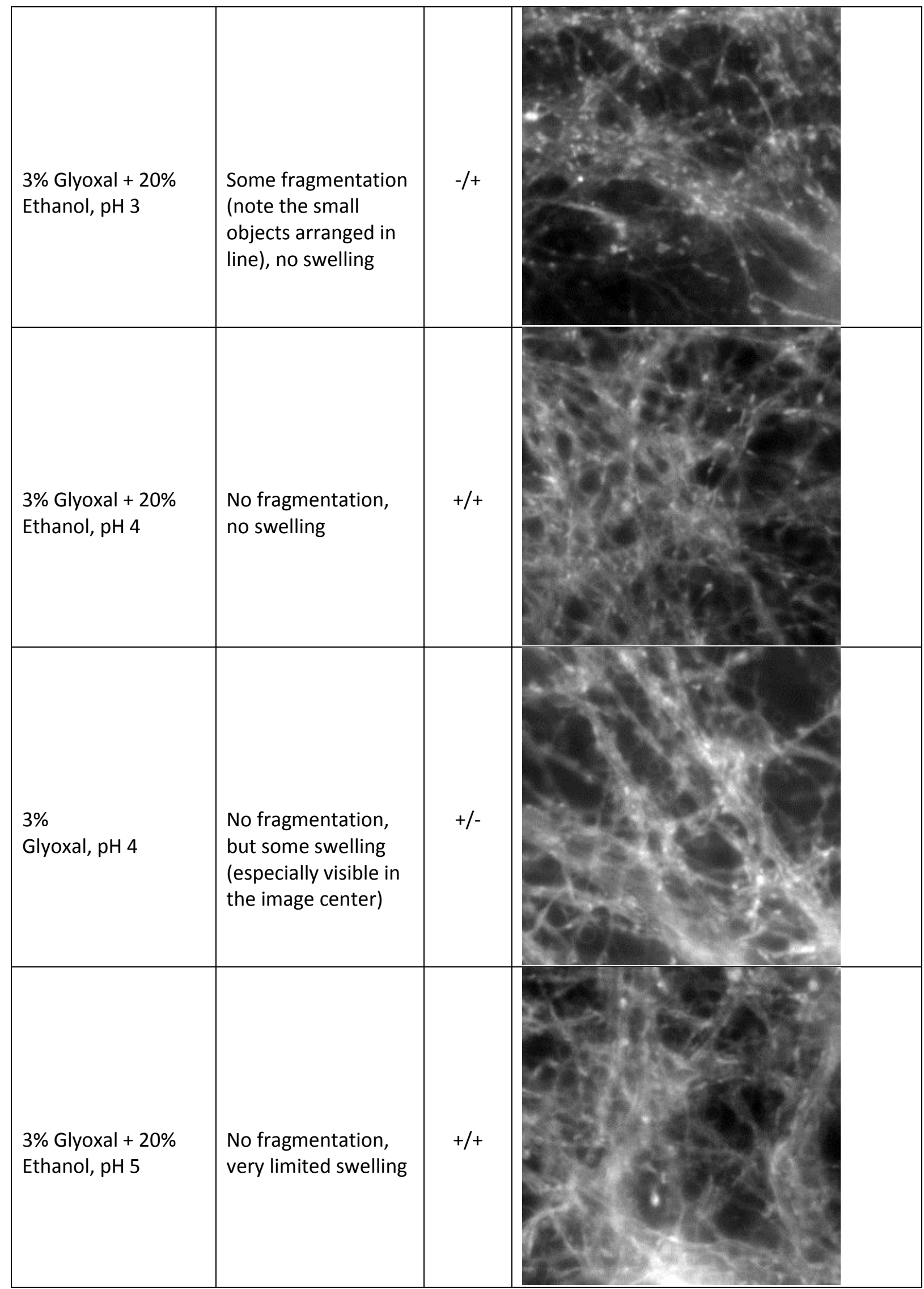




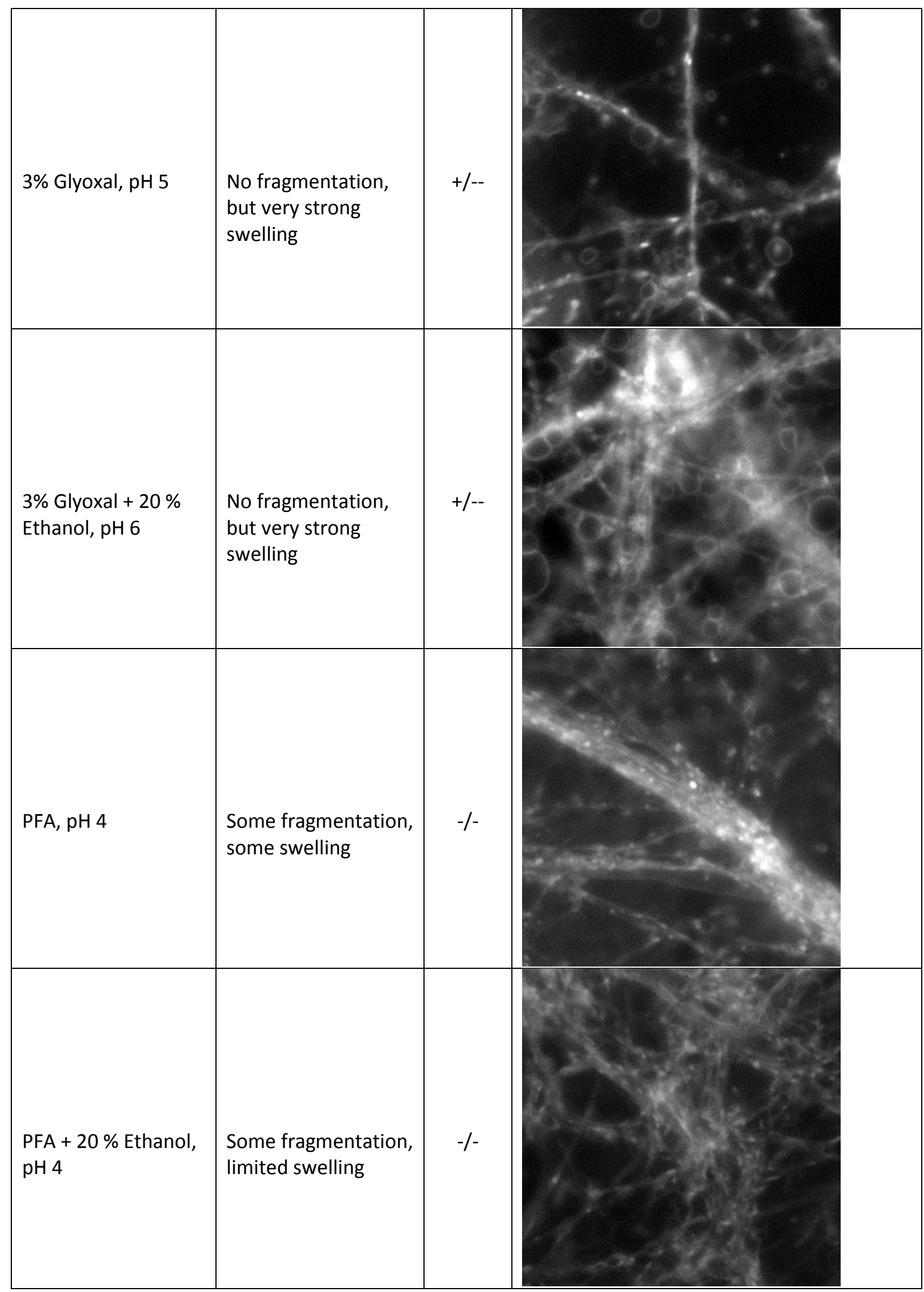




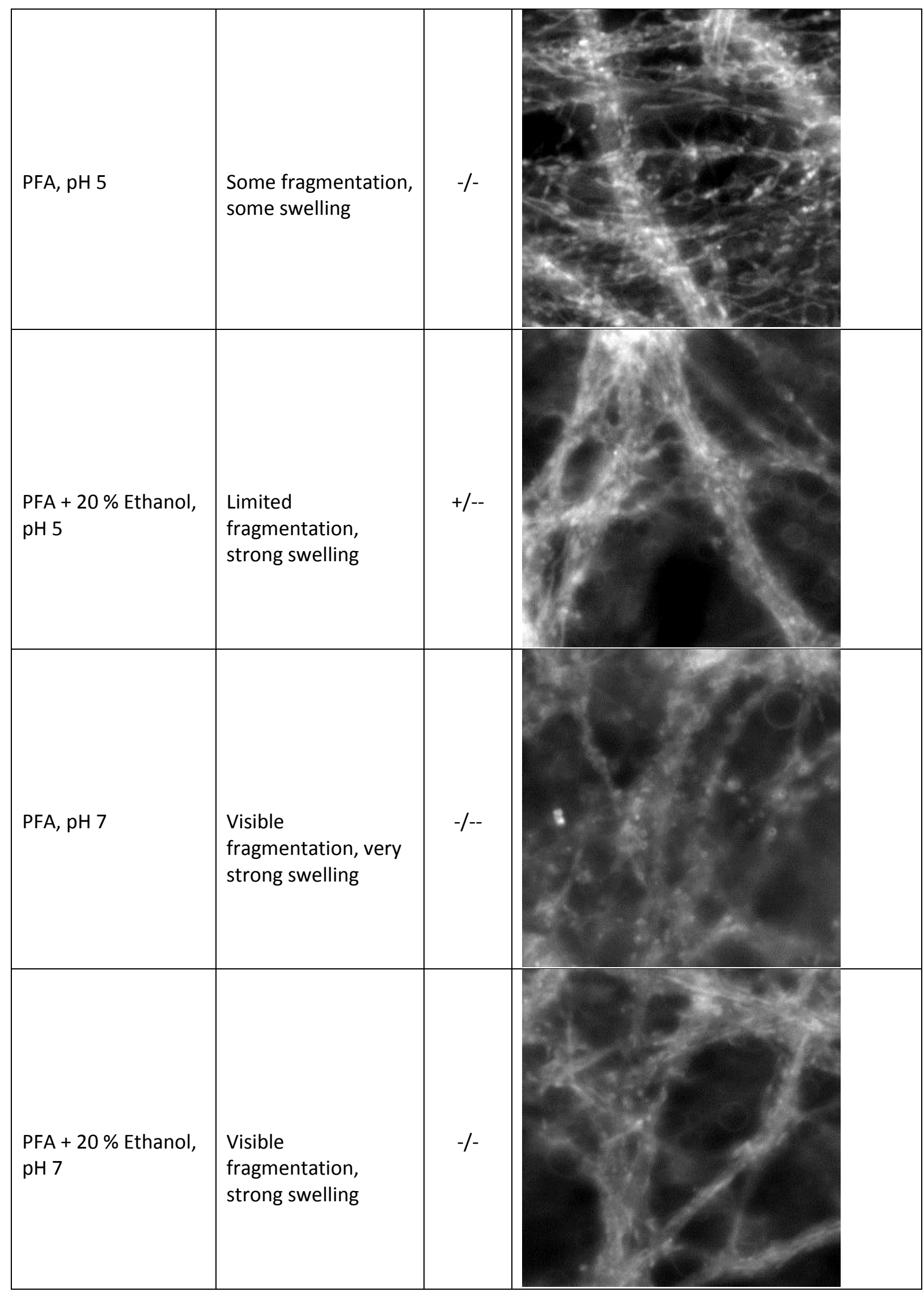




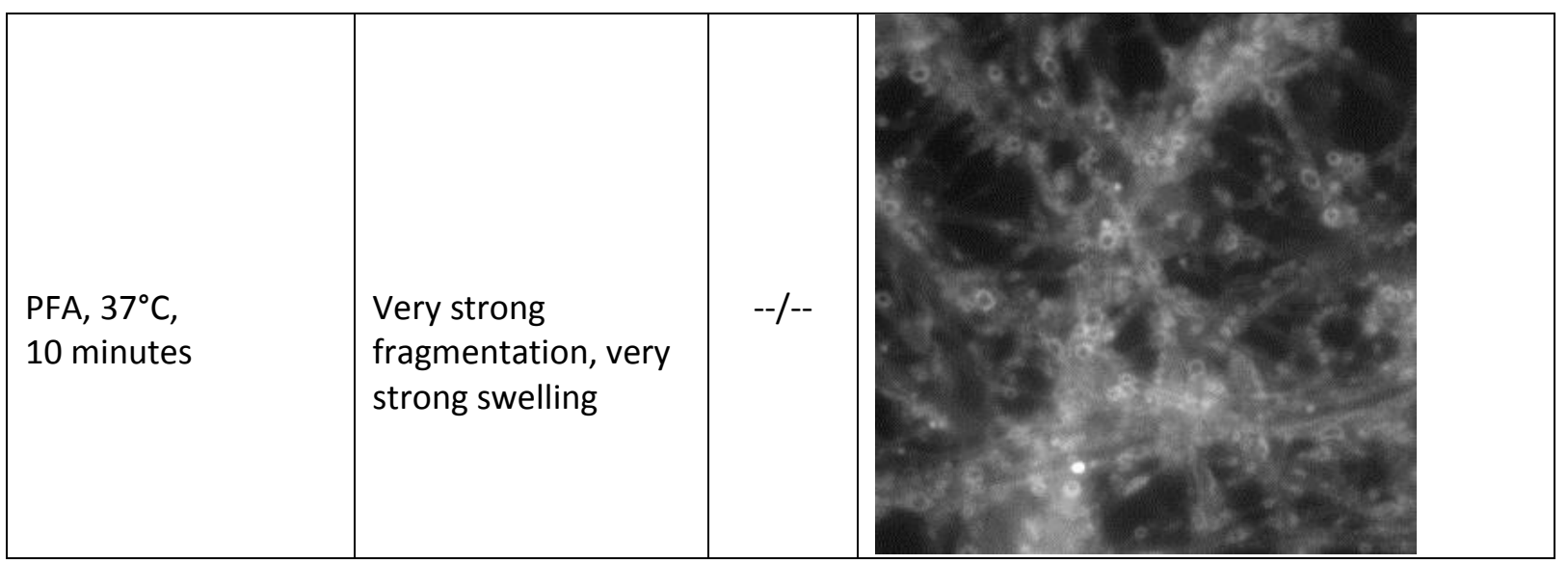

\section{Methods of collaborating laboratories}

\section{Ed Boyden}

\section{Immunocytoschemistry of HeLa cells (Figure 5)}

HeLa Cells were fixed with either 4\% PFA of glyoxal for $10 \mathrm{~min}$ at room temperature. Afterwards, cells were quenched for 5 min in PBS and $100 \mathrm{mM}$ glycine, following $10 \mathrm{~min}$ washing in PBS. Subsequently, the cells were incubated in the primary antibody (NUP160; abcam ab74147) for one hour at room temperature. The cells have been washed $20 \mathrm{~min}$ in PBS prior to the incubation in Atto647F coupled secondary antibody (Sigma Aldrich) for one hour at room temperature. Both antibody incubations have been done in blocking buffer ( $5 \%$ normal donkey serum, $0.25 \%$ Triton X-100 in PBS). After further washing in PBS for 20 min the cells were imaged with a spinning disk confocal microscope.

\section{Rory Duncan}

\section{Immunocytochemistry of AtT20 cells (Figure 6)}

For Syntaxin1, SNAP25 and LC3B immunostaining, AtT20 cells were cultured in duplicate on PDL-coated coverslips and fixed with 4\% PFA or glyoxal pH 5 by 60 minute incubation at room temperature. Autophagy was induced in cells intended for LC3B staining by 60 minute treatment with $160 \mathrm{nM}$ rapamycin immediately prior to fixation. Following fixation, cells were quenched with $50 \mathrm{mM} \mathrm{NH}_{4} \mathrm{Cl}$ for 20 min and permeabilized by 4 minute incubation in PBS supplemented with $0.5 \%(\mathrm{v} / \mathrm{v})$ Triton X-100. Epitope blocking was subsequently achieved by 15 minute incubation in blocking buffer $(0.5 \%$ (w/v) fish skin gelatin in PBS). Samples were immunolabeled for three hours at room temperature with the primary antibodies anti-Syx1 [HPC-1], anti-SNAP25 [SMI 81] or LC3B diluted in blocking buffer as detailed in Appendix Table 2. Cells were then washed for 30 mins in blocking buffer and incubated for 60 minutes in blocking buffer supplemented with 1:1000 Alexa 488 conjugated anti-rabbit IgG or antimouse IgG as appropriate. Samples were mounted with Mowiol after a further $30 \mathrm{~min}$ wash step. Samples were imaged on a Leica SP5 confocal laser scanning microscope using a HCX PL 
Apo 100X oil immersion objective lens. The pinhole was set to 1 Airy unit and images were acquired with a $1024 \times 1024$ pixel layout using a $0.04 \mu \mathrm{m}$ pixel size.

Appendix Table 2: Primary and secondary antibodies used for the immunostaining of AtT20 cells

\begin{tabular}{|c|c|c|c|}
\hline Primary antibody & Host species & Company & Dilution \\
\hline \hline Syx1 [HPC-1] & Mouse & Abcam ab3265 & $1: 1000$ \\
\hline SNAP25 [SMI 81] & Mouse & Abcam ab24737 & $1: 1000$ \\
\hline LC3B & Rabbit & Novus Biologicals NB600-1384 & $1: 500$ \\
\hline Secondary antibody & Host species & Company & Dilution \\
\hline \hline Anti-rabbit IgG (Alexa Fluor 488) & Goat & Fisher Scientific (10082502) & $1: 1000$ \\
\hline Anti-mouse IgG (Alexa Fluor 488) & Goat & Fisher Scientific (10328172) & $1: 1000$ \\
\hline
\end{tabular}

\section{Elisa D’Este/Stefan Hell}

\section{Neuronal cell culture and immunocytochemistry (Figure 7)}

Cultures of hippocampal neurons were prepared from Wistar rats of mixed sex at postnatal day P0-P1, in accordance with Animal Welfare Law of the Federal Republic of Germany (Tierschutzgesetz der Bundesrepublik Deutschland, TierSchG) and the Regulation about animals used in experiments (1st August 2013, Tierschutzversuchsverordnung). For the procedure of sacrificing rodents for subsequent preparation of any tissue, all regulations give in \$4 TierSchG are followed. Since sacrificing of animals is not an experiment on animals according to $\S 7$ Abs. 2 Satz 3 TierSchG, no specific authorization or notification is required. Cells were plated on coverslips coated with $100 \mu \mathrm{g} / \mathrm{ml}$ polyornithine (Sigma-Aldrich \#P3655) and $1 \mathrm{\mu g} / \mathrm{ml}$ laminin (BD Bioscience \#354232). Neuronal cultures were maintained in Neurobasal medium (Gibco \#21103049) supplemented with 2\% B27 serum-free supplement (Gibco \#17504044), 2 mM L-glutamine (Gibco \#25030) and penicillin/streptomycin (100 units $/ \mathrm{ml}$ and $100 \mu \mathrm{g} / \mathrm{ml}$, respectively, BiochromAG A2213). On the day after plating, $5 \mu \mathrm{M}$ cytosine $\beta$-D-arabinofuranoside (Sigma \#C1768) was added to the cultures.

For immunostainings, cells were washed in PBS and fixed for 20-25 min at room temperature with glyoxal pH 5 (except for staining of neurofilament and spectrin $\mathrm{pH} 4$ was used) or PFA or PFA with $0.2 \%$ glutaraldehyde in PHEM buffer (60 mM PIPES, 25 mM HEPES, 10 mM EGTA, 2 $\mathrm{mM} \mathrm{MgCl} 2 ; \mathrm{pH}$ 6.9). Cells were quenched with ammonium chloride and glycine $(100 \mathrm{mM}$ each) for $5 \mathrm{~min}$, were permeabilized with $0.1 \%$ Triton X-100 for another $5 \mathrm{~min}$, and blocked in PBS supplemented with $1 \%$ BSA for 30 min. Both primary and secondary antibody incubations were performed in PBS for 1 hour at room temperature. Phalloidin incubation was performed together with secondary antibodies. Samples were mounted in Mowiol supplemented with DABCO. 


\section{Reagents and antibodies}

The antibodies and reagents used are: anti-betalll tubulin (Tuj1) (Biolegend, 1:400 dilution), anti-betall spectrin (BD Biosciences, 1:200 dilution); anti-Ankyrin G (SantaCruz, 1:50); antineurofilament L (Synaptic Systems, 1:400); anti-pan-Nav (Sigma-Aldrich, 1:100); anti-Kv7.2 (Synaptic Systems, 1:200); phalloidin-STAR635 (Abberior). Sheep anti-mouse (Dianova) and goat anti-rabbit (Dianova) were labeled with STAR635P or START580 (Abberior)

Imaging was performed on a two-color Abberior STED 775 QUAD scanning microscope (Abberior Instruments $\mathrm{GmbH}$, Göttingen, Germany) equipped with $561 \mathrm{~nm}$ and $640 \mathrm{~nm}$ pulsed excitation lasers, a pulsed $775 \mathrm{~nm}$ STED laser, and a 100x oil immersion objective lens (NA 1.4).

\section{Marcel Lauterbach}

\section{Immunhistochemistry of Sepia officinalis skin samples (Figure 8)}

The experiment was conducted on adult male Sepia officinalis with a mantle length of $10 \mathrm{~cm}$. Animals were reared in a closed seawater system with salinity of $33 \%$ at $20^{\circ} \mathrm{C}$. To harvest skin samples, animals were anesthetized in an isotonic solution prepared by mixing a $7.5 \%$ (w/v) $\mathrm{MgCl}_{2} * 6 \mathrm{H}_{2} \mathrm{O}$ in deionized water solution with an equal volume of seawater. Animals were then decapitated and samples of dorsal skin and fin were excised and fixed overnight at $4^{\circ} \mathrm{C}$ in either $3 \%$ glyoxal pH 5 or $4 \%$ PFA. The following procedures were the same for both fixation techniques and performed in parallel. After cryoprotection in $30 \%(\mathrm{w} / \mathrm{v})$ sucrose in PBS for 2 days at $4^{\circ} \mathrm{C}$ the tissue was embedded in Tissue-Tek O.C.T. (Sakura Finetec Europe B. V., Alphen aan den Rijn, The Netherlands) and sectioned at $100 \mu \mathrm{m}$ thickness.

Prior to permeabilization in acetone for $15 \mathrm{~min}$ at $-20^{\circ} \mathrm{C}$, the sections were washed twice for 10 min in PBS. Samples were blocked for 2 hours at room temperature in a freshly prepared solution containing 2\% Normal Goat Serum, 1\% Bovine Serum Albumin, 0.2\% Triton X-100, $0.1 \%$ cold water fish gelatin, $0.3 \mathrm{M}$ glycine and $0.05 \%$ thimerosal. The samples were incubated for 18 hours at $4{ }^{\circ} \mathrm{C}$ with the primary antibody diluted in blocking solution. The antibody used was a rabbit polyclonal antibody against FMRFamide (1:500, ImmunoStar Inc, Hudson, Wisconsin, USA, Cat. No. 20091, RRID: AB_572232). After 6 × 10 min washing in PBS containing $0.2 \%$ Tween 20 (PBST) the samples were incubated with the secondary antibody (Alexa 488 anti-rabbit (Thermo Fisher Scientific, Waltham, Massachusetts, USA, Cat. No. A11008). The sections were washed $6 \times 15 \mathrm{~min}$ in PBST and mounted in Fluorescence Mounting Medium (Dako, Agilent Technologies, Santa Clara, California, USA). Confocal fluorescence images were recorded at an LSM 880 microscope (Zeiss, Oberkochen, Germany) with a $63 x$ NA 1.2 Oil immersion objective 


\section{Stephan E. Lehnart}

\section{Isolation and immunocytochemistry of murine ventricular myocytes (Figure 9)}

Ventricular myocytes (VM) of 12 week old C57BI/6N mice were isolated according to previously published protocols (Wagner et al, 2012, 2014). In short, hearts were attached to a modified Langendorff perfusion system and digested by perfusion through the aorta using collagenase type II (Worthington).

Freshly isolated VM were immediately plated on laminin-coated coverslips. After $30 \mathrm{~min}$ of plating, VM were either fixed with 4\% PFA, pH 7.4 or with $3 \%$ glyoxal, pH 5 for 10 min at room temperature. After fixation, VM were washed with blocking buffer $(0.2 \%$ Triton X-100, $10 \%$ bovine calf serum in PBS) once. Primary antibodies were diluted in blocking buffer and VM were incubated with the appropriate antibody dilution (Appendix Table 3) over night at $4^{\circ} \mathrm{C}$. Afterwards, VM were washed with blocking buffer three times, secondary antibodies were diluted in blocking buffer and VM were incubated with the appropriate secondary antibody dilution (Appendix Table 3 ) over night at $4^{\circ} \mathrm{C}$. After three washing steps with PBS, pH 7.4, VM were mounted using ProLong Gold Antifade Mountant (molecular probes via Thermo Fisher).

Immunostained murine VM were imaged using a TCS SP8 STED microscope (Leica) and a 100x oil objective (1.4 NA). Abberior STAR 635P was exited at a wavelength of $635 \mathrm{~nm}$ and fluorescence was detected between $650 \mathrm{~nm}$ and $700 \mathrm{~nm}$. STED imaging was performed with a $775 \mathrm{~nm}$ depletion laser and gating between $0.5 \mathrm{~ns}$ and $6 \mathrm{~ns}$. All images were acquired with a pixel size of $23 \mathrm{~nm} \times 23 \mathrm{~nm}$ and a scanning speed of $600 \mathrm{~Hz}$ (pixel dwell time $0.4 \mu \mathrm{s}$ ).

Appendix Table 3: Antibodies used for immunostaining of murine VM

\begin{tabular}{|c|c|c|c|}
\hline Primary antibody & Host species & Company & Dilution \\
\hline \hline caveolin-3 & rabbit & Abcam (\#ab2912) & $1: 500$ \\
\hline ryanodine receptor type 2 & mouse & Thermo Fisher (\#Ma3-916) & $1: 500$ \\
\hline Secondary antibody & Host species & Company & Dilution \\
\hline \hline anti-rabbit Abberior STAR635P & goat & Abberior (\#2-0012-007-2) & $1: 1000$ \\
\hline anti-mouse Abberior STAR635P & goat & Abberior (\#2-0002-007-5) & $1: 1000$ \\
\hline
\end{tabular}

\section{Tobias Moser}

\section{Immunohistochemistry of auditory inner hair cells (Figure 10)}

Acutely dissected organs of Corti from hearing p14-p16 mice were fixed (either 10 min in icecold fixative or $1 \mathrm{~h}$ on ice) using either 4\% PFA or glyoxal at $\mathrm{pH} 5.0$, and were then permeabilized and blocked with goat serum dilution buffer (GSDB) containing $16 \%$ normal goat serum, $450 \mathrm{mM} \mathrm{NaCl}, 0.3 \%$ Triton $\mathrm{X}-100$ and $20 \mathrm{mM}$ phosphate buffer at $\mathrm{pH} 7.4$. Subsequently, the following primary antibodies were applied in GSDB over night at $4^{\circ} \mathrm{C}$ : mouse monoclonal anti-otoferlin (Abcam; ab\#53233), rabbit polyclonal anti-calretinin (Swant; \#7697), mouse monoclonal CtBP2 (BD Bioscience; 612044), CaV1.3 (Alomone; ACC005), Homer1 (Synaptic Systems; 160 002) and mouse monoclonal anti-PSD95 (clone 7E3- 
1B8; Sigma Aldrich; P246). After extensive washing in PBS, goat anti-mouse Abberior STAR580 and goat anti-rabbit Abberior STAR635p fluorophore-conjugated secondary antibodies (Abberior 2-0002-005-1 and Abberior 2-0012-007-2, respectively) were used for visualization. Finally, immunolabeled organs of Corti were washed repeatedly in PBS prior to mounting with Mowiol. In all cases, the dissections, fixations and immunostaining procedures were performed in parallel and specimens were subsequently imaged during the same imaging session.

Image acquisition was performed in confocal mode on an Abberior Instruments Expert Line STED microscope (based on an Olympus IX83 inverted microscope) running Imspector software, with excitation lasers at 561 and $640 \mathrm{~nm}$ and a 1.4 NA UPlanSApo 100x oil immersion objective. Image stacks were acquired with xy pixel sizes of $60 \times 60 \mathrm{~nm}$ and a zstep size of $200 \mathrm{~nm}$. The image stacks were summed to generate 2D projection images, before analyzing the total staining intensities.

\section{Tiago Outeiro}

\section{Cell culture and Transfection}

Human neuroglioma cells (H4) were cultured in Opti-MEM I with Glutamax (Life Technologies- Gibco, Carlsbad, CA, USA) supplemented with 10\% Fetal Bovine Serum Gold (PAA, Cölbe, Germany) and $1 \%$ Penicillin-Streptomycin (PAN, Aidenbach, Germany), at $37^{\circ} \mathrm{C}$, with $5 \% \mathrm{CO}_{2}$. $24 \mathrm{~h}$ prior to transfection, $\mathrm{H} 4$ cells were plated in 12 -well plates (Costar, Corning, New York, USA). On the subsequent day, the cells were transfected with the SynT and Synphilin-1 constructs, to induce the formation of aSyn assemblies, or with the Vimentin- $\mathrm{O} 2$ construct, using the calcium phosphate method. Briefly, a mix of DNA, $\mathrm{H}_{2} \mathrm{O}$ and $2.5 \mathrm{M}$ calcium chloride was added dropwise to $2 \times \mathrm{BES}$-buffered saline solution (50 mM BES, $280 \mathrm{mM} \mathrm{NaCl}, 1.5 \mathrm{mM} \mathrm{Na}_{2} \mathrm{HPO}_{4} \mathrm{XH}_{2} \mathrm{O}, \mathrm{pH}$ 6.98). The cells were incubated with plasmidcalcium-phosphate coprecipitates for $17 \mathrm{~h}$, after which the media were exchanged, and the cells were allowed to recover for $24 \mathrm{~h}$ before fixation.

\section{Immunocytochemistry (Figure 11)}

Cells were fixed with $4 \%$ paraformaldehyde or with $3 \%$ glyoxal $(\mathrm{pH} 5)$ at room temperature (RT) for 10 minutes. The cells were afterwards permeabilized with $0.1 \%$ Triton X-100 (SigmaAldrich, St. Louis, MO, USA). The cells transfected with Vimentin-O2 were stained with DAPI and imaged. For the SynT+ Synphilin-1 model, cells were blocked in 1.5\% normal goat serum (PAA, Cölbe, Germany)/1xPBS (1.37 M NaCl, $27 \mathrm{mM} \mathrm{KCl,} 101.4 \mathrm{mM} \mathrm{Na} \mathrm{HPO}_{4} 7 . \mathrm{H}_{2} \mathrm{O}$, $\left.16.7 \mathrm{mM} \mathrm{KH}_{2} \mathrm{PO}_{4}\right)$, and were then incubated with primary antibody: Syn1 (1:1000, BD Transduction Laboratory, New Jersey, USA) or Vimentin (1:1000, SigmaAldrich, St. Louis, MO, USA) for 3h, and with secondary antibody (Alexa Fluor 555 goat anti rabbit IgG, (Life Technologies- Invitrogen, Carlsbad, CA, USA)) for 30min at RT. Cells were finally stained with DAPI (Life Technologies- Invitrogen, Carlsbad, CA, USA) (1:5000 in DPBS) for 5 min, and were maintained in 1xPBS for imaging. 


\section{Imaging with an inverted epifluorescence Olympus IX 71 microscope}

All samples were imaged using an Olympus IX 71 epifluorescence microscope, equipped with a $100 \mathrm{~W}$ mercury lamp and a F-View II CCD camera (Soft Imaging Systems GmbH). A 60X UPlanApo oil immersion objective (NA 1.35) from Olympus was used. Excitation and emission filter with 562/585 $\mathrm{nm}$ was used and image acquisition was performed using the Olympus Cell^P software.

\section{Peter Rehling}

\section{Immunocytochemistry of U2OS and HeLa cells (Figure 12)}

Cells were grown on coverslips and MitoTracker ${ }^{\mathrm{TM}}$ Orange CMTMRos (Thermofisher, M7510) was applied for 20 minutes at $37^{\circ} \mathrm{C}$. Cells were washed briefly in PBS and fixed in $4 \%$ paraformaldehyde (AppliChem $\mathrm{A} 3813,0500$ ) or $3 \%$ glyoxal for $20 \mathrm{~min}$ at $37^{\circ} \mathrm{C}$. Cells were washed with PBS, permeabilized with $0.2 \%$ Triton-X100 (Roth, 3051.3) for 20 min, washed again and blocked in blocking buffer (1\% bovine serum albumin in PBS) for 20 min at room temperature. Subsequently, cells were incubated in home-made primary rabbit antibodies anti-ATP5B, anti-TIM23, anti-COA6 and anti-NDUFA9, diluted 1:200 in PBS for 60 minutes. Following washing in PBS, cells were incubated with secondary antibodies (Alexa Fluor 488 goat anti-rabbit IgG, ThermoFisher Scientific, A-11001) for $30 \mathrm{~min}$. Final washing was followed by mounting in histology mounting medium containing DAPI (Fluoroshield ${ }^{\mathrm{Tm}}$; SigmaAldrich, F6057). Images were taken with a Leica TCS SP5 confocal microscope using a 100X HCX PL APO oil immersion STED objective (NA 1.4). Images were acquired using the Leica LAS AF imaging software, with a pixel size of $60 \times 60 \mathrm{~nm}$ and a scanning speed of $1000 \mathrm{~Hz}$.

\section{Blanche Schwappach}

\section{Immunocytochemistry of HeLa P4 cells (Figure 13)}

HeLa P4 cells (Charneau P, Mirambeau G, Roux P, Paulous S, Buc H, 1994), were obtained from the NIH AIDS Reagent Program and were grown in DMEM (Gibco \#41966-029) supplemented with $10 \%$ (v/v) FBS (Biochrom \#S0615) and 2 mM L-glutamine (Gibco \#25030024) under $5 \% \mathrm{CO}_{2}$ at $37^{\circ} \mathrm{C}$. They were tested for contamination by mycoplasma on a regular basis.

For the glucocorticoid receptor stimulation (employed for the glucocorticoid receptor stainings) cells were treated with the corresponding volume of solvent (absolute ethanol) or a stock resulting in a final concentration of $100 \mathrm{nM}$ of dexamethasone (Sigma \#D4902) in DMEM for $60 \mathrm{~min}$ at $37^{\circ} \mathrm{C}$.

For immunofluorescence, cells were fixed with 4\% (w/v) PFA (PanReac AppliChem \#A3813) in PBS or $3 \% \mathrm{w} / \mathrm{v}$ glyoxal $\mathrm{pH} 5$ solution, for $15 \mathrm{~min}$ (cells stained for SGTA and EEA1) or 60 min and then permeabilized with $0.3 \%$ Triton X-100/0.05\% SDS in PBS for 10 min at room temperature. Samples were blocked with $10 \%$ FBS in PBS for $30 \mathrm{~min}$ and incubated with primary antibodies (see Appendix Table 4) diluted in blocking buffer overnight at $4^{\circ} \mathrm{C}$. 
Incubation with Alexa Fluor secondary antibodies (Invitrogen) was performed for 60 min at room temperature. The samples were mounted with Mowiol-DAPI for the confocal microscope or incubated with DAPI ( $1 \mu \mathrm{g} / \mathrm{mL}$, Sigma-Aldrich \#D9542) in PBS for $10 \mathrm{~min}$ for the screening microscope.

Appendix Table 4: primary antibodies used for immunocytochemistry

\begin{tabular}{|c|c|c|c|}
\hline primary antibody & host species & company & dilution \\
\hline \hline BAG6 & rabbit & custom-made & $1: 300$ \\
\hline APPL1 & rabbit & Cell Signaling; 3858 & $1: 200$ \\
\hline ATPB & mouse & abcam; ab14730 & $1: 400$ \\
\hline Calnexin & mouse & BD transduction; 610547 & $1: 200$ \\
\hline Caveolin-1 & rabbit & Cell Signaling; 3267 & $1: 200$ \\
\hline EEA1 & mouse & BD transduction; 610456 & $1: 200$ \\
\hline Emerin & rabbit & SC; sc-15378 & $1: 150$ \\
\hline GATA-6 & rabbit & Cell Signaling; 5851 & $1: 200$ \\
\hline GM130 & mouse & BD transduction; 610823 & $1: 300$ \\
\hline GR & rabbit & SC; sc-8992 & $1: 250$ \\
\hline GS28 & mouse & BD transduction; 611184 & $1: 200$ \\
\hline Hsc70 & mouse & StressMarq; SMC-151 & $1: 200$ \\
\hline LAMP1 & mouse & DHSB; H4A3 & $1: 400$ \\
\hline LC3B & rabbit & abcam; ab48394 & $1: 200$ \\
\hline Nucleoporin p62 & mouse & BD transduction; 610497 & $1: 200$ \\
\hline Sec61ß & rabbit & custom-made; Berhard Dobberstein, & \multirow{2}{*}{$1: 300$} \\
\hline SGTA & chicken & Heidelberg & $1: 400$ \\
\hline Stx5 & rabbit & custom-made & $1: 200$ \\
\hline host species & SySy; 110053 & dilution \\
\hline secondary antibody & goat & Invitrogen, A11034 & $1: 1000$ \\
\hline anti-Rabbit IgG (Alexa Fluor 488) & Invitrogen, A21235 & $1: 1000$ \\
\hline anti-Mouse IgG (Alexa Fluor 647) & goat & Invitrogen, A21449 & $1: 1000$ \\
\hline anti-Chicken IgY (Alexa Fluor 647) & goat & & \\
\hline
\end{tabular}

\section{Imaging with a LSM 510-META confocal microscope (Zeiss)}

HeLa P4 cells were analyzed using an Axiovert 200M fluorescence microscope with a 63x Plan-Neofluar 1.3 NA water-corrected objective and appropriate filter settings. Images were taken using a LSM 510-META confocal laser scanning microscope (Zeiss). For confocal imaging a UV laser (405 nm), a tunable Argon laser (488 nm) and HeNe laser lines (633 nm) were used for excitation.

\section{Ilaria Testa}

Immunohistochemistry of U2OS cells and primary hippocampal neurons (Figure 14) U2OS cells were cultured in DMEM, supplemented with $1 \%$ penicillin/streptomycin and $10 \%$ fetal bovine serum, and seeded on $18 \mathrm{~mm}$ coverslips. 
Primary hippocampal cultures were prepared from E18 Sprague Dawley rat embryos and cultured on poly-ornithine coated $18 \mathrm{~mm}$ coverslips, under normal conditions. The animal experiments were approved by the Institutional animal care and use committee of the Karolinska Institutet.

For the imaging of endoplasmic reticulum, U2OS cells were transfected with an EGFP-KDEL plasmid using Lipofectamine LTX with Plus reagent (ThermoFisher Scientific), following the instruction manual from the company. $24 \mathrm{~h}$ after transfection the cells were fixed with either PFA $4 \%$ or glyoxal $3 \%$ solution ( $\mathrm{pH} 5$ ), following the protocol described above (30min on ice + $30 \mathrm{~min}$ at RT). Afterwards, the cells were quenched with $100 \mathrm{mM} \mathrm{NH}_{4} \mathrm{Cl}$ for $20 \mathrm{~min}$; permeabilization and blocking was done for $15 \mathrm{~min}$ in $2.5 \% \mathrm{BSA}$ and $0.1 \%$ Triton X-100 in PBS. The cells were then incubated for $60 \mathrm{~min}$ with the primary antibodies: Rabbit polyclonal against GFP (1:100, Abcam, ab6556) or Tom20 Antibody (1:100, Santa Cruz Biotechnology, sc-11415). After washing in blocking solution for $15 \mathrm{~min}$, the cells were incubated with the secondary antibody for $60 \mathrm{~min}$ : Star Red (1:200, Abberior). Subsequent washing in phosphate buffer saline was followed by embedding in Mowiol.

Primary hippocampal neurons after 2 days of culture were fixed with either PFA $4 \%$ or glyoxal $3 \%$ solution ( $\mathrm{pH} 5$ ), following the protocol described above (60 min at RT), and were subsequently quenched for $30 \mathrm{~min}$ in $100 \mathrm{mM} \mathrm{NH}_{4} \mathrm{Cl}$. Permeabilization was achieved by incubating the neurons for $15 \mathrm{~min}$ in blocking solution, containing $2.5 \% \mathrm{BSA}$ and $0.1 \%$ Triton X-100 in PBS. The samples were then incubated with the Sodium / Potassium ATPase alpha-3 Antibody (ThermoFisherScientific, MA3-915) for 60 min at RT. After washing 15 min in blocking solution, Star Red secondary antibody (Abberior) was applied for $60 \mathrm{~min}$, at room temperature. Subsequent washing in high salt PBS $(500 \mathrm{mM} \mathrm{NaCl})$ and PBS was followed by embedding in Mowiol.

\section{Imaging with a custom STED/confocal}

The confocal images of KDEL, Tom20 and Sodium / Potassium ATPase alpha-3 were recorded with a custom built STED/confocal microscope equipped with a 100X/1.4 oil objective, using a $640 \mathrm{~nm}$ laser line to excite STAR RED and a $775 \mathrm{~nm}$ laser to deplete the signal.

\section{Bolek Zapiec}

\section{Mice}

Mouse experiments were performed in accordance with the German Animal Welfare Act, European Communities Council Directive 2010/63/EU, and the institutional ethical and animal welfare guidelines of the Max Planck Research Unit for Neurogenetics. Approval came from the Regierungspräsidium Darmstadt and the Veterinäramt of the City of Frankfurt. C57BL6/J female littermates at 3 weeks were anesthetized with an intraperitoneal injection of ketamine/xylazin sodium chloride $(\mathrm{NaCl})$ solution $(210 \mathrm{mg} / \mathrm{kg}$ ketamine, 10 $\mathrm{mg} / \mathrm{kg}$ xylazin). Mice were perfused with $12 \mathrm{ml} \mathrm{0.9 \%} \mathrm{NaCl}$ solution, and then with either 24 $\mathrm{ml}$ 4\% paraformaldehyde (PFA) in Phosphate Buffer Saline (PBS) or $24 \mathrm{ml} 3 \% \mathrm{pH} 4$ glyoxal fixative solution, both freshly made just prior to use. The olfactory mucosa and olfactory 
bulbs were dissected and post-fixed overnight at $4^{\circ} \mathrm{C}$ before washing in PBS 3 times for 10 minutes, decalcifying in $450 \mathrm{mM}$ EDTA in PBS at $4^{\circ} \mathrm{C}$ overnight, incubating in $15 \%$ sucrose in PBS at $4^{\circ} \mathrm{C}$ for $4 \mathrm{~h}$, in $30 \%$ sucrose in PBS at $4^{\circ} \mathrm{C}$ overnight, and embedding in OCT medium (TissueTek).

\section{Immunohistochemistry (Figure 15)}

Frozen blocks of mouse olfactory tissue were sectioned at $12 \mu \mathrm{m}$ thickness and collected on SuperFrost Ultra Plus slides (ThermoFisher). The slides were washed 3 times for 5 min with PBS at RT, then they were blocked in 10\% NDS in 0.2\% Triton X-100 PBS (PBST) at RT for $2 \mathrm{~h}$. The blocking solution was tipped off the slides, and the sections were incubated in a primary antibody solution containing $3 \%$ NDS in PBST overnight at $4^{\circ} \mathrm{C}$. Slides were then washed 3 times in PBST for $10 \mathrm{~min}$ at RT and incubated in a secondary antibody solution containing $3 \%$ NDS in PBS. The slides were subsequently washed 3 times in PBS for 10 min each and stained with DAPI at 1:10,000 in PBS for 10 minutes before being washed 3 times for 5 minutes in PBS. The primary antibodies (1:500) were goat anti-OMP (Wako, 544-10001-WAKO), mouse monoclonal anti-ß3-tubulin (Sigma T8660), rabbit monoclonal anti-neuropilin-1 (Abcam, AB81321), goat anti-neuropilin-2 (R\&D Systems, AF567), guinea pig anti-VGLUT2 (Synaptic Systems, 135 404), and rabbit anti-VGLUT2 (Synaptic Systems, 135 403). The secondary antibodies (1:500) were donkey anti-goat Alexa488 (ThermoFisher, A11055), donkey antirabbit Alexa546 (ThermoFisher, A10040), and donkey anti-mouse Cy5 (Jackson ImmunoResearch, 715-175-150). The slides were then imaged on a Zeiss LSM 710 confocal microscope. 
04 | Comparative synaptosome imaging: a semi-quantitative method to obtain copy numbers for synaptic and neuronal proteins

Katharina N. Richter, Hanna Wildhagen, Martin S. Helm, Jan-Eike Ußling, Thomas Schikorski \& Silvio O. Rizzoli

Sci Rep. 2018 Oct 4; 8(1):14838

https://www.nature.com/articles/s41598-018-33130-6

doi: 10.1038/s41598-018-33130-6

Author contribution of Katharina N. Richter:

- Design (together with Silvio O. Rizzoli) and performance of the initial immunostaining experiments (Figure 1, 2, 3)

- Preparation of the manuscript together with Silvio O. Rizzoli (and comments from all other authors)

This article has been reproduced with permission from Springer Nature Ltd. under open access copyright and licensing regulations (https://creativecommons.org/licenses/by/4.0/legalcode). 


\section{Comparative synaptosome imaging: a semi-quantitative method to obtain copy numbers for synaptic and neuronal proteins}

Katharina N. Richter ${ }^{1,2}$, Hanna Wildhagen ${ }^{1}$, Martin S. Helm ${ }^{1,3}$, Jan-Eike Ußling ${ }^{1}$, Thomas Schikorski ${ }^{4} \&$ Silvio O. Rizzoli ${ }^{1}$

${ }^{1}$ University Medical Center Göttingen, Institute for Neuro- and Sensory Physiology, Humboldtallee 23, Göttingen, 37073, Germany

${ }^{2}$ Cluster of Excellence Nanoscale Microscopy and Molecular Physiology of the Brain, Göttingen, Germany

${ }^{3}$ International Max Planck Research School Molecular Biology, Göttingen, Germany

${ }^{4}$ Department of Neuroscience, Iniversidad Central del Caribe, Bayamon, Puerto Rico, United States of America.

Correspondence and request for materials should be addressed to K.N.R. (email: k.richter1@stud.uni-goettingen.de) or S.O.R. (email: srizzol@gwdg.de) 


\subsection{Abstract}

Protein copy numbers can be measured by biochemical methods ranging from quantitative Western Blotting to several mass spectrometry approaches. Such methods only provide average copy numbers, obtained over large cell numbers. However, copy number estimates for single cells or single organelles could be obtained by combining biochemical characterizations with an imaging approach. We performed this here for synaptic proteins, in a protocol that we termed comparative synaptosome imaging for semi-quantitative copy numbers (CosiQuant). In brief, in CosiQuant we immunostain in parallel biochemicallycharacterized synaptosomes, for which we have already determined the average protein copy numbers, and the samples of interest (such as neuronal cultures). We then derive the copy numbers in the samples of interest by comparing the immunofluorescence intensities. We measured the intensities not only in arbitrary fluorescence units, but also as numbers of antibodies per synaptosome, for a large number of targets. This implies that other groups can immediately apply CosiQuant for these targets, by simply estimating the number of antibodies per structure of interest. CosiQuant should therefore be a useful addition to the growing set of imaging techniques for synaptic neuroscience.

\subsection{Introduction}

The quantitative organization of neurons has been the subject of countless scientific studies during the past decades, with synapse physiology being one of the most studied areas of neuroscience (Südhof, 2004; Haucke et al, 2011; Rizzoli, 2014). The research focus has typically been on explaining the mechanisms of neuronal communication, and finally on understanding the causes for neurological disorders.

Such disorders generally produce slight but significant changes in the basic molecular anatomy of the neuron, meaning the spatial organization and copy numbers of proteins within the neuron. The information on protein copy numbers in compartments such as the synapse is especially important, as it enables us to identify potential bottlenecks of cellular and molecular mechanisms (Haass \& Selkoe, 2007; Südhof, 2008; Kauer \& Malenka, 2007). As an example, mapping the protein composition of the synaptic vesicle (Takamori et al, 2006) has shown that the vesicular proton pump is only present in $1-2$ copies per vesicle, and is thus one of the most likely molecules to generate a bottleneck (a rate-limiting factor) in synaptic vesicle recycling mechanisms. As a second example, endocytosis cofactors have also been shown to be limiting in the vesicle recycling pathway, while the exocytosis-related proteins are far more abundant, and are unlikely to be limiting (Denker et al, 2011; Wilhelm et al, 2014). In addition, knowledge on absolute copy numbers is also desirable from a technical point of view, as it renders comparisons between independent studies far more precise. 
Numerous methods have been therefore developed to determine protein copy numbers, mainly by biochemical means. These techniques comprise Quantitative Western Blotting (Jahn et al, 1984) and mass spectrometry (MS) approaches like iBAQ (intensity-based absolute quantification (Schwanhäusser et al, 2011)) and AQUA (absolute quantification approach (Gerber et al, 2003)). Quantitative Western Blotting relies on the comparison of SDS-PAGE band intensities between a sample of interest and purified recombinant variants of the protein of interest, run on the gels in known amounts (Wilhelm et al, 2014). In many mass spectrometry approaches the estimation of the abundance of particular proteins is accomplished by comparing the spectra of the analyzed proteins and/or peptides to a standard. In AQUA this standard comprises an isotopically labeled peptide that reproduces a region of the protein of interest (Gerber et al, 2003), and which is spiked into the analyzed sample. iBAQ is a label-free method, in which one spikes accurately quantified proteins in the samples of interest, followed by in-solution digestion and mass spectrometry analysis. The intensities of the different peptides identified are summed to generate the overall protein intensities (for each protein of interest), and these are correlated to the known spiked amounts. This enables the use of a relatively simple linear regression to identify the amounts of the native proteins of interest (Schwanhäusser et al, 2011; Lu et al, 2007; Malmström et al, 2009).

In all of these approaches the samples are usually cell culture lysates or brain homogenates, which precludes the analysis of variations between single cells or cell regions. To account for this, methods combining mass spectrometry and an imaging technique have been developed, like MALDI-TOF-TOF (matrix-assisted laser desorption ionization coupled to a tandem time-of-flight analysis) imaging, which makes it possible to map the intensities of MS spectra to the corresponding position in a sample (Caprioli et al, 1997). However, accurate protein quantification is difficult to achieve, and the spatial resolution is still too low (usually about $20-50 \mu \mathrm{m}$ ) to obtain sub-cellular information. Alternatively, approaches for protein quantification based purely on imaging techniques could, in principle, provide the resolution needed for comparisons of protein copy numbers between cells or sub-cellular regions. For example, one can express GFP-tagged proteins of interest in the sample, followed by immunostaining for the proteins of interest (Sugiyama et al, 2005). One can then obtain estimates for the copy numbers of native (wild-type) proteins present in each cellular location by comparing the immunostaining signals to an estimation of the GFP copy numbers. However, these techniques are often difficult, as both the GFP signals and the immunostainings need to be carefully calibrated, and can only be applied to samples where the expression of GFP-tagged proteins is efficient.

Here we take advantage of synaptosomes that we have previously characterized by biochemical methods (Wilhelm et al, 2014) to establish a relatively easy imaging-based method for the estimation of protein copy numbers in neurons: comparative synaptosome imaging for semi-quantitative copy numbers (CosiQuant). Our approach relies on immunostaining the samples of interest and these synaptosomes in parallel. We then compare the resulting intensities in a semi-automated fashion. Protein copy numbers in the sample of interest can then be inferred from the known values in the synaptosome 
preparations, for which we derived estimates for more than 1000 proteins (Wilhelm et al, 2014). We tested this technique on cultured hippocampal neurons, and found that it works well for the estimation of neuronal proteins in this system.

Furthermore, we generalized this method, by removing the need for other laboratories to compare directly fluorescence intensities in our biochemically-characterized synaptosomes and in their preparations of interest. We expressed the fluorescence intensities we measured in synaptosomes in the form of "average numbers of antibodies per synaptosome". This implies that other laboratories can estimate the numbers of antibodies in their structures of interest, and can then compare them with the numbers we provide, which would enable the estimation of protein copy numbers. We were able to validate this approach by turning to older datasets from the laboratory, not collected for the purpose of this work, which thus served as an independent control.

\subsection{Results}

For an initial proof-of-principle, we tested the comparative imaging approach on a very well established system, the primary hippocampal neuron culture. The protocol works as follows (Fig. 1): frozen synaptosome preparations, which have been biochemically characterized in a previous study (Wilhelm et al, 2014), are thawed and are immobilized on coverslips. The synaptosomes and cultured hippocampal neurons are then immunostained in parallel for two synapse markers (synaptophysin for synaptic vesicles, and bassoon for the active zone) and for the protein of interest (POI). The fluorescent signals are acquired in the three separate channels, and the intensities of these signals are measured. The data are further processed, and afterwards signal intensities of the POI's derived from both preparations are compared. Based on the knowledge about protein copy numbers in the synaptosome preparations, copy numbers of the POI in hippocampal neuron synapses can be interpolated. We tested the comparative imaging approach for 10 synaptic markers: the synaptic vesicle marker synaptophysin, the active zone marker bassoon, as indicated above, followed by four additional synaptic vesicle proteins (the fusion SNARE VAMP2, the glutamate transporters vGlut1/2, the calcium sensor synaptotagmin 1 , and the synaptophysin-related protein synaptogyrin), two plasma membrane SNAREs (SNAP25 and syntaxin 1), the soluble vesiclebinding protein synapsin $1 / 2$, and the endosomal protein syntaxin 12/13 (see Rizzoli, 2014 for details on the different proteins). We have previously derived copy numbers for all of these proteins in the synaptosome preparations (Wilhelm et al, 2014). At the same time, thoroughly characterized antibodies are available for immunostainings. 


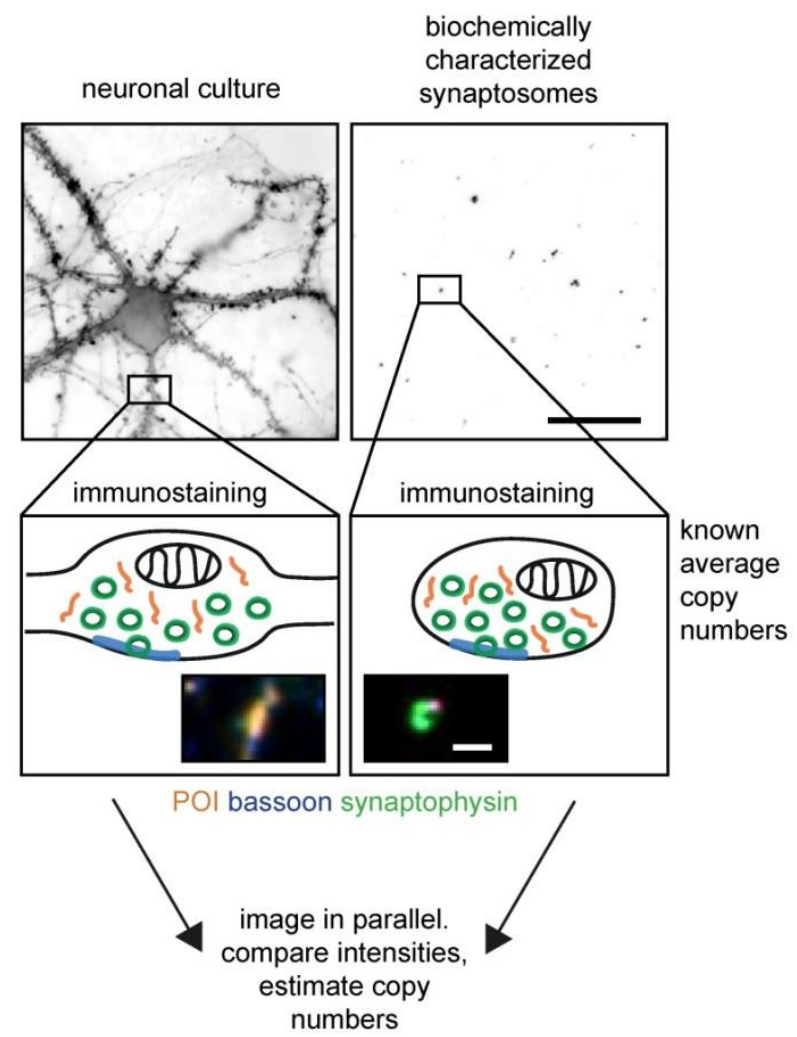

Figure 1 Principle of the comparative imaging approach (CosiQuant).

Biochemically characterized synaptosome preparations are immobilized on coverslips. Cultured hippocampal neurons are immunostained in parallel with the synaptosomes for the synaptic vesicle marker synaptophysin (green), the active zone marker bassoon (blue) and the protein of interest (orange). The insets show example images of the fluorescently labeled structures (synapses/synaptosomes). Fluorescent signals for all labeled proteins are acquired, and intensities are compared between the synaptosome and neuron samples. Finally, protein copy numbers in cultured neuronal synapses can be estimated by comparing the intensities. Scale bar in overview images $=25 \mu \mathrm{m}$; scale bar in insets $=2 \mu \mathrm{m}$.

Example images for the immunostainings are shown in Fig. 2a, with synapsin $1 / 2$ as the POI. The images, comprised of the three channels for synaptophysin, bassoon and the POI, were analyzed by a custom-written Matlab routine, as described in Methods. In a first step, potential synapses in each image were detected based on the local intensity maxima in the synaptophysin (synapse marker) signal (Fig. 2b). Application of an initial intensity threshold ensures that a huge fraction of the image noise is not taken into account. At the same time, the co-presence of the bassoon signal ensures that the detected objects are indeed synapses, and not only synaptophysin protein assemblies or vesicles transported along the neurites. For each synapse candidate the size, intensity and position are determined using a Gaussian fit (Fig. 2c). The $R^{2}$ value for each fit is calculated, and is used in a filtering step later on. Subsequently, the intensities of all three channels for all detected synapses are plotted (Fig. 2d). This data set includes many synapses that are not fitted correctly, or might not represent real/single synapses. Thus, a final filtering step has been included in the data analysis, which excludes synapse candidates with a fit worse than a set $R^{2}$ threshold. This results in a data set containing information about the size, position and intensities of synapses, carefully pruned to represent real synapses. 

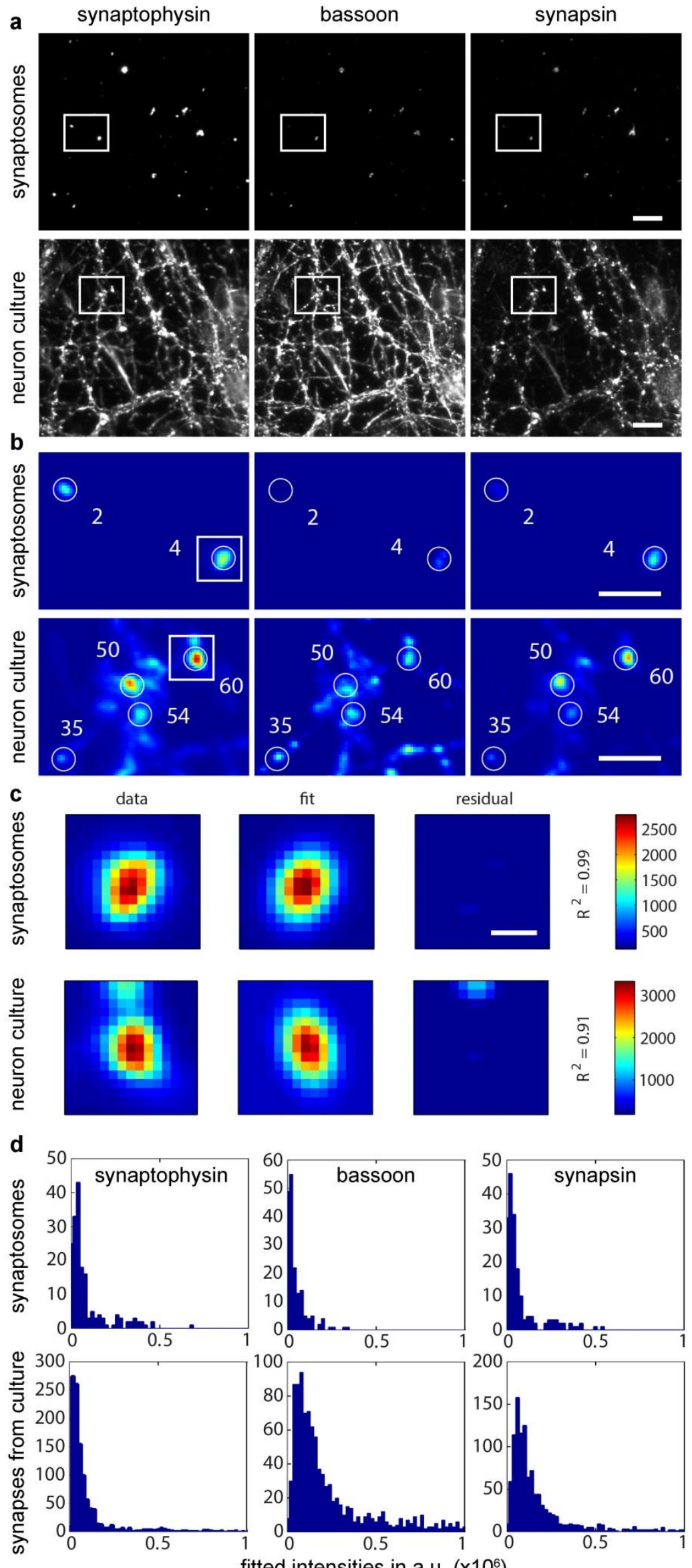

Figure $\mathbf{2}$ Image analysis for synapsin as protein of interest. 
a) Example images of synaptosomes and cultured hippocampal neurons immunostained for synaptophysin, bassoon and synapsin. Scale bar $=10 \mu \mathrm{m}$

b) Automatic synapse selection by a custom-written Matlab routine in the areas indicated in the images in a. Synapse detection is based on the signal intensities of the synaptophysin staining (first channel). The selected synapses are numbered so fitted parameters (e.g. intensity) can later be assigned to the respective signals. Scale bar $=5 \mu \mathrm{m}$

c) Example of the Gaussian fits for the synapses indicated in panel b. The first image shows the original intensity data (scale in arbitrary units on the far right), the second image shows the model that is fitted to the data, and the third image indicates the residuals, i.e. the deviations of the data from the 2D Gaussian distribution. The R2 values of each fit are indicated on the right. Images are scaled in pixel. Scale bar $=800 \mathrm{~nm}$ ( 5 pixels)

d) Intensity histograms of all fitted synapses for the three channels (prior to final filtering).

For further analysis, we collected the measured intensities for all POIs, derived from all images. Mean intensities were calculated and were compared between stainings of synaptosomes and cultured neurons (Fig. 3a). We first investigated synaptophysin, which is the most specific marker of synaptic vesicles (Takamori et al, 2006; Jahn et al, 1984; Rizzoli et al, 2006), being found almost exclusively in vesicles, unlike other vesicle proteins that are often also found on the plasma membrane (for example synaptotagmin 1 or VAMP2(Wienisch \& Klingauf, 2006; Truckenbrodt et al, 2018b)). The number of synaptic vesicles per synaptosome averages $\sim 380$ (Wilhelm et al, 2014), measured in electron microscopy. In cultured hippocampal synapses only $250 \pm 26$ synaptic vesicles are present on average (mean \pm SEM). This was calculated from 30 electron microscopy 3D reconstructions, with the following individual vesicle numbers: $36,86,89,95,119,124,128,136,137,173$, $185,191,197,197,212,224,233,248,249,252,257,302,315,377,403,450,509,516,526$, 544 . This analysis indicates that the synaptosomes contain on average $35 \%$ more synaptic vesicles. A similar value was found for the mean synaptophysin signal in our intensity comparison (29\%), suggesting that these immunostaining signals are a reliable measure for the relative protein numbers in synaptosomes and hippocampal neurons. 

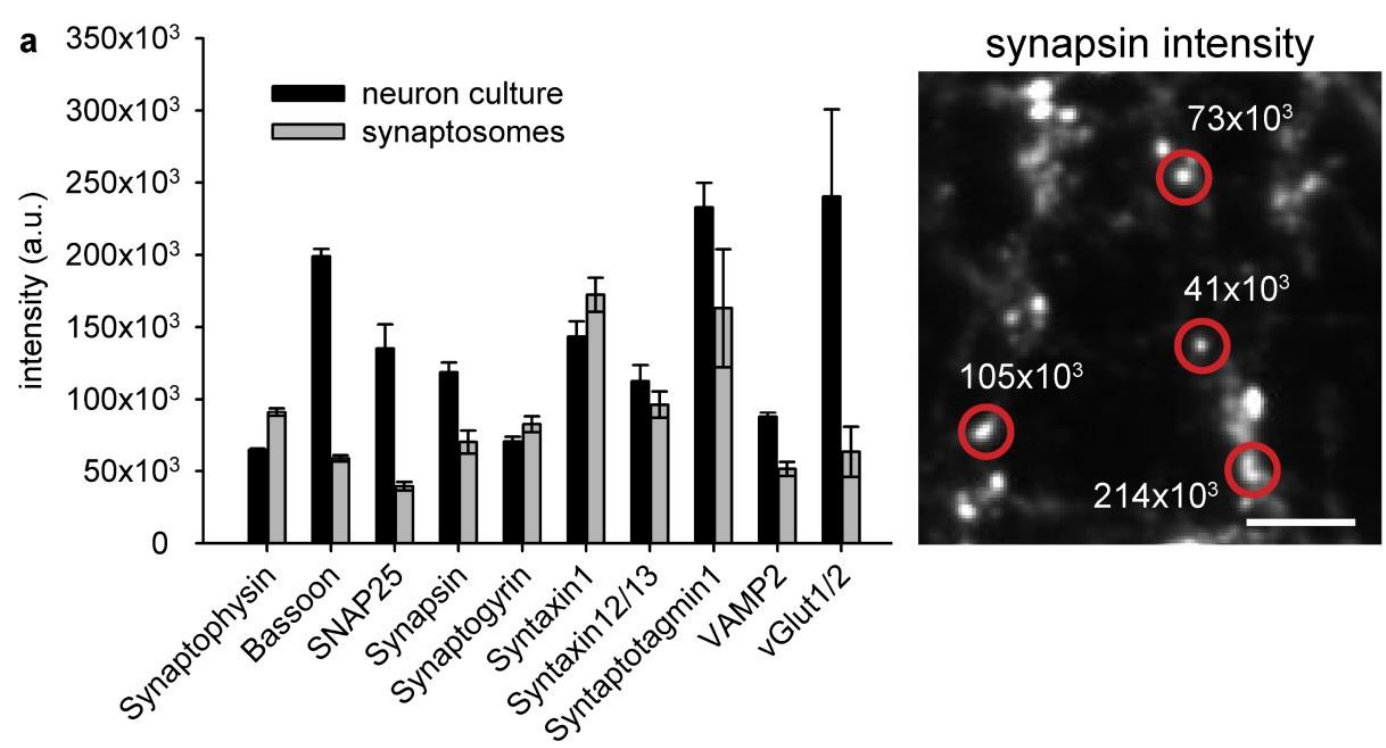

b

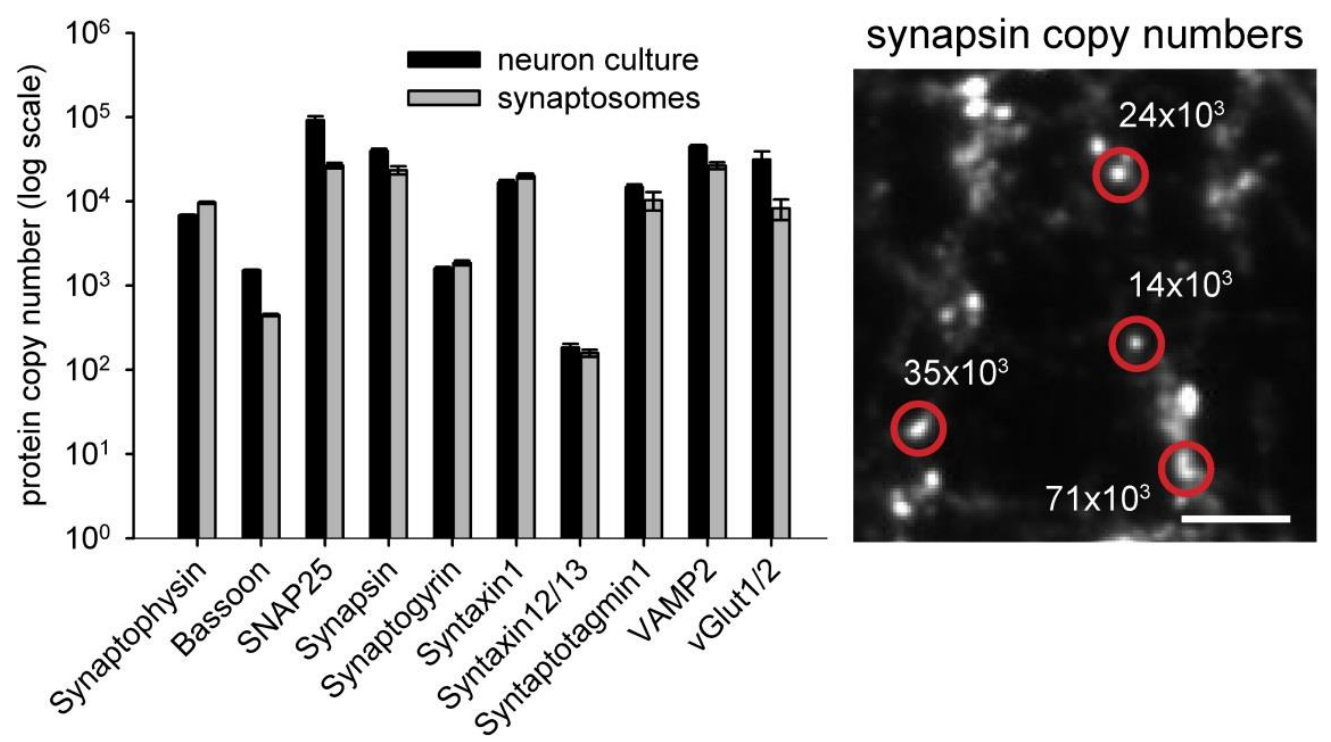

Figure 3 Comparison of intensities derived from labeled proteins in synaptosomes and neuronal synapses.

a) Absolute intensities (in arbitrary units) of the 10 labeled proteins in synaptosome preparations and cultured hippocampal neurons, expressed as mean values derived from all selected synapses (after final filtering). The right panel shows example synapses, marked with the respective intensities for the synapsin $1 / 2$ stainings (in arbitrary units). Scale bar $=5 \mu \mathrm{m}$.

b) Average synaptic protein copy numbers, obtained by dividing the synapse intensities by the average synaptosome intensities, and multiplying with the average copy numbers per synaptosome from Wilhelm et al, 2014. Example synapses with the respective synapsin $1 / 2$ copy numbers are shown in the right panel. Scale bar $=5 \mu \mathrm{m}$. In contrast to the graph in a), the graph in b) is shown in log scale. Bar graphs represent mean values with standard errors of the mean (SEM). $N=875$ synaptosomes (for synaptophysin and bassoon), 24 - 184 synaptosomes (for all other POI's), 1809 cultured synapses (for synaptophysin and bassoon), and 51 - 354 synapses (for all other POI's).

Conclusions about the copy numbers of the other investigated proteins can now be drawn in a similar fashion, and absolute copy numbers can be estimated by comparisons with protein copy numbers from (Wilhelm et al, 2014), by dividing the fluorescence intensities of the neuronal synapses by the average synaptosome intensity, followed by multiplying the result 
with the (published) average copy numbers per synaptosome (Fig. 3b). Nevertheless, this is only useful if one can use the exact same synaptosome preparations analyzed by Wilhelm and colleagues (Wilhelm et al, 2014) for the comparative staining experiments. Since this is not the case for other laboratories, we also provide here the number of single antibodies (used for the immunostainings) per synaptosome for over 100 different targets (Table 1 and 2). This will enable the comparison to immunostainings of any sample of choice performed with the same antibody, without the need for immunolabeling again the synaptosomes. This procedure only requires one to immunostain the protein of interest in the sample of choice, and to determine the number of single antibodies per structure of interest (Fig 4). The number of antibodies can then be compared between synaptosomes (Table 1 and 2) and the sample of choice, and thus protein copy numbers can be estimated.

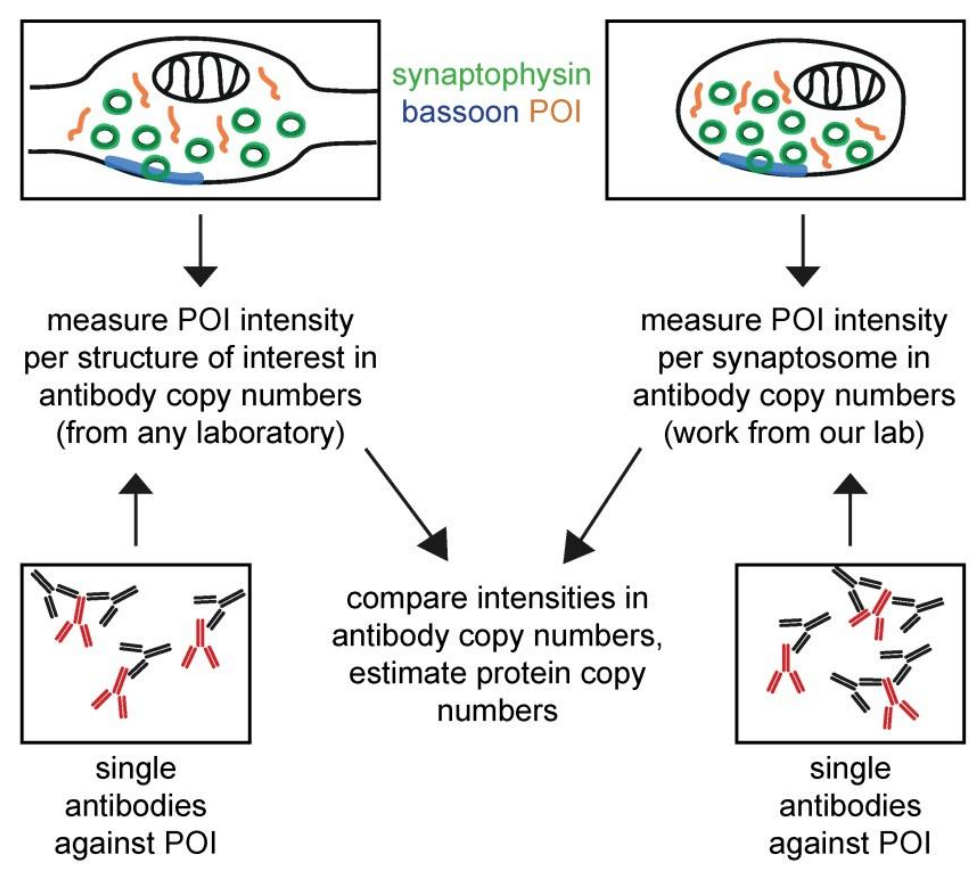

Figure 4 A comparative approach based on using the "number of antibodies per structure" as a measure for immunostaining intensities.

The intensity of the immunolabaled synaptosomes and structure of interest (here: cultured neuronal synapses) can be measured (upper panels). Fluorescent intensities from single antibodies absorbed to a glass coverslip and immunolabeled with the same secondary antibodies as used for the synaptosomes/synapses can be determined in parallel (lower panels). This makes it possible to express the intensity of labeled synaptosomes and structures of interest in terms of antibody copy numbers. This has been done by our laboratory for over 100 target proteins in synaptosomes, and can be done easily for any structure of interest by any other laboratory. Finally, intensities can be compared in terms of antibody copy numbers, and protein copy numbers can be calculated from this.

In order to validate this approach, we tested it on older data from our laboratory, which were not generated for this purpose, but were available from experiments performed previously by different investigators, using the same antibodies at different points in time, between 2012 and 2014. We analyzed super-resolution images (acquired using STED microscopy) of hippocampal neuron cultures immunostained for 4 proteins, which we had 
also investigated previously in the comparative imaging experiment showcased in Fig. 3 (synapsin, synaptogyrin, syntaxin12/13 and vGlut1/2). The intensities of single antibodies spotted on coverslips (derived from the same samples) were measured (Fig. 5a), and the number of single antibodies per neuronal synapse was calculated by dividing the total intensity in a synapse by that of the single antibodies. This provides a direct, measured number of antibodies per synapse in culture, from experiments performed between 2012 and 2014. This is the type of measurement any laboratory would be able to obtain directly, in immunostaining experiments.

a
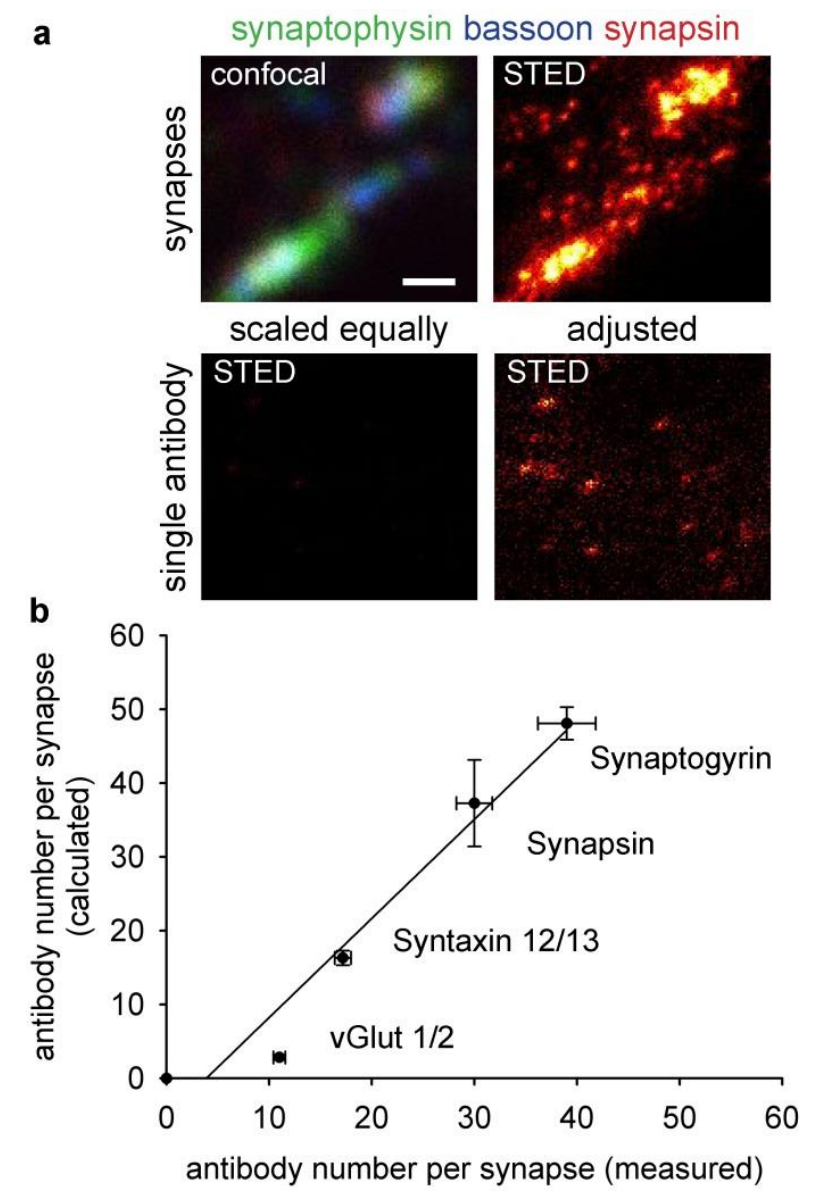

Figure 5 Numbers of single antibodies as a measure for relative immunostaining intensity.

a) Confocal and STED images of hippocampal neuron synapses and single antibodies bound to a glass coverslip. Synapses were stained for synaptophysin, bassoon and synapsin $1 / 2$ (confocal image). Super-resolution images of the signal derived from the anti-synapsin antibodies were taken in synapses (top right) or in a single-antibody configuration (bottom panels; left: scaled identically to synapses; right: autoscaled to visualize the signal of single antibodies). Scale bar $=500 \mathrm{~nm}$

b) 2-dimensional scatter plot comparing numbers of antibodies per synapse derived from the measurement described in a), along the $Y$ axis, or derived by calculation from synapse/synaptosome ratio (taken from results in Fig. 3 ), along the $X$ axis. The spots represent mean values, and error bars represent the standard error of the mean (SEM). $N=632,660,1034$ and 1009 synapses for synapsin, synaptogyrin, syntaxin $12 / 13$ and vGlut $1 / 2$ respectively, from two independent experiments, for the measured data. The estimated data are extracted from Fig. 3, and therefore have the same $\mathrm{N}$ values. The black line represents a linear regression with a slope of 1.34 and $R^{2}$ of 0.955 .

At the same time, we estimated the number of antibodies per synapse from our parallel immunostaining experiments, all performed in 2018. We simply multiplied the number of 
antibodies per synaptosome (from Table 1 and 2) with the ratio between the synaptosome and the synapse intensities, obtained from the parallel experiments from Fig. 3. This estimate should be relatively precise. We then compared the overall results in a 2dimensional scatter plot (Fig. 5b). The high correlation between the two methods indicates that the number of single antibodies per synapse is a reliable substitute for the relative intensity of an immunostaining. Therefore, numbers of antibodies per synaptosome provided by us in Table 1 and 2 can be used for the comparative imaging approach. This makes CosiQuant an easily applicable method for the estimation of protein copy numbers, with sub-cellular resolution.

Table 1 Numbers of primary antibodies per synaptosome for over 100 targets Average numbers of antibodies per synaptosome and respective information about the conditions of immunostainings from which the numbers have been derived. The values are mean values obtained either from typically several hundreds of analyzed synapses or several experiments, which were done by multiple investigators. Column $C$ shows either the standard error of the mean (SEM; in case of averaged synapses) or the range of values (ROV; in case of averaged experiments). Dil. - dilution.

\begin{tabular}{|c|c|c|c|c|c|c|c|c|}
\hline target protein & $\begin{array}{l}\text { average } \\
\text { \# of ab's }\end{array}$ & $\begin{array}{l}\text { SEM/ } \\
\text { ROV }\end{array}$ & $\begin{array}{c}\text { average \# } \\
\text { of protein } \\
\text { copies* }\end{array}$ & SEM & company & cat number & species & dil. \\
\hline Actin & 23.615 & $\begin{array}{l}1.817 \\
\text { (SEM) }\end{array}$ & 22074.6 & 1909.62 & $\begin{array}{c}\text { Novus } \\
\text { Biologicals }\end{array}$ & NB600-535 & $\begin{array}{c}\text { mouse } \\
\text { monoclonal }\end{array}$ & $1: 500$ \\
\hline ADAM22 & 20.238 & $\begin{array}{l}0.864 \\
\text { (SEM) }\end{array}$ & $* * *$ & & $\begin{array}{c}\text { Novus } \\
\text { Biologicals }\end{array}$ & $\begin{array}{l}\text { NBP2- } \\
22425\end{array}$ & $\begin{array}{c}\text { mouse } \\
\text { monoclonal }\end{array}$ & $1: 500$ \\
\hline AKT (PKB) & 10.326 & $\begin{array}{l}0.239 \\
\text { (SEM) }\end{array}$ & $* * *$ & & $\begin{array}{c}\text { Cell } \\
\text { Signaling }\end{array}$ & 4691 & $\begin{array}{c}\text { rabbit } \\
\text { monoclonal }\end{array}$ & $1: 400$ \\
\hline Alpha-SNAP & 16.216 & $\begin{array}{l}1.475 \\
\text { (SEM) }\end{array}$ & 1150.7 & 46.62 & $\begin{array}{l}\text { Synaptic } \\
\text { Systems }\end{array}$ & 112111 & $\begin{array}{c}\text { mouse } \\
\text { monoclonal }\end{array}$ & $1: 200$ \\
\hline Amphiphysin & 19.001 & $\begin{array}{l}1.973 \\
\text { (SEM) }\end{array}$ & 1194.2 & 60.04 & $\begin{array}{l}\text { Synaptic } \\
\text { Systems }\end{array}$ & 120002 & $\begin{array}{c}\text { rabbit } \\
\text { polyclonal }\end{array}$ & $1: 500$ \\
\hline AP180 & 15.174 & $\begin{array}{l}1.139 \\
\text { (SEM) } \\
\end{array}$ & 3736.4 & 207.63 & $\begin{array}{l}\text { Synaptic } \\
\text { Systems }\end{array}$ & 155003 & $\begin{array}{c}\text { rabbit } \\
\text { polyclonal }\end{array}$ & $1: 500$ \\
\hline AP2 & 19.136 & $\begin{array}{l}1.546 \\
\text { (SEM) } \\
\end{array}$ & 2324.7 & 81.99 & Abcam & ab75995 & $\begin{array}{c}\text { rabbit } \\
\text { monoclonal }\end{array}$ & $1: 500$ \\
\hline APP & 14.186 & $\begin{array}{l}2.776 \\
\text { (SEM) }\end{array}$ & 6283.6 & 584.51 & Millipore & MAB348 & $\begin{array}{c}\text { mouse } \\
\text { monoclonal }\end{array}$ & $1: 100$ \\
\hline Arc & 18.419 & $\begin{array}{l}0.545 \\
\text { (SEM) }\end{array}$ & $* * *$ & & $\begin{array}{l}\text { Synaptic } \\
\text { Systems }\end{array}$ & 156003 & $\begin{array}{c}\text { rabbit } \\
\text { polyclonal }\end{array}$ & $1: 1000$ \\
\hline BACE1 & 17.660 & $\begin{array}{l}0.886 \\
\text { (SEM) }\end{array}$ & 115.84 & 2.75 & Santa Cruz & $M-83$ & $\begin{array}{c}\text { rabbit } \\
\text { polyclonal }\end{array}$ & $1: 100$ \\
\hline Bassoon & 60.331 & $\begin{array}{l}6.221 \\
\text { (ROV) }\end{array}$ & 446.14 & 37.71 & Enzo & $\begin{array}{l}\text { ADI-VAM- } \\
\text { PSO03-F }\end{array}$ & $\begin{array}{c}\text { mouse } \\
\text { monoclonal }\end{array}$ & $1: 100$ \\
\hline Bassoon & 27.538 & $\begin{array}{l}2.626 \\
\text { (ROV) }\end{array}$ & 446.14 & 37.71 & $\begin{array}{l}\text { Synaptic } \\
\text { Systems }\end{array}$ & 141002 & $\begin{array}{c}\text { rabbit } \\
\text { polyclonal }\end{array}$ & $1: 500$ \\
\hline Calbindin & 33.403 & $\begin{array}{l}0.917 \\
\text { (ROV) }\end{array}$ & 296.88 & 13.22 & Swant & CB38a & $\begin{array}{c}\text { rabbit } \\
\text { polyclonal }\end{array}$ & $1: 500$ \\
\hline Calcineurin & 11.718 & $\begin{array}{l}0.188 \\
\text { (SEM) }\end{array}$ & $\begin{array}{c}138.67 \\
\text { (isoform B) }\end{array}$ & 91.82 & $\begin{array}{l}\text { Synaptic } \\
\text { Systems }\end{array}$ & 387002 & $\begin{array}{c}\text { rabbit } \\
\text { polyclonal }\end{array}$ & $1: 1000$ \\
\hline Calmodulin & 27.019 & $\begin{array}{l}4.493 \\
\text { (SEM) }\end{array}$ & 8659.9 & 445.47 & $\begin{array}{c}\text { Novus } \\
\text { Biologicals }\end{array}$ & $\begin{array}{c}\text { NB110- } \\
55649\end{array}$ & $\begin{array}{c}\text { rabbit } \\
\text { monoclonal }\end{array}$ & $1: 500$ \\
\hline Calmodulin & 13.510 & $\begin{array}{c}1.0193 \\
\text { (ROV) }\end{array}$ & 8659.9 & 445.47 & Abcam & ab45689 & $\begin{array}{c}\text { rabbit } \\
\text { monoclonal }\end{array}$ & $1: 100$ \\
\hline Calretinin & 16.688 & 2.724 & 369.24 & 2.47 & Swant & CR7699/4 & rabbit & $1: 500$ \\
\hline
\end{tabular}




\begin{tabular}{|c|c|c|c|c|c|c|c|c|}
\hline & & (ROV) & & & & & polyclonal & \\
\hline CAPS1 & 46.108 & $\begin{array}{c}12.0256 \\
\text { (ROV) }\end{array}$ & 196.42 & 28.58 & Abcam & ab32014 & $\begin{array}{c}\text { mouse } \\
\text { monoclonal }\end{array}$ & $1: 1000$ \\
\hline CAPS1 & 23.302 & $\begin{array}{l}2.013 \\
\text { (SEM) }\end{array}$ & 196.42 & 28.58 & Abcam & ab69797 & $\begin{array}{c}\text { rabbit } \\
\text { polyclonal }\end{array}$ & 1:500 \\
\hline $\begin{array}{l}\text { CB1 (Anti- } \\
\text { Cannabinoid } \\
\text { Receptor) }\end{array}$ & 12.842 & $\begin{array}{l}0.745 \\
\text { (ROV) }\end{array}$ & $* * *$ & & Abcam & ab23703 & $\begin{array}{c}\text { rabbit } \\
\text { polyclonal }\end{array}$ & $1: 50$ \\
\hline CDC42 & 13.380 & $\begin{array}{l}0.479 \\
\text { (SEM) }\end{array}$ & 800.4 & 268.073 & $\begin{array}{l}\text { Thermo } \\
\text { Scientific }\end{array}$ & PA1-092 & $\begin{array}{c}\text { rabbit } \\
\text { polyclonal }\end{array}$ & 1:100 \\
\hline $\begin{array}{c}\text { Chromogranin } \\
\text { A } \\
\end{array}$ & 8.852 & $\begin{array}{l}0.276 \\
\text { (ROV) }\end{array}$ & $\begin{array}{c}\text { Presumabl } \\
\text { y similar to } \\
\text { Chromogr } \\
\text { aninB }\end{array}$ & & $\begin{array}{l}\text { Synaptic } \\
\text { Systems }\end{array}$ & 259003 & $\begin{array}{c}\text { rabbit } \\
\text { polyclonal }\end{array}$ & $1: 500$ \\
\hline $\begin{array}{c}\text { Chromogranin } \\
\text { B } \\
\end{array}$ & 13.082 & $\begin{array}{l}5.006 \\
\text { (ROV) } \\
\end{array}$ & 15.67 & 4.19 & $\begin{array}{l}\text { Synaptic } \\
\text { Systems }\end{array}$ & 259103 & $\begin{array}{c}\text { rabbit } \\
\text { polyclonal }\end{array}$ & 1:500 \\
\hline $\begin{array}{c}\text { Chromogranin } \\
\text { C }\end{array}$ & 11.123 & $\begin{array}{c}0.8085 \\
(\mathrm{ROV})\end{array}$ & $\begin{array}{l}\text { Presumabl } \\
\text { y similar to } \\
\text { Chromogr } \\
\text { aninB }\end{array}$ & & Abcam & ab12241 & $\begin{array}{c}\text { rabbit } \\
\text { polyclonal }\end{array}$ & $1: 250$ \\
\hline $\begin{array}{c}\text { Clathrin Heavy } \\
\text { Chain }\end{array}$ & 37.678 & $\begin{array}{c}5.6 \\
\text { (SEM) }\end{array}$ & 3472.47 & 174.65 & $\begin{array}{c}\text { BD } \\
\text { Bioscience } \\
\text { s }\end{array}$ & 610499 & $\begin{array}{c}\text { mouse } \\
\text { monoclonal }\end{array}$ & $1: 500$ \\
\hline $\begin{array}{c}\text { Clathrin Light } \\
\text { Chain } \\
\end{array}$ & 13.465 & $\begin{array}{l}1.154 \\
\text { (ROV) }\end{array}$ & 4554.06 & 296.74 & $\begin{array}{l}\text { Synaptic } \\
\text { Systems }\end{array}$ & 113011 & $\begin{array}{c}\text { mouse } \\
\text { monoclonal }\end{array}$ & $1: 500$ \\
\hline Complexin1/2 & 15.858 & 2 (SEM) & 2488.2 & 149.49 & $\begin{array}{l}\text { Synaptic } \\
\text { Systems }\end{array}$ & 122002 & $\begin{array}{c}\text { rabbit } \\
\text { polyclonal }\end{array}$ & $1: 500$ \\
\hline CSP & 16.542 & $\begin{array}{l}3.041 \\
\text { (SEM) }\end{array}$ & 941.18 & 48.86 & $\begin{array}{l}\text { Synaptic } \\
\text { Systems }\end{array}$ & 154003 & $\begin{array}{c}\text { rabbit } \\
\text { polyclonal }\end{array}$ & $1: 500$ \\
\hline DLGAP1 & 31.073 & $\begin{array}{l}4.709 \\
\text { (ROV) }\end{array}$ & 52.961 & 27.765 & $\begin{array}{c}\text { Novus } \\
\text { Biologicals }\end{array}$ & $\begin{array}{l}\text { NBP1- } \\
76911 \\
\end{array}$ & $\begin{array}{c}\text { rabbit } \\
\text { polyclonal }\end{array}$ & $1: 50$ \\
\hline Doc $2 A / B$ & 26.170 & $\begin{array}{l}5.973 \\
\text { (ROV) } \\
\end{array}$ & 3696.5 & 164.19 & $\begin{array}{l}\text { Synaptic } \\
\text { Systems }\end{array}$ & 174203 & $\begin{array}{c}\text { rabbit } \\
\text { polyclonal }\end{array}$ & 1:500 \\
\hline $\begin{array}{c}\text { Dopamine } \\
\text { ReceptorD1 }\end{array}$ & 16.985 & $\begin{array}{c}1.9254 \\
\text { (ROV) }\end{array}$ & $* * *$ & & Abcam & ab40653 & $\begin{array}{c}\text { rabbit } \\
\text { polyclonal }\end{array}$ & $1: 1000$ \\
\hline $\begin{array}{c}\text { Dopamine } \\
\text { ReceptorD2 }\end{array}$ & 20.186 & $\begin{array}{l}0.418 \\
\text { (SEM) } \\
\end{array}$ & $* * *$ & & Merck & AB5084P & $\begin{array}{c}\text { rabbit } \\
\text { polyclonal }\end{array}$ & $1: 500$ \\
\hline Drebrin1 & 22.188 & $\begin{array}{l}0.532 \\
\text { (SEM) }\end{array}$ & 342.075 & 85.238 & $\begin{array}{c}\text { Novus } \\
\text { Biologicals }\end{array}$ & $\begin{array}{c}\text { NB100- } \\
1951 \\
\end{array}$ & $\begin{array}{c}\text { mouse } \\
\text { monoclonal }\end{array}$ & 1:100 \\
\hline Dynamin $1 / 2 / 3$ & 17.379 & $\begin{array}{l}1.382 \\
\text { (SEM) }\end{array}$ & 2326.4 & 83.87 & $\begin{array}{l}\text { Synaptic } \\
\text { Systems }\end{array}$ & 115002 & $\begin{array}{c}\text { rabbit } \\
\text { polyclonal }\end{array}$ & $1: 500$ \\
\hline Endophilinl & 12.681 & $\begin{array}{l}1.999 \\
\text { (SEM) }\end{array}$ & $\begin{array}{l}2524.4 \text { (all } \\
\text { isoforms) }\end{array}$ & 67.27 & $\begin{array}{l}\text { Synaptic } \\
\text { Systems }\end{array}$ & 159002 & $\begin{array}{c}\text { rabbit } \\
\text { polyclonal }\end{array}$ & $1: 500$ \\
\hline Epsin1 & 20.954 & $\begin{array}{l}1.306 \\
\text { (SEM) } \\
\end{array}$ & 92.88 & 4.3 & $\begin{array}{c}\text { Novus } \\
\text { Biologicals }\end{array}$ & EPR3023 & $\begin{array}{c}\text { rabbit } \\
\text { polyclonal }\end{array}$ & 1:100 \\
\hline ERp72 (PDIA4) & 17.546 & $\begin{array}{l}0.585 \\
\text { (SEM) }\end{array}$ & $* * *$ & & $\begin{array}{c}\text { Cell } \\
\text { Signaling }\end{array}$ & 5033 & $\begin{array}{c}\text { rabbit } \\
\text { monoclonal }\end{array}$ & $1: 100$ \\
\hline $\begin{array}{c}\text { GluK1 (Kainate } \\
\text { Receptor) }\end{array}$ & 15.725 & $\begin{array}{l}2.207 \\
\text { (ROV) }\end{array}$ & $* * *$ & & Alomone & AGC-008 & $\begin{array}{c}\text { rabbit } \\
\text { polyclonal }\end{array}$ & 1:100 \\
\hline $\begin{array}{c}\text { GluN1 (Kainate } \\
\text { Receptor) }\end{array}$ & 15.382 & $\begin{array}{l}2.161 \\
\text { (SEM) }\end{array}$ & $* * *$ & & $\begin{array}{l}\text { Synaptic } \\
\text { Systems }\end{array}$ & 114011 & $\begin{array}{c}\text { mouse } \\
\text { monoclonal }\end{array}$ & $1: 1000$ \\
\hline $\begin{array}{c}\text { GluN2A } \\
\text { (Glutamate } \\
\text { Receptor) }\end{array}$ & 23.966 & $\begin{array}{l}1.091 \\
\text { (SEM) }\end{array}$ & $* * *$ & & NeuroMab & $75-288$ & $\begin{array}{c}\text { mouse } \\
\text { monoclonal }\end{array}$ & 1:100 \\
\hline $\begin{array}{c}\text { GluN2B } \\
\text { (Glutamate }\end{array}$ & 8.549 & $\begin{array}{c}1.06 \\
\text { (SEM) }\end{array}$ & 83.6224 & 20.6121 & NeuroMab & $75-101$ & $\begin{array}{c}\text { mouse } \\
\text { monoclonal }\end{array}$ & $1: 100$ \\
\hline
\end{tabular}




\begin{tabular}{|c|c|c|c|c|c|c|c|c|}
\hline Receptor) & & & & & & & & \\
\hline $\begin{array}{c}\text { GluR3 } \\
\text { (Glutamate } \\
\text { Receptor) }\end{array}$ & 20.061 & $\begin{array}{l}0.998 \\
\text { (SEM) } \\
\end{array}$ & $* * *$ & & $\begin{array}{c}\text { Invitrogen } \\
\text { /Life } \\
\text { Technologi } \\
\text { es } \\
\end{array}$ & $32-0400$ & $\begin{array}{c}\text { mouse } \\
\text { monoclonal }\end{array}$ & $1: 100$ \\
\hline Homer1 & 9.363 & $\begin{array}{l}1.947 \\
\text { (SEM) }\end{array}$ & 712.817 & 83.691 & $\begin{array}{l}\text { Synaptic } \\
\text { Systems }\end{array}$ & 160011 & $\begin{array}{c}\text { mouse } \\
\text { monoclonal }\end{array}$ & $1: 500$ \\
\hline Homer3 & 12.718 & $\begin{array}{l}0.691 \\
\text { (ROV) }\end{array}$ & 207.483 & 66.367 & $\begin{array}{l}\text { Synaptic } \\
\text { Systems }\end{array}$ & 160303 & $\begin{array}{c}\text { rabbit } \\
\text { polyclonal }\end{array}$ & $1: 250$ \\
\hline $\mathrm{Hsc} 70$ & 24.154 & $\begin{array}{c}3.72 \\
\text { (SEM) }\end{array}$ & 8210.1 & 404.5 & Santa Cruz & sc7298 & $\begin{array}{c}\text { mouse } \\
\text { monoclonal }\end{array}$ & $1: 500$ \\
\hline IGF-1R & 10.494 & $\begin{array}{l}0.935 \\
\text { (ROV) }\end{array}$ & $* * *$ & & $\begin{array}{c}\text { Cell } \\
\text { Signaling }\end{array}$ & 3027 & $\begin{array}{c}\text { rabbit } \\
\text { polyclonal }\end{array}$ & $1: 300$ \\
\hline Intersectin1 & 26.077 & $\begin{array}{l}2.955 \\
\text { (SEM) }\end{array}$ & 3096.5 & 277.62 & $\begin{array}{l}\text { Haucke } \\
\text { (Berlin) }\end{array}$ & & $\begin{array}{c}\text { rabbit } \\
\text { polyclonal }\end{array}$ & $1: 500$ \\
\hline Kir2.1 & 8.312 & $\begin{array}{c}0.3 \\
\text { (ROV) }\end{array}$ & $* * *$ & & $\begin{array}{c}\text { Novus } \\
\text { Biologicals }\end{array}$ & $\begin{array}{l}\text { NBP1- } \\
95482 \\
\end{array}$ & $\begin{array}{c}\text { rabbit } \\
\text { monoclonal }\end{array}$ & $1: 100$ \\
\hline Kv1.1 & 13.047 & $\begin{array}{l}4.988 \\
\text { (ROV) }\end{array}$ & 201.2956 & 95.671 & $\begin{array}{l}\text { Thermo } \\
\text { Scientific }\end{array}$ & PA5-19593 & $\begin{array}{c}\text { rabbit } \\
\text { polyclonal }\end{array}$ & $1: 100$ \\
\hline Kv2.1 & 10.647 & $\begin{array}{l}1.674 \\
\text { (ROV) }\end{array}$ & $* * *$ & & $\begin{array}{l}\text { Synaptic } \\
\text { Systems }\end{array}$ & 231002 & $\begin{array}{c}\text { rabbit } \\
\text { polyclonal }\end{array}$ & $1: 500$ \\
\hline Munc13a & 19.037 & $\begin{array}{l}1.047 \\
\text { (SEM) } \\
\end{array}$ & 1551.3 & 53.18 & $\begin{array}{l}\text { Synaptic } \\
\text { Systems }\end{array}$ & 126102 & $\begin{array}{c}\text { rabbit } \\
\text { polyclonal }\end{array}$ & $1: 500$ \\
\hline Munc18a & 21.108 & $\begin{array}{c}1.53 \\
\text { (SEM) }\end{array}$ & 4253.4 & 207.07 & $\begin{array}{l}\text { Synaptic } \\
\text { Systems }\end{array}$ & 116002 & $\begin{array}{c}\text { rabbit } \\
\text { polyclonal }\end{array}$ & $1: 500$ \\
\hline Myosin5a & 9.245 & $\begin{array}{l}1.271 \\
\text { (SEM) }\end{array}$ & 157.24 & 20 & $\begin{array}{l}\text { Sigma- } \\
\text { Aldrich }\end{array}$ & M5062 & $\begin{array}{c}\text { rabbit } \\
\text { polyclonal }\end{array}$ & $1: 200$ \\
\hline NavBeta1 & 18.611 & $\begin{array}{l}1.972 \\
\text { (SEM) }\end{array}$ & 830.8294 & $\begin{array}{c}248.918 \\
8 \\
\end{array}$ & Alomone & ASC-041 & $\begin{array}{c}\text { rabbit } \\
\text { polyclonal }\end{array}$ & $1: 50$ \\
\hline nAChRBeta2 & 21.164 & $\begin{array}{l}0.718 \\
\text { (SEM) } \\
\end{array}$ & $* * *$ & & Alomone & ABC-012 & $\begin{array}{c}\text { rabbit } \\
\text { polyclonal }\end{array}$ & $1: 100$ \\
\hline NaKATPase & 29.913 & $\begin{array}{l}0.961 \\
\text { (SEM) }\end{array}$ & 3771.7 & 481.7 & $\begin{array}{l}\text { Thermo } \\
\text { Scientific }\end{array}$ & MA3-915 & $\begin{array}{c}\text { mouse } \\
\text { monoclonal }\end{array}$ & $1: 1000$ \\
\hline Nav1.1 & 13.934 & $\begin{array}{l}1.261 \\
\text { (SEM) } \\
\end{array}$ & $* * *$ & & Merck & $06-811$ & $\begin{array}{c}\text { rabbit } \\
\text { polyclonal }\end{array}$ & $1: 100$ \\
\hline Nav1.3 & 10.685 & $\begin{array}{l}0.805 \\
\text { (SEM) }\end{array}$ & $* * *$ & & Alomone & ASC-004 & $\begin{array}{c}\text { rabbit } \\
\text { polyclonal }\end{array}$ & $1: 250$ \\
\hline nNOS & 7.441 & $\begin{array}{l}0.773 \\
\text { (ROV) }\end{array}$ & $* * *$ & & $\begin{array}{l}\text { Thermo } \\
\text { Scientific }\end{array}$ & PA1-033 & $\begin{array}{c}\text { rabbit } \\
\text { polyclonal }\end{array}$ & $1: 100$ \\
\hline NSF & 15.662 & $\begin{array}{l}0.432 \\
\text { (SEM) } \\
\end{array}$ & 677.45 & 213.02 & $\begin{array}{l}\text { Synaptic } \\
\text { Systems }\end{array}$ & 123002 & $\begin{array}{c}\text { rabbit } \\
\text { polyclonal }\end{array}$ & $1: 100$ \\
\hline NSF & 17.220 & $\begin{array}{l}0.976 \\
\text { (SEM) } \\
\end{array}$ & 677.45 & 213.02 & $\begin{array}{l}\text { Synaptic } \\
\text { Systems }\end{array}$ & 123002 & $\begin{array}{c}\text { rabbit } \\
\text { polyclonal }\end{array}$ & $1: 500$ \\
\hline Parvalbumin & 33.327 & $\begin{array}{l}0.435 \\
\text { (ROV) }\end{array}$ & 681.1 & 34.31 & Swant & PV25 & $\begin{array}{c}\text { rabbit } \\
\text { polyclonal }\end{array}$ & $1: 500$ \\
\hline Piccolo & 32.092 & $\begin{array}{l}0.322 \\
\text { (ROV) } \\
\end{array}$ & 100.45 & 8.4 & $\begin{array}{l}\text { Synaptic } \\
\text { Systems }\end{array}$ & 142003 & $\begin{array}{c}\text { rabbit } \\
\text { polyclonal }\end{array}$ & $1: 200$ \\
\hline PSD95 & 17.742 & $\begin{array}{l}1.163 \\
\text { (SEM) } \\
\end{array}$ & 1500.2 & 349.9 & Sigma & P246 & $\begin{array}{c}\text { mouse } \\
\text { monoclonal }\end{array}$ & $1: 200$ \\
\hline PSD95 & 19.390 & $\begin{array}{l}1.387 \\
\text { (SEM) }\end{array}$ & 1500.2 & 349.9 & $\begin{array}{c}\text { Cell } \\
\text { Signaling }\end{array}$ & 3450 & $\begin{array}{c}\text { rabbit } \\
\text { monoclonal }\end{array}$ & $1: 100$ \\
\hline Rab3a & 19.254 & $\begin{array}{l}2.613 \\
\text { (SEM) } \\
\end{array}$ & 18846.58 & 996.01 & $\begin{array}{l}\text { Synaptic } \\
\text { Systems }\end{array}$ & 107003 & $\begin{array}{c}\text { rabbit } \\
\text { polyclonal }\end{array}$ & $1: 500$ \\
\hline Rab5a & 29.144 & $\begin{array}{l}4.168 \\
\text { (SEM) } \\
\end{array}$ & 633.62 & 37.26 & $\begin{array}{c}\text { Cell } \\
\text { Signaling }\end{array}$ & $3547 \mathrm{~S}$ & $\begin{array}{c}\text { rabbit } \\
\text { polyclonal }\end{array}$ & $1: 500$ \\
\hline Rab7a & 21.976 & 2.29 & 4457.2 & 319.8 & Santa Cruz & sc81922 & rabbit & $1: 100$ \\
\hline
\end{tabular}




\begin{tabular}{|c|c|c|c|c|c|c|c|c|}
\hline & & (SEM) & & & & & polyclonal & \\
\hline Rab9 & 10.173 & $\begin{array}{l}1.552 \\
\text { (ROV) }\end{array}$ & $* * *$ & & $\begin{array}{c}\text { Cell } \\
\text { Signaling }\end{array}$ & 5118 & $\begin{array}{c}\text { rabbit } \\
\text { polyclonal }\end{array}$ & $1: 100$ \\
\hline Rapsn & 15.000 & $\begin{array}{l}1.443 \\
\text { (SEM) } \\
\end{array}$ & $* * *$ & & $\begin{array}{c}\text { Atlas } \\
\text { Antibodies }\end{array}$ & HPA039475 & $\begin{array}{c}\text { rabbit } \\
\text { polyclonal }\end{array}$ & $1: 100$ \\
\hline Rim1 & 22.028 & $\begin{array}{l}1.419 \\
\text { (ROV) }\end{array}$ & 38.63 & 4.23 & $\begin{array}{l}\text { Synaptic } \\
\text { Systems }\end{array}$ & 140003 & $\begin{array}{c}\text { rabbit } \\
\text { polyclonal }\end{array}$ & $1: 200$ \\
\hline SCAMP1 & 34.862 & $\begin{array}{l}2.961 \\
\text { (SEM) }\end{array}$ & 1459.5 & 115.53 & $\begin{array}{l}\text { Synaptic } \\
\text { Systems }\end{array}$ & 121002 & $\begin{array}{c}\text { rabbit } \\
\text { polyclonal }\end{array}$ & $1: 100$ \\
\hline $\operatorname{Sec} 22 b$ & 15.399 & $\begin{array}{c}9.38 \\
\text { (ROV) }\end{array}$ & 118.69 & 38.72 & $\begin{array}{l}\text { Synaptic } \\
\text { Systems }\end{array}$ & 186003 & $\begin{array}{c}\text { rabbit } \\
\text { polyclonal }\end{array}$ & $1: 100$ \\
\hline Septin5 & 24.894 & $\begin{array}{l}1.786 \\
\text { (SEM) } \\
\end{array}$ & 1726.2 & 64.38 & $\begin{array}{l}\text { Haucke } \\
\text { (Berlin) }\end{array}$ & & $\begin{array}{c}\text { rabbit } \\
\text { polyclonal }\end{array}$ & $1: 500$ \\
\hline Septin7 & 14.637 & $\begin{array}{l}0.168 \\
\text { (SEM) }\end{array}$ & 2320.5 & 98.66 & $\begin{array}{c}\text { Atlas } \\
\text { Antibodies }\end{array}$ & HPA029524 & $\begin{array}{c}\text { rabbit } \\
\text { polyclonal }\end{array}$ & $1: 50$ \\
\hline Shank1 & 10.116 & $\begin{array}{l}0.515 \\
\text { (SEM) } \\
\end{array}$ & 141.276 & 35.11 & $\begin{array}{l}\text { Synaptic } \\
\text { Systems }\end{array}$ & 162013 & $\begin{array}{c}\text { rabbit } \\
\text { polyclonal }\end{array}$ & $1: 500$ \\
\hline Shank2 & 15.660 & $\begin{array}{l}0.638 \\
\text { (ROV) } \\
\end{array}$ & 168.2534 & 12.6241 & $\begin{array}{l}\text { Synaptic } \\
\text { Systems }\end{array}$ & 162202 & $\begin{array}{c}\text { rabbit } \\
\text { polyclonal }\end{array}$ & $1: 500$ \\
\hline Shank3 & 22.504 & $\begin{array}{l}3.638 \\
\text { (ROV) }\end{array}$ & 181.28 & 53.24 & $\begin{array}{l}\text { Synaptic } \\
\text { Systems }\end{array}$ & 162302 & $\begin{array}{c}\text { rabbit } \\
\text { polyclonal }\end{array}$ & $1: 500$ \\
\hline SNAP23 & 12.955 & $\begin{array}{l}0.997 \\
\text { (SEM) } \\
\end{array}$ & 265.61 & 17.75 & $\begin{array}{l}\text { Synaptic } \\
\text { Systems }\end{array}$ & 111202 & $\begin{array}{c}\text { rabbit } \\
\text { polyclonal }\end{array}$ & $1: 100$ \\
\hline SNAP 25 & 10.415 & $\begin{array}{c}2.71 \\
\text { (ROV) } \\
\end{array}$ & 26686.08 & 5287.39 & $\begin{array}{l}\text { Synaptic } \\
\text { Systems }\end{array}$ & 111011 & $\begin{array}{c}\text { mouse } \\
\text { monoclonal }\end{array}$ & $1: 100$ \\
\hline SNAP29 & 19.561 & $\begin{array}{l}2.654 \\
\text { (SEM) }\end{array}$ & 77.47 & 6.47 & $\begin{array}{l}\text { Synaptic } \\
\text { Systems }\end{array}$ & 111302 & $\begin{array}{c}\text { rabbit } \\
\text { polyclonal }\end{array}$ & $1: 500$ \\
\hline SNAP29 & 7.413 & $\begin{array}{c}0.79 \\
\text { (ROV) }\end{array}$ & 77.47 & 6.47 & $\begin{array}{l}\text { Synaptic } \\
\text { Systems }\end{array}$ & 111302 & $\begin{array}{c}\text { rabbit } \\
\text { polyclonal }\end{array}$ & $1: 500$ \\
\hline SNAP47 & 9.079 & $\begin{array}{l}0.546 \\
\text { (ROV) }\end{array}$ & $\sim 200 * *$ & & $\begin{array}{l}\text { Synaptic } \\
\text { Systems }\end{array}$ & 111403 & $\begin{array}{c}\text { rabbit } \\
\text { polyclonal }\end{array}$ & $1: 200$ \\
\hline Stargazin & 5.314 & $\begin{array}{l}0.296 \\
\text { (SEM) }\end{array}$ & 143.78 & 12.33 & Alomone & ACC-012 & $\begin{array}{c}\text { rabbit } \\
\text { polyclonal }\end{array}$ & $1: 200$ \\
\hline SV2A/B & 16.201 & $\begin{array}{c}1.63 \\
\text { (SEM) }\end{array}$ & 4616.65 & 128.17 & $\begin{array}{c}\text { Jahn } \\
\text { Departme } \\
\text { nt }\end{array}$ & & $\begin{array}{c}\text { mouse } \\
\text { monoclonal }\end{array}$ & $1: 100$ \\
\hline Synapsin1/2 & 21.991 & $\begin{array}{l}3.462 \\
\text { (SEM) }\end{array}$ & 23422.77 & 1300.03 & $\begin{array}{l}\text { Synaptic } \\
\text { Systems }\end{array}$ & 106002 & $\begin{array}{c}\text { rabbit } \\
\text { polyclonal }\end{array}$ & $1: 500$ \\
\hline Synaptogyrin & 56.224 & $\begin{array}{c}2.57 \\
\text { (SEM) } \\
\end{array}$ & 1854.8 & 110.49 & $\begin{array}{l}\text { Synaptic } \\
\text { Systems }\end{array}$ & 103002 & $\begin{array}{c}\text { rabbit } \\
\text { polyclonal }\end{array}$ & $1: 100$ \\
\hline Synaptojanin & 19.497 & $\begin{array}{l}0.712 \\
\text { (SEM) } \\
\end{array}$ & 365.61 & 40.31 & $\begin{array}{l}\text { Synaptic } \\
\text { Systems }\end{array}$ & 145003 & $\begin{array}{c}\text { rabbit } \\
\text { polyclonal }\end{array}$ & $1: 100$ \\
\hline $\begin{array}{c}\text { Synaptotagmin } \\
1\end{array}$ & 15.737 & $\begin{array}{l}1.671 \\
\text { (SEM) }\end{array}$ & 10332 & 1079.2 & $\begin{array}{l}\text { Synaptic } \\
\text { Systems }\end{array}$ & 105102 & $\begin{array}{c}\text { rabbit } \\
\text { polyclonal }\end{array}$ & $1: 1000$ \\
\hline $\begin{array}{c}\text { Synaptotagmin } \\
2 \\
\end{array}$ & 15.451 & $\begin{array}{l}1.823 \\
\text { (SEM) }\end{array}$ & 297.28 & 11.37 & $\begin{array}{l}\text { Synaptic } \\
\text { Systems }\end{array}$ & 105123 & $\begin{array}{c}\text { rabbit } \\
\text { polyclonal }\end{array}$ & $1: 100$ \\
\hline $\begin{array}{c}\text { Synaptotagmin } \\
4 \\
\end{array}$ & 9.333 & $\begin{array}{l}0.795 \\
\text { (ROV) } \\
\end{array}$ & $* * *$ & & $\begin{array}{l}\text { Synaptic } \\
\text { Systems }\end{array}$ & 105143 & $\begin{array}{c}\text { rabbit } \\
\text { polyclonal }\end{array}$ & $1: 1000$ \\
\hline $\begin{array}{c}\text { Synaptotagmin } \\
5 / 9\end{array}$ & 18.341 & $\begin{array}{l}0.318 \\
\text { (SEM) }\end{array}$ & $* * *$ & & $\begin{array}{l}\text { Synaptic } \\
\text { Systems }\end{array}$ & 105053 & $\begin{array}{c}\text { rabbit } \\
\text { polyclonal }\end{array}$ & $1: 100$ \\
\hline $\begin{array}{c}\text { Synaptotagmin } \\
7 \\
\end{array}$ & 29.852 & $\begin{array}{l}1.613 \\
\text { (SEM) }\end{array}$ & 182.64 & 3.54 & $\begin{array}{l}\text { Synaptic } \\
\text { Systems }\end{array}$ & 105173 & $\begin{array}{c}\text { rabbit } \\
\text { polyclonal }\end{array}$ & $1: 100$ \\
\hline $\begin{array}{c}\text { Synaptotagmin } \\
7 \\
\end{array}$ & 7.239 & $\begin{array}{l}1.007 \\
\text { (ROV) } \\
\end{array}$ & 182.64 & 3.54 & $\begin{array}{l}\text { Synaptic } \\
\text { Systems }\end{array}$ & 105173 & $\begin{array}{c}\text { rabbit } \\
\text { polyclonal }\end{array}$ & $1: 250$ \\
\hline $\begin{array}{c}\text { Syndapin } \\
\text { (Pacsin) }\end{array}$ & 11.612 & $\begin{array}{l}0.493 \\
\text { (SEM) }\end{array}$ & 3201 & 131.28 & $\begin{array}{l}\text { Synaptic } \\
\text { Systems }\end{array}$ & 196002 & $\begin{array}{c}\text { rabbit } \\
\text { polyclonal }\end{array}$ & $1: 500$ \\
\hline
\end{tabular}




\begin{tabular}{|c|c|c|c|c|c|c|c|c|}
\hline SynGAP1 & 7.941 & $\begin{array}{l}1.278 \\
\text { (ROV) }\end{array}$ & 622.07 & 90.73 & $\begin{array}{l}\text { Thermo } \\
\text { Scientific }\end{array}$ & PA1-046 & $\begin{array}{c}\text { rabbit } \\
\text { polyclonal }\end{array}$ & $1: 1000$ \\
\hline Syntaxin1 & 16.991 & $\begin{array}{l}1.826 \\
\text { (SEM) }\end{array}$ & 20096 & 999.43 & $\begin{array}{l}\text { Synaptic } \\
\text { Systems }\end{array}$ & 110011 & $\begin{array}{c}\text { mouse } \\
\text { monoclonal }\end{array}$ & $1: 100$ \\
\hline Syntaxin13 & 13.925 & $\begin{array}{l}0.842 \\
\text { (SEM) }\end{array}$ & 157.83 & 3.49 & $\begin{array}{l}\text { Synaptic } \\
\text { Systems }\end{array}$ & 110131 & $\begin{array}{c}\text { mouse } \\
\text { monoclonal }\end{array}$ & $1: 100$ \\
\hline Syntaxin16 & 25.428 & $\begin{array}{l}1.991 \\
\text { (SEM) } \\
\end{array}$ & 91.27 & 5.68 & $\begin{array}{l}\text { Synaptic } \\
\text { Systems }\end{array}$ & 110162 & $\begin{array}{c}\text { rabbit } \\
\text { polyclonal }\end{array}$ & $1: 100$ \\
\hline Syntaxin16 & 12.975 & $\begin{array}{l}0.172 \\
\text { (ROV) }\end{array}$ & 91.27 & 5.68 & $\begin{array}{l}\text { Synaptic } \\
\text { Systems }\end{array}$ & 110162 & $\begin{array}{c}\text { rabbit } \\
\text { polyclonal }\end{array}$ & 1:100 \\
\hline Syntaxin2 & 12.214 & $\begin{array}{l}2.445 \\
\text { (SEM) } \\
\end{array}$ & $\begin{array}{l}\sim 100- \\
200 * *\end{array}$ & & $\begin{array}{l}\text { Synaptic } \\
\text { Systems }\end{array}$ & 110022 & $\begin{array}{c}\text { rabbit } \\
\text { polyclonal }\end{array}$ & $1: 100$ \\
\hline Syntaxin3 & 11.433 & $\begin{array}{l}4.084 \\
\text { (ROV) }\end{array}$ & $<100 * *$ & & $\begin{array}{l}\text { Synaptic } \\
\text { Systems }\end{array}$ & 110033 & $\begin{array}{c}\text { rabbit } \\
\text { polyclonal }\end{array}$ & $1: 100$ \\
\hline Syntaxin4 & 14.329 & $\begin{array}{l}5.247 \\
\text { (ROV) }\end{array}$ & $\begin{array}{l}100- \\
200 * *\end{array}$ & & $\begin{array}{l}\text { Synaptic } \\
\text { Systems }\end{array}$ & 110042 & $\begin{array}{c}\text { rabbit } \\
\text { polyclonal }\end{array}$ & $1: 100$ \\
\hline Syntaxin5 & 17.280 & $\begin{array}{c}0.66 \\
\text { (ROV) }\end{array}$ & $\begin{array}{l}100- \\
200 * *\end{array}$ & & $\begin{array}{l}\text { Synaptic } \\
\text { Systems }\end{array}$ & 110053 & $\begin{array}{c}\text { rabbit } \\
\text { polyclonal }\end{array}$ & $1: 100$ \\
\hline Syntaxin6 & 28.117 & $\begin{array}{l}3.863 \\
\text { (SEM) }\end{array}$ & 121.67 & 8.96 & $\begin{array}{c}\mathrm{BD} \\
\text { Bioscience } \\
\mathrm{s}\end{array}$ & 610636 & $\begin{array}{c}\text { mouse } \\
\text { monoclonal }\end{array}$ & $1: 500$ \\
\hline Syntaxin 7 & 55.981 & $\begin{array}{c}21.623 \\
(\mathrm{ROV})\end{array}$ & 78.6 & 4.45 & $\begin{array}{c}\text { Jahn } \\
\text { Departme } \\
\text { nt }\end{array}$ & & $\begin{array}{c}\text { mouse } \\
\text { monoclonal } \\
109.1\end{array}$ & $1: 100$ \\
\hline Syntaxin8 & 13.045 & $\begin{array}{l}5.342 \\
\text { (ROV) }\end{array}$ & $\begin{array}{l}\sim 100- \\
200 * *\end{array}$ & & $\begin{array}{l}\text { Synaptic } \\
\text { Systems }\end{array}$ & 110083 & $\begin{array}{c}\text { rabbit } \\
\text { polyclonal }\end{array}$ & $1: 100$ \\
\hline TfR & 21.293 & $\begin{array}{l}0.719 \\
\text { (SEM) } \\
\end{array}$ & $* * *$ & & Abcam & ab84036 & $\begin{array}{c}\text { rabbit } \\
\text { polyclonal }\end{array}$ & $1: 100$ \\
\hline TOM20 & 13.826 & $\begin{array}{l}0.379 \\
\text { (SEM) }\end{array}$ & 528.81 & 155.68 & $\begin{array}{l}\text { Sigma- } \\
\text { Aldrich }\end{array}$ & $\begin{array}{c}\text { WH000980 } \\
\text { 4M1 }\end{array}$ & $\begin{array}{c}\text { mouse } \\
\text { monoclonal }\end{array}$ & $1: 200$ \\
\hline Tubulin & 21.351 & $\begin{array}{l}1.367 \\
\text { (SEM) }\end{array}$ & 12056 & 615.3 & $\begin{array}{l}\text { Synaptic } \\
\text { Systems }\end{array}$ & 302203 & $\begin{array}{c}\text { rabbit } \\
\text { polyclonal }\end{array}$ & $1: 3000$ \\
\hline VAMP1 & 17.671 & $\begin{array}{l}1.315 \\
\text { (ROV) }\end{array}$ & 3884.3 & 181.95 & $\begin{array}{l}\text { Synaptic } \\
\text { Systems }\end{array}$ & 104002 & $\begin{array}{c}\text { rabbit } \\
\text { polyclonal }\end{array}$ & $1: 500$ \\
\hline VAMP2 & 21.158 & $\begin{array}{l}1.199 \\
\text { (SEM) }\end{array}$ & 26448 & 661.62 & $\begin{array}{l}\text { Synaptic } \\
\text { Systems }\end{array}$ & 104211 & $\begin{array}{c}\text { mouse } \\
\text { monoclonal }\end{array}$ & $1: 500$ \\
\hline VAMP2 & 29.226 & $\begin{array}{l}1.068 \\
\text { (SEM) }\end{array}$ & 26448 & 661.62 & $\begin{array}{l}\text { Synaptic } \\
\text { Systems }\end{array}$ & 104211 & $\begin{array}{c}\text { mouse } \\
\text { monoclonal }\end{array}$ & $1: 1000$ \\
\hline VAMP4 & 15.872 & $\begin{array}{l}0.792 \\
\text { (SEM) }\end{array}$ & 100.59 & 10.03 & $\begin{array}{l}\text { Synaptic } \\
\text { Systems }\end{array}$ & 136002 & $\begin{array}{c}\text { rabbit } \\
\text { polyclonal }\end{array}$ & $1: 100$ \\
\hline VAMP7 & 8.528 & $\begin{array}{l}0.924 \\
\text { (SEM) }\end{array}$ & $\begin{array}{l}\sim 100- \\
200 * *\end{array}$ & & Abcam & ab68776 & $\begin{array}{c}\text { rabbit } \\
\text { polyclonal }\end{array}$ & $1: 100$ \\
\hline vATPase & 129.622 & $\begin{array}{l}10.131 \\
\text { (SEM) }\end{array}$ & 742.37 & 32.97 & $\begin{array}{l}\text { Synaptic } \\
\text { Systems }\end{array}$ & 109002 & $\begin{array}{c}\text { rabbit } \\
\text { polyclonal }\end{array}$ & $1: 100$ \\
\hline VDAC1 & 19.532 & $\begin{array}{l}1.269 \\
\text { (SEM) } \\
\end{array}$ & 14422.99 & 720.71 & Santa Cruz & sc32063 & $\begin{array}{c}\text { rabbit } \\
\text { polyclonal }\end{array}$ & $1: 100$ \\
\hline Vglut1/2 & 13.518 & $\begin{array}{l}1.565 \\
\text { (SEM) }\end{array}$ & 8254.1 & 224.3 & $\begin{array}{l}\text { Synaptic } \\
\text { Systems }\end{array}$ & 135503 & $\begin{array}{c}\text { rabbit } \\
\text { polyclonal }\end{array}$ & $1: 100$ \\
\hline Vti1a & 12.390 & $\begin{array}{l}0.487 \\
\text { (SEM) }\end{array}$ & 50.55 & 2.51 & $\begin{array}{c}\text { BD } \\
\text { Bioscience } \\
\mathrm{s}\end{array}$ & 611220 & $\begin{array}{c}\text { mouse } \\
\text { monoclonal }\end{array}$ & $1: 100$ \\
\hline Vti1a & 17.383 & $\begin{array}{c}5.03 \\
\text { (SEM) }\end{array}$ & 50.55 & 2.51 & $\begin{array}{c}\mathrm{BD} \\
\text { Bioscience } \\
\mathrm{s}\end{array}$ & 611220 & $\begin{array}{c}\text { mouse } \\
\text { monoclonal }\end{array}$ & $1: 100$ \\
\hline
\end{tabular}

*based on Wilhelm et al, 2014 
**numbers estimated by the authors based on the copy numbers of functionally-related SNAREs, taking into account the high correlation between SNARE numbers in different pathways, as observed in the original work cited here

***not determined in the original work (Wilhelm et al, 2014), but currently under measurement in the Rizzoli laboratory for future references

Table 2 Numbers of primary antibodies per synaptosome for over 100 targets (continued) Additional information about the conditions of immunostainings.

\begin{tabular}{|c|c|c|}
\hline fixation & blocking & permeabilization \\
\hline 4\% PFA & $2.5 \% \mathrm{BSA}$ & $0.1 \%$ Triton $\mathrm{X}-100$ \\
\hline $4 \%$ PFA & $2.5 \% \mathrm{BSA}$ & $0.3 \%$ Tween 20 \\
\hline 3\% Glyoxal & $2.5 \% \mathrm{BSA}$ & $0.3 \%$ Tween 20 \\
\hline 4\% PFA & $2.5 \% \mathrm{BSA}$ & $0.1 \%$ Triton $\mathrm{X}-100$ \\
\hline $4 \%$ PFA & $2.5 \% \mathrm{BSA}$ & $0.1 \%$ Triton $\mathrm{X}-100$ \\
\hline 4\% PFA & $2.5 \% \mathrm{BSA}$ & $0.1 \%$ Triton $\mathrm{X}-100$ \\
\hline 4\% PFA & $2.5 \% \mathrm{BSA}$ & $0.1 \%$ Triton $X-100$ \\
\hline $4 \%$ PFA & $2.5 \% \mathrm{BSA}$ & $0.1 \%$ Triton $\mathrm{X}-100$ \\
\hline 3\% Glyoxal & $2.5 \% \mathrm{BSA}$ & $0.3 \%$ Tween 20 \\
\hline $4 \%$ PFA & $2.5 \% \mathrm{BSA}$ & $0.1 \%$ Triton $\mathrm{X}-100$ \\
\hline 4\% PFA & $2.5 \% \mathrm{BSA}$ & $0.1 \%$ Triton $X-100$ \\
\hline 4\% PFA & $2.5 \% \mathrm{BSA}$ & $0.1 \%$ Triton $\mathrm{X}-100$ \\
\hline 4\% PFA & $2.5 \% \mathrm{BSA}$ & $0.1 \%$ Triton $\mathrm{X}-100$ \\
\hline 3\% Glyoxal & $2.5 \% \mathrm{BSA}$ & $0.3 \%$ Tween 20 \\
\hline 4\% PFA & $2.5 \% \mathrm{BSA}$ & $0.1 \%$ Triton $X-100$ \\
\hline 3\% Glyoxal & $2.5 \% \mathrm{BSA}$ & $0.3 \%$ Tween 20 \\
\hline 4\% PFA & $2.5 \% \mathrm{BSA}$ & $0.1 \%$ Triton $\mathrm{X}-100$ \\
\hline 4\% PFA & $2.5 \% \mathrm{BSA}$ & $0.1 \%$ Triton $\mathrm{X}-100$ \\
\hline 3\% Glyoxal & $2.5 \% \mathrm{BSA}$ & $0.3 \%$ Tween 20 \\
\hline 3\% Glyoxal & $2.5 \% \mathrm{BSA}$ & $0.3 \%$ Tween 20 \\
\hline 3\% Glyoxal & $2.5 \%$ BSA & $0.3 \%$ Tween 20 \\
\hline 3\% Glyoxal & $2.5 \% \mathrm{BSA}$ & $0.3 \%$ Tween 20 \\
\hline 3\% Glyoxal & $2.5 \% \mathrm{BSA}$ & $0.3 \%$ Tween 20 \\
\hline 3\% Glyoxal & $2.5 \% \mathrm{BSA}$ & $0.3 \%$ Tween 20 \\
\hline 4\% PFA & $2.5 \% \mathrm{BSA}$ & $0.1 \%$ Triton $\mathrm{X}-100$ \\
\hline 4\% PFA & $2.5 \%$ BSA & $0.1 \%$ Triton $\mathrm{X}-100$ \\
\hline 4\% PFA & $2.5 \% \mathrm{BSA}$ & $0.1 \%$ Triton $\mathrm{X}-100$ \\
\hline 4\% PFA & $2.5 \% \mathrm{BSA}$ & $0.1 \%$ Triton X-100 \\
\hline 4\% PFA & $2.5 \% \mathrm{BSA}$ & $0.3 \%$ Tween 20 \\
\hline 4\% PFA & $2.5 \% \mathrm{BSA}$ & $0.1 \%$ Triton $\mathrm{X}-100$ \\
\hline 3\% Glyoxal & $2.5 \% \mathrm{BSA}$ & $0.3 \%$ Tween 20 \\
\hline 3\% Glyoxal & $2.5 \% \mathrm{BSA}$ & $0.3 \%$ Tween 20 \\
\hline 3\% Glyoxal & $2.5 \% \mathrm{BSA}$ & $0.3 \%$ Tween 20 \\
\hline 4\% PFA & $2.5 \% \mathrm{BSA}$ & $0.1 \%$ Triton $\mathrm{X}-100$ \\
\hline 4\% PFA & $2.5 \% \mathrm{BSA}$ & $0.1 \%$ Triton $\mathrm{X}-100$ \\
\hline 4\% PFA & $2.5 \% \mathrm{BSA}$ & $0.1 \%$ Triton $\mathrm{X}-100$ \\
\hline $4 \%$ PFA & $2.5 \% \mathrm{BSA}$ & 0.3\% Tween 20 \\
\hline
\end{tabular}




\begin{tabular}{|c|c|c|}
\hline 3\% Glyoxal & $2.5 \% \mathrm{BSA}$ & $0.3 \%$ Tween 20 \\
\hline 3\% Glyoxal & $2.5 \% \mathrm{BSA}$ & $0.3 \%$ Tween 20 \\
\hline 3\% Glyoxal & $2.5 \% \mathrm{BSA}$ & $0.3 \%$ Tween 20 \\
\hline 4\% PFA & $2.5 \% \mathrm{BSA}$ & $0.3 \%$ Tween 20 \\
\hline 3\% Glyoxal & $2.5 \% \mathrm{BSA}$ & $0.3 \%$ Tween 20 \\
\hline 3\% Glyoxal & $2.5 \% \mathrm{BSA}$ & $0.3 \%$ Tween 20 \\
\hline 3\% Glyoxal & $2.5 \% \mathrm{BSA}$ & $0.3 \%$ Tween 20 \\
\hline 4\% PFA & $2.5 \% \mathrm{BSA}$ & $0.1 \%$ Triton $\mathrm{X}-100$ \\
\hline 4\% PFA & $2.5 \% \mathrm{BSA}$ & $0.3 \%$ Tween 20 \\
\hline 4\% PFA & $2.5 \% \mathrm{BSA}$ & $0.1 \%$ Triton $\mathrm{X}-100$ \\
\hline 3\% Glyoxal & $2.5 \% \mathrm{BSA}$ & $0.3 \%$ Tween 20 \\
\hline 3\% Glyoxal & $2.5 \% \mathrm{BSA}$ & $0.3 \%$ Tween 20 \\
\hline 3\% Glyoxal & $2.5 \% \mathrm{BSA}$ & $0.3 \%$ Tween 20 \\
\hline 4\% PFA & $2.5 \% \mathrm{BSA}$ & $0.1 \%$ Triton $\mathrm{X}-100$ \\
\hline 4\% PFA & $2.5 \% \mathrm{BSA}$ & $0.1 \%$ Triton $\mathrm{X}-100$ \\
\hline 3\% Glyoxal & $2.5 \% \mathrm{BSA}$ & $0.3 \%$ Tween 20 \\
\hline 4\% PFA & $2.5 \% \mathrm{BSA}$ & $0.3 \%$ Tween 20 \\
\hline 3\% Glyoxal & $2.5 \% \mathrm{BSA}$ & $0.3 \%$ Tween 20 \\
\hline 4\% PFA & $2.5 \% \mathrm{BSA}$ & $0.3 \%$ Tween 20 \\
\hline 4\% PFA & $2.5 \% \mathrm{BSA}$ & $0.3 \%$ Tween 20 \\
\hline 4\% PFA & $2.5 \% \mathrm{BSA}$ & $0.3 \%$ Tween 20 \\
\hline 3\% Glyoxal & $2.5 \% \mathrm{BSA}$ & $0.3 \%$ Tween 20 \\
\hline 4\% PFA & $2.5 \% \mathrm{BSA}$ & $0.3 \%$ Tween 20 \\
\hline 3\% Glyoxal & $2.5 \% \mathrm{BSA}$ & $0.3 \%$ Tween 20 \\
\hline 4\% PFA & $2.5 \% \mathrm{BSA}$ & $0.1 \%$ Triton $\mathrm{X}-100$ \\
\hline 4\% PFA & $2.5 \% \mathrm{BSA}$ & $0.1 \%$ Triton $\mathrm{X}-100$ \\
\hline 4\% PFA & $2.5 \% \mathrm{BSA}$ & $0.1 \%$ Triton $\mathrm{X}-100$ \\
\hline 3\% Glyoxal & $2.5 \% \mathrm{BSA}$ & $0.3 \%$ Tween 20 \\
\hline 4\% PFA & $2.5 \% \mathrm{BSA}$ & $0.1 \%$ Triton $\mathrm{X}-100$ \\
\hline 4\% PFA & $2.5 \% \mathrm{BSA}$ & $0.1 \%$ Triton $\mathrm{X}-100$ \\
\hline 4\% PFA & $2.5 \% \mathrm{BSA}$ & $0.1 \%$ Triton $\mathrm{X}-100$ \\
\hline 3\% Glyoxal & $2.5 \% \mathrm{BSA}$ & $0.3 \%$ Tween 20 \\
\hline 3\% Glyoxal & $2.5 \% \mathrm{BSA}$ & $0.3 \%$ Tween 20 \\
\hline 4\% PFA & $2.5 \% \mathrm{BSA}$ & $0.1 \%$ Triton $\mathrm{X}-100$ \\
\hline 4\% PFA & $2.5 \% \mathrm{BSA}$ & $0.1 \%$ Triton $\mathrm{X}-100$ \\
\hline 3\% Glyoxal & $2.5 \% \mathrm{BSA}$ & $0.3 \%$ Tween 20 \\
\hline 4\% PFA & $2.5 \% \mathrm{BSA}$ & $0.1 \%$ Triton $\mathrm{X}-100$ \\
\hline 3\% Glyoxal & $2.5 \% \mathrm{BSA}$ & $0.3 \%$ Tween 20 \\
\hline 3\% Glyoxal & $2.5 \% \mathrm{BSA}$ & $0.3 \%$ Tween 20 \\
\hline 3\% Glyoxal & $2.5 \% \mathrm{BSA}$ & $0.3 \%$ Tween 20 \\
\hline 3\% Glyoxal & $2.5 \% \mathrm{BSA}$ & $0.3 \%$ Tween 20 \\
\hline 4\% PFA & $2.5 \% \mathrm{BSA}$ & $0.1 \%$ Triton $\mathrm{X}-100$ \\
\hline 3\% Glyoxal & $2.5 \% \mathrm{BSA}$ & $0.3 \%$ Tween 20 \\
\hline 4\% PFA & $2.5 \% \mathrm{BSA}$ & $0.1 \%$ Triton $\mathrm{X}-100$ \\
\hline 3\% Glyoxal & $2.5 \% \mathrm{BSA}$ & $0.3 \%$ Tween 20 \\
\hline
\end{tabular}




\begin{tabular}{|c|c|c|}
\hline 3\% Glyoxal & $2.5 \% \mathrm{BSA}$ & $0.3 \%$ Tween 20 \\
\hline 4\% PFA & $2.5 \% \mathrm{BSA}$ & $0.3 \%$ Tween 20 \\
\hline 4\% PFA & $2.5 \% \mathrm{BSA}$ & $0.1 \%$ Triton $\mathrm{X}-100$ \\
\hline 4\% PFA & $2.5 \% \mathrm{BSA}$ & $0.1 \%$ Triton $\mathrm{X}-100$ \\
\hline 4\% PFA & $2.5 \% \mathrm{BSA}$ & $0.1 \%$ Triton $\mathrm{X}-100$ \\
\hline 4\% PFA & $2.5 \% \mathrm{BSA}$ & $0.1 \%$ Triton $\mathrm{X}-100$ \\
\hline 4\% PFA & $2.5 \% \mathrm{BSA}$ & $0.1 \%$ Triton $\mathrm{X}-100$ \\
\hline 4\% PFA & $2.5 \% \mathrm{BSA}$ & $0.1 \%$ Triton $\mathrm{X}-100$ \\
\hline 3\% Glyoxal & $2.5 \% \mathrm{BSA}$ & $0.3 \%$ Tween 20 \\
\hline 3\% Glyoxal & $2.5 \% \mathrm{BSA}$ & 0.3\% Tween 20 \\
\hline 4\% PFA & $2.5 \%$ BSA & $0.1 \%$ Triton $\mathrm{X}-100$ \\
\hline 3\% Glyoxal & $2.5 \% \mathrm{BSA}$ & $0.3 \%$ Tween 20 \\
\hline 4\% PFA & $2.5 \% \mathrm{BSA}$ & $0.1 \%$ Triton $\mathrm{X}-100$ \\
\hline 3\% Glyoxal & $2.5 \% \mathrm{BSA}$ & $0.3 \%$ Tween 20 \\
\hline 4\% PFA & $2.5 \% \mathrm{BSA}$ & $0.1 \%$ Triton $\mathrm{X}-100$ \\
\hline 4\% PFA & $2.5 \% \mathrm{BSA}$ & $0.1 \%$ Triton $\mathrm{X}-100$ \\
\hline 4\% PFA & $2.5 \% \mathrm{BSA}$ & $0.1 \%$ Triton $\mathrm{X}-100$ \\
\hline 3\% Glyoxal & $2.5 \% \mathrm{BSA}$ & $0.3 \%$ Tween 20 \\
\hline 3\% Glyoxal & $2.5 \% \mathrm{BSA}$ & $0.3 \%$ Tween 20 \\
\hline 3\% Glyoxal & $2.5 \% \mathrm{BSA}$ & $0.3 \%$ Tween 20 \\
\hline 3\% Glyoxal & $2.5 \% \mathrm{BSA}$ & $0.3 \%$ Tween 20 \\
\hline 3\% Glyoxal & $2.5 \% \mathrm{BSA}$ & $0.3 \%$ Tween 20 \\
\hline 4\% PFA & $2.5 \% \mathrm{BSA}$ & $0.1 \%$ Triton $\mathrm{X}-100$ \\
\hline 4\% PFA & $2.5 \% \mathrm{BSA}$ & $0.3 \%$ Tween 20 \\
\hline 3\% Glyoxal & $2.5 \% \mathrm{BSA}$ & $0.3 \%$ Tween 20 \\
\hline 3\% Glyoxal & $2.5 \% \mathrm{BSA}$ & $0.3 \%$ Tween 20 \\
\hline 3\% Glyoxal & $2.5 \% \mathrm{BSA}$ & $0.3 \%$ Tween 20 \\
\hline 4\% PFA & $2.5 \% \mathrm{BSA}$ & $0.3 \%$ Tween 20 \\
\hline 3\% Glyoxal & $2.5 \% \mathrm{BSA}$ & $0.3 \%$ Tween 20 \\
\hline 4\% PFA & $2.5 \% \mathrm{BSA}$ & $0.1 \%$ Triton $\mathrm{X}-100$ \\
\hline 3\% Glyoxal & $2.5 \% \mathrm{BSA}$ & $0.3 \%$ Tween 20 \\
\hline 4\% PFA & $2.5 \% \mathrm{BSA}$ & $0.1 \%$ Triton $\mathrm{X}-100$ \\
\hline 3\% Glyoxal & $2.5 \% \mathrm{BSA}$ & $0.3 \%$ Tween 20 \\
\hline 4\% PFA & $2.5 \% \mathrm{BSA}$ & $0.1 \%$ Triton $\mathrm{X}-100$ \\
\hline 4\% PFA & $2.5 \% \mathrm{BSA}$ & $0.1 \%$ Triton $\mathrm{X}-100$ \\
\hline 4\% PFA & $2.5 \% \mathrm{BSA}$ & $0.1 \%$ Triton $\mathrm{X}-100$ \\
\hline 3\% Glyoxal & $2.5 \% \mathrm{BSA}$ & $0.3 \%$ Tween 20 \\
\hline 4\% PFA & $2.5 \% \mathrm{BSA}$ & $0.1 \%$ Triton $\mathrm{X}-100$ \\
\hline
\end{tabular}

\subsection{Discussion}

We introduced here an imaging-based method for the estimation of protein copy numbers, which can be implemented relatively easy in every lab equipped for standard immunohistochemistry experiments. CosiQuant relies on the comparison of immunolabeling 
signals from the sample of interest and biochemically characterized synaptosome preparations. In brief, we derive the protein copy numbers for POIs in samples of interest by comparing the immunostaining signals to those derived from synaptosome preparations with known copy numbers. Furthermore, we provide a substitute for this comparison (Fig. 4 and 5), so that other laboratories can apply the comparative imaging method to their sample of interest without having to use the exact same synaptosome preparations as used here.

In contrast to methods like Quantitative Western Blotting or mass spectrometry, no extensive purification steps are needed, and a much higher spatial resolution can be achieved. Furthermore, the use of synaptosomes instead of other standards, such as purified proteins or fluorescent beads, provides a more realistic comparison to neuronal samples. Effects such as the orientation of different proteins within membranes or molecular complexes, protein density, or steric hindrances, which all affect antibody binding, will apply in an identical fashion to both cultured synapses and synaptosomes, thus reducing the experimental differences between the standard sample and the sample to be measured.

Nevertheless, the comparative imaging approach also exhibits several limitations. A major one concerns the fact that CosiQuant assumes that the synaptosomes and the sample of interest are immunostained equally well. This should not be a major concern regarding the immunostaining of synaptosomes and of cultured neurons, since factors like penetration of antibodies should be comparable in both of these thin samples. For samples like whole brains or organs, however, this might differ substantially (although we found this not to be the case under optimal immunostaining conditions, for $\sim 20$ different antibodies (Wilhelm et al, 2014)). For such samples discrepancies in antibody penetration could be tested by staining for a GFP-tagged variant, whose fluorescence is used as a standard, and the differences in staining efficiency could be corrected for. Another procedure to ensure relatively equal staining conditions while comparing synaptosomes and whole brain/organ samples would be to prepare and to immunostain thin sections of the samples. In this fashion the sample depth can be defined, and can be limited to $\sim 10 \mu \mathrm{m}$. In order to achieve this, one can prepare cryosections of freshly dissected samples. Brains or organs can be either snap-frozen or chemically fixed first and then frozen in liquid nitrogen or on dry ice. Subsequently, the samples can be cut on a cryostat into $10 \mu \mathrm{m}$ thin sections, which can be immunostained on coverslips or glass slides.

Another limitation concerns the usage of numbers of single antibodies as a substitute to staining intensity for the synaptosome preparations. We provide here the numbers of single antibodies per synaptosome (Table 1 and 2) for over 100 different antibodies. But in order to use these numbers for the comparison of samples, one needs to stain the sample of interest under the exact same conditions (including fixation, antibody concentration, or blocking conditions) as the synaptosomes. These conditions might not be applicable to all samples. Furthermore, CosiQuant cannot be used for target proteins that have not been biochemically characterized yet in our synaptosomes. Nevertheless, the initial database of antibody numbers per synaptosome we provide here may already prove useful, since it covers pathways and networks ranging from the cytoskeleton to synaptic vesicle exo- and endocytosis, or to the postsynaptic density. Another limitation concerns the fact that 
antibody staining intensities may not sum linearly, and thus some estimates may not be correct. It has been our experience that this type of error is largely negligible, at least for structures that are not immensely bright. For example, immunostained single antibodies, which should be identified by $\sim 3$ secondary antibodies, are indeed $\sim 3$ fold brighter than individual secondary antibodies (Opazo et al, 2012; Truckenbrodt et al, 2018a), both in STED microscopy and in epifluorescence microscopy. This may not necessarily hold true in every imaging system, and should be tested carefully.

In summary, CosiQuant provides a comparably easy method for the estimation of protein numbers, and should in principle be applicable to a variety of different samples. This should be especially relevant in samples that are not purifiable (as cells from the sensory system or the peripheral nervous system (Scheffer et al, 2015; Matern et al, 2018) and thus cannot be analyzed by methods as mass spectrometry, which require abundant, purified samples.

\subsection{Methods}

\section{Synaptosome preparation}

Synaptosomes were purified by simple differential centrifugation and ficoll gradient centrifugation, as described previously in (Wilhelm et al, 2014). The synaptosome preparations from that study were snap frozen and stored at $-80^{\circ} \mathrm{C}$. The frozen synaptosomes were used by thawing them on ice and immobilization on glass coverslips (for details see immunocytochemistry section).

\section{Primary hippocampal neuron culture}

Rat primary hippocampal neuron cultures were prepared from newborn rats as described before (Beaudoin et al, 2012; Opazo et al, 2010) and were cultured under standard conditions. Cultured neurons of 10 to 15 days in vitro (21 or 22 DIV for the calculation of single antibody numbers per synapse; Fig. 5 and Table 1 and 2) were used for immunocytochemistry experiments.

\section{Immunocytochemistry of synaptosomes and primary hippocampal neurons}

For synaptosome immobilization on glass coverslips, the coverslips were coated with $5 \%$ bovine serum albumin (BSA, AppliChem \#A1391,0500) over night at $4^{\circ} \mathrm{C}$. Frozen synaptosome preparations were thawed on ice and dilutions (in PBS) were spun down in a centrifuge (VWR MegaStar3.0R) for $40 \mathrm{~min}$ at $4000 \mathrm{rpm}$, at $4^{\circ}$. Fixation of synaptosomes and cultured neurons was done with a $4 \%$ PFA solution for $15 \mathrm{~min}$ at $4^{\circ} \mathrm{C}$ and another $45 \mathrm{~min}$ at room temperature. Subsequently, preparations were washed briefly in PBS and quenched for $30 \mathrm{~min}$ in $100 \mathrm{mM} \mathrm{NH}{ }_{4} \mathrm{Cl}$. Permeabilization and background epitope blocking were achieved by 30 min incubation in PBS containing 0.1\% Triton X-100 and 5\% BSA (blocking solution). Both samples were incubated with primary antibodies diluted in blocking solution (prepared as one master mix) for $60 \mathrm{~min}$ at room temperature. After washing for $30 \mathrm{~min}$ in PBS the preparations were incubated with secondary antibodies, diluted in blocking solution 
for $60 \mathrm{~min}$ at room temperature. Primary and secondary antibodies used for all stainings, and the respective dilutions from a $1 \mathrm{mg} / \mathrm{ml}$ stock can be found in Table 3. Final washing in high-salt PBS (containing $500 \mathrm{mM} \mathrm{NaCl}$ ) and PBS was followed by embedding in Mowiol. The samples were imaged with an epifluorescent Nikon microscope.

Table 3 Antibodies used for the immunostaining of cultured neurons and synaptosomes

\begin{tabular}{|c|c|c|c|}
\hline target protein & species & company & dilution \\
\hline \multicolumn{4}{|l|}{ primary antibodies } \\
\hline Synaptophysin & guinea pig & SySy (\#101004) & $1: 300$ \\
\hline Bassoon & mouse & Enzo (ADI-VAM-PSO03-F) & $1: 100$ \\
\hline SNAP25 & rabbit & SySy (\#111002) & $1: 500$ \\
\hline Synapsin & rabbit & SySy (\#106002) & $1: 500$ \\
\hline Synaptogyrin & rabbit & SySy (\#103002) & $1: 200$ \\
\hline Syntaxin1 & rabbit & SySy (\#110302) & $1: 100$ \\
\hline Syntaxin12/13 & rabbit & SySy (\#110133) & $1: 200$ \\
\hline Synaptotagmin1 & rabbit & SySy (\#105102) & $1: 100$ \\
\hline VAMP2 & rabbit & SySy (\#104202) & $1: 500$ \\
\hline vGlut1/2 & rabbit & SySy (\#135503) & $1: 100$ \\
\hline \multicolumn{4}{|l|}{ secondary antibodies } \\
\hline anti-guinea pig IgG (Alexa488) & donkey & Dianova (\#706-545-148] & $1: 100$ \\
\hline anti-mouse IgG (Cy5) & donkey & Dianova (\#715-175-150) & $1: 100$ \\
\hline anti-rabbit IgG (Су3) & donkey & Dianova (\#711-165-152) & $1: 100$ \\
\hline
\end{tabular}

Some immunostainings of cultured neurons and synaptosomes used for calculating the number of single antibodies per synapse/synaptosome (Figure 5 and Table 1 and 2) were performed with slight variations to the protocol described above. Neurons cultured in the Banker arrangement (locally separated from the astrocyte feeder layer (Kaech \& Banker, 2006)) were fixed with a $3 \%$ glyoxal solution (Richter et al, 2018) and the blocking and permeabilization was achieved with $2.5 \%$ BSA and $0.3 \%$ Tween in PBS. Secondary antibody incubation was done for 60 min with Cy3-labeled donkey anti-mouse or rabbit Fab fragments (Dianova, \#715-166-150 and \#711-166-152) and Atto647N-labeled goat anti-mouse or rabbit antibodies (Rockland, \#610-156-121 and \#610-156-122). All other steps of the staining protocol remained the same.

The immunolabeling protocol for cultured neurons and synaptosomes stained for super resolution imaging (data used in Fig. 5 and Table 1 and 2) can be found in (Wilhelm et al, 2014).

Information about all antibodies used for stainings, in addition to the ones described in Table 3, can be found in Table 1 and 2 .

\section{Image acquisition}

Comparative imaging of the immunolabled synaptosomes and cultured neurons was done with an inverted epifluorescence Nikon Eclipse Ti-E microscope. The microscope was equipped with an HBO 100W lamp and images were acquired with an Andor IXON X3 897 
camera or a Nikon DS-Qi2 camera (for images used to calculate synaptosome staining signals in terms of number of antibodies, Table 1 and 2). The samples were imaged using a $100 \mathrm{X}$ PLAN APO oil immersion objective (NA 1.45). For multi-color imaging the following filter sets were used for Alexa488, Cy3 and Cy5 imaging: 470/40 nm (excitation, Alexa488), 525/50 nm (emission, Alexa488), 545/25 nm (excitation, Cy3), 605/70 nm (emission, Cy3), 620/60 nm (excitation, Cy5), 700/75 nm (emission, Cy5). Image acquisition software used was NiSElements AR (Nikon) and imaging parameters were kept the same for samples that were compared.

Super-resolution imaging (STED) and confocal imaging of neurons and synaptosomes (data used in Fig. 5 and Table 1 and 2) were performed with a Leica TCS SP5 STED microscope, exactly as described in (Wilhelm et al, 2014).

\section{Image analysis}

The image analysis was a two-step process carried out via custom-written Matlab (The Mathworks Inc.) routines. In the first step, the aim was to obtain initial guesses for the positions of the synapses. For this purpose, the script searches for the local intensity maxima in the images immunostained for synaptophysin (synapse marker). In order to avoid too many false positives caused by intensity noise, the images were filtered before searching for the local maxima using a Gaussian kernel with standard deviation $\sigma=480 \mathrm{~nm}$. In addition, intensity thresholds were applied, i.e. spots with very small peak intensities, which are most likely due to noise, were not taken into account. The thresholds were carefully chosen by eye, and they were set low enough so that real synapses were not accidentally removed. Thus at this point, due to the low thresholds, the set of selected synapses included a fraction of false positive synapse candidates. The set of synapse candidates was further filtered at a later stage of the image analysis.

In the second step, the script goes back to the raw (unfiltered) images and fits the exact positions, sizes and intensities for all synapse candidates using the initial guesses as obtained in the first step. For the fit a square region of interest $(2.2 \mu \mathrm{m} \times 2.2 \mu \mathrm{m})$ around each candidate is defined, and all candidates in all channels are fitted, i.e. those stained for synaptophysin, bassoon and the respective protein of interest (POI). The fit model is a 2D Gaussian function of variable size, position, orientation, amplitude and offset. For further analysis, mainly the total (integrated) intensity of each synapse candidate was used. The $\mathrm{R}^{2}$ between the model and the data was calculated as a measure for the goodness of the fit.

In a final step, all fitted synapse candidates were filtered according to the $\mathrm{R}^{2}$ value for each channel (synaptophysin, bassoon, POI). Candidates with a $R^{2}$ value below 0.85 (synaptophysin), 0.7 (bassoon) and 0.6 (POI) for the fit were discarded. Thus, sets of well fitted synapses and synaptosomes were left, which could be compared in terms of intensity. For display purposes only (Fig. 2a and Fig. 5) images were adjusted in brightness and contrast using ImageJ (Wayne Rasband, US National Institutes of Health). If intensities were compared, image adjustments in brightness and contrast were equally applied to all conditions. 


\section{Data analysis}

Intensities of the analyzed and filtered synapses and synaptosomes were collected and mean intensities were compared between neuron cultures and synaptosomes for each protein of interest. To analyze the signals in terms of antibody copy numbers, the intensities of the cellular structures (synaptosomes or cultured neurons) were divided by the average intensity of single antibodies immunostained on coverslips (thus equivalent to background antibody signals), as performed in the past (Opazo et al, 2012).

\section{Protocol for imaging single antibodies}

A simple procedure enables the analysis of the intensities of single immunostained primary antibodies. We typically rely on coverslips coated with Poly-L-lysine, which are incubated for 10-30 minutes with primary antibody dilutions (final concentrations of $10 \mu \mathrm{g} / \mathrm{ml}$ ), in PBS. The coverslips are then fixed, using the same fixation buffer as desired in the final application (for example $4 \%$ PFA, with or without $0.1 \%$ glutaraldehyde, or $3 \%$ glyoxal; see Richter et al, 2018, for further details on different fixation protocols). After fixation a quenching procedure is performed for $15-30$ minutes, with $50-100 \mathrm{mM} \mathrm{NH}_{4} \mathrm{Cl}$ in PBS, or $50-100 \mathrm{mM}$ glycine in PBS. This is followed by 2-3 rapid washes with PBS.

Alternatively, the fixation step can be avoided completely, as in most experiments the primary antibodies are not subjected to fixation during the immunostaining procedure. We only perform the fixation procedure when analyzing antibodies that are normally taken up by living cells, and therefore are fixed during the immunostaining process.

The coverslips are then incubated for 15-30 minutes with PBS containing 2-3\% BSA (blocking buffer). The blocking buffer coats the poly-L-lysine surface with BSA, and prevents the extensive binding of secondary antibodies to this surface. This is followed by incubating the coverslips with secondary antibodies (diluted to $10 \mu \mathrm{g} / \mathrm{ml}$ ) in PBS containing 2-3\% BSA, for 30-60 minutes. The coverslips are then washed extensively: $3 \times 5$ minutes with PBS containing high salt $(500 \mathrm{mM} \mathrm{NaCl})$, and $3 \times 5$ minutes with normal PBS (150 mM NaCl). The coverslips can then be mounted in the desired mounting medium, and can be imaged.

For the image analysis, we recommend applying a bandpass filter on the images, to detect the antibody spots, followed by Gaussian fits on the spots, to obtain the total signal intensity associated to each spot. The population of antibody intensities obtained should be fitted well by a single Gaussian, whose peak position indicates the average single primary antibody intensity. Performing this experiment with super-resolution is very convenient, since then large spots (full width at half maximum, FWHM, larger than $50 \mathrm{~nm}$ ) can be discounted. They do not represent single antibodies, but presumably are caused by dirt on the coverslips.

Importantly, a very simple and practical application for obtaining this type of value, without any additional experiments, has been to investigate the background spots obtained on the clean coverslip areas adjacent to cultured neurons, in the normal immunostaining experiments used for determining protein intensities in the cultured neurons. It has been our experience that the results obtained are indistinguishable from those obtained when immunostaining antibodies on coverslips in separate experiments, as described above, provided that large spots, indicating dirt or cell debris on the coverslips, are discounted. 
Nevertheless, a number of experiments in which antibodies on coverslips are measured as indicated above should be performed, to test that the background spots from the cultures can be indeed trusted, since inappropriate handling of the cultures (poor fixation or blocking, for example) may result in the formation of extensive antibody clusters, which would perturb the measurements.

\section{Statistics}

Bars and data points in Figure 3 and 5 show mean values. All error bars represent the standard error of the mean (SEM), calculated in Sigma Plot (Systat Software, Inc.), unless stated otherwise in the figure legend.

Column C in Table 1 shows the standard error of the mean (SEM) from typically several hundreds of analyzed synapses or the range of value (ROV) derived from several experiments, which were done by multiple investigators.

\section{Animals}

Wild type Wistar rats (Rattus norvegicus) for the preparation of primary hippocampal neuron cultures and synaptosomes were obtained from the University Medical Center Göttingen. All animals were handled according to the specifications of the University of Göttingen and of the local authority, the State of Lower Saxony (Landesamt für Verbraucherschutz, LAVES, Braunschweig, Germany). All animal experiments were approved by the local authority, the Lower Saxony State Office for Consumer Protection and Food Safety (Niedersächsisches Landesamt für Verbraucherschutz und Lebensmittelsicherheit). 
$05 \mid$ The molecular Anatomy of synaptic vesicle recycling at the inner hair cell ribbon synapse 


\subsection{Introduction}

The improvement of existing fixation methods and the establishment of a new technique to estimate protein copy numbers, which I have described in the previous two chapters, can now be applied to IHC investigations of the synaptic vesicle recycling process. Implementing those two novelties in IHC sample preparation and analysis, enables me to overcome, at least to a certain extent, the difficulties that have been associated with investigations of IHC ribbon synapses, i.e. insufficient immunostaining quality and lack of a suitable quantification method. These have been major reasons for the lack of in depth studies of many synaptic proteins in the IHC ribbon synapse in the past. A few proteins, like otoferlin, ribeye and vGlut3, have been studied in more detail, mainly by investigating respective gene knock-outs in mice. Otoferlin is the most prominent and well-studied protein in IHCS, due to its unique expression in those cells. It has been found to be involved in $\mathrm{Ca}^{2+}$ mediated synaptic vesicle fusion, by studying a defect of this protein in a form of human deafness (Roux et al, 2006). It has also been suggested numerous times that otoferlin substitutes for synaptotagmin 1 as calcium sensor in IHCS, since it has been discovered that synaptotagmin 1 (the calciumsensor of conventional synapses) is not expressed in mature IHCS and otoferlin has the ability to bind $\mathrm{Ca}^{2+}$ via its $\mathrm{C}_{2}$ domains (Beurg et al, 2010; Reisinger et al, 2011; Johnson \& Chapman, 2010; Michalski et al, 2017; Dulon et al, 2009). This is in line with the observation that otoferlin expression replaces synaptotagmin 1 expression in IHCS during development around the onset of hearing (Michanski et al, 2019). Additionally, otoferlin has been reported to play a role in the priming process of synaptic vesicles and in synaptic vesicle replenishment (Michalski et al, 2017; Pangršič et al, 2010), suggesting that otoferlin exhibits versatile functions in IHCS. Already studied to a much lesser extent than otoferlin is the protein vGlut3, which is the glutamate transporter of synaptic vesicles in IHCs. It mediates the filling of synaptic vesicles with neurotransmitter and again has mainly been characterized through gene knock-out studies (Ruel et al, 2008; Seal et al, 2008). Due to the fact that both proteins, otoferlin and vGlut3, are almost exclusively expressed in IHCS and are present throughout the whole cytosol, they have been used as IHC markers in immunostainings of the Organ of Corti (Revelo et al, 2014; Seal et al, 2008; Pangršič et al, 2010). Naturally, the ribbon, more precisely the protein ribeye, has been used as a marker for the synapse in IHCs (Uthaiah \& Hudspeth, 2010; Ruel et al, 2008; Frank et al, 2010). Ribeye consists of an A-domain, which is responsible for the ribbon assembly and structure through oligomerization (Magupalli et al, 2008) and a B-domain, which exhibits high sequence similarity to the transcriptional repressor CtBP2 (C-terminal binding protein 2; Rutherford \& Pangršič, 2012). The B domain has also been proposed to play a role in tethering synaptic vesicles to the ribbon (Schmitz et al, 2000; Schmitz, 2009). Both domains can be targeted by antibodies for the immunolabeling of ribeye as synaptic marker.

Some studies attempted to investigate the molecular composition of ribbon synapses beyond the commonly known proteins mentioned above, but all of them face major problems associated with the use of the respective method in IHCS (i.e. immunofluorescence 
studies, electron microscopy studies, biochemical assays or transcriptomics). As mentioned before, a common approach to investigate the function of a specific protein is to use specific knock-out mouse strains and to study the effects of the absence of the protein. This becomes very labor-intensive when investigating the entirety of proteins involved in a certain process, like the synaptic vesicle recycling process, especially because there is only a small amount of specific knock-out mutants available for IHC studies. Additionally, effects can be masked by proteins with redundant functions to the target protein, since these proteins might compensate for the loss of the genetically ablated protein. Therefore, other approaches have been tried to investigate the proteins in IHCS, especially proteins associated with the ribbon synapse. One study of Uthaiah and Hudspeth in 2010 investigated the presence and absence of a variety of proteins in chicken and mouse cochlea using mass spectrometry along with immunoprecipitation methods and immunofluorescent imaging (Uthaiah \& Hudspeth, 2010). These experiments identified many proteins which might be relevant for the synaptic vesicle recycling process, including conventional exocytosis SNARE proteins. Unfortunately, the sample preparation and purification methods they used, are prone to contain contaminations from other synapses (Rutherford \& Pangršič, 2012) and at least for mouse preparations they also used samples from pre-hearing developmental stages, not taking into account that protein expression changes during that time. There are other studies, implementing immunofluorescent imaging of synaptic proteins in IHCs, like AP2 (Duncker et al, 2013) or rab3, syntaxin6 and syntaxin16 (Revelo et al, 2014). Nevertheless, these studies do not give an estimate for the abundance of these proteins and are focusing only on one or a few proteins. In case of the latter study, immunostainings of the POIs were combined with an IHC marker staining but location of the POls were not assessed in relation to the ribbon synapse (Revelo et al, 2014). There is one additional superresolution study, investigating the ribbon synapse, but it focuses mainly on the distribution of $\mathrm{Ca}^{2+}$ channels (Rutherford, 2015). Imaging the IHC ribbon synapse with even higher resolution, provided by electron microscopy, is usually used to investigate the structure of the ribbon synapse rather than the identification of proteins (Michanski et al, 2019), because it proves to be even more difficult to specifically stain proteins and determine the molecular identity of structures in EM.

Additionally, investigations of protein expression and abundance with methods providing higher throughput, like MS and transcriptomic analyses, have been conducted on IHCS (Kantardzhieva et al, 2012; Hickox et al, 2017; Liu et al, 2014b; Scheffer et al, 2015). But, in addition to already mentioned issues of insufficient sample purity, the proteins identified in those studies largely comprise components of the cytoskeleton or the transcription and translation machinery, rather than synaptic proteins. Components of the synaptic vesicle recycling machinery have been identified only rarely and if so, then often in contradicting results between different studies. Furthermore, transcriptomic investigations might not represent a direct image of protein abundance, due to the expression of splice variants, post-translational modifications and different protein turnover rates (Hickox et al, 2017).

An issue that has been investigated with special interest and which illustrates the finding of contradicting results in different studies, is the expression of conventional neuronal 
exocytosis SNARE proteins in IHCs. Some studies have reported the presence of syntaxin 1, SNAP25 and VAMP2 at the hair cell ribbon synapse, using various methods (Uthaiah \& Hudspeth, 2010; Wenthold et al, 2002; Safieddine \& Wenthold, 1999). In addition to the detection in mass spectrometry analysis by Uthaiah and Hudspeth (mentioned above), Safieddine and Wenthold detected RNA and protein expression of syntaxin 1, SNAP25 and VAMP1/2 in rat and guinea-pig cochlear hair cells by reverse transcription PCR, in situ hybridization and immunohistochemistry (Safieddine \& Wenthold, 1999). In contrast, the investigations by Nouvian and colleagues, using botulinum neurotoxin to block the function of these SNARE proteins, showed exocytosis despite the inhibition, measured by electrophysiology experiments (Nouvian et al, 2011). Exocytosis in genetically SNAREdeficient mice was also reported to be normal. Furthermore, they were not able to detect syntaxin 1, SNAP25 and VAMP1/2 in immunostainings of IHCs, although mRNA was detected by real-time PCR (Nouvian et al, 2011). These contradicting results of both studies are still controversially discussed. On the one hand does the absence of an effect upon SNARE inhibition/ablation not necessarily mean that these SNARE proteins are not expressed. Their inhibition/absence might be compensated by additional proteins with redundant functions. On the other hand, Nouvian and colleagues, using an advanced imaging approach comparted to Safieddine, provided the localization of proteins by co-staining with IHC markers. These advanced imaging experiments located SNARE signal only in efferent innervations of the IHCS. Thus, the question, whether conventional neuronal exocytosis SNARE proteins are expressed and necessary for synaptic vesicle fusion in IHCS, remains insufficiently answered. In conclusion, none of the studies above was able to provide a good overview of the molecular composition of the ribbon synapse, which at the same time is detailed enough to make assumptions about the functional processes at that synapse. Therefore, I intended to create a better picture of the protein composition at the hair cell ribbon synapse, by determining the location and abundance of synaptic proteins possibly involved in the vesicle recycling process at the ribbon synapse. Using an improved chemical fixation method for the immunolabeling of synaptic proteins in IHCs and a new technique to estimate protein copy numbers, I was able to investigate 19 proteins, providing a preliminary model of their spatial organization and abundance at the IHC ribbon synapse.

\subsection{Materials and Methods}

\subsubsection{Dissection of the Organ of Corti}

Organs of Corti were dissected freshly from wildtype mice of the substrain C57BI/6J at the age of P14 - P18. For this, mice were anaesthetized and decapitated. The inner ear was taken out and the apex of the Organ of Corti was dissected in cold HEPES-buffered HBSS (5.36 mM KCl, $141.7 \mathrm{mM} \mathrm{NaCl}, 10 \mathrm{mM}$ HEPES, $34 \mathrm{mM}$ L-glutamine, $6.9 \mathrm{mM}$ D-glucose, $1 \mathrm{mM}$ $\mathrm{MgCl}_{2}, 0.5 \mathrm{mM} \mathrm{MgSO}_{4}, \mathrm{pH}$ 7.4) without calcium to ensure a resting state of the cells. After dissection, the organs were chemically fixed either with a 4\% PFA solution or a $3 \%$ glyoxal (Richter et al, 2018) solution for 30 min on ice and another $30 \mathrm{~min}$ at RT. 


\subsubsection{Sample preparation and cryosectioning of the Organ of Corti}

After fixation, the organs were briefly washed with PBS $(137 \mathrm{mM} \mathrm{NaCl}, 2.7 \mathrm{mM} \mathrm{KCl}, 10 \mathrm{mM}$ $\mathrm{Na}_{2} \mathrm{HPO}_{4}, 2 \mathrm{mM} \mathrm{KH} \mathrm{PO}_{4}, \mathrm{pH}$ 7.4) and quenched in $100 \mathrm{mM} \mathrm{NH}{ }_{4} \mathrm{Cl}$ for 30 min at RT. After another brief washing in PBS, the organs were embedded in low gelling temperature agarose (Sigma, \#A9414) containing graphite to create contrast between the black agarose and the white organ for better orientation while cryosectioning. The agarose block containing the Organ of Corti was then embedded in Tissue-Tek O.C.T. (Sakura Finetec Europe B. V., Alphen aan den Rijn, The Netherlands) and frozen on dry ice. The samples were kept at $-80^{\circ} \mathrm{C}$ until cryosectioning (at least over night).

The samples were cut on a Leica CM1850 cryotome into $10 \mu \mathrm{m}$ thin sections. The sections were transferred to glass microscope slides (Superfrost Plus from Thermo Scientific, \#J1800AMNZ) and kept at $4{ }^{\circ} \mathrm{C}$ until immunohistochemistry.

\subsubsection{Immunohistochemistry}

Immunostaining of the cryosections for antibody screening and for super resolution imaging (localization and protein copy number estimation) followed the same protocol, but with differences in the choice of secondary antibodies.

\section{Antibody screening}

Cryosections of the Organ of Corti were stained on the glass microscope slides. The already fixed and quenched slices were permeabilized and background epitopes were blocked for 3 times 10 min with a blocking solution containing 0.1\% Triton X-100 and 2\% Trypton-Pepton in PBS. Primary antibodies were applied for $60 \mathrm{~min}$ at RT and diluted in the blocking solution (for an overview of used antibodies see Table 1). Subsequent washing for 3 times 10 min with blocking solution ensured the elimination of unbound antibodies and was followed by incubation of the slices in secondary antibodies (diluted in blocking solution as well) for 60 min at RT in a humidified dark chamber. The following secondary antibodies were used to label the respective primary antibodies: donkey anti-mouse coupled to Cy2 (Dianova \#715225-150), donkey anti-rabbit coupled to Cy2 (Dianova \#711-225-152), donkey anti-mouse coupled to Cy3 (Dianova \#715-165-150) and donkey anti-rabbit coupled to Cy3 (Dianova \#711-165-152). Secondary antibody incubation was followed by several washing steps with high salt PBS (500 mM NaCl, 3 times $10 \mathrm{~min}$ ) and PBS (2 times $10 \mathrm{~min}$ ). Finally, the slices were embedded in mowiol and kept at $4{ }^{\circ} \mathrm{C}$ until imaging. 
Table 1 Primary antibodies used for antibody screening and super-resolution imaging

\begin{tabular}{|c|c|c|c|}
\hline target protein & species & company & dilution \\
\hline Amphiphysin & rabbit & SySy (\#120002) & $1: 400$ \\
\hline AP180 & rabbit & SySy (\#155003) & $1: 500$ \\
\hline AP2 & mouse & $\mathrm{BD}(\# 610501)$ & $1: 100$ \\
\hline Bassoon & mouse & Enzo (\#ADI-VAM-PS003) & $1: 100$ \\
\hline Bassoon & rabbit & SySy (\#141002) & $1: 500$ \\
\hline Clathrin HC & rabbit & abcam (\#21679) & $1: 200$ \\
\hline Clathrin LC & mouse & SySy (\#113011) & 1:100 \\
\hline CSP & rabbit & SySy (\#154003) & $1: 500$ \\
\hline CtBP2 & mouse & BD (\#612044) & $1: 100$ \\
\hline Dynamin & mouse & BD (\#610245) & $1: 100$ \\
\hline Endophilin & rabbit & SySy (\#159002) & $1: 200$ \\
\hline GFP & mouse & Invitrogen (\#A11120) & $1: 50$ \\
\hline Munc18 & rabbit & SySy (\#116003) & $1: 500$ \\
\hline Otoferlin & mouse & abcam (\#53233) & $1: 350$ \\
\hline Rab3a & mouse & BD (\#610379) & $1: 100$ \\
\hline Ribeye & rabbit & SySy (\#192003) & $1: 1500$ \\
\hline RIM1 & rabbit & SySy (\#140003) & $1: 200$ \\
\hline RIM2 & mouse & $\begin{array}{c}\text { Novus (\#H0000-9699- } \\
\text { M01a) }\end{array}$ & 1:100 \\
\hline SCAMP1 & rabbit & SySy (\#121002) & $1: 1000$ \\
\hline SNAP23 & rabbit & SySy (\#111202) & $1: 100$ \\
\hline SNAP25 & mouse & SySy (\#111011) & $1: 200$ \\
\hline SNAP29 & rabbit & SySy (\#111302) & $1: 300$ \\
\hline SNAP47 & rabbit & SySy (\#111403) & $1: 100$ \\
\hline Synaptogyrin & rabbit & SySy (103002) & $1: 200$ \\
\hline Synaptojanin & rabbit & SySy (\#145003) & $1: 100$ \\
\hline Synaptophysin & rabbit & Reinhard Jahn & $1: 500$ \\
\hline Synaptophysin & guinea pig & SySy (\#101004) & $1: 300$ \\
\hline Synaptotagmin 2 & rabbit & SySy (\#105123) & $1: 500$ \\
\hline Synaptotagmin 7 & rabbit & SySy (\#105173) & $1: 200$ \\
\hline Syndapin & rabbit & SySy (\#196002) & $1: 500$ \\
\hline Syntaxin1 & mouse & abcam (\#3265) & $1: 100$ \\
\hline Syntaxin12/13 & rabbit & SySy (\#110133) & $1: 250$ \\
\hline Syntaxin16 & rabbit & SySy (\#110162) & $1: 200$ \\
\hline Syntaxin2 & rabbit & SySy (\#110022) & $1: 100$ \\
\hline Syntaxin3 & rabbit & SySy (\#110033) & $1: 100$ \\
\hline Syntaxin4 & rabbit & SySy (\#110042) & 1:100 \\
\hline Syntaxin5 & rabbit & SySy (\#110053) & $1: 200$ \\
\hline Syntaxin6 & mouse & BD (\#610636) & 1:100 \\
\hline Syntaxin7 & rabbit & SySy (\#110073) & 1:100 \\
\hline Syntaxin8 & rabbit & SySy (\#110083) & $1: 200$ \\
\hline VAMP1/2/3 & rabbit & SySy (\#104203) & $1: 200$ \\
\hline VAMP2 & rabbit & SySy (\#104202) & 1:500 \\
\hline
\end{tabular}




\begin{tabular}{|c|c|c|c|}
\hline VAMP3 & rabbit & Novus (\#NB100-91353) & $1: 100$ \\
\hline VAMP4 & rabbit & SySy (\#136002) & $1: 250$ \\
\hline VAMP7 & rabbit & SySy (\#232003) & $1: 500$ \\
\hline VAMP8 & rabbit & SySy (\#104303) & $1: 250$ \\
\hline vATPase & rabbit & SySy (\#109002) & $1: 100$ \\
\hline vGlut3 & rabbit & SySy (\#135203) & $1: 500$ \\
\hline vGlut3 & guinea pig & SySy (\#135204) & $1: 500$ \\
\hline Vti1a & mouse & BD (\#611220) & $1: 100$ \\
\hline
\end{tabular}

Indicated antibody was a kind gift of Prof. Dr. Reinhard Jahn, Max Planck Institute for Biophysical Chemistry, Göttingen, Germany.

Localization and protein copy number estimation

For the estimation of protein copy numbers, Organ of Corti cryosections were stained in parallel with synaptosome preparations. The same samples were used for super-resolution imaging to localize proteins of interest.

In order to stain synaptosome preparations in parallel with the Organ of Corti slices, frozen synaptosomes (same preparations as described in chapter 04) were thawed on ice and plated on BSA coated coverslips by centrifugation for $40 \mathrm{~min}$ at $4000 \mathrm{rpm}$ at $4^{\circ} \mathrm{C}$ in a VWR MegaStar3.0R centrifuge. Synaptosomes were fixed like the Organs of Corti with either $4 \%$ PFA or $3 \%$ glyoxal for $30 \mathrm{~min}$ on ice and another $30 \mathrm{~min}$ at RT. Fixation was followed by a brief wash with PBS and quenching for $30 \mathrm{~min}$ with $100 \mathrm{mM} \mathrm{NH}_{4} \mathrm{Cl}$ at RT. The samples (synaptosomes and cryosections of the Organ of Corti) were then immunostained in parallel following the protocol described above. 3-colour stainings were performed on each sample (protein of interest and 2 marker proteins) and in order to image them with a STED microscope, the following secondary antibodies coupled to appropriate fluorophore dyes have been used (for an overview of used primary antibodies see Table 1): donkey anti-guinea pig coupled to Alexa488 (Dianova \#706-545-148), goat anti-mouse coupled to star580 (Abberior, \#ST580-1001-500UG), goat anti-rabbit coupled to star635P (Abberior, \#ST635P1002-500UG), goat anti-rabbit coupled to star580 (Abberior, \#ST580-1002-500UG), goat anti-mouse coupled to star635P (Abberior, \#ST635P-1001-500UG), as well as donkey antimouse coupled to Alexa488 (Dianova \#715-545-151) and goat anti-guinea pig coupled to star580 (Abberior \#ST580-1006-500UG).

\subsubsection{Imaging}

\section{Antibody screening}

Imaging of immunolabled cryosections for the antibody screening was done with an inverted epifluorescence Nikon Eclipse Ti-E microscope (the same as described in chapter 03 and 04), The samples were imaged using a 100X PLAN APO oil immersion objective (NA 1.45) and the following filter sets were used for Cy2 and Cy3 imaging: 470/40 nm (excitation, Cy2), 525/50 $\mathrm{nm}$ (emission, Cy2), 545/25 nm (excitation, Cy3), 605/70 nm (emission, Cy3). The image acquisition software used was NiS-Elements AR (Nikon). 
Localization and protein copy number estimation

The cryosections immunostained for protein copy number estimation and localization were imaged using a Quad scan STED microscope (Abberior Instruments), built on the basis of an Olympus confocal microscope (IX83). The setup was equipped with a $775 \mathrm{~nm}$ pulsed STED lasers, which was excited by two pulsed diode laser sources (561 nm and $640 \mathrm{~nm}$ ). For confocal imaging, a continuous wavelength laser $(488 \mathrm{~nm})$ and the two pulsed diode lasers (561 $\mathrm{nm}$ and $640 \mathrm{~nm}$ ) were used for excitation. Detection was achieved by avalanche photodiodes. All samples were imaged using a 100X UPlanS APO oil immersion STED objective (NA 1.4). Imaging software used was the Imspector Data Acquisition and Analysis Software purchased with the setup from Abberior Instruments. Images were acquired with a pixel size of $15 \mathrm{~nm}$ for STED images and $50 \mathrm{~nm}$ for confocal images.

\subsubsection{Analysis}

All analyses were done with Matlab (The Mathworks Inc.) using custom-written scripts described below.

\section{Antibody screening}

For the evaluation of antibodies used to stain different synaptic proteins in IHCs, the respective staining signal was correlated to the signal from an IHC marker (otoferlin or vGlut3). This was calculated automatically in Matlab. The correlation coefficient between the staining signal from the $\mathrm{POI}$ and the IHC marker were calculated for the area of the IHC as well as for the whole image (including areas of other cell types not stained by the IHC marker). The product of these two correlation coefficients was then compared to a positive and negative control. For statistical analyses, the two-sided student's t-test (unpaired) was used in case of normal distribution of the data sets or Wilcoxon rank sum test was applied, in case one or both data sets did not show normal distribution.

Localization and protein copy number estimation

Confocal and STED images used for the localization and estimation of protein copy numbers were manually processed prior to automatic analysis in Matlab. Areas from the confocal and STED images of both samples (IHCS and synaptosomes) were selected by hand in order to eliminate imaging artifacts. All subsequent analyses were done on those pre-selected image areas. Based on the signal of the synapse marker staining (ribeye/CtBP2 for IHCs and bassoon for synaptosomes), single spots were automatically identified as synapses via thresholding in Matlab.

For the localization of synaptic proteins within the ribbon synapse, the images of each synapse were fitted to a superficial ribbon synapse model, so the orientation of each imaged synapse was roughly the same and the signal distribution of the stained POI and marker proteins could be averaged.

For the estimation of protein copy numbers from staining signals, the average intensities of the POI stainings in the selected synapse areas were extracted. This was done based on the confocal images only, in order to ensure a linear relation between signal intensity and 
number of labeled structures. Intensity values from non-specific control stainings were substracted for each POI, respectively. The average intensity values for each POI were multiplied by the number of pixels of the measured area for IHCs and respective synaptosome sample (stained and imaged in parallel). The average signal for each POI in IHC synapses was expressed as fold over synaptosome and based on the resulting ratio and the known copy numbers of the POIs in synaptosome samples (Wilhelm et al, 2014), the average copy numbers in IHC ribbon synapses were calculated.

\section{$3 \mathrm{D}$ reconstructions}

3-dimensional reconstructions of the IHC ribbon synapse were based on electron microscopy data, kindly provided by Prof. Dr. Carolin Wichmann (University of Göttingen Medical Center). Apical cochlear samples were taken from wildtype mice (P14, inhibited synapses) and were prepared as described in Chapochnikov et al, 2014. Serial sections (60 nm thickness) were prepared from those high pressure frozen and freeze-substituted samples using a UC7 microtome (Leica Microsystems). IHC ribbon synapses were imaged with a JEOL electron microscope (JEM 1011).

The images of the EM sections were aligned manually and the different organelles of the ribbon synapse were traced by hand in Matlab. The following features were traced: the synaptic ribbon, the plasma membrane, the active zone, vacuoles and synaptic vesicles. The coordinates were saved and can later be used as a basis for modeling the investigated synaptic proteins within the ribbon synapse.

\subsubsection{Animals}

Wild type mice (Mus musculus) of either sex from the substrain C57BI/6J were obtained from the group of Prof. Dr. Michael Müller, University Medical Center Göttingen.

All animals were handled according to the specifications of the University of Göttingen and of the local authority, the State of Lower Saxony (Landesamt für Verbraucherschutz, LAVES, Braunschweig, Germany).

\subsection{Results}

\subsubsection{Antibody screening for the immunostaining of synaptic proteins in IHCs}

In order to investigate various synaptic proteins in the IHC ribbon synapse via imaging techniques, I first had to test whether available antibodies against those target proteins are working reliably (i.e. labeling the targets specifically) in IHCs. This is far from trivial, since most available antibodies against synaptic proteins are optimized for the use in hippocampal neuron cultures or brain slices, mostly consisting of conventional synapses. Therefore, I established a relatively easy screening for antibodies, that can be used in IHCs, allowing me to test various antibodies against several different synaptic proteins. I immunostained $10 \mu \mathrm{m}$ thin cryosections of the Organ of Corti for an IHC marker (Otoferlin or vGlut3) and the protein of interest (POI). This way I was able to test several different antibodies on different 
cryosections of the same organ, allowing for a higher throughput. Since I could show previously that glyoxal fixation improves the immunostaining of several targets, I tested the antibodies in PFA and glyoxal fixed cryosections and selected the method that worked best for the individual target protein. To evaluate which antibody returns a reliable labeling signal, I performed a simple correlation test. I calculated the correlation coefficient of the signals originating from the IHC marker staining and the POI staining. I compared those correlation coefficients to a positive and negative control staining. The positive control comprises the labeling of otoferlin (IHC marker) with one primary antibody, which was detected by two differently labeled secondary antibodies. This results in a correlation coefficient of $\sim 85 \%$. The negative control staining contained the labeling of GFP by an antiGFP antibody in addition to the IHC marker labeling. Since GFP is not expressed in the wildtype IHCS, this gives an estimate of how much correlation results from non-specific staining. Examples of such immunostainings can be seen in Figure $1 \mathrm{~A}$. Evaluation of the antibodies used for the staining of 42 POls was done by comparison of correlation coefficients from POI stainings to negative control stainings (Figure 1B). Only antibodies that showed reasonable staining signals by visual inspection in a first trial staining were subjected to this screening. The difference to the correlation coefficient resulting from the negative control (red line in Figure 1B) was tested for each POI and only antibodies against target proteins that resulted in a significant higher correlation to the IHC marker were taken for further analysis.

For the initial selection of POIs I was mainly focusing on proteins known to be involved in the exo- and endocytosis of vesicles. An additional criterion for the selection of target proteins was the availability of protein copy numbers in biochemically characterized synaptosomes from a previous study (Wilhelm et al, 2014) in order to estimate protein copy numbers in IHCS at a later timepoint. In total, I was able to identify 19 proteins that seem to be expressed in IHCS and were detectable by commonly available antibodies. Amongst these proteins was synaptophysin, which is known to not be expressed in IHC ribbon synapses (GilLoyzaga \& Pujol, 1988; Safieddine \& Wenthold, 1997). Nevertheless, it is expressed in neighboring efferent synapses (Gil-Loyzaga \& Pujol, 1988). Depending on the orientation of the cells and the angle of sectioning, these efferent synapses might appear like they are correlating with the IHC marker. This impression can be enhanced by the fact that imaging for the antibody screening was done with a normal epifluorescence microscope, meaning that the resolution was not sufficient to distinguish between very close synapses. Most of the time though, for synaptophysin, visual inspection was enough to distinguish between signals from efferent synapses and from ribbon synapses within the IHC (Figure $1 \mathrm{~A}$, last column). For the investigated proteins of interest, it was verified that the signal was indeed coming from within the IHC by super-resolution imaging at a later stage of the project. In a few cases, co-staining with synaptophysin could be used to clarify which signal originates from efferent synapses and which does not (see subsection 5.3.2). In the case of the POI Munc18 the correlation coefficient was not highly significantly different to the one from the GFP control ( $p=0.032$ ) and upon visual inspection the signal did not seem to be reliably originating from within the IHCs. In order to avoid including proteins with such unclear signal 
in further analyses, I excluded Munc18, from the list of target proteins for further investigation. In return, I included syntaxin 16 as target protein worth investigating further, since it has been reported to be expressed in IHCs and it has been detected already via immunolabeling using the same antibody (Revelo et al, 2014). Lastly, I decided to exclude the antibody detecting VAMP1/2/3, since the signal was most likely coming from the labeling of VAMP3, which could be detected by using another specific antibody, while antibodies detecting VAMP1 and VAMP2 exclusively did not show reliable signals and it has been reported that these proteins are not expressed in IHCs (Nouvian et al, 2011). In summary, I selected the following proteins for further analysis: Amphiphysin, AP180, Clathrin heavy chain (HC), CSP, Dynamin, Endophilin, Rab3, SCAMP1, SNAP29, Synaptogyrin, Synaptojanin, Synaptotagmin 2, Synaptotagmin 7, Syntaxin 12/13, Syntaxin 16, Syntaxin 6, VAMP3, VAMP4 and vATPase. 
A Otoferlin
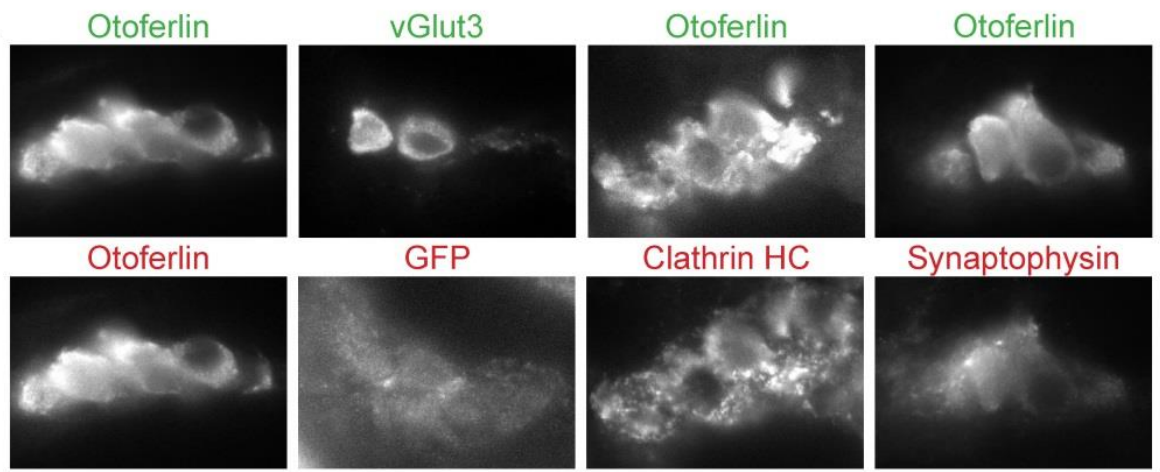

Clathrin $\mathrm{HC}$

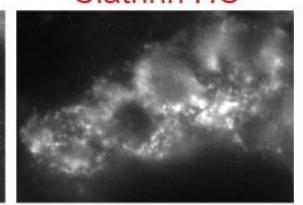

Synaptophysin
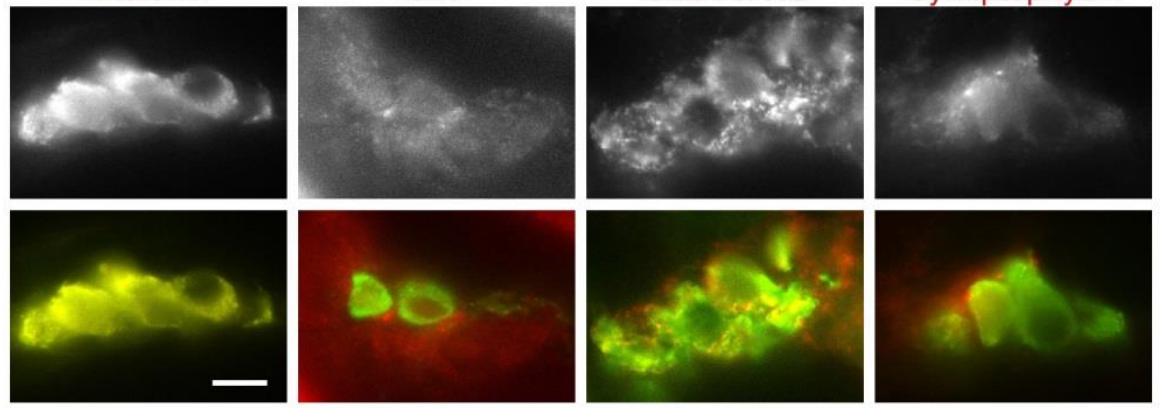

B
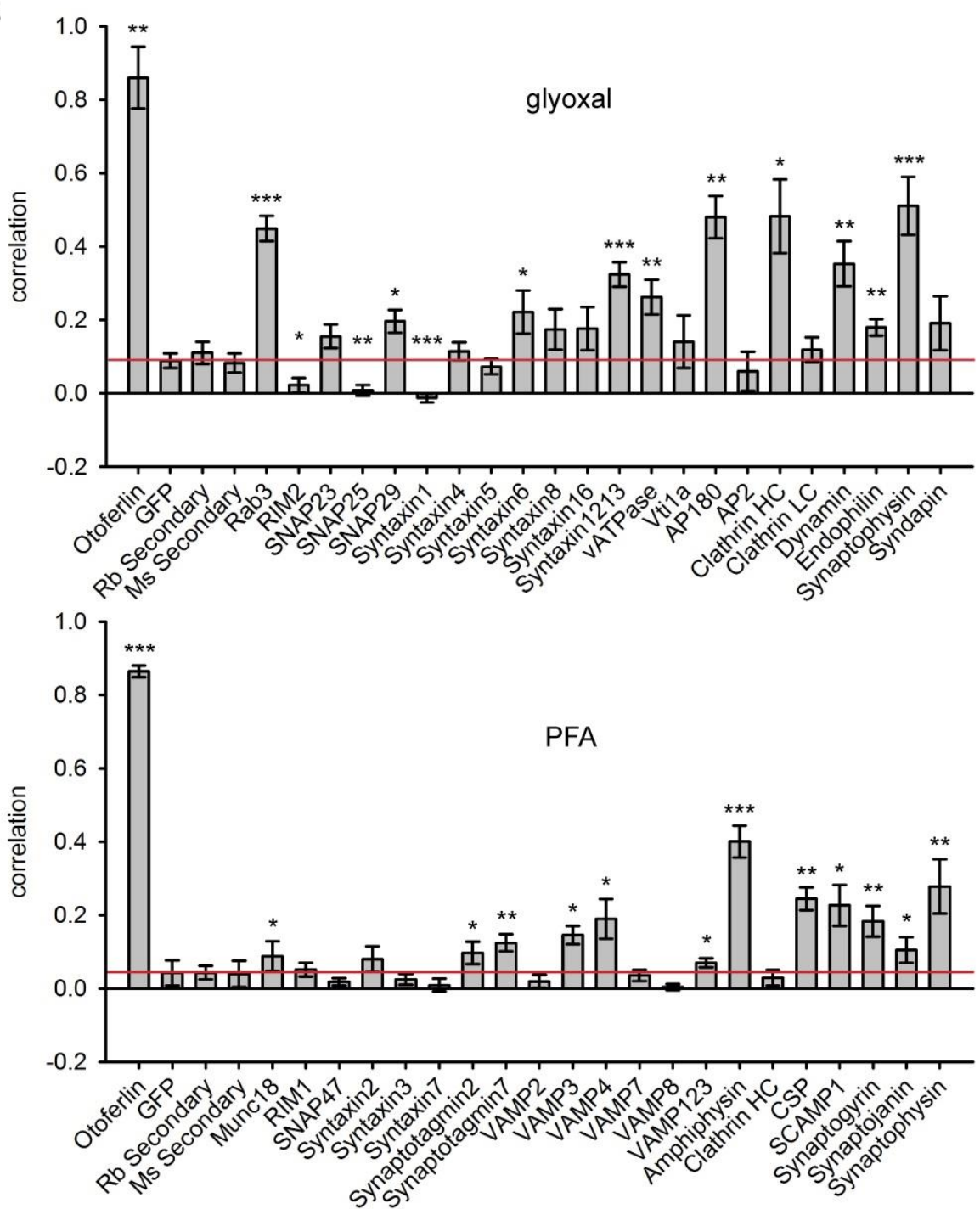

Figure 1 Screening for functioning antibodies against synaptic proteins in IHCs.

Evaluation of antibodies against synaptic proteins was done by correlation of the staining signal with an IHC specific staining (otoferlin or vGlut3) and comparison to a positive and negative control. A) Example images of such stainings used for correlation. The positive control staining (first column) consisted of labeling the IHC marker otoferlin with the same primary antibody and two differently 
labeled secondary antibodies. The negative control consisted of an anti-GFP staining in addition to the IHC marker vGlut3 (second column). The correlation of IHC marker and POI (shown here are clathrin $\mathrm{HC}$ and synaptophysin; third and last column) is compared with the correlation for positive and negative control stainings. B) Quantification of the correlations of various POls to the IHC marker. $\mathrm{Rb}$ secondary and Ms secondary are control stainings with secondary anti rabbit and anti mouse antibodies (without primary antibody) respectively. Some stainings were done on glyoxal fixed slices (upper graph) and some on PFA fixed slices (lower graph). Red lines mark the mean correlation resulting from anti-GFP stainings. Stars indicate significant differences to the correlation resulting from the negative control. $\mathrm{N}=6-32$ images from $3-6$ independent stainings, ${ }^{*} \mathrm{p}<0.05$, ${ }^{* *} p<0.01,{ }^{* *} \mathrm{p}<0.001$ (two-tailed Student's t-test for normally distributed data and Wilcoxon rank-sum test for not normally distributed data), scale bar $=10 \mu \mathrm{m}$.

In the following section I will give a brief overview of the POls selected for further investigation by super-resolution microscopy for the localization of these proteins and for copy number estimation. The target proteins can be divided into 3 major groups: exocytosis proteins or proteins associated with the synaptic vesicle membrane, endocytosis proteins and SNARE proteins that might function as alternatives to the commonly known synaptic SNAREs (SNAP25, syntaxin1 and VAMP2), which have been suggested to not be expressed in IHCs (Nouvian et al, 2011).

Exocytosis proteins/ synaptic vesicle membrane associated proteins:

Rab3 is a small GTPase, which is localized to the synaptic vesicle membrane in neurons (Südhof, 1995; Rizzoli, 2014). Four isoforms of the protein are known (rab3A - D) and seem to be somewhat redundant in their function (Schlüter et al, 2004). The isoform rab3A, which I focused on in this study, is thought to be involved in the regulation of late steps in $\mathrm{Ca}^{2+}$ mediated exocytosis (Geppert et al, 1997), possibly the priming of synaptic vesicles (Schlüter et al, 2006). In IHCs, rab3 is also associated with the synaptic vesicle membrane and thus has been used as synaptic vesicle marker (Revelo et al, 2014).

$\operatorname{CSP} \alpha$, or cysteine string protein, has also been found on the synaptic vesicle membrane in neurons (Takamori et al, 2006) and is, like rab3, involved in the late steps of synaptic vesicle exocytosis. It has been shown that CSP acts as a chaperone (Chamberlain \& Burgoyne, 1997) and is responsible for the stability of the SNARE protein SNAP25 and the formation of the synaptic SNARE complex (Sharma et al, 2011). IHCs have been shown to express CSP $\beta$ in addition to CSP $\alpha$ with at least partially redundant functions (Schmitz et al, 2006).

Synaptotagmin 2 and synaptotagmin 7 are both $\mathrm{Ca}^{2+}$ binding proteins, reported to be involved in regulating $\mathrm{Ca}^{2+}$ dependent exocytosis in neurons (Pang et al, 2006; Sugita et al, 2001). Synaptotagmin 2, an integral membrane protein of the synaptic vesicle, exhibits high similarity to synaptotagmin 1 , the highly abundant $\mathrm{Ca}^{2+}$ sensor protein of synaptic vesicles (Geppert et al, 1991; Takamori et al, 2006; Wilhelm et al, 2014). Synaptotagmin 7 is located on the plasma membrane and has been proposed to function as additional $\mathrm{Ca}^{2+}$ sensor complementary to vesicular synaptotagmins ( 1 and 2 (Sugita et al, 2001)). Synaptotagmin 2 and 7 have both been shown to be expressed in IHCs prior to the onset of hearing at P12 in mice (Beurg et al, 2010). 
The vacuolar proton pump, also called vATPase, is found in low numbers on the synaptic vesicle membrane of neurons as well (Takamori et al, 2006; Wilhelm et al, 2014). It is responsible for the acidification of the vesicle lumen to create a proton gradient and ultimately enable the filling of the synaptic vesicle with neurotransmitters (Südhof, 2004; Rizzoli, 2014). Not much is known about the presence of vATPases on synaptic vesicles of IHCS, but it has been detected in the presynaptic area of chicken cochleas via coimmunoprecipitation with anti-ribeye antibodies (Uthaiah \& Hudspeth, 2010).

Another protein associated with the synaptic vesicle membrane is Synaptogyrin (Südhof, 1995; Takamori et al, 2006). The exact function of this protein is unclear, but it has been shown to play a role in modulating the synaptic vesicle exo- and endocytosis cycle and biogenesis of synaptic vesicles (Stevens et al, 2012). Synaptogyrin was found in ribbon synapses of the mammalian retina (Von Kriegstein et al, 1999), but has not been reported in cochlear ribbon synapses so far.

Endocytosis proteins:

Synaptic vesicle exocytosis in conventional neurons is mostly compensated by clathrinmediated endocytosis. Clathrin light and heavy chain are the 2 major parts of the clathrin coat that surrounds pits and vesicles during recycling of the synaptic vesicles after exocytosis. Clathrin heavy and light chain assemble to triskelions to form the vesicle coat with the help of adapter proteins like AP2 and AP180 (Rizzoli, 2014; Haucke et al, 2011; Südhof, 1995; Cremona \& De Camilli, 1997). In IHCs, clathrin mediated endocytosis has been shown to at least contribute to the compensatory recycling mechanism, in addition to a faster endocytosis process, possibly bulk endocytosis (Neef et al, 2014; for a more detailed discussion see section 5.4).

Like mentioned before, AP180 is one of the adaptor proteins linking the clathrin coat to receptors in the synaptic vesicle membrane that have to be retrieved from the plasma membrane (Prasad \& Lippoldt, 1988; Hao et al, 1999; Koo et al, 2011). Presence of AP180 at IHC ribbon synapses is not clear yet, but a similar adaptor protein, AP2, has been shown to play an important role in synaptic vesicle recycling in IHCs (Pangršič \& Vogl, 2018; Jung et al, 2015).

A group of proteins interact with each other and regulate the process of clathrin mediated endocytosis. Amongst these are the SH3-domain containing proteins amphiphysin and endophilin and the phosphoinositide phosphatatse synaptojanin (Slepnev \& De Camilli, 2000; Haucke et al, 2011; Rizzoli, 2014). Amphiphysin and endophilin also contain BARdomains, through which they are recruited to and generate membrane curvatures (Mim \& Unger, 2012; Bai et al, 2010). Via the SH3 domains, both proteins recruit and bind to synaptojanin, which regulates clathrin coat assembly by hydrolyzing phosphatidylinositol $(4,5)$ bisphosphate $\left(\mathrm{PIP}_{2}\right)$ (Slepnev \& De Camilli, 2000). Amphiphysin in addition binds to clathrin and the GTPase dynamin (Slepnev \& De Camilli, 2000; Haucke et al, 2011). Synaptojanin and endophilin have been detected in cochlea preparations by immunofluorescent labeling and immunoblot experiments in the past (Uthaiah \& Hudspeth, 2010) and recently the presence of endophilin in IHC ribbon synapses has been confirmed 
(Kroll et al, 2019). The expression of amphiphysin in IHC ribbon synapses has been shown as well (Neef et al, 2014).

Another key protein in synaptic vesicle endocytosis is the previously mentioned GTPase dynamin, which is recruited by amphiphysin and endophilin (Slepnev \& De Camilli, 2000). It is thought that dynamin helps in fission of the coated vesicle from the plasmamembrane through its GTPase activity (Hinshaw, 2000; Faelber et al, 2012). At the ribbon synapse of the auditory hair cells, dynamin inhibitors have been found to reduce synaptic vesicle endocytosis, indicating that dynamin at least partially contributes to synaptic vesicle recycling in IHCs (Neef et al, 2014).

An ubiquitously expressed transmembrane protein is the secretory carrier membrane protein (SCAMP), which functions in membrane trafficking in general (Südhof, 1995). SCAMP1 is also found on synaptic vesicles (Takamori et al, 2006; Wilhelm et al, 2014) and interacts with proteins that are involved in the budding of vesicles from the membrane (Fernández-Chacón et al, 2000). SCAMP1 has been reported once to be present in cochlea hair cells via immunoblot and MS experiments (Uthaiah \& Hudspeth, 2010).

SNARE proteins:

The SNARE proteins syntaxin 6, syntaxin 12/13 (orthologes of the same gene, UniProt Id: G3V7P1) and syntaxin 16 are all known to function in trans-golgi and endosomal transport (Bock et al, 1997; Simonsen et al, 1999; Tang et al, 1998; Prekeris et al, 1998; Advani et al, 1998). Via interaction with other SNARE proteins, like VAMP3 and VAMP4, they mediate vesicle fusion to endosomes and the golgi network (Advani et al, 1998; Steegmaier et al, 1999; Mallard et al, 2002). In contrast to the highly debated presence of conventional synaptic SNARE proteins syntaxin 1, VAMP2 and SNAP25 (Safieddine et al, 2012), syntaxin 6 and syntaxin 16 have been reported to be detectable by immunostaining in IHCs (Revelo et al, 2014). While the presence of VAMP1 - 3 is still highly debated in the case of hair cell ribbon synapses (Safieddine \& Wenthold, 1999; Wenthold et al, 2002; Uthaiah \& Hudspeth, 2010; Nouvian et al, 2011), the expression of syntaxin $12 / 13$ and VAMP4 have not been investigated much.

SNAP29, another SNARE protein, which is similar to the synaptic SNARE SNAP25, is also located to the golgi network and interacts with syntaxin 6 (Wong et al, 1999), but has also been reported to localize ubiquitously to intracellular membranes (Steegmaier et al, 1998). Additionally, SNAP29 has been associated with autophagy (Itakura et al, 2012; Morelli et al, 2014). Whether SNAP29 is expressed in IHCs is not known.

\subsubsection{Super-resolution imaging of synaptic proteins for the precise localization within the ribbon synapse}

After having selected target proteins known to be involved in the synaptic vesicle recycling process and the appropriate antibodies to detect them, I proceeded with the staining and imaging of those target proteins using super-resolution microscopy. For STED imaging, I stained cryosections of the Organ of Corti for the POI and additionally for the IHC marker vGlut3 and the ribbon synapse marker CtBP2 or ribeye, which both label the ribbon. vGlut3 
staining was only used to identify IHCs and thus imaging in confocal resolution was sufficient. The stainings of the synaptic ribbon and the protein of interest were imaged in STED mode, using the laser line providing better resolution (640nm) for the POI (Figure 2A). In order to provide an average distribution of the protein of interest within the ribbon synapse of the hair cell, I stained and imaged at least 4 cryosections per POI independently, each containing multiple ribbon synapses. The images of each synapse were aligned and rotated according to a model (Figure 2B), so the orientation of each synapse was the same and the staining signal could be averaged over every synapse of one cryosection (Figure $2 \mathrm{C}$ ) and finally over all synapses from all cryosections (Figure 2D). The resulting average distribution maps were generated for all target proteins (Figure 3).

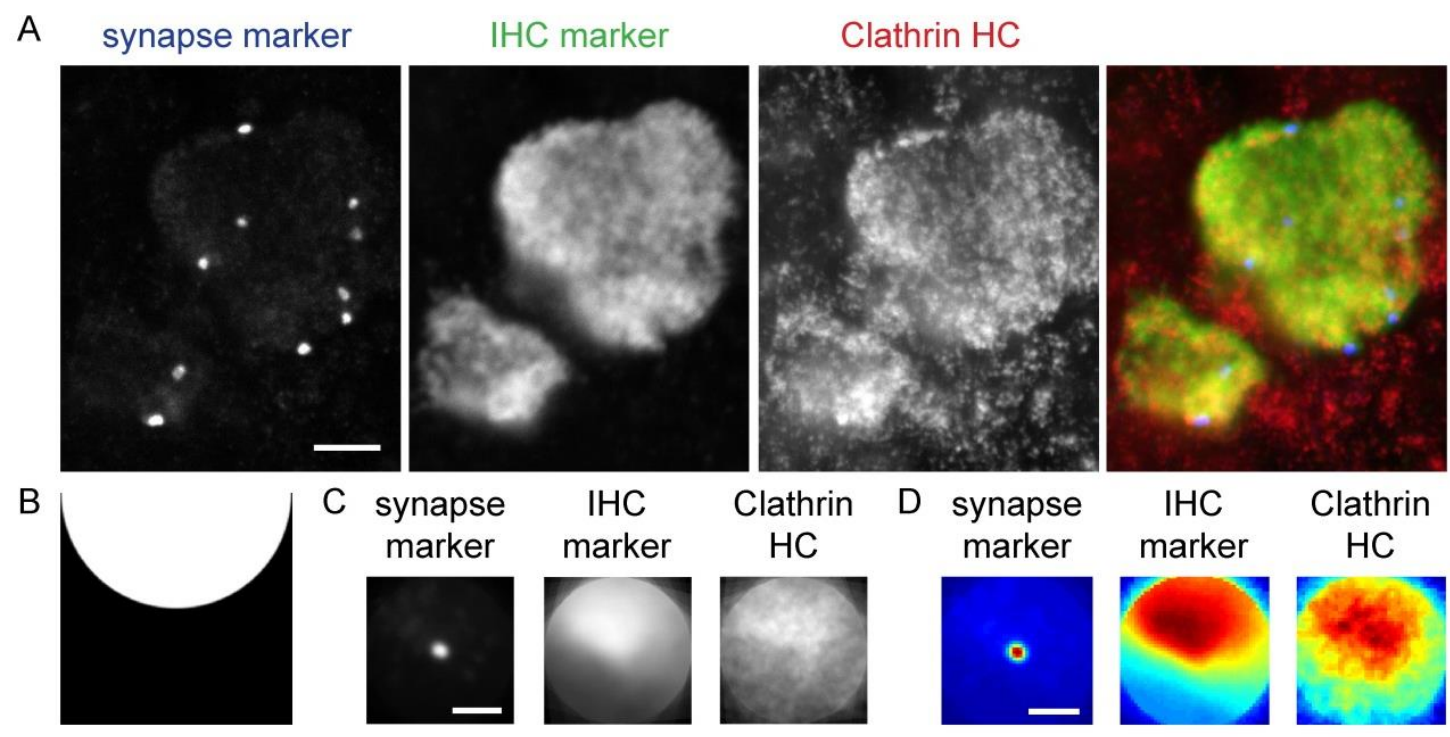

Figure 2 STED imaging for determining the spatial organization of synaptic proteins in the IHC ribbon synapse.

A) Example images of IHC ribbon synapses stained for the protein of interest clathrin (red). Samples were always stained for a ribbon synapse marker (ribeye or CtBP2; blue) and an IHC marker (vGlut3; green). The IHC marker was imaged in confocal mode, in order to locate the cells. Synapse marker as well as the POI were imaged in STED mode for precise localization of the ribbon and the protein of interest in relation to the ribbon. Scale bar $=2 \mu \mathrm{m}$. B) For the analysis of the spatial orientation of each POI, images of each ribbon synapse area were oriented according to a superficial model (B). This way all synapses were centered with the IHC cytosol (labeled by the IHC marker) facing the upper half of the image (white half circle in the model). C) Staining signals of each marker could be averaged over all synapses from one sample. D) Averaging signals over all samples resulted in average distribution maps. The color map indicates the intensity of the signal (from blue - low to red - high). Scale bar in C and D $1 \mu \mathrm{m}$.

From the average distribution maps, one can see that some proteins (amphiphysin, CSP and VAMP4) seem to have a very localized distribution with a few hotspots within the image area (Figure 3). Most proteins though seem to be distributed rather ubiquitously within the area centered around the ribbon. A few proteins show a distribution, which seems almost random within the whole area of analysis, not even matching the IHC cytosol labeling (SCAMP1, synaptogyrin, synaptotagmin 2, synaptotagmin 7, VAMP3, synaptojanin). Whether those signals originate from true protein distributions remains to be carefully discussed. 


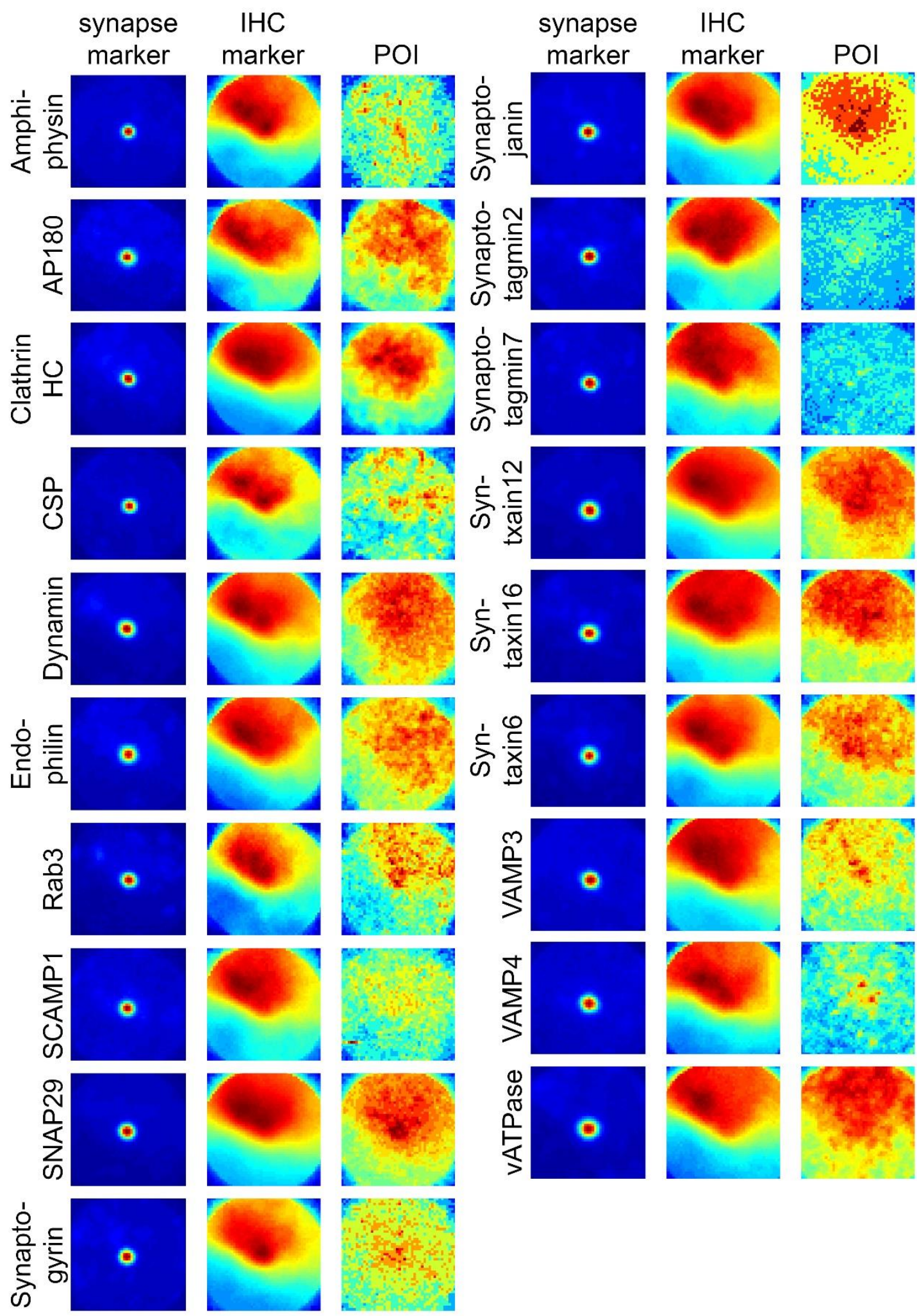

Figure 3 Average distribution maps of each POI in relation to the ribbon synapse marker and IHC marker.

Staining signals of the ribbon marker, the IHC marker and the protein of interest were averaged over all detected ribbon synapses, resulting from the imaging of $4-8 \mathrm{IHC}$ samples (cryosections) per POI. The color map indicates the intensity of the signal (blue - low; red - high) and is not scaled equally between different POI samples. Image dimensions are $3 \mu \mathrm{m} \times 3 \mu \mathrm{m}$. Some proteins of interest seem 
to be very localized (e.g. Amphiphysin, CSP or VAMP4), whereas most of the investigated proteins seem to be rather dispersed within the boundaries of the IHC marker.

During the imaging process I had the impression that for some proteins (CSP, endophilin and vATPase), a substantial part of the signal was localized outside the IHC, but close to the synaptic ribbons. These signals might originate from the proteins expressed in efferent synapses. In order to confirm this impression, I repeated the stainings of those POls with a co-staining of synaptophysin, which is known to be expressed in synapses of efferent neurons (Gil-Loyzaga \& Pujol, 1988; Figure 4). Strong co-localization of synaptophysin with major parts of the signal from CSP, endophilin and VATPase stainings were detected. This confirms that the strong labeling signal outside of the IHCs originate from efferent synapses. Nevertheless, less bright signal of those three POIs was also detected within the area of IHC labeling, which indicates that these proteins are also expressed in IHCS, although to a lower amount. This assumption is supported by previous studies, detecting these proteins in IHCs (Uthaiah \& Hudspeth, 2010; Eybalin et al, 2002; Hickox et al, 2017; Scheffer et al, 2015; Liu et al, 2014b; Kroll et al, 2019), as discussed in section 6.3. 

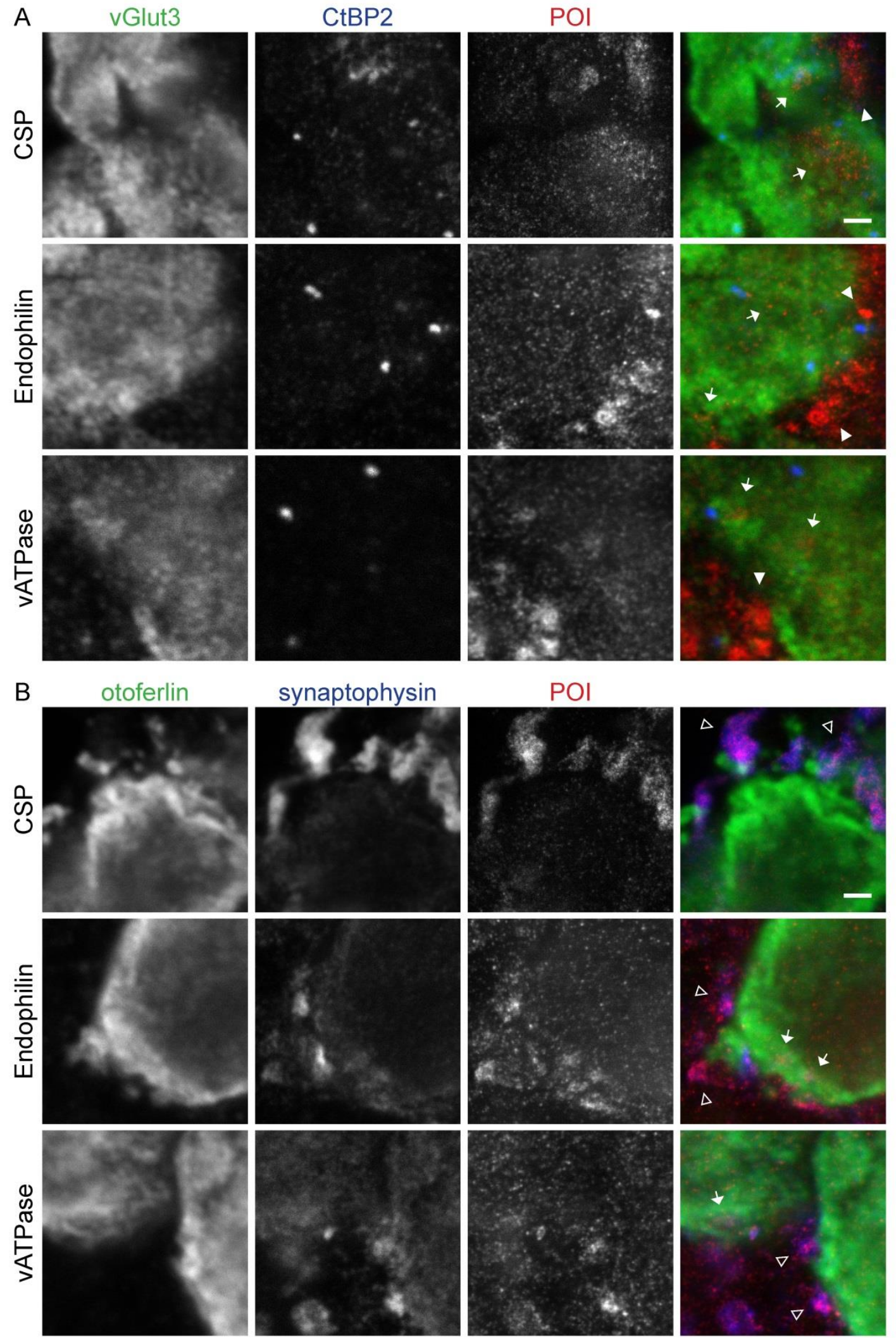

Figure 4 Co-immunostaining of synaptophysin and the POIs CSP, endophilin and vATPase.

A) Example images of the stainings used for protein localization. Bright labeling of the proteins of interest CSP, endophilin and VATPase (red) were found outside of the IHC marker area (green), but in the vicinity of ribbon synapses (arrowhead). B) Co-staining of the POls with synaptophysin as marker for efferent synapses. Strong co-localization of each target protein with synaptophysin can be observed (open arrowheads). Less bright signal of CSP, endophilin and VATPase stainings could also be found within the IHCs (arrows). Scale bar $=1 \mu \mathrm{m}$. 


\subsubsection{Protein copy number estimation using a comparative imaging approach (CosiQuant)}

In order to get an estimate for the copy number of each investigated protein in the IHC ribbon synapse, I used the comparative imaging technique CosiQuant that was introduced in chapter 04. To recapitulate, this method is based on the comparison of signals between a sample of interest and synaptosome preparations that have been immunostained in parallel for the same protein of interest. I have stained cryosections of the Organ of Corti and synaptosomes (the same preparations as in chapter 04) for all 19 POls using the same protocol and imaged the samples in parallel, using a confocal microscope. Small adjustments had to be made in comparison to the protocol in chapter 04 . The synaptosome samples were still immunostained for the two synapse markers synaptophysin and bassoon in addition to the POI, but IHCs were stained for the IHC marker vGlut3 and the ribbon marker CtBP2 or ribeye instead (Figure 5 and 6). The staining procedure for the POI was kept identical between IHC samples and synaptosomes. The signal of the two markers were used for the automatic identification of synapses and the intensity of the POI label was measured in that area. For each $\mathrm{POI}$, the amount of non-specific background signal was assessed by control stainings, using only secondary antibodies, without the primary antibody. These control stainings were performed and imaged under the same conditions as the IHC samples and the synaptosome samples. Thus, mean intensity values for the POls could be corrected for background signal. To account for differently sized areas of measurement, influencing the comparison of copy numbers between the different samples, the mean signal intensity was calculated for the number of pixels measured. By doing so, the intensities could be compared between different areas measured in IHCs and synaptosomes. The intensity for each immunostained POI in IHCs was then expressed as fold over synaptosome signal (Figure $6 \mathrm{~B})$ and from this ratio and the known copy numbers of the POIs in synaptosomes, I estimated the protein copy numbers in the IHC synapse (Figure 6B).

Although multiple independent stainings were imaged and analyzed to account for variability in the staining and imaging procedure, the mean intensities of the stained POIs still exhibit substantial variation (Figure $6 \mathrm{~B}$ ). This variability might represent differential staining efficiency or real variation in protein expression between different synapses (see section 5.4. for further discussion on this). As for the estimated protein copy numbers, one can sort the proteins into different abundancy categories for a better overview. By far the most abundant protein seems to be the small GTPase rab3, which resides on the synaptic vesicles. An intermediate abundancy can be seen for the endocytosis proteins clathrin, AP180, dynamin and amphiphysin. The proteins VATPase, SCAMP1 and endophilin show an intermediate to low copy number. The rest of the proteins seem to be present in low copy numbers, amongst these are all tested SNARE proteins, synaptojanin and the exocytosis proteins synaptotagmin 2 , synaptotagmin 7, CSP and synaptogyrin. 

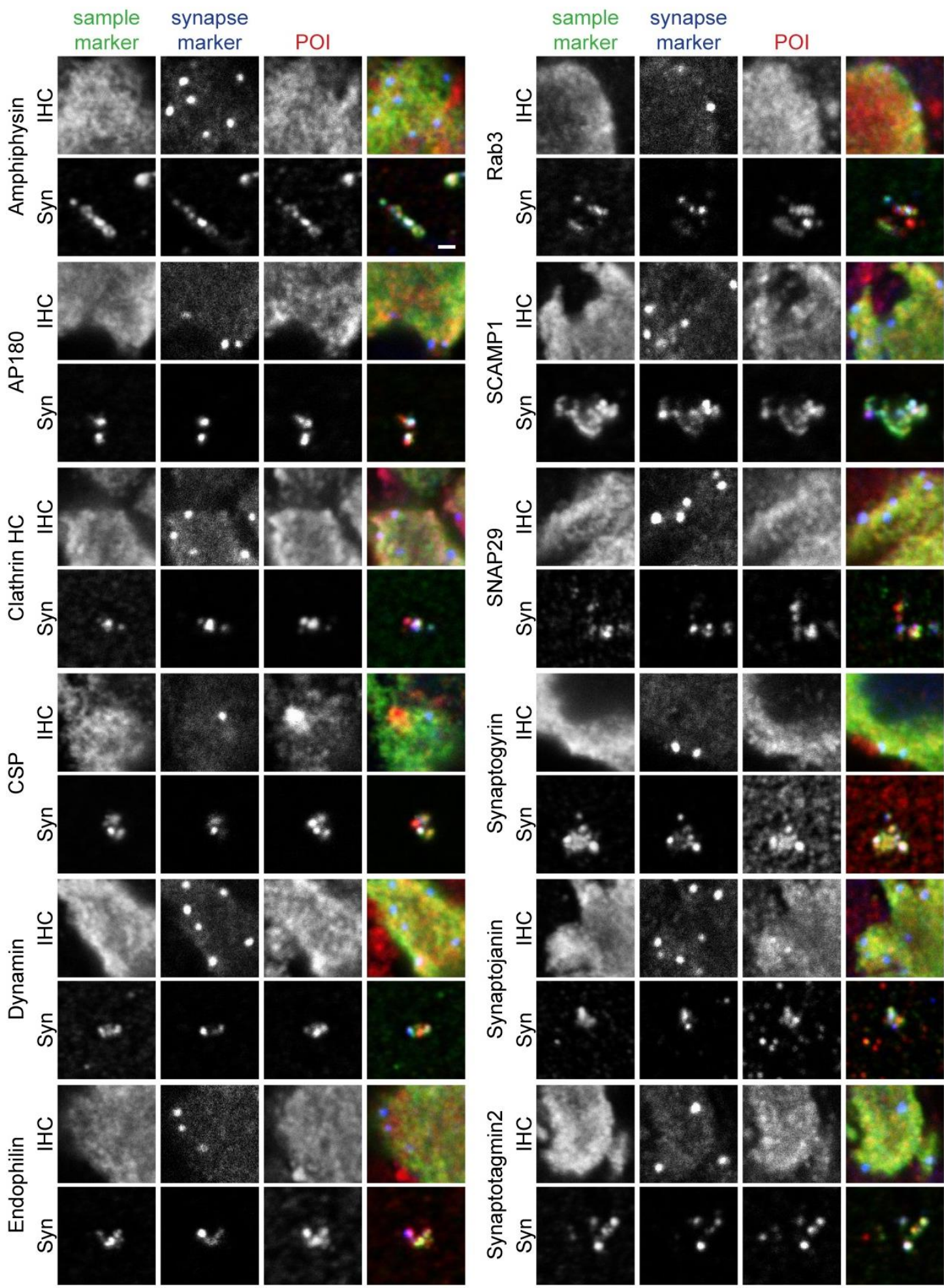

Figure 5 Protein copy number estimation by comparison of immunostaining signals.

Example images of IHC cryosections (IHC) and synaptosomes (Syn) stained and imaged in parallel for the same POI and different sample markers (synaptophysin for synaptosomes and vGlut3 for IHCs) and synapse markers (bassoon for synaptosomes and ribeye or CtBP2 for IHCs). Copy numbers for the POIs can be estimated from comparing signal intensities between biochemically characterized synaptosomes and IHC samples based on the CosiQuant method established previously in chapter 04. Images of synaptosomes and IHCs are scaled equally for the same POI stainings, images are not corrected for background signal here. Scale bar $=1 \mu \mathrm{m}$. 


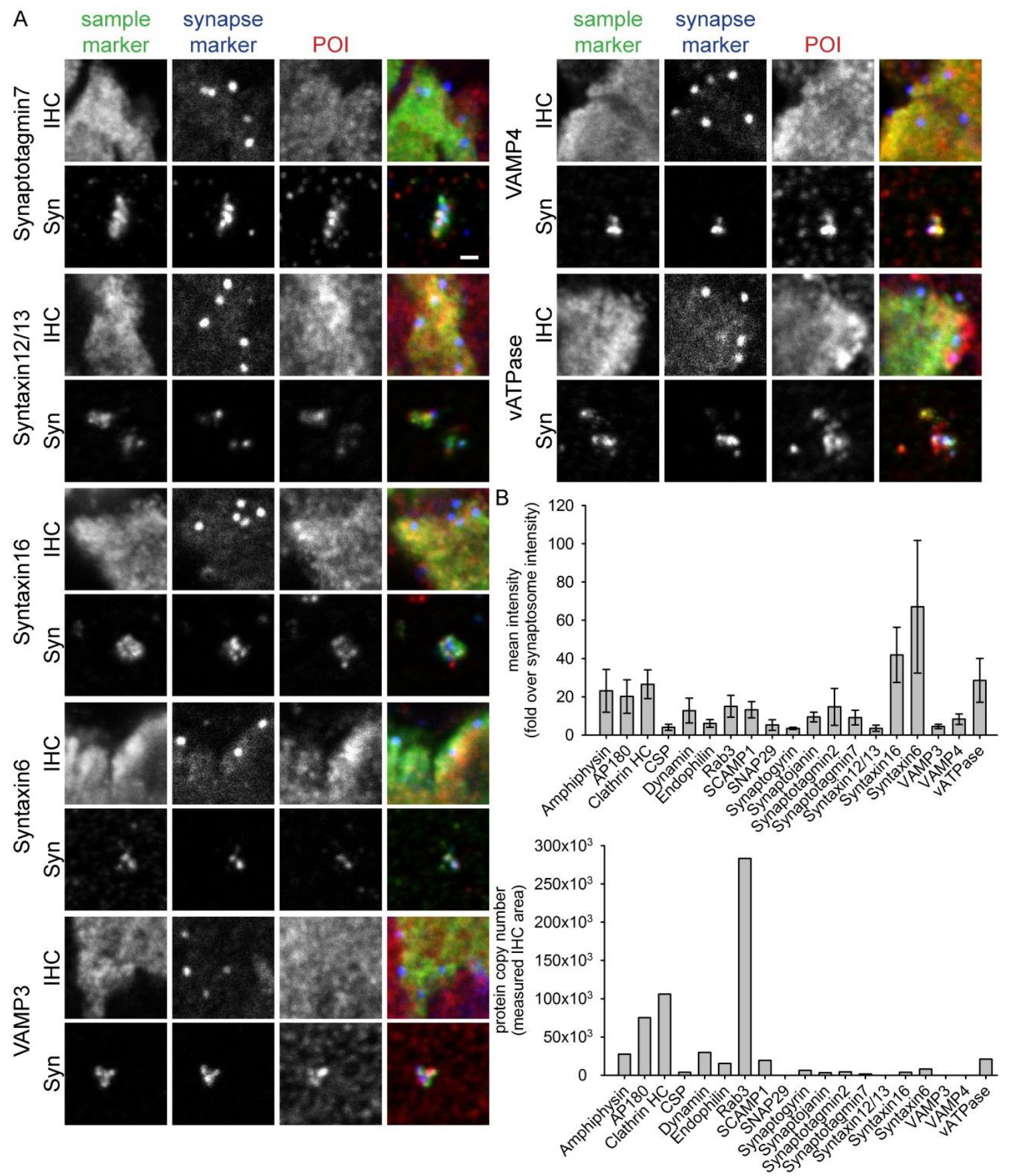

Figure 6 Protein copy number estimation by comparison of immunostaining signals (continued).

A) Example images of IHC cryosections (IHC) and synaptosomes (Syn) stained and imaged in parallel for the same POI and different sample markers (synaptophysin for synaptosomes and vGlut3 for IHCs) and synapse markers (bassoon for synaptosomes and ribeye or CtBP2 for IHCs). Images of synaptosomes and IHCs are scaled equally for the same POI stainings, images are not corrected for background signal here. Scale bar $=1 \mu \mathrm{m}$. B) Copy numbers for the POls (lower panel) can be estimated from comparing mean signal intensities of the POI (upper panel) between biochemically characterized synaptosomes and IHC samples based on the CosiQuant method established previously in chapter 04. Mean intensities of the POI in IHCs are background substracted signals, calculated for the measured area and expressed as fold over synaptosome signal. Copy numbers for VAMP3 are currently under evaluation by dot plot analysis, since the copy numbers in Wilhelm et al, 2014 were obtained using a different antibody. Error bars represent the SEM. $\mathrm{N}=3-7$ independent stainings containing multiple images. 


\subsubsection{Incorporation of protein copy numbers and spatial organization into a 3-dimensional model}

After having determined the localization and estimated the copy numbers of the selected synaptic proteins, I aimed to combine this data in a 3-dimensional model of the hair cell ribbon synapse. Therefore, I reconstructed a ribbon synapse from electron microscopy images (Figure 7) taken from serial sections of a wildtype mouse cochlea (P12). This electron microscopy data was kindly provided by Prof. Dr. Carolin Wichmann (University of Göttingen Medical Center, Göttingen, Germany). Serial sections of $60 \mathrm{~nm}$ thickness contained the complete structure of the ribbon and adjacent synaptic vesicles (Figure 7A). Manual tracing of structures and organelles like the plasma membrane, active zone, synaptic ribbon, synaptic vesicles and vacuoles resulted in a 3-dimensional model of the ribbon synapse (Figure 7B), which can be used as a basis for further modelling of the proteins within that synapse area.
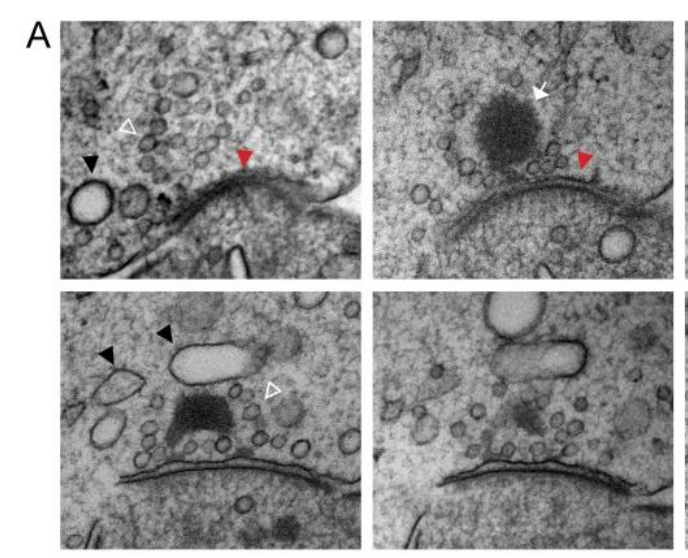
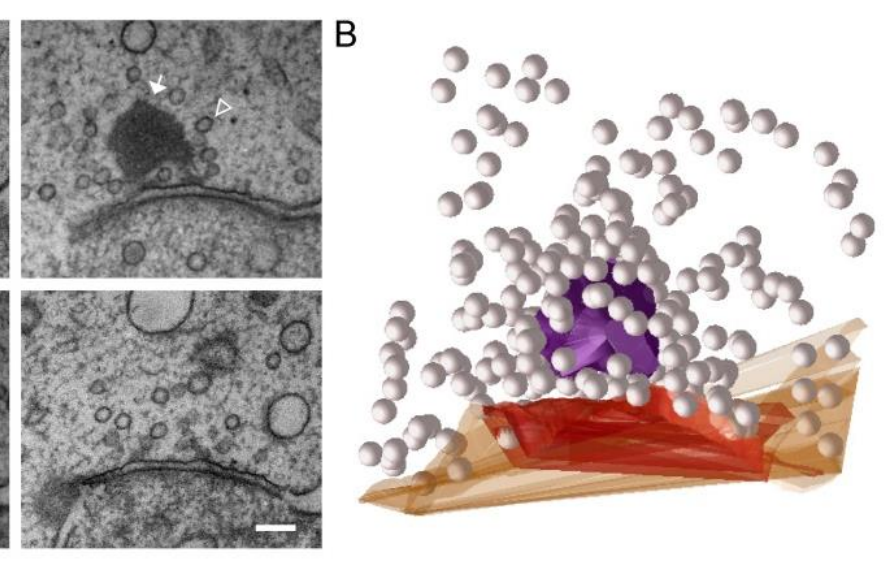

Figure 7 3-dimensional reconstruction of an IHC ribbon synapse.

A) Electron microscopy images of serial sections through an IHC ribbon synapse served as a basis for the 3-dimensional reconstruction of such a ribbon synapse. A few example sections are shown here, containing the plasma membrane with the active zone (red arrowhead), the ribbon (white arrow), synaptic vesicles (open arrowhead) and vacuoles (black arrowheads). Scale bar $=100 \mathrm{~nm}$. B) 3dimensional model of the ribbon synapse, consisting of the plasma membrane (brown) with the active zone (red), the ribbon (purple) and synaptic vesicles (grey). Vacuoles were traced and reconstructed as well, but are not shown here for better visibility.

In order to model the previously investigated proteins in correct abundancies, the estimated protein copy numbers needed to be calculated according to the reconstructed space of the ribbon synapse (model area in Table 2). Protein copy numbers were calculated from the previously estimated numbers within the specific imaging areas (measured area in Table 2), so they match the reconstructed area taken from the EM data. 
Table 2 Mean intensities and estimated protein copy numbers for synaptic proteins in IHCS Mean intensities are expressed as fold over synaptosome intensities; SEM = standard error of the mean. Measured area refers to the image area, on which the copy number estimation was based. Model area refers to the 3-dimensional model area based on electron microscopy data.

\begin{tabular}{|c|c|c|c|c|}
\hline POI & mean intensity & SEM & $\begin{array}{c}\text { protein copy number } \\
\text { (measured area) }\end{array}$ & $\begin{array}{c}\text { protein copy number } \\
\text { (model area) }\end{array}$ \\
\hline \hline Amphiphysin & 23.0777 & 11.1836 & 27559.3893 & 10218.7246 \\
\hline AP180 & 20.1603 & 8.7207 & 75327.1258 & 27930.4866 \\
\hline Clathrin & 26.5421 & 7.4984 & 106168.457 & 39366.12 \\
\hline CSP & 4.0508 & 1.5701 & 3812.5633 & 1413.6574 \\
\hline Dynamin & 12.7938 & 6.4514 & 29763.5739 & 11036.0124 \\
\hline Endophilin & 6.0927 & 2.0502 & 15380.5129 & 5702.9284 \\
\hline Rab3 & 15.028 & 5.6671 & 283226.404 & 105017.299 \\
\hline SCAMP1 & 13.2337 & 4.2182 & 19314.5435 & 7161.6246 \\
\hline SNAP29 & 5.2442 & 2.7598 & 406.2708 & 150.6408 \\
\hline Synaptogyrin & 3.4685 & 0.599 & 6433.448 & 2385.4532 \\
\hline Synaptojanin & 9.4347 & 2.553 & 3443.6777 & 1276.8786 \\
\hline Synaptotagmin2 & 14.7053 & 9.6276 & 4371.5767 & 1620.9336 \\
\hline Synaptotagmin7 & 9.1195 & 3.8221 & 1665.5763 & 617.5778 \\
\hline Syntaxin1213 & 3.5105 & 16,594 & 554.0609 & 205.4398 \\
\hline Syntaxin16 & 41.877 & 14.3859 & 3822.1174 & 1417.2 \\
\hline Syntaxin6 & 67.0355 & 34.6118 & 8156.2093 & 3024.2346 \\
\hline VAMP3 & 4.518 & 1.1203 & $*$ & $*$ \\
\hline VAMP4 & 8.267 & 2.7127 & 831.5775 & 308.34 \\
\hline vATPase & 28.5859 & 11.4616 & 21221.3146 & 7868.6348 \\
\hline
\end{tabular}

*copy numbers are currently being evaluated by dot plot analysis, since the copy numbers in Wilhelm et al, 2014 were obtained using a different antibody

To get a better graphical overview of the data obtained from this study, some preliminary modelling of the proteins within the reconstructed synapse was done. For this, the proteins were placed in a 3-dimensional coordinate system according to their estimated abundancy and localization determined by STED microscopy (Figure 8 and 9). The proteins are represented as simple green crosses until more advanced modelling can be done in cooperation with Burkhard Rammner (University of Göttingen Medical Center, Göttingen, Germany), who already modelled the presynaptic bouton in Wilhelm et al, 2014. For now, knowledge of proteins being associated to certain organelles (like the synaptic vesicle) has been neglected for simplicity purposes. Since the proteins I investigated in this project were based on the available protein numbers from the Wilhelm et al, 2014 study, the basic models of those proteins already exist and only need to be adjusted in number and location according to my data and the literature for a more advanced model of the ribbon synapse. 

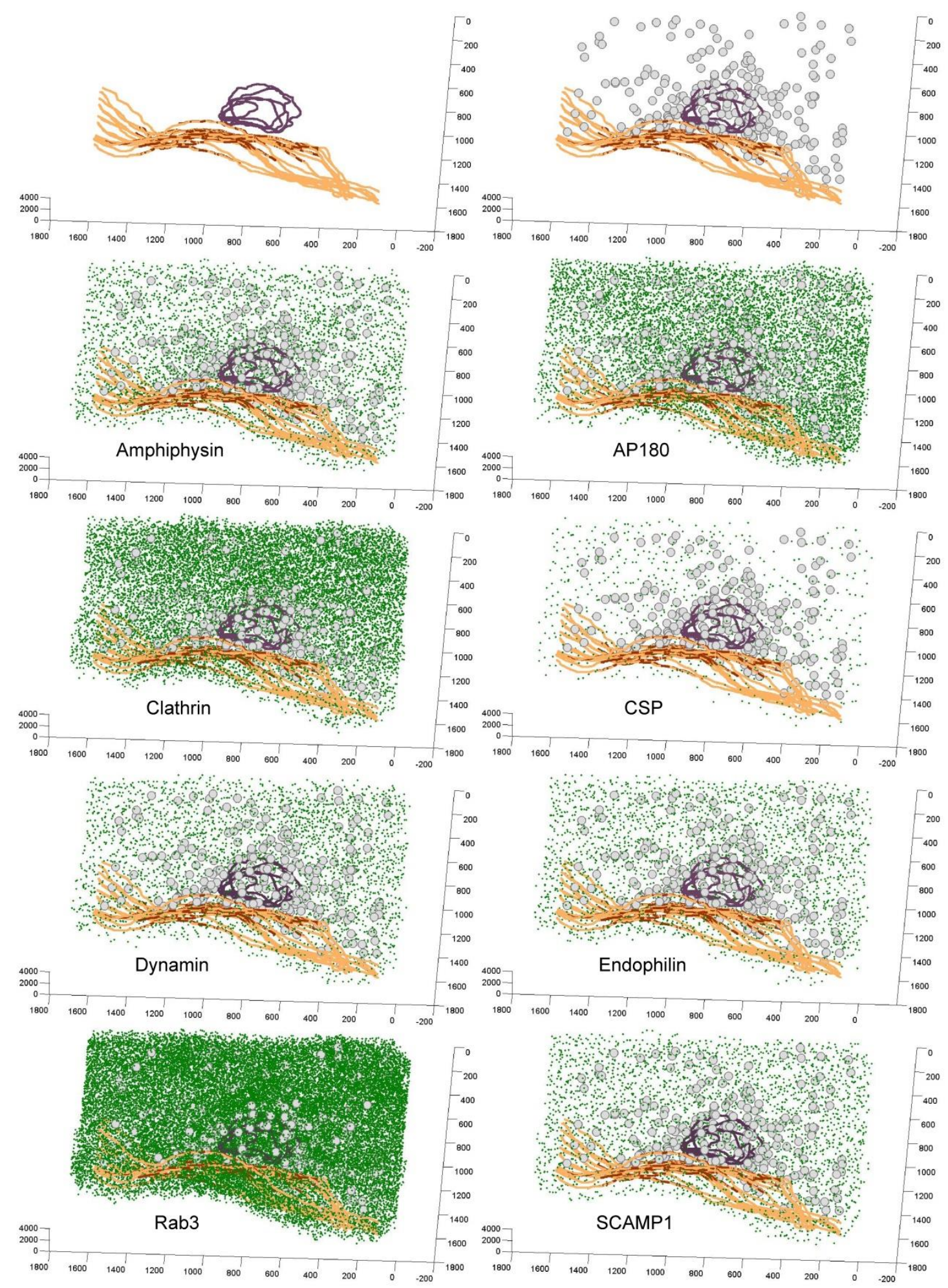

Figure 8 Preliminary modelling of the 3-dimensional synaptic space of the IHC ribbon synapse, containing the investigated proteins.

Tracings of the plasma membrane (brown), active zone (red), synaptic ribbon (purple) and synaptic vesicles (grey) per EM section are represented in a 3-dimensinal coordinate system. Each investigated POI (green) was placed in that synaptic space according to the copy number estimation and super resolution localization. For display purpose, the first 2 panels show an 'empty synapse', containing only the membrane, active zone, ribbon and vesicles. Thereafter, each POI is shown separately in that model of the ribbon synapse. 

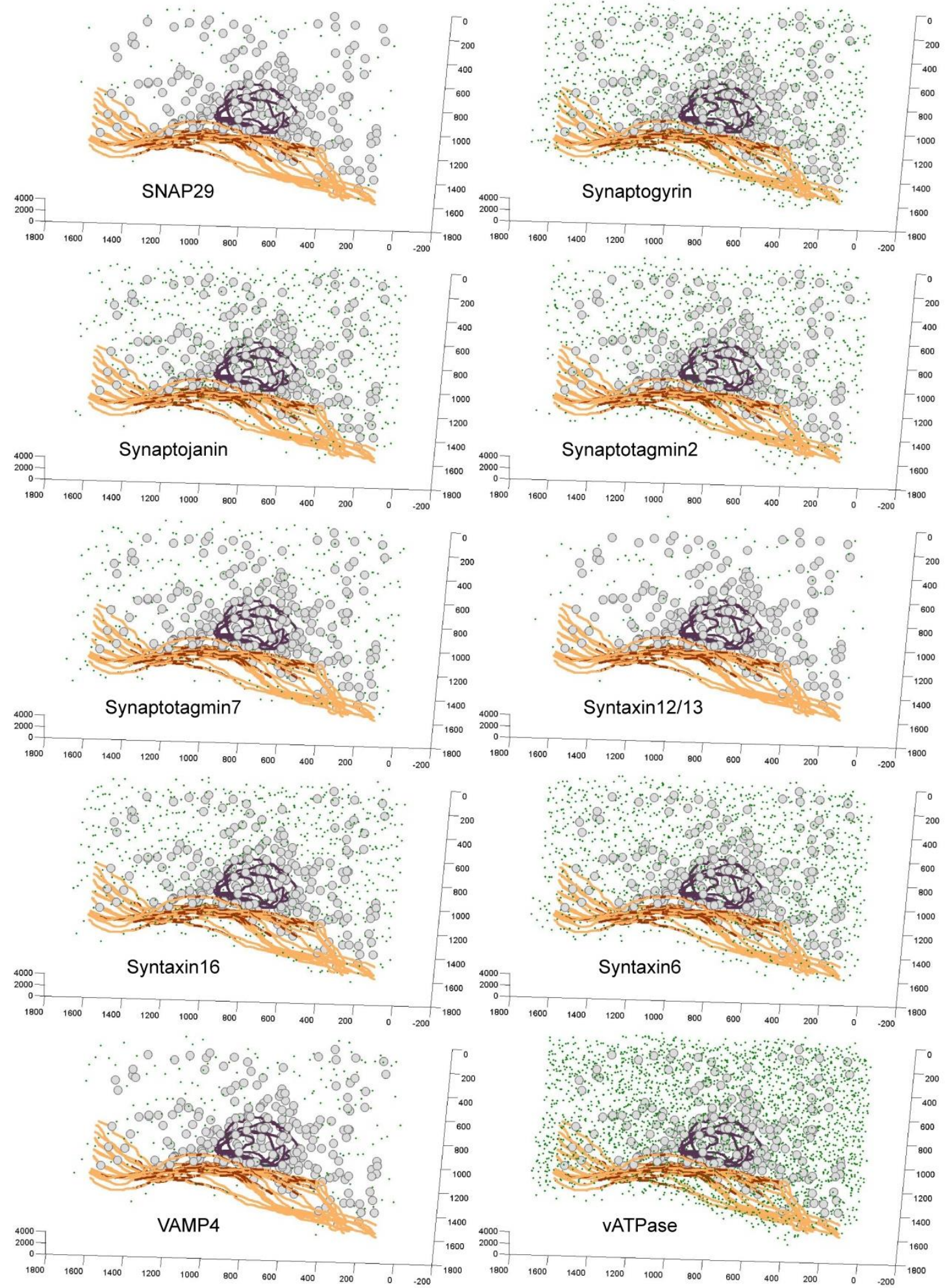

Figure 9 Preliminary modelling of the 3-dimensional synaptic space of the IHC ribbon synapse, containing the investigated proteins (continued).

Tracings of the plasma membrane (brown), active zone (red), synaptic ribbon (purple) and synaptic vesicles (grey) per EM section are represented in a 3-dimensinal coordinate system. Each investigated POI (green) was placed in that synaptic space according to the copy number estimation and super resolution localization. For display purpose, the first 2 panels show an 'empty synapse', 
containing only the membrane, active zone, ribbon and vesicles. Thereafter, each POI is shown separately in that model of the ribbon synapse.

\subsection{Discussion}

Due to its ability of very precise temporal stimulus-transmission coupling and the same time maintenance of sustained transmission, the IHC ribbon synapse is an interesting subject for investigating the mechanism of synaptic vesicle recycling. Vesicle recycling needs to be very efficient in these synapses in order to ensure synaptic transmission over a long time of stimulation without substantial fatigue. This is why it has been tried to unravel the details of this process in the past using various techniques, from electrophysiology to mass spectrometry. Nevertheless, until now the exact protein composition of the synaptic vesicle recycling machinery is unclear. Since this protein composition seems to differ from conventional synapses (Gil-Loyzaga \& Pujol, 1988; Safieddine \& Wenthold, 1997; Mandell et al, 1990; Roux et al, 2006; Michalski et al, 2017; Wenthold et al, 2002), it is important to have a clear picture of the molecular organization of the ribbon synapse in IHCs, before investigating detailed functions and interactions of proteins by genetic alterations and biochemical assays. In this study, I intend to provide such an overview of the IHC ribbon synapse, containing detailed information about the spatial orientation and abundancy of synaptic proteins within the presynaptic area. I achieved to do so by investigating the presence of 19 synaptic proteins and their exact location in relation to the ribbon by superresolution STED imaging. Fluorescence microscopy-based localization of ribbon synapse proteins has neither been done in such detail nor for more than $1-2$ proteins per study before. Implementing an improved fixation method and combining it with a precise colocalization of synaptic proteins and the ribbon by 2-colour STED microscopy, enabled me to conduct such a detailed investigation. Furthermore, the application of CosiQuant, a method to estimate protein copy numbers in samples which are difficult to investigate by biochemical techniques, allowed me to assess the abundancy of 19 proteins in the IHC ribbon synapse.

It has to be noted that CosiQuant is a semi-quantitative method and only provides estimates for protein copy numbers. This is especially relevant for the 19 synaptic proteins investigated in this study, since immunostaining signals of these proteins showed substantial variability (see Figure 6 in subsection 5.3.3). It is difficult to determine the cause of this variation in staining signals. It might be due to varied labeling efficiencies of the target protein by the primary and secondary antibody. This could be caused by different accessibilities of the epitope and different penetration efficiencies of the antibodies, depending on how the Organ of Corti was dissected and sectioned. Although at least penetration should not be a major problem in a $10 \mu \mathrm{m}$ cryosection of the organ, since whole Organs of Corti can be stained using similar staining protocols. Increased variability in fixation quality between the samples or target proteins using the new fixation method (glyoxal) can also be neglected, since variation of the immunostaining signal seems to be independent of which fixative was used. Alteration in imaging parameters (due to e.g. fluctuations in laser intensities of the 
setup) could potentially also contribute to the variation in measured signal intensities. This can however be neglected here, since synaptosome samples and IHC samples were always imaged in parallel on the same day. The high variance of the immunostaining intensity of the investigated proteins might also represent real variability between ribbon synapses. Despite dissecting the Organ of Corti in a buffer without $\mathrm{Ca}^{2+}$, there were no additional measures to inhibit or stimulate the synapses. Thus, variation in protein labeling signal might be due to different amounts of protein at the ribbon depending on the activity status, similar to the principle of different amounts of postsynaptic receptors in synaptic plasticity mechanisms at neuronal synapses (Fernandes \& Carvalho, 2016; Pozo \& Goda, 2010). Furthermore, ribbon synapses have been described to show high heterogeneity in morphology depending on their location within the IHC (modiolar side vs. pillar side) and along the tonotopic axis (Michanski et al, 2019; Safieddine et al, 2012). For imaging experiments, I only took the apical turn of the Organ of Corti to minimize the variability between synapses from different tonotopic positions, but I was not able to distinguish between ribbon synapses from modiolar and pillar sides. Thus, the variation in signal intensity for a certain protein might reflect the variation of protein expression at different ribbon synapses, according to the morphological parameters of the synapse.

Regardless of what causes the variation in immunostaining intensity, the estimates for protein copy numbers based on this signal is still valuable information, providing an overview of protein abundance at the IHC ribbon synapse. Amounts of the investigated proteins relative to each other and in comparison to protein amounts in conventional synapses now provide a starting point for evaluating the efficiency of the synaptic vesicle recycling process. It is possible to determine rate-limiting factors for this process, depending on the presence and need for a certain protein at the ribbon synapse (for detailed discussion see section 06). Additionally, this study presents further validation for the expression of specific proteins at the ribbon synapse. Most of the 19 investigated proteins have already been reported to be expressed in the IHC ribbon synapse, albeit some of them only once or twice in studies, which implement techniques and protocols not fully suited for the investigation of IHCS (e.g. the detection of synaptic vesicle proteins after immunoprecipitation of the ribbon, which only co-purifies a small amount of vesicles (Kantardzhieva et al, 2012) or the use of MS analyses in general). In these cases, my work can provide further evidence for protein expression by modern super-resolution microscopy (further discussion of the individual proteins studied in section 06).

In conclusion, having determined the precise location of 19 synaptic proteins within the ribbon synapse of the IHC and having estimated the copy numbers of those proteins, enabled me to present a preliminary model of the IHC ribbon synapse, which provides a semi-quantitative insight into the organization of the ribbon synapse and can further be used as a backbone for future findings to be added on. 


\section{$06 \mid$ General Discussion}

In this thesis I set out to study the molecular anatomy of the synaptic vesicle recycling process at the IHC ribbon synapse by implementing improved methods for the investigation of IHCs using imaging techniques. I managed to do this by first establishing an alternative fixation procedure to the commonly used PFA fixation. Additionally, I established an imaging-based method to estimate protein copy numbers in samples that are difficult to analyze in quantitative biochemical assays. I then combined those techniques in a detailed investigation of the molecular organization of the IHC ribbon synapse, focusing on the localization and determination of copy numbers of synaptic proteins in those synapses.

The use of glyoxal as an alternative fixative to PFA permitted me to overcome difficulties in immunostaining the Organ of Corti and IHCs, by assuring a more accurate fixation of the sample and consequently an improved quality of the immunolabeling of proteins. Chemical fixation, using PFA, has been reported to cause numerous fixation artifacts and even has been shown to fix some targets only incompletely (Schnell et al, 2012; Melan, 1994; Tanaka et al, 2010), which results in low quality immunostainings of such targets. Thus, improved fixation was necessary to be able to achieve the best possible staining quality of synaptic proteins in IHCS, which have been difficult to stain properly in the past by conventional staining protocols. Glyoxal, the smallest di-aldehyde, relies on the same chemical procedure of cross-linking for the fixation of proteins as PFA. However, we found it to act faster, conserve structures more accurately, and improve the quality of subsequent immunostainings, especially for super-resolution imaging. It was the optimal choice for improving the staining of some target proteins in IHCs, which have been difficult to visualize after PFA fixation.

For the analysis of protein abundancy at the IHC ribbon synapse, biochemical methods, such as mass spectrometry, have been used in previous studies (Uthaiah \& Hudspeth, 2010; Kantardzhieva et al, 2012; Hickox et al, 2017). MS is a powerful technique to accurately identify and quantify proteins, but has been difficult to apply to the IHC ribbon synapse without major contaminations from neighboring efferent synapses, due to the lack of sufficient purification (Uthaiah \& Hudspeth, 2010; Rutherford \& Pangršič, 2012). Furthermore, it only provides averages, since samples have to be pooled to get a sufficient amount for analyses. Thus, we established a method to estimate protein abundancy in IHC ribbon synapses, based on an imaging approach, making it possible to distinguish between individual synapses and thereby reducing contamination from efferent synapses. It relies on the comparison of immunostaining intensities of a protein of interest in biochemically well characterized synaptosomes and the samples of interest. We have termed this method CosiQuant, comparative synaptosome imaging for semi-quantitative copy numbers, and established a proof-of-principle using cultured hippocampal neurons. By immunostaining synaptosome preparations in parallel with hippocampal neurons (as sample of interest) for a specific target protein, we were able to compare staining intensities between the two samples and calculate copy numbers for the target protein, based on the known copy 
numbers in synaptosomes from previous analyses (Wilhelm et al, 2014). This technique allows for the estimation of protein copy numbers in samples that are difficult to quantify by other means, such as the IHC ribbon synapse.

In the third part of this thesis I combined the two methods for the investigation of proteins that are potentially involved in the synaptic vesicle recycling process at the IHC ribbon synapse. Using the improved fixation, and thus immunostaining protocol for IHC proteins and the CosiQuant technique, I was able to determine the precise location and estimate the copy numbers of 19 synaptic proteins of the IHC. I further visualized this data in a semiquantitative model of the protein organization in the ribbon synapse, using EM data as reference for basic morphological parameters.

I proceed below to discuss the different results from the three parts of my thesis, starting with the improvement of fixation by the use of glyoxal and its potential problems. Subsequently, I will discuss the advantages and limitations of CosiQuant and lastly, I will explain the findings of my study of synaptic proteins at the IHC ribbon synapse and discuss these in the context of the synaptic vesicle recycling process.

\subsection{Glyoxal, an alternative fixative to PFA}

In the first part of my thesis I was able to show that glyoxal can be used as an alternative fixative to PFA, improving the preservation of cell and organelle morphology, increasing the speed of fixation and probably crosslinking proteins more effectively. Moreover, I could show that the improved fixation by glyoxal results in better immunostaining quality for a variety of targets, especially visible in super-resolution microscopy. Together with 11 other laboratories around the world, I was able to prove that glyoxal fixation can be applied to a variety of different samples and target structures/proteins. Further we could show that glyoxal improves immunostaining signals for a substantial amount of these targets compared to PFA. Thus, glyoxal fixation brings a valuable opportunity to the scientific community to improve immunostainings. While glyoxal might not be able to enhance the fixation and thus immunostaining quality of every target structure, especially not for those targets whose fixation and immunostaining procedures have already been optimized to the fullest, it does provide an alternative that can be tested for targets that are known to be problematic in immunolabeling experiments. As mentioned above, glyoxal has been tested as an alternative to PFA fixation for immunofluorescence experiments in 11 laboratories, working on various different sample preparations and target proteins. Out of 56 targets tested in these studies, glyoxal was able to improve the immunostaining quality of 31 targets and provided an equal quality for 14 targets. Only 11 targets were immunostained with worse quality than after PFA fixation (Figure 10). Below I will briefly discuss the results from the different collaborating laboratories.

The laboratory of Edward Boyden compared the fixation and subsequent immunostaining of the nucleoporin complex protein NUP160 in cultured HeLa cells. Imaging via confocal microscopy showed that fixation with a glyoxal solution of $\mathrm{pH} 4$, resulted in a higher fluorescence intensity than after PFA fixation, although glyoxal pH 5 did not improve the staining intensity. These experiments illustrate nicely the dependence of glyoxal fixation on 
the $\mathrm{pH}$ of the solution. This has been reported before for glyoxal (Dapson, 2007), but does not seem to be relevant for PFA fixation, since PFA fixation of proteins in a solution of $\mathrm{pH} 4$ or 5 does not change fixation quality (Figure S7 from chapter 3).

The fixation and subsequent immunostaining of the proteins syntaxin 1, SNAP25 and LC3B by Rory Duncan's laboratory showed that glyoxal fixation is able to improve the immunostaining intensity for the plasma membrane SNARE proteins synatxin 1 and SNAP25, but not the autophagosomal membrane protein LC3B. Here, glyoxal was used in a solution of $\mathrm{pH}$ 5. Unfortunately, a solution of $\mathrm{pH} 4$ was not tested, which might have provided a different outcome for autophagosome markers.

A comparison of immunostaining quality after glyoxal and PFA fixation in super-resolution microscopy was provided by Stefan Hell's and Elisa D'Este's laboratory. Investigation of the fine structures of various cytoskeletal elements revealed that the effects of glyoxal fixation can be diverse. While glyoxal fixation did not seem to be as well suited as PFA for the staining of Ankyrin G, neurofilament stainings showed improved intensities. Even in comparison with a much stronger fixative, glutaraldehyde, glyoxal fixation was able to provide similarly well preserved structures, at least for some targets, like actin filaments in growth cones of neurons. Glutaraldehyde has been known to be an excellent fixative for cytoskeletal proteins, but does often reduce immunogenicity for other target proteins and therefore often does not permit co-staining (Farr \& Nakane, 1981). Glyoxal fixation seems to provide a similar immunostaining quality for cytoskeletal proteins, without reducing the quality of most co-stainings.

Marcel Lauterbach provided a test for glyoxal fixation in a rather unconventional sample, the sepia fin. $100 \mu \mathrm{m}$ thick cryosections of the sepia skin sample were immunostained for the neuropeptide FMRFamide. Confocal imaging showed major differences in the preservation of the sample, which was much better in glyoxal fixed tissue. This might be caused by the better penetration and faster overall fixation process achieved with the glyoxal solution. Thus, glyoxal fixation might especially be interesting for studies on thick samples, where fast penetration and fixation is needed in order to preserve structure.

Glyoxal fixation was also tested on very sensitive samples, like freshly dissected ventricular myocytes of mice (Stephan Lehnart laboratory). Fixation and immunostaining of calveolin-3 and ryanodine receptors not only showed that structures were preserved well by glyoxal, but also that the immunostaining intensity was increased for both targets after glyoxal fixation. This suggests that glyoxal can also be applied to sensitive samples, even though the low $\mathrm{pH}$ of the solution does not provide optimal physiological conditions.

In Tobias Moser's laboratory glyoxal and PFA fixation were compared in a similarly sensitive sample, the Organ of Corti. Whole organs were fixed and immunostained for 6 different proteins. The fluorescence intensity of 3 out of those 6 proteins was significantly increased after glyoxal fixation and none of the proteins was stained less well than after PFA fixation. These results indicate that glyoxal is suitable for the fixation of small whole organs. It has the potential to increase the immunostaining signal of some targets, while it keeps equal staining quality for those targets that are already well stained after PFA fixation. 
After the fixation of human neuroglioma cells, Tiago Outeiro's laboratory found that glyoxal could improve the staining quality of alpha-synuclein, but not endogenous vimentin. More interestingly, they also showed that glyoxal could be used for the fixation of overexpressed and fluorescently tagged vimentin in those cells. In comparison to PFA it even enhances the signal intensity of the tag.

Comparison of glyoxal and PFA fixation in Blanche Schwappach's and Perter Rehling's laboratories once more illustrate the diverse effects glyoxal fixation can have on different targets. While glyoxal fixation does not seem to be very suitable for most mitochondrial proteins ( 2 out of 5 proteins were stained better, but 3 were stained worse than after PFA fixation), it was able to improve the immunostainings of various other proteins, exhibiting diverse functions. Out of 18 fixed and immunostained proteins in cultured cells, 8 showed an increased fluorescence intensity after glyoxal fixation, 6 were equally well stained as after PFA fixation and only 4 proteins showed significantly decreased signal intensity. The effect of glyoxal fixation on subsequent immunostainings did not show any obvious correlations to the function or localization of the target protein. Interestingly, here the immunostaining of the autophagy marker LC3 was increased after glyoxal fixation, although it has been reported to not be affected positively by glyoxal fixation before (see results from Rory Duncan's laboratory). Both studies used different antibodies for the staining of the same protein, which indicates that the effect of glyoxal fixation might not only depend on the target protein, but also on the staining procedure and antibodies used.

The results from Ilaria Testa's laboratory, which tested glyoxal fixation on neurons and U2OS cells, illustrate another important issue of fixation: protein mislocalization. The immunostaining of the $\mathrm{Na}^{+} / \mathrm{K}^{+}$ATPase in neurons after PFA fixation shows the majority of the signal in the nucleus and cytoplasm of the cells, whereas in glyoxal fixed cells, most the signal can be seen in membranes. Since the $\mathrm{Na}^{+} / \mathrm{K}^{+}$ATPase is a transmembrane protein, it is obvious that the protein is mislocalized after PFA fixation. This has been shown before (Schnell et al, 2012) and is probably due to slow and incomplete fixation. Glyoxal seems to eliminate this issue at least for this protein. A similar, although not as severe, signal mislocalization was observed for an endoplasmatic reticulum marker in U2OS cells, while the mitochondrial protein TOM20 was stained with equal quality after both PFA and glyoxal fixation.

Finally, Bolek Zapiek tested glyoxal fixation of complex tissue samples from the mouse olfactory system. Perfusion with glyoxal and subsequent cryosectioning of the olfactory epithelium and olfactory bulb permitted the immunostaining of target proteins like neuropilin 2 or vGlut2, which could not be sufficiently stained after fixation with PFA. Moreover, fine structures, like cilia of the olfactory epithelium or dendritic structures in glomeruli of the olfactory bulb, are preserved better after glyoxal fixation than after PFA fixation. 


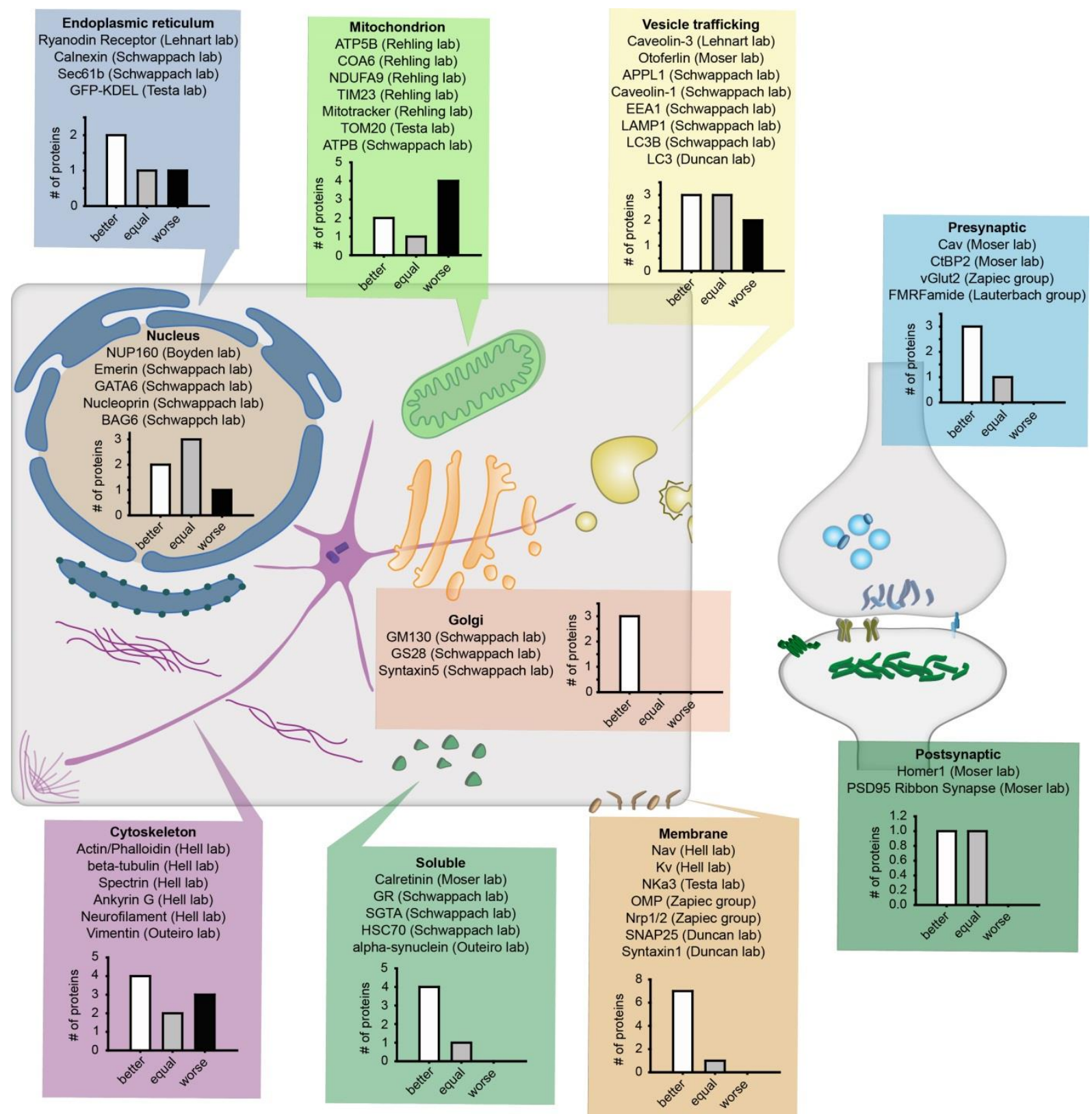

Figure 10 Summary of results from the comparison of glyoxal fixation to PFA fixation by 11 different laboratories.

Glyoxal fixation and subsequent immunostaining of various target proteins in different samples was tested and compared to PFA fixation. Out of 56 target proteins tested in the different collaborating laboratories, 31 were found to be immunostained better after glyoxal fixation than after PFA fixation. For 14 proteins glyoxal fixation led to an equal quality of immunostaining compared to PFA fixation and 11 proteins were immunostained worse after glyoxal fixation.

In summary, the results from all of these different studies indicate that glyoxal fixation can be applied to various different samples (ranging from relatively simple cell cultures to complex tissue sections and whole organs, as well as to very sensitive samples) and has the potential to help improve research based on immunofluorescence staining experiments.

Nevertheless, some issues might also arise with the usage of glyoxal as alternative to PFA fixation. The extensive testing of glyoxal fixation on a range of different sample preparations and different target proteins also illustrated that the effect glyoxal has on the fixation and immunostaining of a target varies, depending on the $\mathrm{pH}$ of the fixation solution, on the 
nature of the target protein and probably also on the antibodies used for detection. Since no obvious correlation exists between the nature of the target protein and the effect of glyoxal fixation on the immunostaining quality, it cannot be predicted, whether glyoxal fixation might improve the immunostaining of a certain protein in a certain sample. Thus, extensive testing of fixation and staining conditions, including different $\mathrm{pHs}$ and antibodies, needs to be done in order to determine whether glyoxal fixation can improve the immunostaining quality. This is impractical for targets, where PFA fixation and optimized staining procedures already provide good, or at least acceptable, results. Nevertheless, in cases where PFA fixation and other alternative fixation procedures fail to achieve satisfying immunostainings of a target, it might be worth to test glyoxal.

Another issue that will arise from the use of glyoxal fixation for very specific immunostaining and imaging cases, is the lack of the possibility to compare results from these experiments with existing literature, where most likely PFA fixation was used. But this is a problem almost every new method faces until it is well enough established in the scientific community.

Imaging experiments during the fixation of cells expressing GFP-tagged proteins showed that the glyoxal solution reduces the fluorescence intensity of GFP drastically, probably caused by either the low $\mathrm{pH}$ or the amount of ethanol in the solution. This could potentially pose a problem, if this is due to changes in the GFP confirmation, which are irreversible, even after changing to a more physiological solution after fixation. But even if GFP fluorescence turns out to not be as efficient in glyoxal fixed samples as in PFA fixed samples, other fluorescent tags have been shown to work without problems, e.g. mOrange used for tagging vimentin in neuroglioma cells (see experiments from Tiago Outeiro's laboratory).

Another issue of glyoxal fixation that needs some adjustment is the handling of the fixed tissue. It has been reported that glyoxal fixed tissue is softer and thus might behave differently during embedding or other procedures that need more extensive handling. This however did not seem to be a major obstacle in the different comparison studies done in the collaborating laboratories.

Further concerns might be raised about the amount of ethanol that is needed in the glyoxal solution for optimal fixation. Ethanol in principle could lead to fixation by precipitation, but this is not the case for the relatively small amount of ethanol in the glyoxal mixture $(10-$ $20 \%$ ). We have shown that the ethanol is most likely not involved in the fixation process itself, since it does not improve the fixation of samples when added to PFA. Nevertheless, the presence of ethanol is likely to result in some lipid extraction from membranes, since it helps with faster penetration of cells. However, this lipid extraction is probably limited, which is suggested by the good preservation of membrane proteins after glyoxal fixation (see comparison results for membrane proteins in Figure 10).

Lastly, it has not been tested yet, whether ready-to-use glyoxal solutions can be prepared in advance and be frozen until further use, which is common scientific practice for PFA solutions. Until now, we have only tested freshly prepared glyoxal solutions. However, the glyoxal fixation solution is easy to prepare and poses much less of a health risk than preparing PFA solutions, since glyoxal is already sold in an aqueous solution and thus has almost no vapor pressure, which makes it less likely to be inhaled (Dapson, 2007). 
All advantages and disadvantages considered, glyoxal seems to be a good alternative fixative for samples that cannot be sufficiently fixed by PFA. It might need some additional testing for optimal use, but even in cases where it "only" provides similar fixation quality as PFA, it is worth using due to the decreased health risks.

\subsection{CosiQuant, a novel method to estimate protein copy numbers}

In the second part of my project, I established the method CosiQuant to estimate protein copy numbers by comparative imaging. The comparison of immunostaining signals obtained from biochemically characterized synaptosomes and a sample of interest permits the approximation of protein copy numbers in the sample of interest. This is especially beneficial for the investigation of samples, where proteins cannot be quantified by commonly used biochemical methods, due to the lack of sufficient purification and/or sample amount. The use of an imaging approach also adds improved spatial resolution, which in turn allows for distinguishing between specific cells, compartments or organelles. This is not the case for techniques like quantitative MS or Western Blotting, which only provide an overall average for the obtained protein numbers in a sample, containing multiple cells or layers of tissue. On the other hand, using an imaging approach for the investigation of protein numbers can only produce estimates rather than precise numbers, due to high variability in the signal quality. Further it has to be assumed that immunostaining intensities increase linearly with the amount of expressed proteins and that immunostaining efficiencies are equal in synaptosomes and the sample of interest. Especially the latter assumption has to be approached with caution, since the method is based on the immunostaining of a POI by primary and secondary antibodies. While being a very established and widely used method, the staining with fluorescently tagged antibodies has been shown to harbor several problems. First of all, due to their relatively big size (150 kDa, $10 \mathrm{~nm})$ the penetration of antibodies into a sample is known to be limited and varies between different samples (Maidorn et al, 2016). This might not pose a major problem for the comparison of immunostainings between samples that are similar in thickness and density, like cultured cells. But if samples are supposed to be compared that are substantially different, like the synaptosome preparations and tissue samples or even whole organs, penetration efficiency has to be tested and potential differences have to be controlled for. This can be done e.g. by expressing GFP-tagged proteins in the sample of interest and immunostaining either against the tag or the POI. Co-localization of the signals will then give an estimate of the penetration and overall immunostaining efficiency. Since this is quite labor-intensive, an alternative option would be to use sections of a tissue or organ instead of the whole organ for the comparison to synaptosomes. Using cryosections for example can reduce the sample thickness to $10 \mu \mathrm{m}$ and thus will facilitate antibody penetration immensely.

A similar problem, which is also caused by the size of the antibodies, is the epitope accessibility. If the epitope against which an antibody is raised is difficult to reach or partly masked, due to conformational changes dependent on interactions with other proteins or dependent on the environment, then the binding efficiency can vary. This is less of a problem concerning the comparability of antibody stainings between two samples, since the 
epitope accessibility should vary similarly in the two samples, if they are treated identically during the staining process. But the issue of epitope accessibility can overall limit the ability of investigating a certain protein, if no antibodies with reliable epitope recognition are available. The issue of varying immunostaining efficiencies, due to varying epitope recognition is further complicated by the use of polyclonal antibodies. Since a majority of available antibodies are polyclonal (i.e. they consist of a mixture of antibodies that recognize different epitopes of the same POI), it can be expected that these contribute to the variability of the staining efficiency. Therefore, it must be ensured that during immunostaining of the samples for CosiQuant, the same antibody mixture is used for both samples.

Probably the most crucial concern for the use of primary and secondary antibodies for quantification purposes is that the number of fluorescent labels per secondary antibody is often not directly controlled. The fact that secondary antibodies can have variable numbers of fluorophores and that primary antibodies can be bound by different amounts of divalent secondary antibodies, makes direct quantification by the use of antibodies almost impossible (Fornasiero \& Opazo, 2015). Therefore, in CosiQuant we compare relative differences in staining intensities between the samples investigated. Nevertheless, again it has to be ensured that staining conditions and especially antibody mixtures are as similar as possible, if not identical. Only then can it be assumed that measured differences in staining intensities represent real differences in protein expression and are not caused by variations in the staining efficiency due to the issues mentioned above.

An elegant solution to all those problems would be the use of nanobodies instead of antibodies as immunostaining probes. Due to their small size and monovalency, nanobodies have been reported to alleviate most of the disadvantages that antibodies face (Maidorn et al, 2016). Nanobodies only consist of the variable domain of the heavy chain antibody and are therefore significantly smaller $(15 \mathrm{kDa}, 2 \mathrm{~nm})$ than a common IgG antibody. The penetration of thick samples by nanobodies is therefore increased and epitopes can be accessed more easily. Additionally, nanobodies contain CDR3 regions (complementary determining regions) that are more variable in size than those of the commonly used antibodies. These regions determine the epitope binding, thus the more flexible CDR3 region of the nanobody increases epitope recognition and accessibility (Dmitriev et al, 2016). Nanobodies are also more suitable for quantification purposes, since they are monovalent (i.e. they only bind one epitope) and can be directly labeled relatively easy. The labeling with fluorescent dyes can be done in a controlled stoichiometric ratio, which provides the opportunity of more accurate protein quantification from signal intensities (Platonova et al, 2015). All these advantages would make nanobodies an ideal probe for the immunostaining of samples in CosiQuant. Unfortunately, so far nanobodies for only a few target proteins are available, due to complex selection and validation procedures during the production of new nanobodies. Once nanobodies are available for a variety of different target proteins, they can provide a major improvement for the CosiQuant technique.

Another issue, which is often associated with immunostainings and therefore needs to be considered in CosiQuant, is the presence of background signal. This so-called noise is caused 
by non-specific binding of affinity probes (antibodies, nanobodies or other probes used for immunostainings) to structures other than the target epitope. Blocking of those structures with solutions containing e.g. BSA or trypton peptone reduces the background signal, but often cannot eliminate it completely. Thus, the signal intensity of immunostainings used in CosiQuant needs to be normalized to the level of noise with appropriate control stainings, ideally in every comparative staining procedure. This increases the workload for the application of CosiQuant, but is a crucial step, which should not be omitted.

All of the problems concerning the use of immunostainings as a method for protein number estimations are important issues, but can be controlled for, as described above. Apart from these issues, CosiQuant exhibits some limitations that cannot be omitted so easily. Due to the fact that the method is based on proteins quantified in synaptosomes, CosiQuant cannot be applied to proteins that are specific and limited to certain other samples or tissues. The IHC marker protein otoferlin for example cannot be quantified using CosiQuant, since its expression is limited to IHCs and consequently there is no reference copy number in synaptosomes. The method is further limited by the need for synaptosome preparations that can be stained in parallel with the sample of interest. To facilitate the application of CosiQuant in other laboratories, which are not in possession of the synaptosome samples, we provided a substitute for the immunostaining intensity of synaptosomes. We have measured the average number of antibodies bound to a POI in synaptosome samples and provide a list of such average numbers of antibodies for over 100 POIs. This makes it possible to compare immunostaining signals in terms of numbers of bound antibodies and thus eliminates the direct need for synaptosome samples. Nevertheless, the POI still needs to be immunostained in the sample of interest under the exact same condition as the synaptosomes have been stained, i.e. using the same antibodies, permeabilization conditions and blocking conditions, although these conditions might not work for every sample of interest. In summary, CosiQuant is a semi-quantitative method that provides a solution for a specific range of samples rather than a broadly applicable technique which can be generalized easily. However, for some samples, such as the IHC, it can be of great use to acquire first estimates of protein copy numbers, which otherwise cannot be obtained, using the common biochemical methods.

\subsection{Investigation of proteins involved in the synaptic vesicle recycling process at the IHC ribbon synapse}

In the third and last part of my project, I combined the achievements of the first two parts to investigate the molecular anatomy of the IHC ribbon synapse. I implemented glyoxal fixation for the subsequent immunostaining of POIs in the ribbon synapse. I then determined the spatial organization of proteins potentially involved in synaptic vesicle exo- and endocytosis and estimated their copy numbers using CosiQuant. Investigating those parameters in the IHC ribbon synapse is of special interest with regard to the functional challenges these synapses face as first points of stimulus transduction in the auditory system. The IHC ribbon synapse needs to be capable of very fast stimulus-transmission coupling, precisely transmitting changes of the sound stimulus to afferent neurons and at the same time it has 
to maintain long-lasting transmission in response to persistent stimulation (Nouvian et al, 2006). These special features of the IHC ribbon synapse require a very efficient process of synaptic vesicle exo - and endocytosis. Synaptic vesicle exocytosis, and thus neurotransmitter release, needs to be very fast and precise in order to mimic changes in the sound stimulus. This is reflected in the capability of IHC ribbon synapses to release a very high number of vesicles within the first milliseconds of stimulation, exceeding by far the release rates of conventional synapses (Moser \& Beutner, 2000). This very fast exocytosis needs to be compensated by efficient synaptic vesicle recycling to ensure synaptic transmission beyond those first milliseconds. Thus, there is a need for ample endocytosis of synaptic vesicle material from the plasma membrane to guarantee sufficient vesicle replenishment. In principle, synaptic vesicle endocytosis can occur in two different modes, via clathrin-mediated endocytosis of vesicles or via so called bulk endocytosis, in which synaptic vesicles are reformed from large plasma membrane infoldings. Evidence for the existence of both modes of endocytosis has been reported at the IHC ribbon synapse (Siegel \& Brownell, 1986; Lenzi et al, 2002; Kamin et al, 2014; Neef et al, 2014). Based on capacitance measurements and inhibition of dynamin as well as clathrin, it has been suggested that clathrin-mediated endocytosis is the predominant mode of endocytosis during mild stimulation, whereas bulk endocytosis is probably responsible for fast vesicle recycling upon stronger stimulation (Neef et al, 2014). Both modes of endocytosis have been shown to take place close to the active zone of the ribbon synapse using electron microscopy (Siegel \& Brownell, 1986; Lenzi et al, 2002; Kamin et al, 2014; Neef et al, 2014). However, it is still an open question whether there are distinct areas of endocytosis, where proteins involved in the process are accumulated, like reported for the photoreceptor ribbon synapse (Wahl et al, 2013). The results from my investigation of synaptic proteins in the ribbon synapse area using super-resolution imaging contribute to the discussion of such questions. Using STED microscopy, I have determined the location of 7 endocytosis proteins and 12 proteins commonly associated with synaptic vesicle exocytosis or vesicle trafficking, in relation to the ribbon. Below I first want to discuss briefly the proteins I have investigated here, relating my observation to the knowledge from previous studies about the localization and abundance of these proteins. I will then discuss the estimated protein abundance in context to release rates comparing conventional synapses and the IHC ribbon synapse. Lastly, I want to address topics like the possible existence of a periactive zone for endocytosis and the still ongoing discussion about expression of conventional neuronal exocytosis SNARE molecules at the IHC ribbon synapse.

As mentioned above, in addition to 7 endocytosis proteins I have studied 12 proteins, involved in vesicle exocytosis and trafficking. Among these, 6 proteins are SNARE molecules of various cell compartments and 6 proteins are associated with the synaptic vesicle membrane, of which 5 are potentially involved in the process of synaptic vesicle exocytosis at the ribbon synapse.

By far the most abundant protein I determined in my study is the synaptic vesicle membrane protein rab3. It has already been detected in IHCs by different studies using MS and RNA 
sequencing (Uthaiah \& Hudspeth, 2010; Scheffer et al, 2015). My results can be taken as a validation of the expression of rab3 in IHCs by means of an imaging method. The high abundance of this small GTPase is not surprising, since rab3 is a common synaptic vesicle marker in neurons and has already been used as such in imaging studies of the IHC as well (Revelo et al, 2014). Rab3 has been shown to be localized throughout the whole IHC, despite the fact that ribbon synapses and thus synaptic vesicle exo- and endocytosis are concentrated at the base of the cells (Revelo et al, 2014). This omnipresence of rab3 fits the average distribution and high abundance of the protein that $\mathrm{I}$ have found in my investigations. It further suggests that vesicles containing rab3 are highly abundant not only in the ribbon area, but also at the apical part of the cell, where vesicle trafficking between endosomes and the golgi apparatus are predominant (Revelo et al, 2014).

Another synaptic vesicle membrane protein, CSP, seems to be expressed in relatively low numbers compared to rab3. Interestingly, synapses of efferent neurons close to the ribbon synapses seem to express higher amounts of CSP, since substantially brighter signals of CSP stainings were localized to synaptophysin containing synapses outside of the IHC (see Figure 4 of chapter 5). However, less bright immunostaining intensities for CSP were also detected in the ribbon area. The protein has already been reported to be expressed in IHCS by immunolabeling methods and MS (Uthaiah \& Hudspeth, 2010; Eybalin et al, 2002), which confirms my results. In conventional synapses, CSP is known to act as a chaperone to the SNARE protein SNAP25 (Sharma et al, 2011). Since the presence of the conventional neuronal exocytosis SNARE proteins, including SNAP25, at IHC ribbon synapses, is highly debatable, it is not clear which function CSP would have at the ribbon synapse, if SNAP25 is indeed not expressed there. The fact that CSP can be detected in the ribbon area either suggests that common neuronal exocytosis SNAREs are expressed at the IHC ribbon synapse, but cannot be detected with the conventional staining methods or it might suggest that CSP exhibits a different function in IHCs. In order to solve this question, further studies have to be conducted, focusing on the interaction of CSP and SNAP25 in IHCs.

The $\mathrm{Ca}^{2+}$ sensors synaptotagmin 1 and 2 are known to be expressed in IHCs during development, but have been declared as absent in IHCs after the onset of hearing (Safieddine \& Wenthold, 1999; Uthaiah \& Hudspeth, 2010; Wenthold et al, 2002; Beurg et al, 2010). My investigations of synaptotagmin 2 show protein expression at the ribbon synapse, albeit in very low amounts. Due to the ample evidence that synaptotagmin 1 and 2 are not expressed in adult IHC ribbon synapses, the detection here might be considered as a result of contamination or non-specific background signal, which has not been entirely corrected for. Another explanation might be the detection of residual synaptotagmin in IHCs, since the cells I investigated were taken from mice of young age (P14 - P18). Synaptotagmin 1 has been shown to exhibit a lifetime of about 10 days in mouse brains (Fornasiero et al, 2018) and thus it is possible that there is still a low amount of synaptotagmin in IHCs so close after the onset of hearing (around P12). This would be in agreement with the detection of low expression values for synaptotagmin 2 in adult IHCs using DNA microarrays (Liu et al, 2014b). Nevertheless, the detection of synaptotagmin 2 in my investigation has to be considered 
with caution, especially since the immunostainings of the individual POIs showed high variability.

Synaptotagmin 7 has been reported to regulate synaptic vesicle replenishment in neurons (Liu et al, 2014a) and therefore was an interesting target protein to investigate in IHCs, where vesicle replenishment has to be very efficient. Unfortunately, synaptotagmin 7 has been detected in even lower amounts than synaptotagmin 2 , thus it can only be speculated whether our synaptotagmin 7 detection reflects its real expression in IHCs, especially with regards to previous studies reporting that synaptotagmin 7 , just like synaptotagmin 2 , is only expressed in the IHC during development (Beurg et al, 2010; Safieddine et al, 2012).

Similar to CSP, I found high signal intensity for the vesicular proton pump vATPase predominantly outside of the IHC, most likely in efferent synapses. Still, a lower amount of signal can be found in the ribbon synapse area as well, suggesting that vATPase is also expressed in IHCs. The protein consists of several subunits and a few of them have been identified previously in MS, DNA microarray and RNA sequencing experiments (Uthaiah \& Hudspeth, 2010; Hickox et al, 2017; Scheffer et al, 2015; Liu et al, 2014b). The 116 kDa subunit investigated in my study is essential for the proton pump activity of the protein and shows intermediate to low abundancy compared to the other investigated proteins.

Synaptogyrin is the last protein I investigated, which might be involved in the exocytosis process of synaptic vesicles. The exact function of this protein is rather unclear even in conventional synapses, but there it has been reported to be associated with the synaptic vesicle (Südhof, 2004; Takamori et al, 2006). In IHCs, synaptogyrin has only been detected once by MS of proteins co-purified with ribbons. In that study, synaptogyrin was not considered for quantification, since it was detected only in trace amounts. This was probably due to the fact that co-immunoprecipitation with the ribbon only captured a few vesicles and thus synaptic vesicle proteins were underrepresented (Kantardzhieva et al, 2012). Nevertheless, it does suggest that synaptogyrin might be expressed at the ribbon synapse, although these results have to be interpreted with caution, since the study was completed with retina ribbons, as IHCS did not provide enough material to be analyzed. In my investigations, synaptogyrin is one of the proteins that has been identified in very low amounts, like synaptotagmin 2 and 7 . Therefore, my results concerning this protein do not provide enough evidence to confirm the expression of synaptogyrin and to make assumptions about the function in the synaptic vesicle recycling process at the IHC ribbon synapse.

Due to some evidence from previous studies reporting that the conventional neuronal exocytosis SNARE proteins syntaxin 1, SNAP25 and VAMP2 are not expressed in the IHC, I included some other SNARE proteins in my study, which might be able to compensate for the lack of those proteins at the ribbon synapse. These alternative SNARE proteins are syntaxin 6, syntaxin 12/13 and syntaxin 16, as well as VAMP3, VAMP4, and SNAP29. Those proteins are usually associated with vesicle trafficking between the golgi network and endosomes and interact with each other to mediate membrane fusion in the same fashion as the SNARE proteins mediating fusion at the conventional synapse. All three syntaxins investigated here have previously been detected in the IHC by MS, DNA microarray and 
reverse transcription PCR (Liu et al, 2014b; Uthaiah \& Hudspeth, 2010; Hickox et al, 2017; Safieddine \& Wenthold, 1999). Still, none of these studies were able to localize the proteins within the IHCS, due to the lack of spatial resolution. Precise localization is crucial for distinguishing between SNARE proteins involved in synaptic vesicle exocytosis and SNARE proteins involved in vesicle trafficking between organelles like the golgi network or endosomes. My investigations of the distribution and abundance of the syntaxin proteins at the ribbon synapse show only low levels of all three proteins at the ribbon, suggesting that they do not play a major role in synaptic vesicle exocytosis. This is very well in line with results from a study using super resolution imaging of syntaxin 6 and 16 in IHCs, divided in apical, nuclear and basal regions of the cell. Here, syntaxin 6 and syntaxin 16 were predominantly found in the apical and nuclear regions of the IHC. The basal part, containing the ribbon synapses, was almost completely devoid of the proteins (Revelo et al, 2014). Thus, the syntaxins investigated in my thesis seem to mediate vesicle fusion in parts of the cell other than the ribbon synapse and contribution to synaptic vesicle exocytosis seems limited, if at all. Similarly, SNAP29 localization and protein copy number estimation showed very low abundance of the protein at the ribbon synapse and therefore SNAP29 cannot be considered to function as an alternative synaptic SNARE protein to SNAP25.

Although VAMP3 has been identified in MS analysis of IHC samples (Uthaiah \& Hudspeth, 2010) and it has been found in retinal ribbon synapses (Von Kriegstein et al, 1999; Lenzi \& von Gersdorff, 2001), super-resolution imaging of the protein in my study does not suggest substantial expression or localization close to the ribbon. Protein copy numbers for VAMP3 in IHCS are still under investigation, since there are no reference copy numbers in synaptosomes available, yet. But the low immunostaining intensities for this protein suggest only little amounts at the ribbon synapse. VAMP4 has been found to interact with syntaxin 6 and 16 in trans-golgi network trafficking. Since I showed that syntaxin 6 and 16 do not play a major role in synaptic vesicle exocytosis at the ribbon synapses, it is no surprise that the interaction partner VAMP4 is also not found in any significant amount at the ribbon synapse of IHCs.

The investigation of endocytosis proteins proved to be more conclusive than that of SNARE proteins. The synaptic vesicle coat protein clathrin for example has been identified as the second most abundant protein at the ribbon synapse in my study. In previous studies using $E M$, clathrin coated pits and vesicles have been observed close to the ribbon synapse and the adaptor protein AP2, responsible for recruiting clathrin to the site of endocytosis, has been shown to be required for endocytosis in IHCs (Neef et al, 2014; Jung et al, 2015). As described before, clathrin-mediated endocytosis has been proposed to be the predominant mode of endocytosis upon mild stimulation, since clathrin and dynamin inhibition results in abolished endocytosis after mild stimulation of synapses. Due to sufficient evidence in the literature for the expression of clathrin in IHCs, it is reasonable to believe that the amount of clathrin I determined at the ribbon synapse reflects the functionally relevant pool of the protein for synaptic vesicle endocytosis.

I found the adaptor protein AP180 to be present at the ribbon synapse with a similar amount to clathrin. This is consistent with what is known about the function of AP180 in 
conventional synapses. There, it is responsible for the recruitment of clathrin to the synaptic vesicle, which is about to be endocytosed. Another such adaptor protein for the clathrin coat assembly is AP2, which has been found to play a major role in synaptic vesicle endocytosis in IHCs (Jung et al, 2015; Pangršič \& Vogl, 2018). It is therefore plausible to assume that AP180 is needed at the $\mathrm{IHC}$ ribbon synapse, too, although direct identification of the protein at the ribbon synapse has only been reported once before by MS (Uthaiah \& Hudspeth, 2010). In conventional synapses, AP180 binds VAMP molecules in the plasma membrane (Koo et al, 2011). Since VAMP1 and 2 (the major synaptic vesicle SNARE molecules in conventional synapses) have been proposed to not be expressed at the IHC synapse, it is an interesting question which protein is targeted by AP180 instead. The same question can be asked for AP2, since in neurons it has been shown to bind to synaptotagmin (Diril et al, 2006), another molecule, which has been reported to not be present in adult IHCs.

Further proteins known to mediate endocytosis of synaptic vesicles in conventional synapses are amphiphysin, endophilin and synaptojanin. Via interaction with each other and additional key endocytosis proteins, like clathrin and dynamin, they mediate the assembly and disassembly of the clathrin coat, synaptic vesicle membrane invagination and fission (Slepnev \& De Camilli, 2000; Haucke et al, 2011; Rizzoli, 2014). I have determined different amounts of each protein at the IHC ribbon synapse. While amphiphysin seems to be present in intermediate amounts compared to the other investigated proteins in this study, endophilin and especially synaptojanin show low copy number estimates. All three proteins have been detected in IHCs before by RNA sequencing and MS (Scheffer et al, 2015; Li et al, 2018; Uthaiah \& Hudspeth, 2010; Hickox et al, 2017). Additionally, amphiphysin has been visualized in IHCs using immunohistochemistry (Neef et al, 2014), confirming that amphiphysin is expressed in the IHC in amounts that can be detected by immunostainings. Endophilin on the other hand has been difficult to detect by immunostaining. Kroll and colleagues reported that endophilin could not be reliably immunolabeled in IHCs, despite the testing of different staining conditions and antibodies. Yet, the same study confirms endophilin expression in IHCs using single-cell RT-PCR and immunoblotting (Kroll et al, 2019). This illustrates that protein expression can be missed by immunolabeling experiments, if staining conditions are not optimal. Using glyoxal fixation, I was able to detect fluorescent signal from endophilin immunostainings, although to a lower amount in IHCS than in efferent synapses. Similarly, low imunolabeling intensities were observed for synaptojanin, which seems to be expressed only in low numbers at the IHC ribbon synapse. However, low abundancy does not indicate lower importance. In conventional synapses copy numbers of synaptojanin are low, too (Wilhelm et al, 2014), but the protein has already been shown to play an important role in synaptic vesicle endocytosis in neurons (Slepnev \& De Camilli, 2000). Further indication for the relevance of synaptojanin in hair cells comes from studies in zebrafish mutants, where the protein has been shown to be required for the synaptic vesicle recycling in neuromast hair cells (Trapani et al, 2009). Altogether, evidence from previous studies suggest that all three endocytosis proteins investigated in my study are involved in the synaptic vesicle recycling at the IHC ribbon synapse and thus validates the detection of those proteins in my study. 
Another protein tightly associated with clathrin mediated endocytosis is dynamin, a GTPase that mediates the fission of the clathrin coated vesicle from the plasma membrane (Hinshaw, 2000; Faelber et al, 2012). Since various other key proteins of the clathrin mediated endocytosis process have already been detected in the IHC ribbon synapse, it is likely that dynamin is expressed here as well. Indeed, I found dynamin present at the ribbon synapse in similar amounts to amphiphysin, which is one of the proteins known to recruit dynamin to vesicles at the plasma membrane (Slepnev \& De Camilli, 2000; Haucke et al, 2011). Confirmation of dynamin being expressed at the IHC ribbon synapse and being involved in synaptic vesicle recycling in those synapses is provided by previous studies. The RNA of dynamin has been detected in RNA sequencing analyses (Li et al, 2018) and the protein has been detected by MS, immunofluorescence experiments, immunoblotting and immunoprecipitation (Uthaiah \& Hudspeth, 2010). The functional relevance of the protein has been shown, like mentioned earlier, in a study inhibiting dynamin, which resulted in the impairment of clathrin mediated endocytosis at the IHC ribbon synapse (Neef et al, 2014).

The expression of SCAMP1, the last protein I have investigated in my study, at the ribbon synapse is not as evident as for dynamin or clathrin. In neurons, SCAMP1 is involved in general recycling processes from the plasma membrane (Südhof, 1995), but is not as tightly linked to synaptic vesicle endocytosis as the proteins described above. That might be the reason why SCAMP1 has not been investigated as much as the other proteins in the IHC ribbon synapse. Its presence has only been described once by MS and immunoblotting analyses of IHCs (Uthaiah \& Hudspeth, 2010). I have found SCAMP1 to be expressed at the ribbon synapse of my samples in relatively low amounts, similar to the vATPase. Again, high abundancy does not correlate with importance of the protein in a specific process, but it might suggest whether a protein presents a rate-limiting factor of that process. This is probably not the case for SCAMP1, since I have found other proteins, crucial for the synaptic vesicle exo- and endocytosis, to be expressed in even lower amounts than SCAMP1, like endophilin or synaptojanin.

\subsection{Relating protein copy numbers to synaptic vesicle release kinetics}

In general, most of the proteins investigated in my thesis seem to be expressed in substantially higher amounts compared to the expression of those proteins in conventional synapses of neurons. Proteins like rab3 or clathrin for example have been detected with a 100 - 200 times higher abundance in the ribbon synapse of IHCs. These numbers are affected by the bigger size of the ribbon synapse compared to the conventional synapse, but most likely also by the increased need for synaptic vesicle recycling in IHCs. One can relate the amount of exo- and endocytosis proteins to the number of vesicles in the respective synapse in order to get an estimate for the efficiency of synaptic vesicle exo- and endocytosis. In conventional synapses, it has been shown that endocytosis proteins are far less abundant than exocytosis proteins (Wilhelm et al, 2014). While the conventional neuronal exocytosis SNARE proteins for example are present with an average copy number of 60 - 80 proteins per synaptic vesicle, key proteins for the endocytosis process, like clathrin and dynamin, show only $5-10$ copies per synaptic vesicle. The vesicular proton 
pump and amphiphysin exhibit even lower copy numbers, with only 2 and 3 proteins per synaptic vesicle, respectively (Wilhelm et al, 2014). These numbers show that the ratelimiting part of sustained synaptic vesicle transmission in conventional synapses is the recycling of vesicles via endocytosis from the plasma membrane rather than the exocytosis. Nevertheless, the amount of endocytosis proteins stated above is obviously sufficient for the release and recycling of the RRP of vesicles. Assuming that 150-180 copies of clathrin and 52 copies of dynamin are needed for the recycling of one vesicle (Wilhelm et al, 2014; Cheng et al, 2007; McMahon \& Boucrot, 2011; Shnyrova et al, 2013), then the amounts of these proteins at the synapse are sufficient to recycle roughly 25 and 40 vesicles, respectively. This resembles about $7 \%$ and $11 \%$ of the total vesicle pool (384 vesicles per synaptosome (Wilhelm et al, 2014)), which in turn corresponds to the size of the RRP in conventional synapses (RRP $=5 \%$ of vesicles determined in hippocampal neurons (Rizzoli \& Betz, 2005)), which is released during the first seconds of stimulation.

In comparison, the ribbon synapse of IHCS has to handle much higher release rates. The exocytosis of about 14 vesicles in the first few milliseconds is thought to represent the initial release of the RRP at the IHC ribbon synapse (Moser \& Beutner, 2000). Furthermore, synaptic vesicle release can be maintained with sustained stimulation over several seconds, exhibiting slower but steady release rates. Nevertheless, the synaptic vesicle pool of the IHC ribbon synapse on average is not larger than the pool determined for synaptosomes, assuming that the $100-200$ synaptic vesicles associated with the ribbon (Khimich et al, 2005) represent the physiologically relevant pool for vesicle release at the ribbon synapse. This number correlates roughly with the amount of vesicles we have counted in our ribbon synapse model, based on EM data (225 vesicles in the area of approx. $650 \mathrm{~nm} \times 815 \mathrm{~nm}$ around the ribbon). It is known that additional vesicles can be found in the cytosol around the ribbon, but that are not associated to the ribbon (Nouvian et al, 2006). So far, it is not clear if these vesicles participate in synaptic vesicle recycling, but the presence of those vesicles explains the slightly higher number of vesicles in our model compared to the literature. Since I have counted all discernable vesicles in the imaged area around the ribbon, not only the ribbon associated ones, I probably have included some of the cytosolic vesicles as well. Taking the pool sizes of synaptic vesicles in synaptosomes and IHC ribbon synapses into account, one can compare the amounts of exo- and endocytosis proteins per synaptic vesicle between the two synapse types (Table 1). As mentioned before, all investigated proteins here show higher copy number estimates for the ribbon synapse, thus the amount of those proteins per synaptic vesicle should be sufficient for synaptic vesicle recycling as seen in the synaptosomes. Like described above, the presence of about 9 clathrin molecules per synaptic vesicle seems to be enough for efficient synaptic vesicle recycling in synaptosomes. In the IHC ribbon synapse, on average 175 clathrin molecules are present per synaptic vesicle, which seems more than sufficient compared to synaptosomes. Proteins like the endosomal SNAREs on the other hand are only expressed in very low amounts in both synapses, with protein copy numbers of less than 1 per vesicle. This means that these proteins are either not relevant for the synaptic vesicle recycling process or they have to be accumulated very precisely at the location where they are needed. I could not 
observe such a precisely localized accumulation of the investigated SNAREs using superresolution imaging in the ribbon area. Thus, I assume that the investigated SNARE proteins are not crucial for synaptic vesicle recycling in $\mathrm{IHC}$ ribbon synapses.

As described above, most of the common key proteins for synaptic vesicle exo- and endocytosis, like rab3, clathrin, AP180 and dynamin, show a higher abundance per synaptic vesicle in the ribbon synapse compared to synaptosomes. This increased abundancy suggests that the proteins are present in sufficient amounts for a synaptic vesicle recycling process with similar rates to the ones observed in synaptosomes. However, taking the different release rates in synaptosomes and ribbon synapses into account, the issue becomes more complex. In IHCs, at least two different release rates have been measured. The initial fast release of the RRP of vesicles shows a release rate of about 14 vesicles within the first 10 ms of stimulation (Moser \& Beutner, 2000). This would mean a release of about 1400 vesicles per second, if the initial release rate would be maintained over time. This is not the case, since upon long-lasting strong stimulation it has been shown that release rates decrease to about 680 vesicles per second after the initial release of the RRP (Pangršič et al, 2010). Nevertheless, this release rate is still several orders of magnitude higher than the release rate for conventional synapses. Due to this difference in release rates, it is more conclusive to compare the estimated protein copy numbers in relation to the number of recycling vesicles in synaptosomes and $\mathrm{IHC}$ ribbon synapses. The two very different release rates at the ribbon synapse are distinct features of the IHC synapse. This is why I decided to compare both release rates separately with the average release rate of conventional synapses (Table 1).

Comparing the protein copy numbers per amount of vesicles released from the RRP, it seems that most of the investigated proteins are present at the ribbon synapse in sufficient amounts for synaptic vesicle exo- and endocytosis of the RRP. With the exception of synaptogyrin, all proteins show copy numbers per recycling vesicle similar or higher than seen in synaptosomes. However, this initial release of vesicles is only maintained for the first few milliseconds of stimulation in IHC ribbon synapses. With ongoing stimulation, about 680 vesicles per second are released from the ribbon synapse (Pangršič et al, 2010). Thus, comparing the amount of proteins per recycling vesicle per second between conventional synapses and IHC ribbon synapses, shows a different picture. Here, the amounts of all investigated proteins per recycling vesicle per second are substantially less in IHC ribbon synapses than in conventional synapses. With the release of an amount of vesicles more than 3 times the pool size of ribbon associated vesicles, the presence of the common exoand endocytosis proteins does not seem to be sufficient for compensation via the common synaptic vesicle recycling process.

It has been proposed that vesicle exocytosis exceeding the amount of vesicles from the RRP is compensated by bulk endocytosis (Neef et al, 2014). Large infoldings of the plasma membrane form as a result of the high number of vesicles released, and new synaptic vesicles can be pinched off from these membrane infoldings, possibly via clathrin-mediated endocytosis. This might take place at areas further away from the ribbon and thus can explain why endocytosis proteins located directly at the ribbon were measured in sub- 
sufficient amounts. Another theory concerning the potential mechanism for maintenance of high vesicle release rates has been discussed by Nouvian et al, 2006. It involves the recruitment of the so called "outlying" vesicles from the cytosol further away from the ribbon. It has been suggested that such vesicles participate in extrasynaptic exocytosis, allowing for a higher amount of vesicles exocytosed during strong stimulation (Nouvian et al, 2006; Lenzi et al, 1999, 2002; Khimich et al, 2005; Beutner et al, 2001). This would also explain why the amounts of exo- and endocytosis proteins measured directly at the ribbon synapse cannot account for the high release rates of sustained synaptic transmission.

In summary, synaptic vesicle exo- and especially endocytosis are complex processes at the $\mathrm{IHC}$ ribbon synapse. The estimates of copy numbers for various commonly known exo- and endocytosis proteins from my work provide support for existing theories to explain the underlying mechanisms of the synaptic vesicle recycling process. Release of synaptic vesicles from the RRP happens within the first milliseconds of stimulation and the amount of exoand endocytosis proteins present at the ribbon synapse is sufficient to mediate release and compensatory endocytosis of the vesicles. Sustained stimulation results in slower but still massive release of synaptic vesicles, which cannot be accomplished solely by the exo- and endocytosis of vesicles associated with the ribbon synapse. Neither the amount of ribbonassociated vesicles nor the amount of exo- and endocytosis proteins found in the ribbon area can account for the high release rates. This suggests that alternative mechanisms take place at the IHC ribbon synapse upon strong sustained stimulation in addition to the commonly known synaptic vesicle recycling process. These alternatives might involve mechanisms such as bulk endocytosis and/or extrasynaptic release, as suggested in the literature.

Table 1 Comparison of protein copy number estimates for synaptosomes and IHC ribbon synapses. The estimated copy numbers for the POls investigated in my study are compared between synaptosomes and $\mathrm{IHC}$ ribbon synapses in relation to the number of synaptic vesicles.

The copy number per ribbon synapse refers to the area around the ribbon, analyzed in EM images $(650 \mathrm{~nm} \times 815 \mathrm{~nm})$. The average number of synaptic vesicles in synaptosomes is 384 (from Wilhelm et al, 2014). Approximation of the number of synaptic vesicles in the ribbon synapse is 225 (counted from EM and in agreement with the literature (Khimich et al, 2005)). The number of recycling vesicles per second from the RRP in synaptosomes is 10 (estimation of RRP as $5 \% \approx 20$ vesicles released per 2 seconds (from Rizzoli \& Betz, 2005). The number of recycling vesicles per few milliseconds from the RRP in ribbon synapses is 14 (from Moser \& Beutner, 2000). The number of recycling vesicles per second from sustained release in ribbon synapses is 680 (from Pangršič et al, 2010).

SV - synaptic vesicle; syn - synaptosome; RS - ribbon synapse

\begin{tabular}{|c|c|c|c|c|c|c|c|}
\hline protein & $\begin{array}{c}\text { copy number } \\
\text { per } \\
\text { synaptosome }\end{array}$ & $\begin{array}{c}\text { copy } \\
\text { number } \\
\text { per } \\
\text { ribbon } \\
\text { synapse }\end{array}$ & $\begin{array}{c}\text { copy } \\
\text { number } \\
\text { per SV } \\
\text { (syn) }\end{array}$ & $\begin{array}{c}\text { copy } \\
\text { number } \\
\text { per SV } \\
\text { (RS) }\end{array}$ & $\begin{array}{c}\text { copy } \\
\text { number/re- } \\
\text { cycling SV } \\
\text { (per sec) in } \\
\text { syn (RRP) }\end{array}$ & $\begin{array}{c}\text { copy } \\
\text { number/re- } \\
\text { cycling SV } \\
\text { (few ms) in } \\
\text { RS (RRP) }\end{array}$ & $\begin{array}{c}\text { cycling SV (per } \\
\text { number/re- in RS } \\
\text { sec } \\
\text { (sustained } \\
\text { release) }\end{array}$ \\
\hline \hline $\begin{array}{c}\text { Amphi- } \\
\text { physin }\end{array}$ & 1194.2 & 10218.7 & 3.1 & 45.4 & 119 & 729.9 & 15 \\
\hline AP180 & 3736.4 & 27930.5 & 9.7 & 124.1 & 374 & 1995 & 41.1 \\
\hline $\begin{array}{c}\text { Clathrin } \\
\text { heavy }\end{array}$ & 3472.47 & 39366.1 & 9 & 175 & 347 & 2811.9 & 57.9 \\
\hline
\end{tabular}




\begin{tabular}{|c|c|c|c|c|c|c|c|} 
chain & & & & & & \\
\hline CSP & 941.18 & 1413.7 & 2.5 & 6.3 & 94 & 101 & 2.1 \\
\hline Dynamin & 2326.4 & 11036 & 6.1 & 49 & 233 & 788.3 & 16.2 \\
\hline $\begin{array}{c}\text { Endo- } \\
\text { philin }\end{array}$ & 2524.4 & 5702.9 & 6.6 & 25.3 & 252 & 407.4 & 8.4 \\
\hline Rab3 & 18846.58 & 105017.3 & 49.1 & 466.7 & 1885 & 7501.2 & 154.4 \\
\hline SCAMP1 & 1459.5 & 7161.6 & 3.8 & 31.8 & 146 & 511.5 & 10.5 \\
\hline SNAP29 & 77.47 & 150.6 & 0.2 & 0.7 & 8 & 10.8 & 0.2 \\
\hline $\begin{array}{c}\text { Synapto- } \\
\text { gyrin }\end{array}$ & 1854.8 & 2385.5 & 4.8 & 10.6 & 185 & 170.4 & 3.5 \\
\hline $\begin{array}{c}\text { Synapto- } \\
\text { janin }\end{array}$ & 365.61 & 1276.9 & 1 & 5.7 & 37 & 91.2 & 1.9 \\
\hline $\begin{array}{c}\text { Synapto- } \\
\text { tagmin 2 }\end{array}$ & 297.28 & 1620.9 & 0.8 & 7.2 & 30 & 115.8 & 2.4 \\
\hline $\begin{array}{c}\text { Synapto- } \\
\text { tagmin 7 }\end{array}$ & 182.64 & 617.6 & 0.5 & 2.7 & 18 & 44.1 & 0.9 \\
\hline $\begin{array}{c}\text { Syntaxin } \\
12 / 13\end{array}$ & 157.83 & 205.4 & 0.4 & 0.9 & 16 & 14.7 & 0.3 \\
\hline $\begin{array}{c}\text { Syntaxin } \\
16\end{array}$ & 91.27 & 1417.2 & 0.2 & 6.3 & 9 & 101.2 & 2.1 \\
\hline $\begin{array}{c}\text { Syntaxin } \\
6\end{array}$ & 121.67 & 3024.2 & 0.3 & 13.4 & 12 & 216 & 4.4 \\
\hline VAMP4 & 100.59 & 308.3 & 0.3 & 1.4 & 10 & 22 & 0.5 \\
\hline vATPase & 742.37 & 7868.6 & 1.9 & 35 & 74 & 562 & 11.6 \\
\hline
\end{tabular}

Besides the estimates of protein copy numbers, my work provides additional information that can add to the discussion of further issues concerning the IHC ribbon synapse. One such issue involves the question whether clathrin-mediated synaptic vesicle endocytosis is localized to special areas, where endocytosis proteins are accumulated. Such a spatial separation of exo and endocytosis could influence the efficiency of the recycling process, since the availability of specific proteins could be managed locally rather than over the whole synaptic area. As mentioned before, the existence of such periactive zones has been reported for ribbon synapses in retinal photoreceptors (Wahl et al, 2013) and is under discussion for IHC ribbon synapses. I have here investigated several common endocytosis proteins using super-resolution microscopy to localize these proteins in relation to the ribbon. This information can be used to check for areas around the ribbon that show localization of predominantly endocytosis proteins. Comparisons of the average distribution maps of 6 investigated endocytosis proteins, does not provide any evidence for the existence of a periactive zone containing accumulations of the investigated proteins (Figure 11). The endocytosis proteins seem rather evenly distributed around the ribbon and even for proteins that do show accumulation in specific areas, like AP180 or amphiphysin, these locations do not coincide with the enrichment of other endocytosis proteins. Therefore, my data suggests that there is no obvious periactive zone for endocytosis in IHCs. This assumption is supported by a previous study, investigating the localization of immunostained amphiphysin, clathrin and dynamin at the IHC ribbon synapse (Neef et al, 2014). Since it was not my primary intention to study the possible existence of a pericative zone, the comparison of averaged immunostaining signals is not the optimal technique to 
examine this issue. To investigate this matter in more detail, one would have to analyze the amount of colocalization of various endocytosis proteins directly, i.e. in direct co-staining experiments, rather than comparing average distributions of each protein individually. Nevertheless, my imaging data in combination with results from earlier studies, suggest that no such periactive zone exists in the area of $3 \times 3 \mu \mathrm{m}$ around the ribbon.

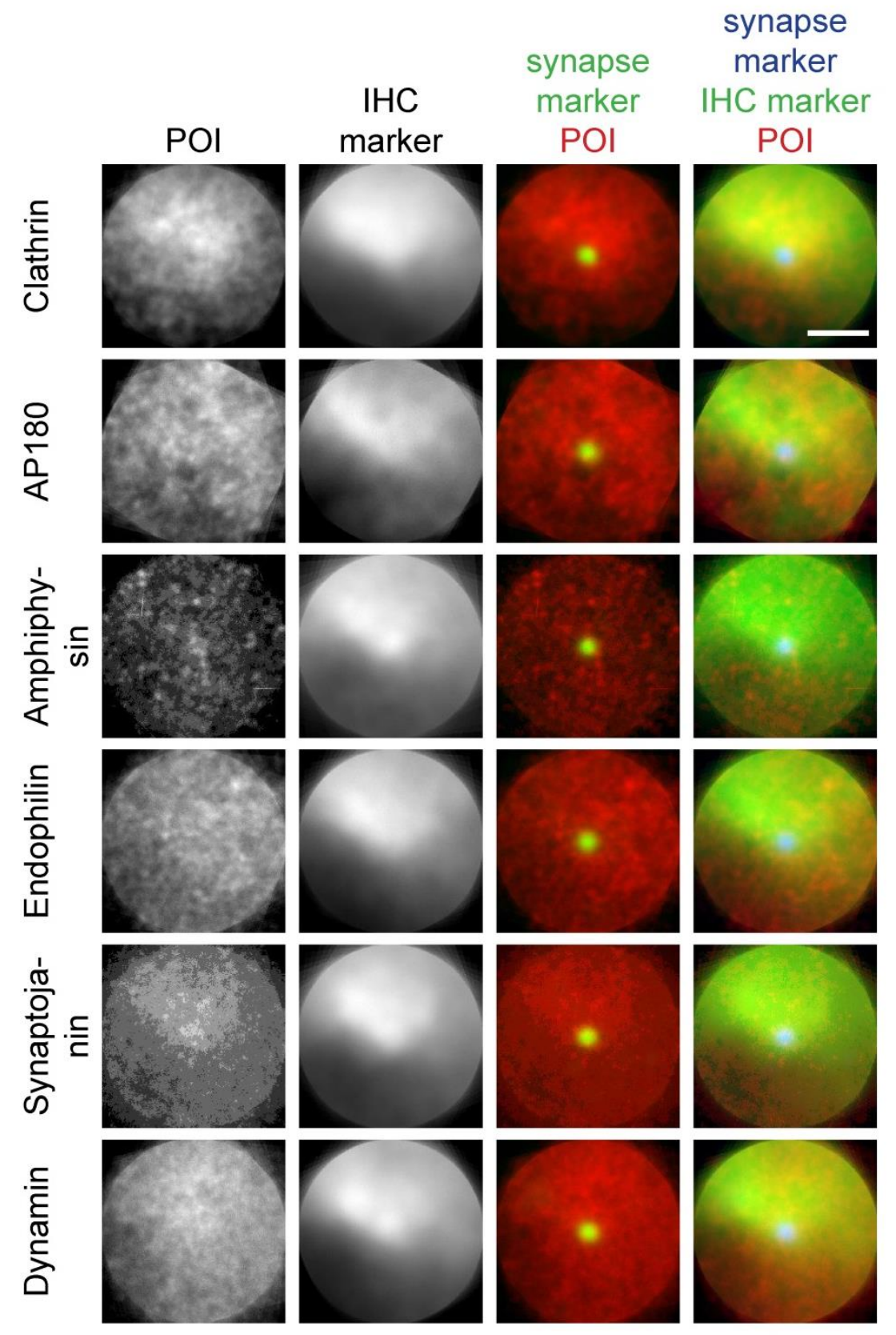

Figure 11 Average distribution of common endocytosis proteins in relation to the ribbon.

Average distribution maps (the same as in Figure 3 of chapter 05) are shown for the 6 common endocytosis proteins investigated in this study. The average locations of the POls in relation to the ribbon (CtBP2 or ribeye staining, third column) and additionally in relation to the cell outline (defined by vGlut3 staining as IHC marker, fourth column) can be compared. No obvious mutual areas of protein accumulation around the ribbon can be observed. Scale bar $=1 \mu \mathrm{m}$.

Another highly discussed issue, which I have touched upon briefly before in my work, is the expression of conventional neuronal exocytosis SNARE molecules in the IHC ribbon synapse. 
As described before, evidence for both the expression and the absence of SNAP25, syntaxin 1 and VAMP1/2 have been reported in the past (Nouvian et al, 2011; Uthaiah \& Hudspeth, 2010; Safieddine \& Wenthold, 1999). While Uthaiah and Hudspeth as well as Safieddine and Wenthold declare to be able to detect said proteins via immunohistochemistry, Nouvian and collegues describe the opposite. Furthermore, they show that inhibition of the proteins via botolinum neurotoxin as well as genetic ablation of the proteins does not have significant effects on synaptic vesicle exocytosis. Proving the presence or absence of a protein by immunohistochemistry alone is impossible, since different staining conditions and usage of different antibodies can have substantially diverse effects on the outcome of the staining quality. Proteins might be detected in one study, but not in the other, due to differences in the staining and also imaging procedure. Therefore, extensive testing of the different staining conditions is needed and even then immunohistochemistry experiments can only give suggestions as to whether a protein is expressed in a certain cell or organelle. On the other hand, pharmacological and genetic blocking of proteins can also not provide certainty for studying the expression of a protein. Effects of the inhibition might be masked by proteins with redundant functions. Thus, it can be difficult to provide enough evidence for the presence or absence of a protein and this evidence always needs to be validated with different experiments. The results of my immunostaining and super-resolution imaging experiments add to this collection of evidence for the presence or absence of common neuronal SNARE proteins at the IHC ribbon synapse. Using a new fixation method and testing different antibodies I was also not able to detect any significant immunostaining signal for SNAP25, syntaxin 1 or VAMP1/2, which strengthens the theory of Nouvian and collegues that the common neuronal exocytosis SNAREs are not expressed at the IHC ribbon synapse, at least not to an amount detectable by immunohistochemistry. Unfortunately, I was not able to provide any evidence for the expression of alternative SNARE molecules (e.g. SNAP29, syntaxin 6 or 12/13 or 16, VAMP3 or 4) at the ribbon synapse either. Furthermore, it seems like an interaction partner of SNAP25, CSP, is expressed at the ribbon synapse, which gives rise to the question, which function CSP has at the IHC synapse, if it's not acting as a chaperone to SNAP25, as in conventional synapses. But the same question arises for the adaptor proteins AP2 and AP180, which are expressed at the IHC ribbon synapse, but are missing their interaction partners synaptotagmin and VAMP2. In summary, whether conventional neuronal exocytosis SNARE proteins are expressed at the IHC ribbon synapse or not is still not clear, but the results of my imaging experiments add to the evidence that the latter is the case.

\subsection{Outlook}

Taking together the results of all three parts of my work, I have achieved to establish two technical improvements for the analysis of biological samples using fluorescence microscopy. One comprises the enhancement of immunostaining quality by glyoxal fixation as alternative to PFA fixation for super-resolution imaging techniques. The second achievement involves the establishment of a comparative imaging technique for the 
estimation of protein copy numbers (CosiQuant). Furthermore, by implementing these two methods for the analysis of the molecular anatomy of the synaptic vesicle recycling process at the IHC ribbon synapse, I was able to validate the applicability and the importance of these improvements especially for samples, which so far have been difficult to analyze by immunohistochemistry and super-resolution imaging. Additionally, with the analysis of the IHC ribbon synapse, I was able to provide a preliminary model of an average of such a synapse, containing the precise localization and protein copy number estimates of 19 synaptic proteins. This model is far from being complete, since it most likely only comprises a small amount of the proteins that are present at the IHC ribbon synapse, but it provides a backbone for the addition of more detailed information in order to create a comprehensive model of the average IHC ribbon synapse in the future. Data on the localization and abundance of additional proteins can be added as well as more detailed information about the already investigated proteins. These might comprise more precise copy numbers, interaction partners or functional details, which might be obtained by various different methods like gene knock out studies, in vitro studies of isolated proteins or improved imaging techniques, using e.g. nanobodies. Combinatorial stainings and imaging of proteins with the membrane marker mCling (Revelo et al, 2014) might also provide more insight into the synaptic vesicle recycling process, since exo- and endocytosis processes can be visualized and co-localization with respective POIs can be investigated. Improvements of existing techniques and the development of new methods can help to optimize the model over time. Recently, the imaging of retinal organoids has been achieved using an optimized tissue clearing protocol (Cora et al, 2019). Retinal tissue has been generated from pluripotent stem cells and ribbon synapses from the resulting organoids were imaged after tissue clearing. The study reports increased antibody penetration and specificity, thus enhancing high resolution imaging, while maintaining the 3-dimensional information of the whole organ. This application could be interesting for the Organ of Corti, since antibody penetration and specificity are major challenges for the imaging of IHCs. In my investigations, I used cryosectioning of the Organ of Corti in order to reduce these problems. However, this means that I am only able to image a section of the IHC and I am losing information about the whole 3-dimensional space. If the imaging of cleared organoids could be applied to Organs of Corti, this would alleviate this problem and could add valuable information to the IHC model. Additionally, one could extend the CosiQuant method by using synthetized POls in known amounts as reference for protein copy numbers. These synthetized proteins could be purified and added to the samples prior to comparative imaging and would function as internal standard. This would enable us to investigate proteins, which are important in IHCs, but not expressed in synaptosomes, such as otoferlin. Another interesting addition to the model of the average IHC ribbon synapse would be the investigation of the ribbon synapse in different states or variations. Differences in protein abundance and localization could be studied dependent on the activity state of the synapse, i.e. synapses at rest and under different stimulation conditions. Furthermore, differences of the protein composition could be tested between IHC ribbon synapses of the modiolar and pillar side or between synapses from IHCs residing at the apex and at the base of the cochlea. These investigations of the IHC 
ribbon synapses under different conditions can be done relatively easy in the near future, using the techniques I have established in my work. 


\section{$07 \mid$ References}

Advani RJ, Bae HR, Bock JB, Chao DS, Doung YC, Prekeris R, Yoo JS \& Scheller RH (1998) Seven novel mammalian SNARE proteins localize to distinct membrane compartments. J. Biol. Chem. 273: 10317-24

Bai J, Hu Z, Dittman JS, Pym ECG \& Kaplan JM (2010) Endophilin functions as a membranebending molecule and is delivered to endocytic zones by exocytosis. Cell 143: 430-41

Beaudoin GMJ, Lee S-H, Singh D, Yuan Y, Ng Y-G, Reichardt LF \& Arikkath J (2012) Culturing pyramidal neurons from the early postnatal mouse hippocampus and cortex. Nat. Protoc. 7: 1741-54

Becker L, Schnee ME, Niwa M, Sun W, Maxeiner S, Talaei S, Kachar B, Rutherford MA \& Ricci AJ (2018) The presynaptic ribbon maintains vesicle populations at the hair cell afferent fiber synapse. Elife 7: 1-26

Bethani I, Lang T, Geumann U, Sieber JJ, Jahn R \& Rizzoli SO (2007) The specificity of SNARE pairing in biological membranes is mediated by both proof-reading and spatial segregation. EMBO J. 26: 3981-92

Betz WJ, Mao F \& Bewick GS (1992) Activity-dependent fluorescent staining and destaining of living vertebrate motor nerve terminals. J. Neurosci. 12: 363-75

Beurg M, Michalski N, Safieddine S, Bouleau Y, Schneggenburger R, Chapman ER, Petit C \& Dulon D (2010) Control of exocytosis by synaptotagmins and otoferlin in auditory hair cells. J. Neurosci. 30: 13281-90

Beutner D, Voets T, Neher E \& Moser T (2001) Calcium Dependence of Exocytosis and Endocytosis at the Cochlear Inner Hair Cell Afferent Synapse. 29: 681-690

Bock JB, Klumperman J, Davanger S \& Scheller RH (1997) Syntaxin 6 functions in trans-Golgi network vesicle trafficking. Mol. Biol. Cell 8: 1261-71

Boucher J, Simard É, Froehlich U, D’Orléans-Juste P \& Grandbois M (2015) Using carboxyfluorescein diacetate succinimidyl ester to monitor intracellular protein glycation. Anal. Biochem. 478: 73-81

Brandt A, Striessnig J \& Moser T (2003) CaV1.3 channels are essential for development and presynaptic activity of cochlear inner hair cells. J. Neurosci. 23: 10832-40

Brunelle JL \& Green R (2014) One-dimensional SDS-polyacrylamide gel electrophoresis (1D SDS-PAGE). 1st ed. Elsevier Inc.

Caprioli RM, Farmer TB \& Gile J (1997) Molecular imaging of biological samples: localization of peptides and proteins using MALDI-TOF MS. Anal. Chem. 69: 4751-60

Carson F (2007) Histotechnology 2nd edition. Chicago: ASCP Press

Chakrabarti R, Michanski S \& Wichmann C (2018) Vesicle sub-pool organization at inner hair cell ribbon synapses. EMBO Rep. 19: e44937

Chamberlain LH \& Burgoyne RD (1997) The molecular chaperone function of the secretory vesicle cysteine string proteins. J. Biol. Chem. 272: 31420-6

Chapochnikov NM, Takago H, Huang C-H, Pangršič T, Khimich D, Neef J, Auge E, Göttfert F, Hell SW, Wichmann C, Wolf F \& Moser T (2014) Uniquantal release through a dynamic fusion pore is a candidate mechanism of hair cell exocytosis. Neuron 83: 1389-403

Charneau P, Mirambeau G, Roux P, Paulous S, Buc H CF (1994) HIV-1 reverse transcription. A termination step at the center of the genome. J Mol Biol. 241: 651-62

Cheng Y, Boll W, Kirchhausen T, Harrison SC \& Walz T (2007) Cryo-electron tomography of clathrin-coated vesicles: structural implications for coat assembly. J. Mol. Biol. 365: 
892-9

Cooper NP \& Guinan JJ (2006) Efferent-mediated control of basilar membrane motion. J. Physiol. 576: 49-54

Cora V, Haderspeck J, Antkowiak L, Mattheus U, Neckel PH, Mack AF, Bolz S, Ueffing M, Pashkovskaia N, Achberger K \& Liebau S (2019) A Cleared View on Retinal Organoids. Cells 8: 391

Cremona O \& De Camilli P (1997) Synaptic vesicle endocytosis. Curr. Opin. Neurobiol. 7: 32330

Dapson RW (2007) Glyoxal fixation: how it works and why it only occasionally needs antigen retrieval. Biotech. Histochem. 82: 161-6

Davey HM \& Kell DB (1996) Flow cytometry and cell sorting of heterogeneous microbial populations: the importance of single-cell analyses. Microbiol. Rev. 60: 641-96

Denker A, Kröhnert K, Bückers J, Neher E \& Rizzoli SO (2011) The reserve pool of synaptic vesicles acts as a buffer for proteins involved in synaptic vesicle recycling. Proc. Natl. Acad. Sci. U. S. A. 108: 17183-8

Dick O, Hack I, Altrock WD, Garner CC, Gundelfinger ED \& Brandstätter JH (2001) Localization of the presynaptic cytomatrix protein Piccolo at ribbon and conventional synapses in the rat retina: comparison with Bassoon. J. Comp. Neurol. 439: 224-34

Dick O, tom Dieck S, Altrock WD, Ammermüller J, Weiler R, Garner CC, Gundelfinger ED \& Brandstätter JH (2003) The presynaptic active zone protein bassoon is essential for photoreceptor ribbon synapse formation in the retina. Neuron 37: 775-86

Diril MK, Wienisch M, Jung N, Klingauf J \& Haucke V (2006) Stonin 2 is an AP-2-dependent endocytic sorting adaptor for synaptotagmin internalization and recycling. Dev. Cell 10: 233-44

Dmitriev OY, Lutsenko S \& Muyldermans S (2016) Nanobodies as Probes for Protein Dynamics in Vitro and in Cells. J. Biol. Chem. 291: 3767-75

Dou H, Vazquez AE, Namkung Y, Chu H, Cardell E Lou, Nie L, Parson S, Shin H-S \& Yamoah EN (2004) Null mutation of alpha1D Ca2+ channel gene results in deafness but no vestibular defect in mice. J. Assoc. Res. Otolaryngol. 5: 215-26

Drury R \& Wallington E (1980) Carleton's histological technique 5th ed. New York: Churchill Livingstone

Dulon D, Safieddine S, Jones SM \& Petit C (2009) Otoferlin is critical for a highly sensitive and linear calcium-dependent exocytosis at vestibular hair cell ribbon synapses. J. Neurosci. 29: $10474-87$

Duncker S V, Franz C, Kuhn S, Schulte U, Campanelli D, Brandt N, Hirt B, Fakler B, Blin N, Ruth P, Engel J, Marcotti W, Zimmermann U \& Knipper M (2013) Otoferlin couples to clathrin-mediated endocytosis in mature cochlear inner hair cells. J. Neurosci. 33: 950819

Eggeling C, Willig KI, Sahl SJ \& Hell SW (2015) Lens-based fluorescence nanoscopy. Q. Rev. Biophys. 48: 178-243

Eltoum I, Fredenburgh J, Myers R \& Grizzle W (2001) Introduction to the theory and practice of fixation of tissues. J Histotechnol 24: 173-190

Eybalin M (1993) Neurotransmitters and neuromodulators of the mammalian cochlea. Physiol. Rev. 73: 309-73

Eybalin M, Renard N, Aure F \& Safieddine S (2002) Cysteine-string protein in inner hair cells of the organ of Corti: synaptic expression and upregulation at the onset of hearing. Eur. J. Neurosci. 15: 1409-20

Faelber K, Held M, Gao S, Posor Y, Haucke V, Noé F \& Daumke O (2012) Structural insights 
into dynamin-mediated membrane fission. Structure 20: 1621-8

Farr AG \& Nakane PK (1981) Immunohistochemistry with enzyme labeled antibodies: a brief review. J. Immunol. Methods 47: 129-44

Fernandes D \& Carvalho AL (2016) Mechanisms of homeostatic plasticity in the excitatory synapse. J. Neurochem. 139: 973-996

Fernández-Chacón R, Achiriloaie M, Janz R, Albanesi JP \& Südhof TC (2000) SCAMP1 function in endocytosis. J. Biol. Chem. 275: 12752-6

Fettiplace R (2017) Hair Cell Transduction, Tuning, and Synaptic Transmission in the Mammalian Cochlea. Compr. Physiol. 7: 1197-1227

Fettiplace R \& Hackney CM (2006) The sensory and motor roles of auditory hair cells. Nat. Rev. Neurosci. 7: 19-29

Fornasiero EF, Mandad S, Wildhagen H, Alevra M, Rammner B, Keihani S, Opazo F, Urban I, Ischebeck T, Sakib MS, Fard MK, Kirli K, Centeno TP, Vidal RO, Rahman R-U, Benito E, Fischer A, Dennerlein S, Rehling P, Feussner I, et al (2018) Precisely measured protein lifetimes in the mouse brain reveal differences across tissues and subcellular fractions. Nat. Commun. 9: 4230

Fornasiero EF \& Opazo F (2015) Super-resolution imaging for cell biologists: concepts, applications, current challenges and developments. Bioessays 37: 436-51

Frank T, Rutherford M a, Strenzke N, Neef A, Pangršič T, Khimich D, Fejtova A, Fetjova A, Gundelfinger ED, Liberman MC, Harke B, Bryan KE, Lee A, Egner A, Riedel D \& Moser T (2010) Bassoon and the synaptic ribbon organize $\mathrm{Ca}^{2}+$ channels and vesicles to add release sites and promote refilling. Neuron 68: 724-38

Fuchs PA \& Glowatzki E (2015) Synaptic studies inform the functional diversity of cochlear afferents. Hear. Res. 330: 18-25

Furness DN \& Hackney CM (2006) The Structure and Composition of the Stereociliary Bundle of Vertebrate Hair Cells. In Eatock RA, Fay RR, Popper AN (eds) Vertebrate Hair Cells pp 95-153. New York: Springer-Verlag

Geppert M, Archer BT \& Südhof TC (1991) Synaptotagmin II. A novel differentially distributed form of synaptotagmin. J. Biol. Chem. 266: 13548-52

Geppert M, Goda Y, Stevens CF \& Südhof TC (1997) The small GTP-binding protein Rab3A regulates a late step in synaptic vesicle fusion. Nature 387: 810-4

Gerber SA, Rush J, Stemman O, Kirschner MW \& Gygi SP (2003) Absolute quantification of proteins and phosphoproteins from cell lysates by tandem MS. Proc. Natl. Acad. Sci. U.

S. A. 100: 6940-5

Gibson DG, Young L, Chuang R, Venter JC, Hutchison CA \& Smith HO (2009) Enzymatic assembly of DNA molecules up to several hundred kilobases. Nat. Methods 6: 343-5

Gil-Loyzaga P \& Pujol R (1988) Synaptophysin in the developing cochlea. Int. J. Dev. Neurosci. 6: $155-60$

Granseth B, Odermatt B, Royle SJ \& Lagnado L (2006) Clathrin-mediated endocytosis is the dominant mechanism of vesicle retrieval at hippocampal synapses. Neuron 51: 773-86

Graydon CW, Cho S, Li G-L, Kachar B \& von Gersdorff H (2011) Sharp Ca ${ }^{2+}$ nanodomains beneath the ribbon promote highly synchronous multivesicular release at hair cell synapses. J. Neurosci. 31: 16637-50

Griesinger CB, Richards CD \& Ashmore JF (2005) Fast vesicle replenishment allows indefatigable signalling at the first auditory synapse. Nature 435: 212-5

Guinan JJ (2010) Cochlear efferent innervation and function. Curr. Opin. Otolaryngol. Head Neck Surg. 18: 447-53

Haass C \& Selkoe DJ (2007) Soluble protein oligomers in neurodegeneration: lessons from 
the Alzheimer's amyloid beta-peptide. Nat. Rev. Mol. Cell Biol. 8: 101-12

Hallermann S, Fejtova A, Schmidt H, Weyhersmüller A, Silver RA, Gundelfinger ED \& Eilers J (2010) Bassoon speeds vesicle reloading at a central excitatory synapse. Neuron 68: 710-23

Hao W, Luo Z, Zheng L, Prasad K \& Lafer EM (1999) AP180 and AP-2 interact directly in a complex that cooperatively assembles clathrin. J. Biol. Chem. 274: 22785-94

Haucke V, Neher E \& Sigrist SJ (2011) Protein scaffolds in the coupling of synaptic exocytosis and endocytosis. Nat. Rev. Neurosci. 12: 127-38

Hell SW \& Wichmann J (1994) Breaking the diffraction resolution limit by stimulated emission: stimulated-emission-depletion fluorescence microscopy. Opt. Lett. 19: 780-2

Hickox AE, Wong ACY, Pak K, Strojny C, Ramirez M, Yates JR, Ryan AF \& Savas JN (2017) Global Analysis of Protein Expression of Inner Ear Hair Cells. J. Neurosci. 37: 1320-1339

Hinshaw JE (2000) Dynamin and its role in membrane fission. Annu. Rev. Cell Dev. Biol. 16: 483-519

Hoopmann P, Punge A, Barysch S V, Westphal V, Bückers J, Opazo F, Bethani I, Lauterbach M a, Hell SW \& Rizzoli SO (2010) Endosomal sorting of readily releasable synaptic vesicles. Proc. Natl. Acad. Sci. U. S. A. 107: 19055-60

Hopwood D (1985) Cell and tissue fixation, 1972-1982. Histochem. J. 17: 389-442

Hua Y, Sinha R, Thiel CS, Schmidt R, Hüve J, Martens H, Hell SW, Egner A \& Klingauf J (2011) A readily retrievable pool of synaptic vesicles. Nat. Neurosci. 14: 833-9

Huang L, Barclay M, Lee K, Peter S, Housley GD, Thorne PR \& Montgomery JM (2012) Synaptic profiles during neurite extension, refinement and retraction in the developing cochlea. Neural Dev. 7: 38

Itakura E, Kishi-Itakura C \& Mizushima N (2012) The hairpin-type tail-anchored SNARE syntaxin 17 targets to autophagosomes for fusion with endosomes/lysosomes. Cell 151: 1256-69

Jackman SL, Choi S-Y, Thoreson WB, Rabl K, Bartoletti TM \& Kramer RH (2009) Role of the synaptic ribbon in transmitting the cone light response. Nat. Neurosci. 12: 303-10

Jahn R \& Fasshauer D (2012) Molecular machines governing exocytosis of synaptic vesicles. Nature 490: 201-7

Jahn R, Schiebler W \& Greengard P (1984) A quantitative dot-immunobinding assay for proteins using nitrocellulose membrane filters. Proc. Natl. Acad. Sci. U. S. A. 81: 1684-7

Jan LY \& Jan YN (1976) Properties of the larval neuromuscular junction in Drosophila melanogaster. J. Physiol. 262: 189-214

Jiménez N, Humbel BM, van Donselaar E, Verkleij AJ \& Burger KNJ (2006) Aclar discs: a versatile substrate for routine high-pressure freezing of mammalian cell monolayers. $J$. Microsc. 221: 216-23

Johnson CP \& Chapman ER (2010) Otoferlin is a calcium sensor that directly regulates SNARE-mediated membrane fusion. J. Cell Biol. 191: 187-97

Johnson SL, Eckrich T, Kuhn S, Zampini V, Franz C, Ranatunga KM, Roberts TP, Masetto S, Knipper M, Kros CJ \& Marcotti W (2011) Position-dependent patterning of spontaneous action potentials in immature cochlear inner hair cells. Nat. Neurosci. 14: 711-7

Jung S, Maritzen T, Wichmann C, Jing Z, Neef A, Revelo NH, Al-Moyed H, Meese S, Wojcik SM, Panou I, Bulut H, Schu P, Ficner R, Reisinger E, Rizzoli SO, Neef J, Strenzke N, Haucke V \& Moser T (2015) Disruption of adaptor protein $2 \mu$ (AP-2 $\mu$ ) in cochlear hair cells impairs vesicle reloading of synaptic release sites and hearing. EMBO J. 34: 2686702

Kaech S \& Banker G (2006) Culturing hippocampal neurons. Nat. Protoc. 1: 2406-15 
Kamin D, Revelo NH \& Rizzoli SO (2014) FM dye photo-oxidation as a tool for monitoring membrane recycling in inner hair cells. PLoS One 9: e88353

Kantardzhieva A, Peppi M, Lane WS \& Sewell WF (2012) Protein composition of immunoprecipitated synaptic ribbons. J. Proteome Res. 11: 1163-74

Kauer JA \& Malenka RC (2007) Synaptic plasticity and addiction. Nat. Rev. Neurosci. 8: 84458

Khimich D, Nouvian R, Pujol R, Tom Dieck S, Egner A, Gundelfinger ED \& Moser T (2005) Hair cell synaptic ribbons are essential for synchronous auditory signalling. Nature 434: 88994

Koo SJ, Markovic S, Puchkov D, Mahrenholz CC, Beceren-Braun F, Maritzen T, Dernedde J, Volkmer R, Oschkinat H \& Haucke V (2011) SNARE motif-mediated sorting of synaptobrevin by the endocytic adaptors clathrin assembly lymphoid myeloid leukemia (CALM) and AP180 at synapses. Proc. Natl. Acad. Sci. U. S. A. 108: 13540-5

Von Kriegstein K, Schmitz F, Link E \& Südhof TC (1999) Distribution of synaptic vesicle proteins in the mammalian retina identifies obligatory and facultative components of ribbon synapses. Eur. J. Neurosci. 11: 1335-48

Kroll J, Jaime Tobón LM, Vogl C, Neef J, Kondratiuk I, König M, Strenzke N, Wichmann C, Milosevic I \& Moser T (2019) Endophilin-A regulates presynaptic Ca2+ influx and synaptic vesicle recycling in auditory hair cells. EMBO J. 38: e100116

Laemmli UK (1970) Cleavage of structural proteins during the assembly of the head of bacteriophage T4. Nature 227: 680-5

Lang T \& Rizzoli SO (2010) Membrane Protein Clusters at Nanoscale Resolution: More Than Pretty Pictures. Physiology 25: 116-124

Latendresse JR, Warbrittion AR, Jonassen H \& Creasy DM (2002) Fixation of testes and eyes using a modified Davidson's fluid: comparison with Bouin's fluid and conventional Davidson's fluid. Toxicol. Pathol. 30: 524-33

Lázaro DF, Rodrigues EF, Langohr R, Shahpasandzadeh H, Ribeiro T, Guerreiro P, Gerhardt E, Kröhnert K, Klucken J, Pereira MD, Popova B, Kruse N, Mollenhauer B, Rizzoli SO, Braus GH, Danzer KM \& Outeiro TF (2014) Systematic comparison of the effects of alphasynuclein mutations on its oligomerization and aggregation. PLoS Genet. 10: e1004741

Lenzi D, Crum J, Ellisman MH \& Roberts WM (2002) Depolarization redistributes synaptic membrane and creates a gradient of vesicles on the synaptic body at a ribbon synapse. Neuron 36: 649-59

Lenzi D \& von Gersdorff H (2001) Structure suggests function: the case for synaptic ribbons as exocytotic nanomachines. Bioessays 23: 831-40

Lenzi D, Runyeon JW, Crum J, Ellisman MH \& Roberts WM (1999) Synaptic vesicle populations in saccular hair cells reconstructed by electron tomography. J. Neurosci. 19: 119-32

Li Y, Liu H, Giffen KP, Chen L, Beisel KW \& He DZZ (2018) Transcriptomes of cochlear inner and outer hair cells from adult mice. Sci. data 5: 180199

Liberman LD, Wang H \& Liberman MC (2011) Opposing gradients of ribbon size and AMPA receptor expression underlie sensitivity differences among cochlear-nerve/hair-cell synapses. J. Neurosci. 31: 801-8

Liberman MC (1980) Morphological differences among radial afferent fibers in the cat cochlea: an electron-microscopic study of serial sections. Hear. Res. 3: 45-63

Liu H, Bai H, Hui E, Yang L, Evans CS, Wang Z, Kwon SE \& Chapman ER (2014a) Synaptotagmin 7 functions as a Ca2+-sensor for synaptic vesicle replenishment. Elife 3: e01524

Liu H, Pecka JL, Zhang Q, Soukup GA, Beisel KW \& He DZZ (2014b) Characterization of 
transcriptomes of cochlear inner and outer hair cells. J. Neurosci. 34: 11085-95

Löschberger A, van de Linde S, Dabauvalle M-C, Rieger B, Heilemann M, Krohne G \& Sauer M (2012) Super-resolution imaging visualizes the eightfold symmetry of gp210 proteins around the nuclear pore complex and resolves the central channel with nanometer resolution. J. Cell Sci. 125: 570-5

Lu P, Vogel C, Wang R, Yao X \& Marcotte EM (2007) Absolute protein expression profiling estimates the relative contributions of transcriptional and translational regulation. Nat. Biotechnol. 25: 117-24

Ma C, Su L, Seven AB, Xu Y \& Rizo J (2013) Reconstitution of the vital functions of Munc18 and Munc13 in neurotransmitter release. Science 339: 421-5

Magupalli VG, Schwarz K, Alpadi K, Natarajan S, Seigel GM \& Schmitz F (2008) Multiple RIBEYE-RIBEYE interactions create a dynamic scaffold for the formation of synaptic ribbons. J. Neurosci. 28: 7954-67

Maidorn M, Rizzoli SO \& Opazo F (2016) Tools and limitations to study the molecular composition of synapses by fluorescence microscopy. Biochem. J. 473: 3385-3399

Mallard F, Tang BL, Galli T, Tenza D, Saint-Pol A, Yue X, Antony C, Hong W, Goud B \& Johannes $L$ (2002) Early/recycling endosomes-to-TGN transport involves two SNARE complexes and a Rab6 isoform. J. Cell Biol. 156: 653-64

Malmström J, Beck M, Schmidt A, Lange V, Deutsch EW \& Aebersold R (2009) Proteomewide cellular protein concentrations of the human pathogen Leptospira interrogans. Nature 460: 762-5

Mandell JW, Townes-Anderson E, Czernik AJ, Cameron R, Greengard P \& De Camilli P (1990) Synapsins in the vertebrate retina: absence from ribbon synapses and heterogeneous distribution among conventional synapses. Neuron 5: 19-33

Mann ZF \& Kelley MW (2011) Development of tonotopy in the auditory periphery. Hear. Res. 276: 2-15

Matern MS, Beirl A, Ogawa Y, Song Y, Paladugu N, Kindt KS \& Hertzano R (2018) Transcriptomic Profiling of Zebrafish Hair Cells Using RiboTag. Front. cell Dev. Biol. 6: 47

Matthews $G$ \& Fuchs P (2010) The diverse roles of ribbon synapses in sensory neurotransmission. Nat. Rev. Neurosci. 11: 812-22

McDonald KL \& Webb RI (2011) Freeze substitution in 3 hours or less. J. Microsc. 243: 22733

McMahon HT \& Boucrot E (2011) Molecular mechanism and physiological functions of clathrin-mediated endocytosis. Nat. Rev. Mol. Cell Biol. 12: 517-33

McMahon HT \& Gallop JL (2005) Membrane curvature and mechanisms of dynamic cell membrane remodelling. Nature 438: 590-6

Melan MA (1994) Overview of cell fixation and permeabilization. Methods Mol. Biol. 34: 5566

Meyer AC, Frank T, Khimich D, Hoch G, Riedel D, Chapochnikov NM, Yarin YM, Harke B, Hell SW, Egner A \& Moser T (2009) Tuning of synapse number, structure and function in the cochlea. Nat. Neurosci. 12: 444-53

Michalski N, Goutman JD, Auclair SM, Boutet de Monvel J, Tertrais M, Emptoz A, Parrin A, Nouaille S, Guillon M, Sachse M, Ciric D, Bahloul A, Hardelin J-P, Sutton RB, Avan P, Krishnakumar SS, Rothman JE, Dulon D, Safieddine S \& Petit C (2017) Otoferlin acts as a $\mathrm{Ca} 2+$ sensor for vesicle fusion and vesicle pool replenishment at auditory hair cell ribbon synapses. Elife 6: 1-34

Michanski S, Smaluch K, Steyer AM, Chakrabarti R, Setz C, Oestreicher D, Fischer C, Möbius W, Moser T, Vogl C \& Wichmann C (2019) Mapping developmental maturation of inner 
hair cell ribbon synapses in the apical mouse cochlea. Proc. Natl. Acad. Sci. U. S. A. 116: 6415-6424

Migneault I, Dartiguenave C, Bertrand MJ \& Waldron KC (2004) Glutaraldehyde: behavior in aqueous solution, reaction with proteins, and application to enzyme crosslinking. Biotechniques 37: 790-6, 798-802

Mim C \& Unger VM (2012) Membrane curvature and its generation by BAR proteins. Trends Biochem. Sci. 37: 526-33

Morelli E, Ginefra P, Mastrodonato V, Beznoussenko G V., Rusten TE, Bilder D, Stenmark H, Mironov AA \& Vaccari T (2014) Multiple functions of the SNARE protein Snap29 in autophagy, endocytic, and exocytic trafficking during epithelial formation in Drosophila. Autophagy 10: 2251-68

Moser T \& Beutner D (2000) Kinetics of exocytosis and endocytosis at the cochlear inner hair cell afferent synapse of the mouse. Proc. Natl. Acad. Sci. U. S. A. 97: 883-8

Moser T, Neef A \& Khimich D (2006) Mechanisms underlying the temporal precision of sound coding at the inner hair cell ribbon synapse. J. Physiol. 576: 55-62

Neef J, Jung S, Wong AB, Reuter K, Pangršič T, Chakrabarti R, Kügler S, Lenz C, Nouvian R, Boumil RM, Frankel WN, Wichmann C \& Moser T (2014) Modes and regulation of endocytic membrane retrieval in mouse auditory hair cells. J. Neurosci. 34: 705-16

Nicholls DG \& Sihra TS (1986) Synaptosomes possess an exocytotic pool of glutamate. Nature 321: 772-3

Nouvian R, Beutner D, Parsons TD \& Moser T (2006) Structure and function of the hair cell ribbon synapse. J. Membr. Biol. 209: 153-65

Nouvian R, Neef J, Bulankina A V, Reisinger E, Pangršič T, Frank T, Sikorra S, Brose N, Binz T \& Moser T (2011) Exocytosis at the hair cell ribbon synapse apparently operates without neuronal SNARE proteins. Nat. Neurosci. 14: 411-3

Opazo F, Levy M, Byrom M, Schäfer C, Geisler C, Groemer TW, Ellington AD \& Rizzoli SO (2012) Aptamers as potential tools for super-resolution microscopy. Nat. Methods 9: 938-9

Opazo F, Punge A, Bückers J, Hoopmann P, Kastrup L, Hell SW \& Rizzoli SO (2010) Limited intermixing of synaptic vesicle components upon vesicle recycling. Traffic 11: 800-12

Paavilainen L, Edvinsson A, Asplund A, Hober S, Kampf C, Pontén F \& Wester K (2010) The impact of tissue fixatives on morphology and antibody-based protein profiling in tissues and cells. J. Histochem. Cytochem. 58: 237-46

Pang ZP, Sun J, Rizo J, Maximov A \& Südhof TC (2006) Genetic analysis of synaptotagmin 2 in spontaneous and Ca2+-triggered neurotransmitter release. EMBO J. 25: 2039-50

Pangršič T, Lasarow L, Reuter K, Takago H, Schwander M, Riedel D, Frank T, Tarantino LM, Bailey JS, Strenzke N, Brose N, Müller U, Reisinger E \& Moser T (2010) Hearing requires otoferlin-dependent efficient replenishment of synaptic vesicles in hair cells. Nat. Neurosci. 13: 869-76

Pangršič T \& Vogl C (2018) Balancing presynaptic release and endocytic membrane retrieval at hair cell ribbon synapses. FEBS Lett. 592: 3633-3650

Platonova E, Winterflood CM, Junemann A, Albrecht D, Faix J \& Ewers H (2015) Singlemolecule microscopy of molecules tagged with GFP or RFP derivatives in mammalian cells using nanobody binders. Methods 88: 89-97

Pozo K \& Goda Y (2010) Unraveling mechanisms of homeostatic synaptic plasticity. Neuron 66: $337-51$

Prasad K \& Lippoldt RE (1988) Molecular characterization of the AP180 coated vesicle assembly protein. Biochemistry 27: 6098-104 
Prekeris R, Klumperman J, Chen YA \& Scheller RH (1998) Syntaxin 13 mediates cycling of plasma membrane proteins via tubulovesicular recycling endosomes. J. Cell Biol. 143: 957-71

Regus-Leidig H, Ott C, Löhner M, Atorf J, Fuchs M, Sedmak T, Kremers J, Fejtová A, Gundelfinger ED \& Brandstätter JH (2013) Identification and immunocytochemical characterization of Piccolino, a novel Piccolo splice variant selectively expressed at sensory ribbon synapses of the eye and ear. PLoS One 8: e70373

Reisinger E, Bresee C, Neef J, Nair R, Reuter K, Bulankina A, Nouvian R, Koch M, Bückers J, Kastrup L, Roux I, Petit C, Hell SW, Brose N, Rhee J-S, Kügler S, Brigande J V \& Moser T (2011) Probing the functional equivalence of otoferlin and synaptotagmin 1 in exocytosis. J. Neurosci. 31: 4886-95

Revelo NH, Kamin D, Truckenbrodt S, Wong AB, Reuter-Jessen K, Reisinger E, Moser T \& Rizzoli SO (2014) A new probe for super-resolution imaging of membranes elucidates trafficking pathways. J. Cell Biol. 205: 591-606

Revelo NH \& Rizzoli SO (2015) Application of STED Microscopy to Cell Biology Questions. Methods Mol. Biol. 1251: 213-30

Richter KN, Revelo NH, Seitz KJ, Helm MS, Sarkar D, Saleeb RS, Este ED, Eberle J, Wagner E, Vogl C, Lazaro DF, Richter F, Coy-vergara J, Coceano G, Boyden ES, Duncan RR, Hell SW, Lauterbach MA, Lehnart SE, Moser T, et al (2018) Glyoxal as an alternative fixative to formaldehyde in immunostaining and super-resolution microscopy. EMBO J. 37: 139159

Rizzoli SO (2014) Synaptic vesicle recycling: steps and principles. EMBO J. 33: 788-822

Rizzoli SO, Bethani I, Zwilling D, Wenzel D, Siddiqui TJ, Brandhorst D \& Jahn R (2006) Evidence for early endosome-like fusion of recently endocytosed synaptic vesicles. Traffic 7: 1163-76

Rizzoli SO \& Betz WJ (2005) Synaptic vesicle pools. Nat. Rev. Neurosci. 6: 57-69

Robertson D \& Paki B (2002) Role of L-type Ca2+ channels in transmitter release from mammalian inner hair cells. II. Single-neuron activity. J. Neurophysiol. 87: 2734-40

Rodriguez-Contreras A \& Yamoah EN (2001) Direct measurement of single-channel Ca(2+) currents in bullfrog hair cells reveals two distinct channel subtypes. J. Physiol. 534: 66989

Roux I, Safieddine S, Nouvian R, Grati M, Simmler M-C, Bahloul A, Perfettini I, Le Gall M, Rostaing P, Hamard G, Triller A, Avan P, Moser T \& Petit C (2006) Otoferlin, defective in a human deafness form, is essential for exocytosis at the auditory ribbon synapse. Cell 127: $277-89$

Rubel EW (1984) Ontogeny of auditory system function. Annu. Rev. Physiol. 46: 213-29

Ruel J, Emery S, Nouvian R, Bersot T, Amilhon B, Van Rybroek JM, Rebillard G, Lenoir M, Eybalin M, Delprat B, Sivakumaran TA, Giros B, El Mestikawy S, Moser T, Smith RJH, Lesperance MM \& Puel J-L (2008) Impairment of SLC17A8 encoding vesicular glutamate transporter-3, VGLUT3, underlies nonsyndromic deafness DFNA25 and inner hair cell dysfunction in null mice. Am. J. Hum. Genet. 83: 278-92

Rutherford M a \& Pangršič T (2012) Molecular anatomy and physiology of exocytosis in sensory hair cells. Cell Calcium 52: 327-37

Rutherford MA (2015) Resolving the structure of inner ear ribbon synapses with STED microscopy. Synapse 69: 242-55

Sabatini DD, Bensch K \& Barrnett RJ (1963) Cytochemistry and electron microscopy. The preservation of cellular ultrastructure and enzymatic activity by aldehyde fixation. J. Cell Biol. 17: 19-58 
Safieddine S, El-Amraoui A \& Petit C (2012) The auditory hair cell ribbon synapse: from assembly to function. Annu. Rev. Neurosci. 35: 509-28

Safieddine S \& Wenthold RJ (1997) The glutamate receptor subunit delta1 is highly expressed in hair cells of the auditory and vestibular systems. J. Neurosci. 17: 7523-31

Safieddine S \& Wenthold RJ (1999) SNARE complex at the ribbon synapses of cochlear hair cells: analysis of synaptic vesicle- and synaptic membrane-associated proteins. Eur. J. Neurosci. 11: 803-12

Saito K (1980) Fine structure of the sensory epithelium of the guinea pig organ of Corti: afferent and efferent synapses of hair cells. J. Ultrastruct. Res. 71: 222-32

Scheffer DI, Shen J, Corey DP \& Chen Z-Y (2015) Gene Expression by Mouse Inner Ear Hair Cells during Development. J. Neurosci. 35: 6366-80

Schlüter OM, Basu J, Südhof TC \& Rosenmund C (2006) Rab3 superprimes synaptic vesicles for release: implications for short-term synaptic plasticity. J. Neurosci. 26: 1239-46

Schlüter OM, Schmitz F, Jahn R, Rosenmund C \& Südhof TC (2004) A complete genetic analysis of neuronal Rab3 function. J. Neurosci. 24: 6629-37

Schmitz F (2009) The making of synaptic ribbons: how they are built and what they do. Neuroscientist 15: 611-24

Schmitz F, Königstorfer A \& Südhof TC (2000) RIBEYE, a component of synaptic ribbons: a protein's journey through evolution provides insight into synaptic ribbon function. Neuron 28: 857-72

Schmitz F, Tabares L, Khimich D, Strenzke N, de la Villa-Polo P, Castellano-Muñoz M, Bulankina A, Moser T, Fernández-Chacón R \& Südhof TC (2006) CSPalpha-deficiency causes massive and rapid photoreceptor degeneration. Proc. Natl. Acad. Sci. U. S. A. 103: 2926-31

Schnee ME, Santos-Sacchi J, Castellano-Muñoz M, Kong J-H \& Ricci AJ (2011) Calciumdependent synaptic vesicle trafficking underlies indefatigable release at the hair cell afferent fiber synapse. Neuron 70: 326-38

Schnell U, Dijk F, Sjollema KA \& Giepmans BNG (2012) Immunolabeling artifacts and the need for live-cell imaging. Nat. Methods 9: 152-8

Schwanhäusser B, Busse D, Li N, Dittmar G, Schuchhardt J, Wolf J, Chen W \& Selbach M (2011) Global quantification of mammalian gene expression control. Nature 473: 337342

Seal RP, Akil O, Yi E, Weber CM, Grant L, Yoo J, Clause A, Kandler K, Noebels JL, Glowatzki E, Lustig LR \& Edwards RH (2008) Sensorineural deafness and seizures in mice lacking vesicular glutamate transporter 3. Neuron 57: 263-75

Sharma M, Burré J \& Südhof TC (2011) CSP $\alpha$ promotes SNARE-complex assembly by chaperoning SNAP-25 during synaptic activity. Nat. Cell Biol. 13: 30-9

Shnyrova A V, Bashkirov P V, Akimov SA, Pucadyil TJ, Zimmerberg J, Schmid SL \& Frolov VA (2013) Geometric catalysis of membrane fission driven by flexible dynamin rings. Science 339: 1433-6

Siegel JH \& Brownell WE (1986) Synaptic and Golgi membrane recycling in cochlear hair cells. J. Neurocytol. 15: 311-28

Simonsen A, Gaullier JM, D'Arrigo A \& Stenmark H (1999) The Rab5 effector EEA1 interacts directly with syntaxin-6. J. Biol. Chem. 274: 28857-60

Slepnev VI \& De Camilli P (2000) Accessory factors in clathrin-dependent synaptic vesicle endocytosis. Nat. Rev. Neurosci. 1: 161-72

Smith CA \& Sjöstrand FS (1961) A synaptic structure in the hair cells of the guinea pig cochlea. J. Ultrastruct. Res. 5: 184-192 
Smith JE \& Reese TS (1980) Use of aldehyde fixatives to determine the rate of synaptic transmitter release. J. Exp. Biol. 89: 19-29

Snellman J, Mehta B, Babai N, Bartoletti TM, Akmentin W, Francis A, Matthews G, Thoreson W \& Zenisek D (2011) Acute destruction of the synaptic ribbon reveals a role for the ribbon in vesicle priming. Nat. Neurosci. 14: 1135-41

Sobkowicz HM, Rose JE, Scott GL \& Levenick C V. (1986) Distribution of synaptic ribbons in the developing organ of Corti. J. Neurocytol. 15: 693-714

Steegmaier M, Klumperman J, Foletti DL, Yoo JS \& Scheller RH (1999) Vesicle-associated membrane protein 4 is implicated in trans-Golgi network vesicle trafficking. Mol. Biol. Cell 10: 1957-72

Steegmaier M, Yang B, Yoo JS, Huang B, Shen M, Yu S, Luo Y \& Scheller RH (1998) Three novel proteins of the syntaxin/SNAP-25 family. J. Biol. Chem. 273: 34171-9

Stevens RJ, Akbergenova Y, Jorquera RA \& Littleton JT (2012) Abnormal synaptic vesicle biogenesis in Drosophila synaptogyrin mutants. J. Neurosci. 32: 18054-67, 18067a

Südhof TC (2004) The synaptic vesicle cycle. Annu. Rev. Neurosci. 27: 509-47

Südhof TC (1995) The synaptic vesicle cycle: a cascade of protein-protein interactions. Nature 375: 645-53

Südhof TC (2008) Neuroligins and neurexins link synaptic function to cognitive disease. Nature 455: 903-11

Sugita S, Han W, Butz S, Liu X, Fernández-Chacón R, Lao Y \& Südhof TC (2001) Synaptotagmin VII as a plasma membrane $\mathrm{Ca}(2+)$ sensor in exocytosis. Neuron 30: 459-73

Sugiyama Y, Kawabata I, Sobue K \& Okabe S (2005) Determination of absolute protein numbers in single synapses by a GFP-based calibration technique. Nat. Methods 2: 67784

Swaab DF, Pool CW \& Nijveldt F (1975) Immunofluorescence of vasopressin and oxytocin in the rat hypothalamo-neurohypophypopseal system. J. Neural Transm. 36: 195-215

Takago H, Oshima-Takago T \& Moser T (2018) Disruption of Otoferlin Alters the Mode of Exocytosis at the Mouse Inner Hair Cell Ribbon Synapse. Front. Mol. Neurosci. 11: 492

Takamori S, Holt M, Stenius K, Lemke EA, Grønborg M, Riedel D, Urlaub H, Schenck S, Brügger B, Ringler P, Müller SA, Rammner B, Gräter F, Hub JS, De Groot BL, Mieskes G, Moriyama Y, Klingauf J, Grubmüller H, Heuser J, et al (2006) Molecular anatomy of a trafficking organelle. Cell 127: 831-46

Tanaka K a K, Suzuki KGN, Shirai YM, Shibutani ST, Miyahara MSH, Tsuboi H, Yahara M, Yoshimura A, Mayor S, Fujiwara TK \& Kusumi A (2010) Membrane molecules mobile even after chemical fixation. Nat. Methods 7: 865-6

Tang BL, Low DYH, Lee SS, Tan AEH \& Hong W (1998) Molecular cloning and localization of human syntaxin 16, a member of the syntaxin family of SNARE proteins. Biochem. Biophys. Res. Commun. 242: 673-9

Titford ME \& Horenstein MG (2005) Histomorphologic assessment of formalin substitute fixatives for diagnostic surgical pathology. Arch. Pathol. Lab. Med. 129: 502-6

Trapani JG, Obholzer N, Mo W, Brockerhoff SE \& Nicolson T (2009) Synaptojanin1 is required for temporal fidelity of synaptic transmission in hair cells. PLoS Genet. 5: e1000480

Truckenbrodt S, Maidorn M, Crzan D, Wildhagen H, Kabatas S \& Rizzoli SO (2018a) X10 expansion microscopy enables $25-\mathrm{nm}$ resolution on conventional microscopes. $E M B O$ Rep.: e45836

Truckenbrodt S, Viplav A, Jähne S, Vogts A, Denker A, Wildhagen H, Fornasiero EF \& Rizzoli SO (2018b) Newly produced synaptic vesicle proteins are preferentially used in synaptic transmission. EMBO J. 37: 
Umlas J \& Tulecke M (2004) The effects of glyoxal fixation on the histological evaluation of breast specimens. Hum. Pathol. 35: 1058-62

Uthaiah RC \& Hudspeth a J (2010) Molecular anatomy of the hair cell's ribbon synapse. J. Neurosci. 30: 12387-99

Vreja IC, Nikić I, Göttfert F, Bates M, Kröhnert K, Outeiro TF, Hell SW, Lemke EA \& Rizzoli SO (2015) Super-resolution Microscopy of Clickable Amino Acids Reveals the Effects of Fluorescent Protein Tagging on Protein Assemblies. ACS Nano 9: 11034-41]

Wagner E, Brandenburg S, KohI T \& Lehnart SE (2014) Analysis of Tubular Membrane Networks in Cardiac Myocytes from Atria and Ventricles. J Vis Exp 92

Wagner E, Lauterbach MA, KohI T, Westphal V, Williams GSB, Steinbrecher JH, Streich JH, Korff B, Tuan HT, Hagen B, Luther S, Hasenfuss G, Parlitz U, Jafri MS, Hell SW, Lederer WJ, Lehnart SE (2012) Stimulated Emission Depletion Live-Cell Super-Resolution Imaging Shows Proliferative Remodeling of T-Tubule Membrane Structures After Myocardial Infarction. Circ. Res.111: 402-14

Wahl S, Katiyar R \& Schmitz F (2013) A local, periactive zone endocytic machinery at photoreceptor synapses in close vicinity to synaptic ribbons. J. Neurosci. 33: 10278-300

Wangemann $P$ (2006) Supporting sensory transduction: cochlear fluid homeostasis and the endocochlear potential. J. Physiol. 576: 11-21

Wenthold RJ, Safieddine S, Ly CD, Wang Y-X, Lee H-K, Wang C-Y, Kachar B \& Petralia RS (2002) Vesicle targeting in hair cells. Audiol. Neurootol. 7: 45-8

Wichmann C \& Moser T (2015) Relating structure and function of inner hair cell ribbon synapses. Cell Tissue Res. 361: 95-114

Wicks LF \& Suntzeff V (1943) Glyoxal, a non-irritating aldehyde suggested as substitute for formalin in histological fixations. Science 98: 204

Wienisch M \& Klingauf J (2006) Vesicular proteins exocytosed and subsequently retrieved by compensatory endocytosis are nonidentical. Nat. Neurosci. 9: 1019-27

Wilhelm BG, Mandad S, Truckenbrodt S, Kröhnert K, Schäfer C, Rammner B, Koo SJ, Claßen GA, Krauss M, Haucke V, Urlaub H \& Rizzoli SO (2014) Composition of isolated synaptic boutons reveals the amounts of vesicle trafficking proteins. Science 344: 1023-8

Willig KI, Rizzoli SO, Westphal V, Jahn R \& Hell SW (2006) STED microscopy reveals that synaptotagmin remains clustered after synaptic vesicle exocytosis. Nature 440: 935-9

Wong AB, Rutherford MA, Gabrielaitis M, Pangršič T, Göttfert F, Frank T, Michanski S, Hell S, Wolf F, Wichmann C \& Moser T (2014) Developmental refinement of hair cell synapses tightens the coupling of $\mathrm{Ca} 2+$ influx to exocytosis. EMBO J. 33: 247-64

Wong SH, Xu Y, Zhang T, Griffiths G, Lowe SL, Subramaniam VN, Seow KT \& Hong W (1999) GS32, a novel Golgi SNARE of $32 \mathrm{kDa}$, interacts preferentially with syntaxin 6. Mol. Biol. Cell 10: 119-34

Woodruff JJ \& Rasmussen RA (1979) In vitro adherence of lymphocytes to unfixed and fixed high endothelial cells of lymph nodes. J. Immunol. 123: 2369-72

Xu K, Zhong G \& Zhuang X (2013) Actin, spectrin, and associated proteins form a periodic cytoskeletal structure in axons. Science 339: 452-6

Xue L, Karpenko IA, Hiblot J \& Johnsson K (2015) Imaging and manipulating proteins in live cells through covalent labeling. Nat. Chem. Biol. 11: 917-23

Yasunaga S, Grati M, Chardenoux S, Smith TN, Friedman TB, Lalwani AK, Wilcox ER \& Petit C (2000) OTOF encodes multiple long and short isoforms: genetic evidence that the long ones underlie recessive deafness DFNB9. Am. J. Hum. Genet. 67: 591-600

Zhang SY, Robertson D, Yates G \& Everett A (1999) Role of L-type Ca(2+) channels in transmitter release from mammalian inner hair cells I. Gross sound-evoked potentials. J. 
Neurophysiol. 82: 3307-15 


\section{8 | List of Abbreviations}

$\begin{array}{ll}\text { a.u. } & \text { arbitrary unit } \\ \text { BD } & \text { BD Biosciences } \\ \text { BSA } & \text { bovine serum albumin } \\ \text { Clathrin LC } & \text { clathrin light chain } \\ \text { Clathrin HC } & \text { clathrin heavy chain } \\ \text { CosiQuant } & \text { comparative synaptosomes imaging for semi-quantitative copy numbers } \\ \text { DMEM } & \text { Dulbecco's Modified Eagle Medium } \\ \text { EM } & \text { electron microscopy } \\ \text { FISH } & \text { fluorescence in-situ hybridization } \\ \text { FWHM } & \text { full width half maximum } \\ \text { GFP } & \text { green fluorescent protein } \\ \text { HBSS } & \text { Hank's balanced salt solution } \\ \text { HEPES } & \text { 4-(2-Hydroxyethyl) piperazine-1-ethanesulfonic acid } \\ \text { IHC } & \text { inner hair cell } \\ \text { MET } & \text { mechanoelectrical transduction } \\ \text { MS } & \text { mass spectrometry } \\ \text { NA } & \text { numerical aperature } \\ \text { OHC } & \text { outer hair cell } \\ \text { PBS } & \text { phosphate-buffered saline } \\ \text { PFA } & \text { paraformaldehyde } \\ \text { PI } & \text { propidium iodide } \\ \text { PIP } & \text { phosphatidylinositol-4,5-bisphosphat } \\ \text { POI } & \text { protein of interest } \\ \text { ROI } & \text { region of interest } \\ \text { RRP } & \text { readily releasable pool } \\ \text { RT } & \text { room temperature } \\ \text { SDS PAGE } & \text { sodiumdodecylsulfate polyacryl gel electrophoresis } \\ \text { SEM } & \text { standard error of the mean } \\ \text { SNARE } & \text { soluble NSF attachment protein receptor } \\ \text { STED } & \text { stimulated emission depletion } \\ \text { SV } & \text { synaptic vesicle } \\ \text { Syn } & \text { synaptosome(s) } \\ \text { SySy } & \text { Synaptic Systems } \\ & \end{array}$




\section{9|Acknowledgements}

With the completion of my PhD thesis I am starting to realize how far I have come within the past 10 years and I know I owe deep gratitude to a number of people, who have contributed to the path that has led me here.

First of all, I would like to thank Prof. Dr. Silvio Rizzoli for providing the opportunity to accomplish the biggest goal of my academic life so far, the completion of a PhD thesis. I am deeply thankful for his excellent supervision during the entire time of my project. Despite the busy schedule, that comes with being a professor, he would always make time to discuss and solve problems with me. His dedication to science is inspiring and taught me a lot beyond the scientific knowledge, which is usually acquired during a PhD. I am also thankful for all the Matlab scripts he provided for analyzing my data and for always pushing me to accomplish more than I thought I could.

I would also like to thank the members of my thesis advisory committee, Prof. Dr. Tomas Pieler and Prof. Dr. Carolin Wichmann, who both provided valuable input for my projects during the thesis advisory meetings. Additionally, I would like to thank Prof. Wichmann for contributing to my IHC ribbon synapse model with the electron microscopy data of the ribbon.

Further, I am grateful for having done my PhD within the CMPB program of the GGNB, who creates optimal conditions for $\mathrm{PhD}$ students to improve their skills and knowledge inside and outside of academia. Thank you to the whole GGNB office for working so hard on maintaining and improving the PhD programs in Göttingen. As PhD representative I was able to get an impression of all the work you do behind the scenes and I really don't want to take that for granted. Of course, being part of the CMPB program wouldn't have been such a pleasure without my fellow PhD students from the program. I very much enjoyed the great times during retreats, seminars and the annual meetings at the Christmas market. Especially important to me was the time spent with Martin, Ulli and Kalle (and of course Nathalie) outside of the scientific agenda. I am also grateful for all the help with the organization of Encephalon, provided by the students of the CMPB.

Everyone who has done his/her PhD or who has witnessed someone doing so, knows that this time can be hard and exhausting. That is why I am truly grateful for having been able to work in a lab that provides such an enjoyable and friendly atmosphere. I want to thank all of my colleagues for creating an environment, which I always enjoyed working in, even in times of frustration and doubt (and let's face it: that is a big part of the time as a PhD student). Most of my colleagues have become so much more than just that and made the past years a truly amazing experience and one of the best times in my life. A big part of my gratitude goes to Kim, Manuel, Sebastian, Sinem, Martin and Eugenio, with who I share memorable moments from times in the lab as well as outside. Kim and Manuel, thank you for many, sometimes more and sometimes less meaningful conversations and in general for being such good friends. I am deeply thankful for Sinems ability to always sense when I needed a hug and for sharing the love for yoga and sushi with me. Thank you Seb for being the best office partner I could have wished for and together with Martin for such amazing times spent at 
conferences around the world. I also want to thank Martin for always providing valuable scientific input when needed and for very pleasant collaborations on multiple publications. I further want to thank Eugenio for always very selflessly providing help when needed, for good bouldering sessions and for even better Italian food. I also owe a lot of gratitude to Natalia, who taught me everything about the dissection and handling of the Organ of Corti and who invested so much work in the glyoxal project.

Of course I am also thankful for the support from people outside my lab and PhD program. I am grateful for every friend who believed in me and supported me along the way. Especially Xzander deserves a great deal of my gratitude for always understanding and for giving me strength when I needed it. Thank you for never getting tired of listening to my problems and showing me that I am not alone.

Probably the biggest gratitude I owe to Verena. I am thankful for your persisting support through good times as well as bad times, for believing in me when I didn't, for celebrating victories and life in general and for inspiring me to be a better person. Sometimes, staying with me might feel like a fight, but I am thankful that you deem this a fight worth fighting. I know I don't even come close to saying this enough, but I am deeply grateful for our relationship.

Lastly, I would like to thank my family, who always believed in me. A great part of my thankfulness goes to my parents, who always made me feel like I could achieve everything. Mama und Papa; ohne euch wäre das alles nicht möglich gewesen. Danke, für eure Unterstützung auf meinem gesamten Weg und dass ihr mir immer die Freiheit und Sicherheit gegeben habt, die ich brauchte, um meine eigenen Entscheidungen zu treffen und meine Träume zu vefolgen. 